\title{
Updated S3-Guideline Ulcerative Colitis. German Society for Digestive and Metabolic Diseases (DGVS)
}

AWMF Registry 021/009

\section{Aktualisierte S3-Leitlinie Colitis ulcerosa der Deutschen Gesellschaft für Gastroenterologie, Verdauungs- und Stoffwechselkrankheiten (DGVS)}

AWMF-Register-Nr. 021/009

Authors

Torsten Kucharzik $^{1 *}$, Axel U. Dignass ${ }^{2 *}$, Raja Atreya ${ }^{3}$, Bernd Bokemeyer ${ }^{4}$, Philip Esters ${ }^{2}$, Klaus Herrlinger ${ }^{5}$,

Klaus Kannengießer ${ }^{1}$, Peter Kienle ${ }^{6}$, Jost Langhorst ${ }^{7}$, Andreas Lügering ${ }^{8}$, Stefan Schreiber ${ }^{9}$, Andreas Stallmach ${ }^{10}$, Jürgen Stein ${ }^{11}$, Andreas Sturm ${ }^{12}$, Niels Teich ${ }^{13}$, Britta Siegmund ${ }^{14}$

Collaborators:

T. Andus, F. Autschbach, O. Bachmann, G. Baretton, D. C. Baumgart, D. Bettenworth, M. Bläker, S. Buderus, J. Büning,

R. Ehehalt, K. Fellermann, S. Fichtner-Feigl, M. Götz, C. Gross, F. Hartmann, P. Hartmann, S. In der Smitten, W. Häuser,

U. Helwig, B. Kaltz, I. Kanbach, K. M. Keller, J. Klaus, S. Koletzko, A. Kroesen, W. Kruis, T. Kühbacher, L. Leifeld, C. Maaser,

H. Matthes, G. Moog, J. Ockenga, A. Pace, M. Reinshagen, E. Rijcken, G. Rogler, E. Stange, C. Veltkamp, J. Zemke

Affiliations

1 Klinik für Allgemeine Innere Medizin und Gastroenterologie, Klinikum Lüneburg, Lüneburg, Deutschland

2 Medizinische Klinik I, Agaplesion Markus Krankenhaus, Frankfurt am Main, Deutschland

3 Medizinische Klinik 1, Universitätsklinikum Erlangen, Deutschland

4 Gastroenterologische Gemeinschaftspraxis Minden, Deutschland

5 Innere Medizin I, Asklepios Klinik Nord Hamburg, Deutschland

6 Allgemein- und Viszeralchirurgie, Theresienkrankenhaus und Sankt Hedwig-Klinik GmbH, Mannheim, Deutschland

7 Innere Medizin V, Naturheilkunde und Integrative Medizin, Kliniken Essen-Mitte, University of Duisburg-Essen, Deutschland

8 Medizinisches Versorgungszentrum Portal 10, Münster, Deutschland

9 Klinik für Innere Medizin I, Kiel, Deutschland

10 Gastroenterologie, Hepatologie und Infektiologie, Friedrich Schiller Universität, Jena, Deutschland

11 Interdisziplinäres Crohn und Colitis Centrum Rhein-Main, Frankfurt/Main, Deutschland

12 Klinik für Innere Medizin mit Schwerpunkt Gastroenterologie, DRK Kliniken Berlin Westend, Berlin, Deutschland

\section{Internistische Gemeinschaftspraxis für Verdauungs- und Stoffwechselkrankheiten, Leipzig, Deutschland \\ 14 Medizinische Klinik I, Charité Universitätsmedizin Berlin, Campus Benjamin Franklin, Berlin, Deutschland}

Key words ulcerative colitis, IBD, diagnosis, treatment, remission, maintenance

\section{Schlüsselwörter}

Colitis ulcerosa, Chronisch-entzündliche Darmerkrankung, Diagnose, Therapie, Remission, Remissionserhaltung

Bibliography

DOI https://doi.org/10.1055/a-0824-0861

Published online: January 17, 2019

Z Gastroenterol 2019; 57: 162-241

(c) Georg Thieme Verlag KG, Stuttgart · New York

ISSN 0044-2771

Correspondence

Prof. Dr. med. Torsten Kucharzik

Klinik für Allgemeine Innere Medizin und Gastroenterologie, Städtisches Klinikum Lüneburg, Bögelstr. 1, 21339 Lüneburg torsten.kucharzik@klinikum-lueneburg.de

Prof. Dr. med. Axel Dignass

Medizinische Klinik I, Agaplesion Markus Krankenhaus,

Wilhelm-Epstein-Str. 4, 60431 Frankfurt

axel.dignass@fdk.info 


\section{ZUSAMMENFASSUNG}

Die neue S3-Leitlinie Colitis stellt aktuelle und evidenzbasierte Empfehlungen zur Behandlung der Colitis ulcerosa zur Verfügung. Sie ersetzt damit die Vorläuferversion von 2011. Neben den neuesten Erkenntnissen zu Diagnostik und Therapie werden insbesondere infektiologische Probleme, chirurgische und Ernährungsmaßnahmen aufgegriffen. Unter der Federführung der DGVS wurde die Leitlinie gemeinsam mit 10 weiteren Fachgesellschaften und Patientenvertretern erarbeitet mit dem Ziel, eine optimale interdisziplinäre Versorgung der Patienten zu gewährleisten.

\section{ABSTRACT}

This guideline provides evidence-based key recommendations for diagnosis and treatment of ulcerative colitis and upgrades the 2011 version. The guideline was developed by an interdisciplinary team of gastroenterologists, surgeons, pathologists, nutrition experts, and patient support groups under the auspice of the German Society for Gastroenterology and Metabolic Diseases. The guideline used structural S3 consensus-based methodology and includes statements on clinical practice, prevention, infectiological problems, surgery and nutrition.

\begin{tabular}{|c|c|c|}
\hline \multicolumn{2}{|c|}{ Contents } & \multirow{2}{*}{$\begin{array}{c}\text { Page } \\
163\end{array}$} \\
\hline 1. & About these guidelines & \\
\hline & Publisher & 163 \\
\hline & Methodological precision & 164 \\
\hline & External evaluation and approval & 166 \\
\hline & Distribution and implementation & 166 \\
\hline & Editorial note & 168 \\
\hline & Special note & 168 \\
\hline \multirow[t]{4}{*}{2.} & Diagnostics & 168 \\
\hline & Classification & 168 \\
\hline & Medical History & 169 \\
\hline & Diagnosis & 170 \\
\hline \multirow[t]{8}{*}{3.} & $\begin{array}{l}\text { Treatment of active disease and remission } \\
\text { maintenance therapy }\end{array}$ & 180 \\
\hline & General therapeutic goals & 180 \\
\hline & Therapy of uncomplicated ulcerative colitis & 181 \\
\hline & $\begin{array}{l}\text { Remission maintenance in primarily uncomplicated } \\
\text { ulcerative colitis }\end{array}$ & 182 \\
\hline & $\begin{array}{l}\text { Complicated forms of disease progression in } \\
\text { ulcerative colitis/severe ulcerative colitis }\end{array}$ & 185 \\
\hline & Steroid-dependent ulcerative colitis & 189 \\
\hline & $\begin{array}{l}\text { The use of biosimilars in patients with ulcerative } \\
\text { colitis }\end{array}$ & 192 \\
\hline & $\begin{array}{l}\text { Maintenance therapy of remission in patients with } \\
\text { complicated disease progression in ulcerative colitis }\end{array}$ & 193 \\
\hline 4. & Infectious problems & 197 \\
\hline 5. & Surgery & 203 \\
\hline 5.1 & Surgical Techniques & 203 \\
\hline 5.2 & Pouchitis & 210 \\
\hline 6. & Nutrition and complementary therapies & 212 \\
\hline 6.1 & $\begin{array}{l}\text { Nutrition in the aetiology and prevention of } \\
\text { ulcerative colitis }\end{array}$ & 212 \\
\hline 6.2 & Complementary therapies & 215 \\
\hline \multicolumn{2}{|c|}{ Abbreviations } & 219 \\
\hline \multicolumn{2}{|c|}{ References } & 220 \\
\hline
\end{tabular}

\section{About these guidelines}

\section{Publisher}

\section{Coordinating professional association}

German Society for Gastroenterology, Digestive and Metabolic Diseases (Deutsche Gesellschaft für Gastroenterologie, Verdauungs- und Stoffwechselerkrankungen, DGVS).

\section{Scope and goals}

In Germany, about 150000 people suffer from ulcerative colitis. For most patients, the disease begins during school or vocational training and continues throughout their lives. Ulcerative colitis not only causes individual suffering and a reduced quality of life; it also causes considerable costs for society. However, many patients still do not receive adequate therapy. The updating of the guidelines, which were last updated in 2011, is therefore considered particularly important by the professional associations involved.

\section{Aims and objectives}

The aim of the guideline is to be easy to apply in general practice, internal medicine, surgery, paediatrics and gastroenterology. Cases of especially severe and/or complicated disease, as encountered in specialised treatment centres or outpatient units, may exceed the scope of this guideline.

Since the subtopics "Extraintestinal manifestations", "IBDassociated diseases" and "Pain" were dealt with in the last Crohn's Disease guidelines of 2014, these sections have not been reviewed in the present ulcerative colitis guideline. "Infectious problems" as well as the topic "Nutrition" were not specifically addressed in the last Crohn's disease guidelines, and were therefore specifically dealt with in this guideline.

Patient target groups are patients with ulcerative colitis of any age.

\section{Target user group}

This guideline is designed for use by all healthcare professionals involved in the diagnostics and therapy of patients with ulcerative colitis, including general practitioners, paediatricians, pathologists, consultant gastroenterologists and specialists in internal medicine, surgeons, IBD nurses and assistants, as well as patients, 
- Table 1 Steering committee.

\begin{tabular}{|l|l|l|}
\hline name & location & responsibilities \\
\hline B. Bokemeyer & Minden & consultant gastroenterologists' representative, Competence Network IBD (Kompetenznetz KN-CED) \\
\hline A. Dignass & Frankfurt & $\begin{array}{l}\text { coordinator, Working Group of Leading Hospital Gastroenterologists (Arbeitsgemeinschaft leitender gastroentero- } \\
\text { logischer Krankenhausärzte, ALGK), German Society for Gastroenterology, Digestive and Metabolic Diseases (DGVS) }\end{array}$ \\
\hline B. Kaltz & Berlin & German Crohn's Disease/Ulcerative Colitis Association (Deutsche Morbus Crohn/Colitis ulcerosa Vereinigung, DCCV) \\
\hline T. Kucharzik & Lüneburg & coordinator, ALGK, European Crohn's and Colitis Organization (ECCO) \\
\hline S. Schreiber & Kiel & Competence Network IBD, university-based gastroenterology \\
\hline B. Siegmund & Berlin & ECCO, university-based gastroenterology, DGVS \\
\hline
\end{tabular}

relatives and healthcare providers (health insurance funds and pension insurance institutions).

Constitution of the guideline group: Involvement of professional societies, specialist networks and patient associations

The revision of the guidelines was led by two main coordinators (Axel Dignass, Frankfurt and Torsten Kucharzik, Lüneburg) in close coordination with a steering group ( $\vee$ Table 1 ).

Alongside the steering committee, five working groups (WGs) were formed, each headed by two group leaders ( $\triangleright$ Table 2 ). Due to the considerable extent of the topics to be covered, the WG on diagnostics was headed by three group leaders. Each WG comprised balanced proportions of university-based and non-university-based physicians, hospital physicians and office-based physicians. Along with gastroenterologists and surgeons, the WGs included other healthcare professionals including paediatricians, pathologists, specialists in complementary medicine, clinical nutrition experts, specialist IBD nurses and assistants (Society of Medical Assistants for IBD (FACED)), as well as patients with IBD (DCCV).

All members of each WG participated in an online survey, and almost all took part in the consensus conference (CC).

Representativity of the guideline team: Participating professional societies

- DGVS; German Society for Gastroenterology, Digestive and Metabolic Diseases (Deutsche Gesellschaft für Gastroenterologie, Verdauungs- und Stoffwechselkrankheiten)

- DGAV; German Society for General and Visceral Surgery (Deutsche Gesellschaft für Allgemein- und Viszeralchirurgie)

- DGCH; German Society of Surgery (Deutsche Gesellschaft für Chirurgie)

- GPGE; Society of Paediatric Gastroenterology and Nutrition (Gesellschaft für pädiatrische Gastroenterologie und Ernährungsmedizin)

- KN-CED; Competence Network IBD (Kompetenznetz Darmerkrankungen)

- DCCV e. V.; German Crohn's Disease/Ulcerative Colitis Association (Deutsche Morbus Crohn/Colitis ulcerosa Vereinigung)

- FACED; Society of Medical Assistants for Inflammatory Bowel Disease (Fachangestellte für chronisch entzündliche Darmerkrankungen)
- DGEM; German Nutrition Society (Deutsche Gesellschaft für Ernährungsmedizin)

- DGP; Germany Society of Pathology (Deutsche Gesellschaft für Pathologie)

- DGK; German Society of Coloproctology (Deutsche Gesellschaft für Koloproktologie)

Also invited to participate in the guideline revision were the German Society of General Medicine and Family Medicine (Deutsche Gesellschaft für Allgemeinmedizin und Familienmedizin, DEGAM), which was unable to join the guidelines team due to insufficient availability of personnel, and the German Society of Internal Medicine (Deutsche Gesellschaft für Innere Medizin e. V. DGIM), which was also unable to join.

\section{Representativity of the guideline team: Direct patient} participation

Several patient members of the German Crohn's Disease/Ulcerative Colitis Association (Deutsche Morbus Crohn/Colitis ulcerosa Vereinigung (DCCV) e. V.) were directly involved as members of the working groups.

\section{Methodological Precision}

Research strategies, choice and evaluation of scientific evidence (evidence base)

The previous version of this guideline was the $\$ 3$ Guideline for Ulcerative Colitis of 2011 and 2008. Due to new methodological requirements, the methodology to be applied was discussed in a telephone conference within the steering group on July 5, 2016 and subsequently agreed upon before the start of the revision.

The search strategy of the last colitis guideline was revised and edited by the coordinators together with the working group leaders. The clinical guideline services usergroup (CGS) was commissioned with the systematic literature search of this update; the literature search was carried out by Maria Kallenbach. Initially, a systematic search was performed for existing guidelines. These were evaluated using the German Instrument for Methodical Guideline Evaluation (Deutsche Leitlinien-Bewertungsinstrument, DELBI), and a guideline synopsis was compiled.

For topics of particular clinical importance or controversiality, or subject to frequent misapplication of guidelines, the WG leaders 
- Table 2 Members of the guidelines team.

\begin{tabular}{|c|c|c|}
\hline \multirow[t]{2}{*}{ WG 1: diagnostics } & group leaders & $\begin{array}{l}\text { R. Atreya, Erlangen (DGVS) } \\
\text { B. Bokemeyer, Minden (KN-CED, DGVS) } \\
\text { K. Herrlinger, Hamburg (DGVS) }\end{array}$ \\
\hline & CC participants & $\begin{array}{l}\text { D. Bettenworth, Münster (DGVS) } \\
\text { M. Götz, Tübingen (DGVS) } \\
\text { U. Helwig, Oldenburg (DGVS) } \\
\text { L. Leifeld, Hildesheim (DGVS) } \\
\text { G. Moog, Kassel (DGVS) } \\
\text { E. Rijcken, Münster (DGAV/DGK) } \\
\text { F. Autschbach, Heilbronn (DGP) } \\
\text { G. Baretton, Dresden (DGP) } \\
\text { I. Kanbach, Berlin (DCCV) } \\
\text { S. Buderus, Bonn (GPGE) } \\
\text { P. Hartmann, Minden (FACED) }\end{array}$ \\
\hline \multirow[t]{2}{*}{$\begin{array}{l}\text { WG 2: medical management of active } \\
\text { ulcerative colitis }\end{array}$} & group leaders & $\begin{array}{l}\text { T. Kucharzik, Lüneburg (DGVS, KN-CED) } \\
\text { B. Siegmund, Berlin (DGVS, KN-CED) }{ }^{1}\end{array}$ \\
\hline & CC participants & $\begin{array}{l}\text { J. Büning, Lübeck (DGVS) } \\
\text { R. Ehehalt, Heidelberg (DGVS) } \\
\text { W. Häuser, Saarbrücken (DGVS) } \\
\text { F. Hartmann, Frankfurt (DGVS) } \\
\text { K. Kannengiesser, Lüneburg (DGVS) } \\
\text { K.-M. Keller, Wiesbaden (GPGE) } \\
\text { A. Lügering, Münster (DGVS) } \\
\text { S. In der Smitten, Berlin (DCCV) } \\
\text { J. Zemke, Herne (FACED) }\end{array}$ \\
\hline \multirow[t]{2}{*}{ WG 3: maintenance therapy } & group leaders & $\begin{array}{l}\text { A. Dignass, Frankfurt (DGVS) } \\
\text { S. Schreiber, Kiel (KN-CED, DGVS) }\end{array}$ \\
\hline & CC participants & $\begin{array}{l}\text { C. Maaser, Lüneburg (DGVS) } \\
\text { G. Rogler, Zürich (DGVS) } \\
\text { S. Koletzko, München (GPGE) } \\
\text { T. Kühbacher, Hamburg (DGVS) } \\
\text { W. Kruis, Köln (DGVS) } \\
\text { P. Esters, Frankfurt (DGVS) }\end{array}$ \\
\hline \multirow[t]{2}{*}{ WG 4: IBD-associated infections } & group leaders & $\begin{array}{l}\text { A. Stallmach, Jena (DGVS) }{ }^{1} \\
\text { N. Teich, Leipzig (DGVS) }\end{array}$ \\
\hline & CC participants & $\begin{array}{l}\text { M. Reinshagen, Braunschweig (DGVS) } \\
\text { T. Andus, Stuttgart (DGVS) } \\
\text { O. Bachmann, Hannover (DGVS) } \\
\text { M. Bläker, Hamburg (DGVS) } \\
\text { C. Veltkamp, Heidelberg (DGVS) }\end{array}$ \\
\hline \multirow[t]{2}{*}{ WG 5: surgery/Pouchitis } & group leaders & $\begin{array}{l}\text { P. Kienle, Heidelberg (DGAV/DGK) } \\
\text { A. Sturm, Berlin (DGVS) }\end{array}$ \\
\hline & CC participants & $\begin{array}{l}\text { S. Fichtner-Feigl, Freiburg (DGAV/DGCH/DGK)ํ } \\
\text { K. Fellermann, Lübeck (DGVS) } \\
\text { E. Stange, Stuttgart (DGVS) } \\
\text { A. Kroesen, Köln (DGAV/DGCH/DGK) } \\
\text { A. Pace, Neumünster (DGVS) } \\
\text { B. Kaltz, Berlin (DCCV) }\end{array}$ \\
\hline \multirow[t]{2}{*}{$\begin{array}{l}\text { AG 6: complementary medicine and } \\
\text { nutrition }\end{array}$} & group leaders & $\begin{array}{l}\text { J. Langhorst, Essen (DGVS) } \\
\text { J. Stein, Frankfurt (DGVS) }\end{array}$ \\
\hline & CC participants & $\begin{array}{l}\text { H. Matthes, Berlin (DGVS) } \\
\text { D.C. Baumgart, Berlin (DGVS) } \\
\text { J. Ockenga, Bremen (DGEM, DGVS) } \\
\text { J. Klaus, Ulm (DGVS) } \\
\text { C. Gross, Berlin (DCCV) }\end{array}$ \\
\hline coordinators & & $\begin{array}{l}\text { A. Dignass, Frankfurt (DGVS) } \\
\text { T. Kucharzik, Lüneburg (DGVS) }\end{array}$ \\
\hline
\end{tabular}


defined key questions, carried out a systematic literature review de novo and compiled evidence tables. Until the consensus conference took place, the literature could be supplemented by publications known to WG members which were not found during the systematic literature search. Recommendations for which no new evidence was available were taken unchanged from the old guidelines.

The literature review was conducted according to evidence classification of the Oxford Centre for Evidence-based Medicine 2011 ( $\triangleright$ Fig. 1).

Details on the search and selection and evaluation of evidence are presented in the guidelines report.

\section{Wording of recommendations and structure of consensus building}

On the basis of the literature search, selection and evaluation of the evidence, the recommendations and background texts were developed by the WGs and circulated by e-mail within the individual WGs until agreement was reached. When correlating the evidence strength to the level of recommendation, the recommendation grade could be up- or downgraded compared to the strength of the evidence for the reasons given in $\mathbf{F i g . ~} \mathbf{2}$. The graduation of the recommendations was also done on the formulation should, can ( $\vee$ Table 3 ).

All recommendations were agreed in a two-staged consensus process:

1. online-based consensus using the Delphi method

2. a moderated final consensus conference

Strength of consensus was classified as shown in $\$$ Table 4. Following the final consensus conference, the guidelines underwent final revision by the WG leaders and were structured and edited for publication by the coordinators.

\section{Statements}

Statements are descriptions or explanations of specific facts or questions without an immediate request for action. The statements may derive from either study data or expert opinion, and were agreed in accordance with the formal consensus procedure employed for the recommendations.

\section{Expert consensus}

Expert consensus refers to recommendations for which no systematic search for literature has been carried out or for which no literature is available after extensive research. As a rule, these recommendations address procedures of good clinical practice for which clinical studies are neither required nor available. While expert consensus was not graded using symbols, the strength of the recommendations is expressed by their wording (should, can) according to the gradation in $>$ Table 3.

Details regarding wording of recommendations and the structured consensus finding are described in the guidelines report.

\section{External evaluation and approval}

Adoption by the board of directors of the issuing professional societies and associations

Following the peer review process, the complete guideline was reviewed and agreed upon by all participating professional associations.

\section{Editorial independence and funding of the guideline}

Literature search, conferences and travel expenses were financed by the DGVS. There was no financial involvement of third parties. Mandate holders and experts worked exclusively on an honorary basis.

\section{Declaration and handling of conflicts of interest}

In accordance with regulations concerning the handling of conflicts of interest issued by the Association of the Scientific Medical Societies in Germany (Arbeitsgemeinschaft der Wissenschaftlichen Medizinischen Fachgesellschaften, AWMF), all involved persons submitted a declaration of their conflicts of interest on the official AWMF form prior to the consensus conference. Conflicts of interests were reviewed by the guideline coordinators and the DGVS (P. Lynen) and presented to the guidelines group before the consensus conference began. The elected representatives declared a large number of potential conflicts of interest. In the opinion of the guideline group, both the interdisciplinary nature of the involved parties (including representatives of the patient associations), and the entirely independently performed systematic search and evaluation of the literature served to offset any conflicts of interest of the individuals concerned. After critical assessment by the guidelines group, elected representatives who declared personal financial rewards (membership of advisory boards, consultancy or lecturing) were not excluded from the voting, provided the financial rewards were not unilateral (e. g. membership of several advisory boards) and the scientific expertise was indispensable. Payments exclusively attributable to scientific institutions (e. g. support grants, study funding) did not lead to exclusion from the voting. Representatives who did not declare their conflicts of interest and representatives who had ownership interests (e.g. patents, stocks or shares, company affiliation) were not entitled to vote. After thorough appraisal of all declared conflicts of interest, no representatives were excluded. Conflicts of interest are published in the guideline report.

\section{Distribution and implementation}

\section{Distribution and implementation concept}

The German guideline has been published in the German gastroenterology journal "Zeitschrift für Gastroenterologie", on the guidelines portal of the AWMF (www.awmf.org) and on the DGVS homepage (www.dgvs.de). The English translation is published in Pubmed (www.ncbi.nlm.nih.gov). A patient guideline prepared by the Gastroliga and the DCCV (www.dccv.de), and a compact version (synopsis), will also be made available. 


\begin{tabular}{|c|c|c|c|c|c|}
\hline Question & $\begin{array}{l}\text { Step } 1 \\
\text { (Level 1*) }\end{array}$ & $\begin{array}{l}\text { Step } 2 \\
\text { (Level 2*) }\end{array}$ & $\begin{array}{l}\text { Step } 3 \\
\text { (Level } 3^{*} \text { ) }\end{array}$ & $\begin{array}{l}\text { Step } 4 \\
\left(\text { Level } 4^{*} \text { ) }\right.\end{array}$ & Step 5 (Level 5) \\
\hline $\begin{array}{l}\text { How common is the } \\
\text { problem? }\end{array}$ & $\begin{array}{l}\text { Local and current random sample } \\
\text { surveys (or censuses) }\end{array}$ & $\begin{array}{l}\text { Systematic review of surveys } \\
\text { that allow matching to local } \\
\text { circumstances** }\end{array}$ & Local non-random sample & Case-series". & $\mathrm{h} / \mathrm{a}$ \\
\hline $\begin{array}{l}\text { Is this diagnostic or } \\
\text { monitoring test } \\
\text { accurate? } \\
\text { (Diagnosis) }\end{array}$ & $\begin{array}{l}\text { Systematic review } \\
\text { of cross sectional studies with } \\
\text { consistently applied reference } \\
\text { standard and blinding }\end{array}$ & $\begin{array}{l}\text { Individual cross sectional } \\
\text { studies with consistently } \\
\text { applied reference standard and } \\
\text { blinding }\end{array}$ & $\begin{array}{l}\text { Non-consecutive studies, or studies without } \\
\text { consistently applied reference standards** }\end{array}$ & $\begin{array}{l}\text { Case-control studies, or } \\
\text { "poor or non-independent } \\
\text { reference standard"* }\end{array}$ & $\begin{array}{l}\text { Mechanism-based } \\
\text { reasoning }\end{array}$ \\
\hline $\begin{array}{l}\text { What will happen if } \\
\text { we do not add a } \\
\text { therapy? } \\
\text { (Prognosis) }\end{array}$ & $\begin{array}{l}\text { Systematic review } \\
\text { of inception cohort studies }\end{array}$ & Inception cohort studies & Cohort study or control arm of randomized trial* & $\begin{array}{l}\text { Case-series or case- } \\
\text { control studies, or poor } \\
\text { quality prognostic cohort } \\
\text { study }\end{array}$ & $\mathrm{h} / \mathrm{a}$ \\
\hline $\begin{array}{l}\text { Does this } \\
\text { intervention help? } \\
\text { (Treatment Benefits) }\end{array}$ & $\begin{array}{l}\text { Systematic review } \\
\text { of randomized trials or } n-\text { of }-1 \text { trials }\end{array}$ & $\begin{array}{l}\text { Randomized trial } \\
\text { or observational study with } \\
\text { dramatic effect }\end{array}$ & $\begin{array}{l}\text { Non-randomized controlled cohort/follow-up } \\
\text { study*" }\end{array}$ & $\begin{array}{l}\text { Case-series, case-control } \\
\text { studies, or historically } \\
\text { controlled studies"* }\end{array}$ & $\begin{array}{l}\text { Mechanism-based } \\
\text { reasoning }\end{array}$ \\
\hline $\begin{array}{l}\text { What are the } \\
\text { COMMON harms? } \\
\text { (Treatment Harms) }\end{array}$ & $\begin{array}{l}\text { Systematic review of randomized } \\
\text { rrials, systematic review } \\
\text { of nested case-control studies, } n \text { - } \\
\text { of-1 trial with the patient you are } \\
\text { raising the question about, or } \\
\text { observational study with dramatic } \\
\text { effect }\end{array}$ & $\begin{array}{l}\text { Individual randomized trial } \\
\text { or (exceptionally) observational } \\
\text { study with dramatic effect }\end{array}$ & $\begin{array}{l}\text { Non-randomized controlled cohort/follow-up } \\
\text { study (post-marketing surveillance) provided } \\
\text { there are sufficient numbers to rule out a } \\
\text { common harm. (For long-term harms the } \\
\text { duration of follow-up must be sufficient.)*. }\end{array}$ & $\begin{array}{l}\text { Case-series, case-control, } \\
\text { or historically controlled } \\
\text { studies"* }\end{array}$ & $\begin{array}{l}\text { Mechanism-based } \\
\text { feasoning }\end{array}$ \\
\hline $\begin{array}{l}\text { What are the RARE } \\
\text { harms? } \\
\text { (Treatment Harms) }\end{array}$ & $\begin{array}{l}\text { Systematic review of randomized } \\
\text { trials or } n \text {-of-1 trial }\end{array}$ & $\begin{array}{l}\text { Randomized trial } \\
\text { or (exceptionally) observational } \\
\text { study with dramatic effect }\end{array}$ & & & \\
\hline $\begin{array}{l}\text { Is this (early } \\
\text { detection) test } \\
\text { worthwhile? } \\
\text { (Screening) }\end{array}$ & $\begin{array}{l}\text { Systematic review of randomized } \\
\text { trials }\end{array}$ & Randomized trial & $\begin{array}{l}\text { Non -randomized controlled cohort/follow-up } \\
\text { study"* }\end{array}$ & $\begin{array}{l}\text { Case-series, case-control, } \\
\text { or historically controlled } \\
\text { studies** }\end{array}$ & $\begin{array}{l}\text { Mechanism-based } \\
\text { reasoning }\end{array}$ \\
\hline
\end{tabular}

Fig. 1 CEBM Levels of Evidence 2011.

\section{Going from evidence to recommendations: Visualisation of considered judgment as a process of criteria-based consensus decision-making}

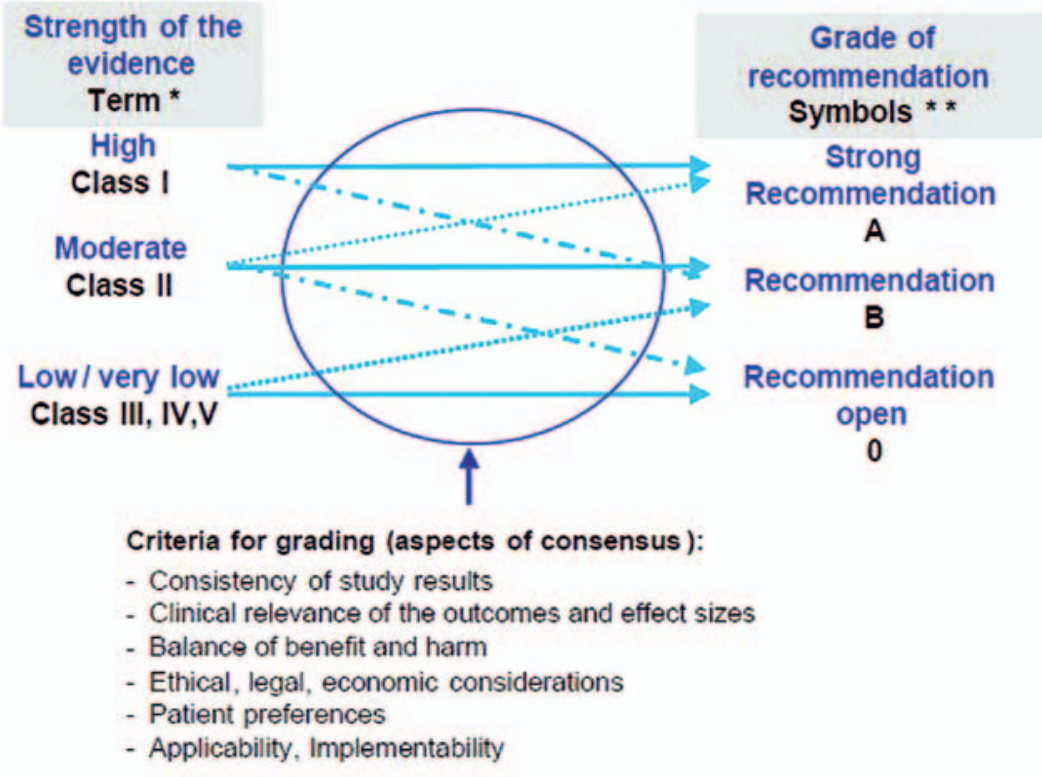

- Fig. 2 Diagram showing levels of evidence and the corresponding grades of recommendation.

\section{Validity period and updating procedures}

This updated guideline was most recently revised in May 2018. Its validity is estimated at approximately four years. The next revision process will be initiated by the DGVS guideline officer. Should there be important innovations in the diagnosis and therapy of ulcerative colitis in the meantime which appear to necessitate updating the guidelines, the guidelines coordinators will decide together with the steering group (B. Bokemeyer, P. Kienle, B. Siegmund, A. Stallmach) on the necessity and possible contents of an update. This will then be published online on the AWMF guideline portal and the DGVS homepage. 
- Table 3 Definition of classes of recommendation (A, B, C).

\begin{tabular}{|l|l|l|}
\hline $\begin{array}{l}\text { recommendation } \\
\text { grade (S3 only) }\end{array}$ & description & syntax \\
\hline A & strong recommendation & should \\
\hline B & recommendation & should \\
\hline C & neutral & can \\
\hline
\end{tabular}

- Table 4 Classification of degree of consensus.

\begin{tabular}{|l|l|}
\hline consensus & $\%$ agreement \\
\hline strong consensus & $>95$ \\
\hline consensus & $>75-95$ \\
\hline majority approval & $>50-75$ \\
\hline no consensus & $<50$ \\
\hline
\end{tabular}

\section{Editorial note}

\section{Gender neutrality}

In order to improve legibility, gender-specific language has not been used. All personal designations in this document are therefore to be understood as gender-neutral.

\section{Participatory decision-making}

All recommendations of the guideline are to be understood as recommendations intended to be discussed and implemented in the sense of a participative decision-making process involving the physician and the patient and, if necessary, the relatives.

\section{Special note}

Since the field of medicine is subject to a continual development process, all statements, especially those concerning diagnostics and therapeutic approaches, can reflect only the current knowledge base at the time of going to press. The greatest possible care has been taken with regard to recommendations given for therapy and the selection and dosage of medications. Nevertheless, guideline users are strongly advised to consult the package insert and the manufacturer's detailed product information and if in doubt, to contact a specialist. In the common interest, we would kindly request that any discrepancies or inconsistencies be reported to the editorial staff. The guidelines user remains personally responsible for every diagnostic and therapeutic application, and for the choice and dosage of medication.

Registered trademarks of products mentioned in these guidelines have not been specially indicated. Thus, if there is no indication that a product name is trademarked, it cannot be presumed unregistered.
This entire document in all its parts is protected by copyright. The use or exploitation of any part of the document other than as defined in copyright law, particularly its duplication, adaption, translation, microfilming, or its storage, processing or reproduction in electronic systems, intranets or the internet, is illegal and subject to prosecution, unless prior written permission is obtained from the DGVS.

\section{Diagnostics}

\section{Classification}

\section{RECOMMENDATION 2.1}

The disease should be classified according to its extent. Its localisation should be endoscopically classified as proctitis (limited to the rectum only), left-sided colitis (extending up to the left flexure) or extensive colitis.

Expert consensus, strong recommendation, strong consensus

\section{Background}

The classification of ulcerative colitis (UC) according to the extent of the disease is useful for two main reasons: Firstly, the disease location defines the choice of topical and/or systemic applications of the medication, especially with regard to 5-ASA preparations. In case of proctitis, suppositories are preferred, while enemas and foams can be used in left-sided colitis. Extensive colitis should be treated using oral medication which, according to the study of Marteau, should ideally be combined with an additional topical 5-ASA therapy [1]. Secondly, disease extent influences the necessity to commence endoscopic carcinoma screening. Thus, the recommendation for screening colonoscopy differs according to disease extent (see 2.28 -2.31). The preferred classification distinguishes three localisations; proctitis, left-sided colitis, and extensive colitis extending beyond the left flexure (Montreal Classification) [2] ( $\triangleright$ Table 5).

\section{RECOMMENDATION 2.2}

Concomitant PSC should be documented, since it influences the endoscopic surveillance strategy.

Expert consensus, strong recommendation, strong consensus

\section{Background}

Documentation of primary sclerosing cholangitis (PSC) is important, since it is associated with an increased risk for the development of colorectal carcinoma [3,4]. This is reflected in the colon carcinoma surveillance programme recommended for patients with ulcerative colitis and concomitant PSC (see 2.32). 
- Table 5 Extent of disease in ulcerative colitis (according to Silverberg et al. [2])

\begin{tabular}{|l|l|l|}
\hline classification & extent & description \\
\hline E1 & proctitis & $\begin{array}{l}\text { restricted to the rectum (distal } \\
\text { to the rectosigmoid junction) }\end{array}$ \\
\hline E2 & left-sided colitis & extending up to the left flexure \\
\hline E3 & extensive colitis & $\begin{array}{l}\text { extending beyond the left } \\
\text { flexure, including pancolitis }\end{array}$ \\
\hline
\end{tabular}

\section{Medical History}

\section{RECOMMENDATION 2.3}

The medical history should be based on detailed questioning on the types and onset dates of symptoms, recent travel history, nutritional allergies/intolerances, contact with infectious diarrhoeal diseases, vaccination status, smoking status, family medical history and medication history (especially concerning antibiotics and nonsteroidal antirheumatic drugs). In addition, the anamnesis should include questions covering extraintestinal manifestations (mouth, skin, eyes and/or joints), perianal abscesses, fistulae and anal fissures.

Expert consensus, Recommendation, Consensus

\section{Background}

In internal medicine, a comprehensive medical history and physical examination are of course a part of daily routine and not specific to patients with ulcerative colitis. There are nonetheless certain aspects which play a particularly important role in ulcerative colitis and therefore deserve special attention.

The distinction between Crohn's disease and ulcerative colitis can sometimes be difficult or impossible. The absence of rectal blood loss or symptoms in active smokers should be more reminiscent of Crohn's disease. Infectious or drug-induced colitis should be defined based on the medical history as far as possible. Use of nonsteroidal antirheumatic drugs (NSARs) appears to increase the risk of disease exacerbation in existing ulcerative colitis [5-8].

Almost half of patients with ulcerative colitis experience disease progression which necessitates the use of immunosuppressive therapies with steroids, thiopurines or TNF antibodies $[9,10]$. The risk of opportunistic infections under immunosuppressive therapy, especially under multiple immunosuppressants, is significantly increased [11]. Hence, assessment and completion of the recommended vaccination programme is advised both by ECCO, in a consensus statement, and by the DGVS in the present guideline.

Active smoking has a protective effect with regard to the development and severity of ulcerative colitis [12, 13]. However, ex-smokers have a $70 \%$ higher risk of developing ulcerative colitis, and more often suffer a refractory and extensive disease course, even compared with patients who have never smoked. Hospitalisation and colectomy rates are also higher in ex-smokers than in patients who have never smoked $[14,15]$. Ex-smokers who start smoking again appear to experience a milder disease course $[16,17]$. The question remains controversial as to whether smoking can prevent the occurrence of PSC or pouchitis after colectomy and ileoanal pouch construction $[18,19]$. Appendectomy also seems to play a role in the development of ulcerative colitis. Cohort studies and a meta-analysis indicate that childhood appendectomy (due to "genuine" appendicitis) has a protective effect (69\% risk reduction) with regard to the later development and severity of ulcerative colitis. Again, however, not all subsequent studies have confirmed these data [16, 20 - 25]. Nevertheless, it seems that appendectomy has a protective effect additive to the effect of smoking, whereas appendectomy does not hinder the development of PSC. Appendectomy after the onset of ulcerative colitis apparently has no further positive effect. Therefore, although the data on appendectomy are interesting from an epidemiologic and pathophysiological point of view, they have no therapeutic consequences in everyday clinical practice and are not considered in these recommendations.

Family medical history is of particular anamnestic importance. First degree relatives of patients with ulcerative colitis have a 10- to 15-fold increased risk of developing ulcerative colitis themselves [17]. However, the life-long risk of first-degree relatives for developing ulcerative colitis is only $5 \%$ (or conversely, $95 \%$ for not getting ulcerative colitis). This is valuable information for patients considering family planning. Family cases of ulcerative colitis seem to predominantly affect females; furthermore, first symptoms in these patients seem to occur at a younger age compared with sporadic cases [26].

\section{RECOMMENDATION 2.4}

At initial diagnosis and if specific symptoms occur, a complete physical examination should be performed, including oral and perianal inspection, and considering any extraintestinal manifestations. If not done previously, rectal examination should be performed during colonoscopy.

Expert consensus, strong recommendation, consensus

\section{Background}

In patients with ulcerative colitis who have no extraintestinal manifestations, physical examination is relatively unspecific. Clinical symptoms such as diarrhoea, tenesmus and rectal bleeding frequently predominate, while severe flares may additionally be characterised by tachycardia, weight loss, abdominal resistance and/or reduced bowel sounds. There was much debate, both in the working group and in the plenum, on the necessity for perianal inspection and rectal examination. These are, of course, not required at every patient consultation visit, but are generally performed during colonoscopy. In view of the increased risk of (colo-)rectal carcinoma in individuals with ulcerative colitis, however, the documentation of a rectal examination appears justified. Patients should be explicitly questioned concerning extraintestinal manifestations of the eyes, mouth, joints and skin, and also with regard to perianal manifestations [27]. 


\section{RECOMMENDATION 2.5}

In children and adolescents, the development of weight, height and puberty stage should be additionally documented at initial diagnosis and regularly during the course of the disease. Expert consensus, strong recommendation, strong consensus

\section{Background}

To facilitate the diagnosis of growth retardation in children and adolescents, the z-score or standard height-weight ratio in comparison to national gender-specific reference values is to be documented. In case of growth retardation, bone age is to be determined by means of an x-ray examination of the non-dominant hand. In addition, the puberty stage according to Tanner should be determined [28].

\section{RECOMMENDATION 2.6}

Diagnosis of ulcerative colitis should be based on a combination of medical history, clinical examination and typical laboratory, sonographic, endoscopic and histological findings. Expert consensus, strong recommendation, consensus

\section{RECOMMENDATION 2.7}

In case the diagnosis remains doubtful, endoscopy, including histological sample collection, should be repeated at an interval, e.g. after 3-6 months.

Evidence grade 4, recommendation grade $B$, consensus

\section{Background}

The natural course of ulcerative colitis is characterised by episodic disease flares, alternating with phases of remission. At presentation, it may be difficult to rule out infectious colitis, since only the disease course shows the chronic characteristics of the disease (see below). Rarely (only about $5 \%$ of patients) the course of disease can be continuous without intermittent remission phases. Equally prevalent is the manifestation of ulcerative colitis as a single flare with subsequent prolonged continuous remission [29]. Rapid establishment of the diagnosis, including the extent and severity of the relapse, enables an optimal therapeutic strategy. However, there is no gold standard for the diagnosis of ulcerative colitis. The diagnosis is based on the combination of typical findings in medical history, endoscopy, sonographic/radiological techniques and histopathology. Pathomorphological criteria are determined from biopsies collected during endoscopy or through examination of surgical specimens. If mucosal histopathology shows normal findings, active ulcerative colitis can be ruled out. Within 5 years of initial diagnosis, the diagnosis is amended in approximately $10 \%$ of patients to Crohn's disease, or the diagnosis of inflammatory bowel disease is discarded entirely. Therefore, especially if there are doubts concerning the diagnosis, it is advisable to repeat the endoscopic examination with histological sampling in order to gain endoscopic and histopathological confirmation [30]. In a minority of patients, an exact categorisation of the disease according to the entities of ulcerative colitis or Crohn's disease will not be possible, even in the long term. Such cases are described as "indeterminate colitis" (or, according to the Montreal Working Party 2005: Inflammatory Bowel Disease unclassified (IBDu)) [2, 31].

\section{Diagnosis}

\section{RECOMMENDATION 2.8}

Initial laboratory diagnostics should include at least the following parameters in addition to the blood count: inflammation status, iron status, renal function, transaminases and cholestasis parameters.

Evidence grade 4, recommendation grade B, strong consensus

\section{RECOMMENDATION 2.9}

When monitoring therapeutic response, CRP and/or faecal neutrophil markers can serve as laboratory parameters of disease progression.

Evidence grade 2, recommendation grade 0 , majority approval

\section{Background}

In every patient with ulcerative colitis, minimum laboratory diagnostics should include blood count, inflammatory markers (CRP), iron status parameters, renal retention parameters, transaminases and cholestasis parameters. In some cases, however, especially in mild to moderate ulcerative colitis and/or distal disease, laboratory values may be within the normal reference ranges. Unless disease is limited to proctitis (which is not usually associated with abnormal laboratory values), C-reactive protein correlates with the extent of disease and weakly with clinical activity [32, 33]. Although the CRP increase is generally less pronounced in patients with ulcerative colitis than in patients with Crohn's disease, in CRP-positive patients, it can provide a useful marker for clinical and endoscopic activity [34]. As a rule, patients with severe disease activity also show increased erythrocyte sedimentation rates (ESR) and anaemia. The significance of faecal stool markers, and in particular calprotectin, as markers of clinical and endoscopic inflammatory activity in ulcerative colitis, has been demonstrated in a number of studies [35-38]. However, neither serological markers such as CRP nor faecal inflammation markers can differentiate ulcerative colitis from an infectious cause. Two small studies report procalcitonin to be useful for the differentiation of self-limiting aetiologies of colitis [39, 40]. Especially during the initial diagnosis, stool cultures are helpful for differentiating and diagnosing self-limiting infectious colitis [41, 42] (see recommendation 2.10).

As a marker of iron status, ferritin is only of limited use, since it is an acute-phase protein and may therefore be increased in the presence of inflammation, irrespective of body iron stores. Therefore, in case of doubt, transferrin saturation and/or soluble trans- 
ferrin receptor levels should additionally be determined [43-46]. Due to their low sensitivity, routine determination of perinuclear anti-neutrophil cytoplasmic antibodies (pANCA) in ulcerative colitis and anti-Saccharomyces cerevisiae antibodies (ASCA) in Crohn's disease is not recommended as a means of differentiating the two disease entities [47]. Nevertheless, it may prove helpful in certain cases. In most of the literature, the reported prevalence of pANCA is up to $65 \%$ in patients with ulcerative colitis and less than $10 \%$ in patients with Crohn's disease [48, 49]. A large metaanalysis of 60 studies showed a sensitivity of $55 \%$ (specificity $93 \%$ ) for the constellation ASCA+/PANCA- for the detection of Crohn's disease, while the sensitivity of pANCA+ for detection of ulcerative colitis was found to be $55 \%$ (specificity $89 \%$ ) and even higher in paediatric patients (70\%/93\%) [50].

\section{RECOMMENDATION 2.10}

Intestinal infection should be excluded at initial diagnosis and whenever symptoms suggesting an acute flare arise during follow-up.

Evidence grade 4, recommendation grade B, consensus At initial diagnosis, microbiological stool diagnostics should be performed to detect bacterial infectious pathogens including Clostridium difficile toxin.

Evidence grade 2, recommendation grade B, consensus

In patients with appropriate travel history, complementary diagnostics should be carried out with respect to typical regional pathogens.

Evidence grade 4, recommendation grade B, consensus

\section{Background}

In the initial diagnosis, the differentiation from infectious causes, which are usually self-limiting, is important. Stool samples should be tested for common pathogens including Campylobacter spp, Escherichia coli 0157:H7 and Clostridium difficile toxins A and B. Depending on the medical history, special diagnostic stool tests may be useful, such as stool microscopy and stool antigen tests for amoebae or other parasites. Specific diagnostics will be addressed in detail in the chapter on infections (Chapter 4).

\section{RECOMMENDATION 2.11}

In the case of established ulcerative colitis, microbiological diagnostics including tests for Clostridium difficile toxin and cytomegalovirus should be carried out in the event of a severe disease flare or therapy-refractory course, and prior to intensification of immunosuppressive therapy.

Evidence grade 2, recommendation grade A, strong consensus

\section{Background}

In the further course, stool diagnosis does not have to be repeated with every relapse $[42,51]$; however, in particular, diagnostic tests for $C$. difficile [52 - 54] and cytomegalovirus (CMV) (re-)infection [55-58] should be performed if disease is severe or refractory, or if the flare was anamnestically preceded by intake of antibiotics (see also Chapter 4: infections).

\section{RECOMMENDATION 2.12}

Quantitative determination of faecal neutrophil markers (e. g., calprotectin) should be included in clinical differential diagnostics to distinguish symptoms from (functional) symptoms of irritable bowel syndrome.

Evidence grade 2, recommendation grade $B$, consensus

\section{RECOMMENDATION 2.13}

For the diagnostic monitoring of established ulcerative colitis, the quantitative determination of faecal neutrophil markers should be used.

Evidence grade 1, recommendation grade $B$, strong consensus

\section{Background}

A number of different faecal inflammatory markers have been investigated in inflammatory bowel disease, including calprotectin, lysozyme, PMN elastase, lactoferrin and S100A12 [59-62]. Calprotectin and lactoferrin seem to offer the highest sensitivity as markers of intestinal inflammation, and correlate with the clinical and endoscopic severity of inflammation in ulcerative colitis $[63,64]$. Faecal markers are, however, of limited diagnostic value in the primary diagnosis of ulcerative colitis, being unable to distinguish between different causes of intestinal inflammation. Nevertheless, faecal markers are helpful in differentiating functional symptoms and especially in paediatric diagnostics. The results of several trials have shown that faecal calprotectin levels are significantly higher in both paediatric and adult patients with inflammatory bowel disease than in healthy controls or patients with irritable bowel syndrome. The markers can therefore be used very well as differentiation markers for irritable bowel syndrome or for non-inflammatory causes of intestinal complaints, especially in paediatrics [65-68].

Furthermore, several studies have shown that calprotectin is a highly sensitive and specific indicator of endoscopic disease activity in ulcerative colitis even before the onset of clinical symptoms, making it a reliable early marker of disease relapse [37, 38, $69,70]$. Faecal neutrophil markers can therefore aid the assessment of patient symptoms in daily practice. In addition, the individual longitudinal profiles of neutrophil markers provide a good overview of disease activity.

\section{Endoscopic Diagnostics}

\section{RECOMMENDATION 2.14}

If ulcerative colitis is suspected, an ileocolonoscopy with biopsies from the terminal ileum and all colon segments including the rectum (at least 2 biopsies per segment, submission in 
separate sample tubes) should be performed to confirm the diagnosis and to determine the extent of the disease.

Evidence grade 4, recommendation grade B, consensus

\section{Background}

Full colonoscopy, with intubation of the terminal ileum and segmental extraction of intestinal biopsies, is preferable to sigmoidoscopy in the initial diagnosis of patients with suspected ulcerative colitis, since it enables the determination of disease localisation and extent, and to a large degree, the exclusion of Crohn's terminal ileitis [71, 72]. This approach appears to be more cost-effective than index sigmoidoscopy [73, 74].

During the initial diagnosis, at least two biopsies each should be obtained from the ileum and all colon segments, including the rectum. The tissue specimens should be labelled separately according to their localisation. If diagnosis is uncertain, subsequent endoscopic re-evaluation with appropriate histopathological assessment may be required. It has been reported that in approximately $10 \%$ of patients, the initial diagnosis of ulcerative colitis is retracted or changed to Crohn's disease within five years [75]. In patients with acute severe colitis, complete colonoscopy is not recommended and should first be replaced by sigmoidoscopy.

\section{RECOMMENDATION 2.15}

Routine colonoscopy should not be carried out in patients with ulcerative colitis in remission until carcinoma surveillance is required.

Expert consensus, recommendation, strong consensus

Endoscopic re-evaluation can be considered as a means of assessing therapy response and for decision-making with regard to therapy de-escalation in patients receiving immunosuppressive or biological therapies.

Expert consensus, recommendation open, strong consensus

\section{RECOMMENDATION 2.16}

Endoscopic evaluation may be performed in patients with therapy-refractory disease to confirm disease activity and rule out infectious or other complications.

Expert consensus, recommendation open, consensus

\section{Background}

Despite the importance of assessing the extent of the disease in determining the prognosis, the need for monitoring and the choice of therapy, the adequacy of regular repeat examinations after index colonoscopy has not yet been investigated. Drug-induced clinical remission is not necessarily associated with endoscopic and histological remission [76]. There are numerous indications that so-called mucosal healing is associated with a more favourable clinical outcome [77]. Systematic reviews have demonstrated an association of mucosal healing with avoidance of colectomy and the attainment of steroid-free clinical remission $[78,79]$. Therefore, endoscopic re-assessment may be considered in patients who achieve a drug-induced clinical remission.

Likewise, assessment of mucosal healing can be integrated into the decision-making process regarding therapy de-escalation in patients treated with immunosuppressants or biologicals who are in stable clinical remission. Although corresponding data in ulcerative colitis are sparse, results in Crohn's disease have been promising [80].

Numerous indices are available for the clinical or endoscopic assessment of disease activity. These indices are rarely used in daily routine, but are used in clinical trials. While endoscopic activity is commonly assessed using the endoscopic Mayo Score [81], the only validated endoscopic activity index is the Ulcerative Colitis Endoscopic Index of Severity (UCEIS) [82].

To date, the clinical classification of severe ulcerative colitis is widely based on Truelove and Witts' classification dating from 1955 [83], since it is easy to remember and simple to use. This classification is still considered the method of choice to identify patients in need of immediate hospitalisation and intensive therapy [84]. In paediatric patients, the PUCAI according to Turner has become established [85].

\section{Diagnostic differentiation from Crohn's disease}

\section{RECOMMENDATION 2.17}

If colitis cannot be clearly classified, the upper gastrointestinal tract should be examined by eosophagogastroduodenoscopy (with biopsies) and the mid-gastrointestinal tract by MRI of the small bowel and/or abdominal sonography.

Expert consensus, recommendation, strong consensus

\section{Background}

If diagnostic ambiguities are present (e. g., disease-free rectum, unusual symptoms, endoscopic evidence of backwash ileitis), the presence of Crohn's disease should be considered for differential diagnosis and, depending on the clinical context, appropriate diagnostics of the upper and middle digestive tract should be performed. Diagnostic procedures should be performed analogous to the current DGVS guidelines for the diagnosis and therapy of Crohn's disease [86].

\section{Ultrasound}

\section{RECOMMENDATION 2.18}

High-resolution abdominal sonography should be an integral part of initial and follow-up diagnostics, and should be used to detect complications in patients with severe relapse.

Evidence grade 2, recommendation grade B, strong consensus

\section{Background}

Transabdominal ultrasound can detect inflammation of the colon with a sensitivity of up to $90 \%$. Moreover, sonography is inexpensive and non-invasive. As with all diagnostic procedures, 
however, its precision depends upon the experience of the examiner. In addition, there is a low specificity to differentiate ulcerative colitis from other causes of colitis [87-89]. In the hands of an experienced sonographer, both the activity and the extent of disease can be reliably determined [90-96]. Sonography correlates well with endoscopic activity and can be used as a prognostic tool due to its good correlation with therapy response [97]. Doppler sonography of the superior and inferior mesenteric arteries has been used to evaluate disease activity and the risk of relapse. However, due to insufficient data and limited dissemination of the technique, it should not be considered a standard procedure at the present time $[98,99]$. For contrast-enhanced ultrasound examination (CEUS), a correlation with histological inflammatory activity has been demonstrated, but due to the insufficient data currently available, no recommendation for its use as a standard procedure can be given [100].

\section{Colon stenosis in ulcerative colitis}

\section{RECOMMENDATION 2.19}

Since the presence of a colonic stenosis in ulcerative colitis is suspicious for malignancy, ample biopsies should be taken from the area of the stenosis and additional diagnostic imaging (e.g., CT, MRI) performed. If the dignity of a colonic stenosis is unclear, the decision to perform surgery should be made generously.

Evidence grade 4, recommendation grade B, consensus

\section{Background}

In patients with long-standing ulcerative colitis, colonic stricture or stenosis must be interpreted as a sign of colorectal carcinoma; therefore, histologic evaluation is necessary [101]. Due to the submucosal growth of the ulcerative colitis-associated carcinoma, definitive endoscopic-histological clarification is often problematic [102, 103]. For this reason, it seems justifiable to recommend a generous stance concerning the indication for surgery. If colonoscopy is incomplete due to the presence of a stenosis or stricture, CT- or MR-colonography should be performed. CT colonography or MR colonography can identify the structure of the mucosa and the extent of the colitis proximal to the stricture, but may not show all lesions discernible during colonoscopy $[104,105]$. In a retrospective cohort study of patients with colonic stenosis in Crohn's disease and ulcerative colitis who had no preoperative signs of malignancy, colon carcinoma was diagnosed postoperatively in $7.8 \%$ of patients with ulcerative colitis [106]. Therefore, if findings are inconclusive, surgical resection should be performed. In spite of the sparse evidence, this recommendation has been made here to avoid any delay in surgery, in view of the possibility of colon carcinoma [106].

\section{Paediatrics}

\section{RECOMMENDATION 2.20}

The diagnosis of ulcerative colitis should be considered in children presenting with chronic ( $>4$ weeks) or recurrent ( $>2$ episodes within 6 months) bloody diarrhoea after infectious causes have been ruled out.

Evidence grade 4, recommendation grade B, consensus

\section{Background}

Children with ulcerative colitis generally show typical symptoms such as anaemia (84\%), chronic diarrhoea (74\%) and abdominal pain, predominantly in the form of tenesmus (62\%) [107]. Diagnostics should be performed in children with chronic (>2 weeks) or recurring diarrhoea, regardless of whether or not blood is passed in the stools. Weight loss is less typical of ulcerative colitis (35\%) than of Crohn's disease (58\%). The most common extraintestinal symptom is arthropathy (10\%). Manifestations affecting the skin are rare. In contrast to adult patients, three quarters of paediatric patients present with extensive ulcerative colitis, whereas distal disease is uncommon. Over $50 \%$ of paediatric patients with ulcerative colitis relapse at least once a year. The diagnosis of inflammatory bowel disease is confirmed by means of clinical evaluation in combination with biochemical, endoscopic, histological and, if Crohn's disease is suspected, radiological examinations (MRI-enterography or -enteroclysis). The diagnostic criteria correspond to those of adult patients [108].

\section{RECOMMENDATION 2.21}

Initial diagnostics in children and adolescents with suspected inflammatory bowel disease should include ileocolonoscopy with stepwise biopsies from the terminal ileum and all colon segments.

Evidence grade 4, recommendation grade A, strong consensus An oesophagogastroduodenoscopy with stepwise biopsies should be performed in the same examination procedure. Evidence grade 4, recommendation grade $B$, strong consensus

\section{Background}

The working group of the European Society of Pediatric Gastroenterology, Hepatology and Nutrition (ESPGHAN) has defined recommendations for the diagnostic procedure [109]. Children suspected of having inflammatory bowel disease should undergo full colonoscopy with intubation of the terminal ileum and stepwise extraction of biopsies (terminal ileum, coecum, colon ascendens, colon transversum, colon descendens, sigma and rectum) and oesophagogastroduodenoscopy with stepwise biopsies from oesophagus, stomach and duodenum [109]. Imaging of the small bowel (usually MRI-enterography) can only be dispensed with if symptoms are clearly assigned to ulcerative colitis. Since, in contrast to the adult patient population, three quarters of paediatric patients have extensive colitis, full colonoscopy is obligatory. Sig- 
moidoscopy alone is generally not indicated except in severe ulcerative colitis, which is associated with an increased risk of bowel perforation. In paediatric patients, endoscopy should be carried out under general anaesthesia or deep analgosedation. In paediatric patients with disease onset during the first three years of life, or perianal lesions, or a conspicuous history of frequent or unusual infections (regardless of age), and who have consanguineous parents, immunological investigations should be performed to rule out congenital immunodeficiency conditions as the cause of the colitis. Allergic colitis or nutritional allergy as a modulating factor of inflammatory activity should be considered in the differential diagnosis, especially in younger children with colitis.

\section{Histopathological diagnostics - inflammation diagnostics}

\section{RECOMMENDATION 2.22}

Histopathological criteria which should be used for the evaluation of biopsies for the diagnosis of ulcerative colitis are:

- diffuse panmucosal chronic inflammation (lymphocytes and plasma cells) in combination with impairment of the crypt architecture/crypt atrophy

- Plasmocytosis in the basal mucosal stroma

- Paneth cell metaplasia distal of the right colic (hepatic) flexure

- Reduced proliferation of goblet cells or reduced mucin content of the individual cells, continuous pattern of inflammatory and structural changes in the mucosa, decreasing gradient of inflammation from distal to proximal

Evidence grade 1, recommendation grade B, strong consensus

\section{RECOMMENDATION 2.23}

Deviating morphological patterns may occur in ulcerative colitis and should be considered especially in paediatric patients. Evidence grade 1, recommendation grade A, consensus

RECOMMENDATION 2.24

The pathological report should include a statement on histological inflammatory activity.

Expert consensus, recommendation, consensus

\section{Background}

The diagnosis of ulcerative colitis is based on evidence of a characteristic combination of clinical, biochemical, endoscopic, radiologic and pathomorphological findings. There is no gold standard for diagnosis. Pathohistological diagnostics rely on the synoptic evaluation of a combination of factors primarily relating to the type and distribution of the inflammatory infiltrates, and changes in the mucosal architecture [110-123]. The individual histological findings are not specific in themselves and some can also occur in other forms of inflammatory bowel disease.

Typical changes in the mucosal architecture in ulcerative colitis are irregularities in shape, orientation and size of the crypts (> $10 \%$ of the crypts; more than 2 branched, non-parallel crypts in one biopsy) $[117,119,121,123]$. The term crypt atrophy is used to describe a reduced crypt density (i. e. a distance greater than the cross-section of one crypt between 2 neighbouring crypts) and/or a displacement between the crypt base and the lamina muscularis mucosae, mostly in association with a basal increase of mononuclear, plasma cell-rich infiltrate $[115,116,119$, 121]. Transmucosal inflammation refers to a diffuse increase in the number of mononuclear cells in the lamina propria including the middle and basal mucosa layers [115, 119]. Basal plasmacytosis is defined as evidence of plasma cells in the basal region $(1 / 5)$ of the lamina propria or between the crypt base and the lamina muscularis mucosae (subcryptal) [116, 117].

The bioptic diagnosis, when used to distinguish ulcerative colitis from Crohn's disease and other inflammatory bowel diseases, relies on assessment of the extent (pronounced, diffuse) and the topographical distribution (continuous distribution, decreasing gradient from distal to proximal) of the histopathological criteria described in recommendation 2.22. Therefore, during the initial colonoscopy, biopsies should be taken stepwise from the terminal ileum and from all colon segments including the rectum, and the bioptic samples labelled according to their localisation. In this way, the diagnostic value of the samples can be greatly increased in comparison to single or unsystematic biopsies [111, 112, 124 127]. In order to rule out findings relevant for differential diagnosis (e. g., epithelioid cell granuloma), the analysis of samples in stepwise- or serial sections is recommended [128, 129].

When performed as described above, histopathological diagnosis of ulcerative colitis based on mucosal biopsies has a sensitivity and specificity of over $70 \%[111-113,118,123]$, in a number of studies up to more than $90 \%$ [120 - 122], at least in the presence of active disease. Several authors have proposed the use of standardised scoring models with numerical coefficients [120122], in which evidence of two to three of the described histomorphological criteria is required for diagnosis.

In the initial stages of disease ( $<4-6$ weeks), impairment of the crypt architecture may be absent, with the result that specific diagnosis may not be possible. In this context, the detection of basal plasmacytosis can be regarded as an early sign of potential inflammatory bowel disease [116, 117, 130, 131].

From a diagnostic point of view, it must be taken into account that the morphological appearance of inflammatory bowel disease is subject to biological variations (episodic progression) and is also influenced by therapy $[132,133]$. Thus, in the course of ulcerative colitis, a discontinuous pattern of inflammation may well occur, sometimes with sparing of the rectum [134]. Atypical manifestations are particularly common in paediatric IBD patients (younger than 10 years), even without prior therapy [135-138]. Information on the overall clinical picture (medical history, disease duration, type and duration of therapy, endoscopic findings) is therefore necessary for efficient assessment and improves the validity of the histological evaluation [112]. This applies particularly to the diagnostic differentiation of ulcerative colitis from 
other aetiologically or phenotypically defined forms of inflammation (infectious colitis, drug-induced colitis, diversion colitis, diverticular disease-associated colitis, allergy-associated colitis etc.) [139-141]. The histopathological classification of inflammatory activity in ulcerative colitis is based on the degree of tissue infiltration by segmented neutrophil granulocytes and associated damage to the intestinal epithelium, including epithelial invasion by neutrophils and the development of cryptic lesions and crypt abscesses, culminating in erosive and ulcerous lesions $[115,118$, $119,121]$. While histological findings and clinical disease activity correlate only to a limited extent in the individual patient [142], therapeutic trials have shown that clinical improvement is associated with the abatement of active histological changes [143]. An active morphological picture is associated with relapsing-remitting disease flares [144-146]. The pathologist's evaluation of histological inflammatory activity is therefore of particular interest with regard to the assessment of disease progression. Numerous indices have become established (e. g., Riley score). The only scores which have yet been validated are the Nancy histological index and the Robarts histopathology index [147, 148]. However, due to the heterogeneity of the numerous available scores, no specific histological index is presently recommended. The potential value of histopathology as a predictor of relapse and in monitoring the effectiveness of anti-inflammatory therapy has implications for therapeutic management and risk reduction with regard to neoplasia.

\section{Intraepithelial Neoplasia (IEN)}

\section{RECOMMENDATION 2.25}

The diagnosis of intraepithelial neoplasia/dysplasia in ulcerative colitis should be carried out according to current WHO criteria; IEN/dysplasia should be histopathologically classified as low-grade, high-grade or indefinite.

Expert consensus, strong recommendation, strong consensus

\section{RECOMMENDATION 2.26}

In the histological diagnosis of each IEN/dysplasia, a competent (documented) pathological second opinion in the sense of a four-eyes principle should be ensured.

Evidence grade 3, recommendation grade $B$, consensus

\section{Background}

Intraepithelial neoplasia/dysplasia is defined as a clearly neoplastic lesion of the epithelium which is confined to the basement membrane and shows no invasion into the lamina propria [149]. Dysplasia is the most reliable marker for an increased risk of intestinal malignancy [150]. Intraepithelial neoplasia/dysplasia is histologically classified according to the grade of neoplastic transformation as low-grade, high-grade or indefinite [149]. The identification of IEN is hindered by the high variability of individual pathologists' evaluations [151 - 153] with a K-value of 0.4 [154], whereby the variability in low-grade and indefinite IEN is especially high. In view of the profound therapeutic consequences, every histopathological diagnosis of "IEN/dysplasia" should be confirmed by another experienced pathologist in a second evaluation.

\section{RECOMMENDATION 2.27}

Any discernible lesion with IEN/dysplasia should be categorised by the endoscopist as a polypoid or non-polypoid IBD-associated lesion, and the grade of IEN/dysplasia (LGIEN or HGIEN) defined, since this classification is of therapeutic importance. Evidence grade 3, recommendation grade $B$, consensus

\section{Background}

In accordance with the recommendations of ECCO $[155,156]$ and the international SCENIC consensus conference, the macroscopic descriptions of IEN/dysplasia as DALM (dysplasia-associated lesion or mass), ALM (adenoma-associated lesion or mass), adenoma-like, non-adenoma-like, and flat will no longer be used, because these macroscopic criteria have often been used to describe diversely shaped lesions. Visual lesions should therefore be classified as polypoid or non-polypoid [157].

A polypoid lesion is defined as a pedunculated (Paris Classification Type $1 \mathrm{p}$ ) or sessile (Paris Classification Type $1 \mathrm{~s}$ ) lesion that protrudes from the mucosa into the lumen (>2.5 mm) [157]. These lesions can normally be removed by means of endoscopic resection [158].

Non-polypoid lesions are lesions classified as Paris Classification Type Ila (flat-elevated), Paris classification Type Ilb (flat-flat) and Paris Classification Type Ilc (flat-depressed). These include velvety patches, plaques, irregular bumps and nodules, thickening, stricturing lesions and broad-based masses. These lesions are not always removable by endoscopic resection [159-162]. Lesions are differentiated according to their endoscopic appearance. The term endoscopically invisible dysplastic lesion is used to describe a histologically evident IEN/dysplasia not visible during endoscopy.

\section{Surveillance colonoscopy}

\section{RECOMMENDATION 2.28}

Since colitis-associated colon cancer mortality can be reduced by surveillance colonoscopy, surveillance colonoscopies should be scheduled according to risk stratification.

Evidence grade 3, recommendation grade B, consensus

\section{Background}

The effectiveness of surveillance programmes has not yet been investigated in randomised controlled trials. However, a large number of published case control studies and case series endorse the benefit of screening colonoscopy [163 - 167]. Three case control studies examined the effectiveness of surveillance colonoscopy in ulcerative colitis [168-170]. A meta-analysis of these three 
studies and two additional studies showed that surveillance colonoscopy was associated with reduced mortality in patients with ulcerative colitis [171]. However, the evidence quality of the evaluated studies is considered to be poor.

\section{RECOMMENDATION 2.29}

In order to establish the surveillance strategy, all patients with ulcerative colitis, irrespective of disease activity, should undergo surveillance colonoscopy $6-8$ years after initial symptoms/diagnosis, with at least two biopsies from each colon segment in addition to targeted biopsy specimens to determine the extent/localisation of inflammation.

Evidence grade 2, recommendation grade $B$, strong consensus

\section{Background}

Until recently, it was widely accepted that colorectal carcinomas rarely occur during the first eight years after disease onset. However, trials have shown that a significant proportion of colorectal carcinomas appear prior to the eighth year of disease $[172,173]$. One study from the Netherlands demonstrated that in $22 \%$ of patients with ulcerative colitis who developed a colorectal carcinoma, the tumour occurred prior to beginning surveillance colonoscopy [174]. As a result of these data, it is recommended to perform a surveillance colonoscopy between the $6^{\text {th }}$ and $8^{\text {th }}$ year of disease, regardless of disease activity, in order to histologically assess the extent of disease and thereby establish a time schedule for the surveillance programme.

\section{RECOMMENDATION 2.30}

Patients with disease activity restricted to the rectum and without evidence of prior or current endoscopic and/or microscopic inflammation proximal to the rectum should not be included in a regular surveillance colonoscopy programme.

Expert consensus, recommendation, strong consensus

For disease monitoring, and in order to avoid overlooking any increase in the extent of ulcerative colitis, a surveillance colonoscopy every 5 years can be considered.

Expert consensus, recommendation open, strong consensus

\section{RECOMMENDATION 2.31}

In patients with inflammatory activity extending beyond the rectum, regular surveillance colonoscopy should be performed from the $8^{\text {th }}$ year after disease onset.

Expert consensus, strong recommendation, consensus

The surveillance strategy should be individually adapted and the interval should be based on risk stratification, as follows: high-risk patients (stenosis, IEN within the previous 5 years, extensive colitis with severe inflammation or first-grade relative with $C R C<50$ yrs.) should undergo surveillance colonoscopy annually, patients with intermediate risk (colitis with mild or moderate inflammation, numerous pseudopolyps, first-grade relative with $C R C \geq 50$ yrs.) every $2-3$ years, and low-risk patients (with none of the factors mentioned above) every 4 years.

Expert consensus, recommendation, consensus

\section{Background}

Patients with ulcerative colitis have an increased risk of colon carcinoma compared to the general population. The individual risk depends on the extent of disease. Various studies have shown that the risk is markedly increased in patients with extensive colitis and still distinctly increased in patients with left-sided colitis, but not clearly increased in patients with ulcerative proctitis [175].

While numerous studies have demonstrated an increased risk for colorectal carcinoma (CRC) in patients with ulcerative colitis, their conclusions differ as to the magnitude of the risk. Whereas earlier centre-based studies - summarised in a meta-analysis indicated considerably higher figures (CRC risk in UC patients $2 \%$ after 10 years; $8 \%$ after 20 years; and $18 \%$ after 30 years) [175], recent population-based studies showed only slightly increased rates (CRC risk $0.6-1.2 \%$ after 10 years; $2.1-5.4 \%$ after 20 years; and $4.7-7.5 \%$ after 30 years) $[176,177]$. Only in Denmark there was no increased incidence of CRC in UC patients, possibly due to a higher colectomy rate in the observation period [172]. Frequency correlated with disease duration, disease extent and inflammatory activity/pseudopolyps [3, 175, 178 - 181].

In a meta-analysis of 116 studies published in 2001, Eaden calculated a cumulative risk of $18 \%$ for colitis-associated carcinoma after 30 years [175]. Another meta-analysis described a 2.4-fold increase in rectal carcinoma in patients with ulcerative colitis [182]. However, more recent research seems to suggest that the risk of colorectal carcinoma may be decreasing. A Danish study by Jess in 2013 found no increased risk for CRC in UC patients, with a factor of $1.12(0.97-1.28)$, and in a case series in 2015, Choi described an only slightly increased CRC risk ( $0.1 \%$ after 10 yrs., $2.9 \%$ after 20 yrs., and $6.7 \%$ after 30 yrs.) [183, 184].

A more recent Australian study [185] indicated a cumulative risk of colorectal carcinoma in patients with ulcerative colitis of $1 \%$ after 10 years, $3 \%$ after 20 years and $7 \%$ after 30 years. This may reflect, on the one hand, the increasing implementation of surveillance programmes, and on the other, the growing effectiveness of anti-inflammatory drug therapies [186].

The aim of surveillance colonoscopy is to detect neoplasms with high sensitivity and specificity. This necessitates that the intestine is without significant inflammation which could be misinterpreted histologically as intraepithelial neoplasia. Analogous to screening colonoscopies in the normal population, it can be assumed that the quality of colonoscopy preparation significantly influences the detection rate of lesions [187]. Equally, there is a correlation between withdrawal time and the rate of detection of neoplasia [188]. As an innovation in this guideline, it is recommended that the monitoring interval for screening colonoscopy should be adapted according to risk stratification. For this purpose, various evidence-based risk constellations have been defined ( $\triangleright$ Table 6 ). Adjusted according to these risk constellations, the monitoring interval thereafter is 
- Table 6 Monitoring interval for surveillance colonoscopy according to risk stratification.

monitoring interval for surveillance colonoscopy from year $\mathbf{8}$ after disease onset according to risk stratification in ulcerative colitis (applicable is the highest risk for which one criterion is met), as described in statements 2.31 and 2.32 .

\begin{tabular}{|l|l|l|l|l|l|l}
\hline annually (high risk) & every $\mathbf{2 - 3}$ years (intermediate risk) & every $\mathbf{4}$ years (low risk) \\
\hline extensive colitis with severe inflammation & colitis with mild to moderate inflammation & $\begin{array}{l}\text { none of the criteria for high or intermediate } \\
\text { risk are present }\end{array}$ \\
\hline first-degree relative with CRC <50 years & first-degree relative with CRC $\geq 50$ years \\
\hline IEN in the previous 5 years & numerous pseudopolyps \\
\hline PSC (annually from time of diagnosis) & \\
\hline Stenosis & \\
\hline
\end{tabular}

1 year, 2 - 3 years or 4 years, depending on the described risk constellation [4, 152, 159, 178, 180, 186, 189 - 198].

Some reports suggest that certain additional risk factors, such as a colon carcinoma in the family history, the presence of backwash ileitis, or first manifestation of colitis in childhood or adolescence, may also play a role $[175,181,199]$. However, the available data are inconsistent.

\section{RECOMMENDATION 2.32}

In patients with concomitant PSC, surveillance colonoscopies should be carried out annually from the time of PSC diagnosis, regardless of the disease activity and extent of ulcerative colitis. Evidence grade 3 , recommendation grade $B$, consensus

\section{Background}

Patients with concomitant primary sclerosing cholangitis (PSC) are a particularly high-risk group. One meta-analysis calculated a five-fold increase in the risk of carcinoma $[4,191]$. These patients should therefore undergo regular annual surveillance colonoscopy from the time of PSC diagnosis. Other investigations have shown not only that the risk of carcinoma in UC patients with PSC is five times higher [4], but also that it develops earlier [152] and is more frequently located in the right-sided colon [191].

\section{RECOMMENDATION 2.33}

Surveillance colonoscopy with biopsy should, if possible, be carried out in the remission phase, since the histomorphological differentiation of inflammatory from neoplastic changes may otherwise be difficult.

Evidence grade 4, recommendation $B$, consensus

\section{Background}

If the colon is not in a largely inflammation-free state, inflammatory changes may show similarities to IEN. The pathologist may have difficulty discerning IEN with certainty in samples affected by active inflammation. Therefore, if a lesion is judged by the pa- thologist to be possibly suspicious for IEN, a follow-up endoscopy within 3 months (following intensification of anti-inflammatory therapy, if appropriate) may be required.

\section{RECOMMENDATION 2.34}

Targeted biopsies should be taken from all endoscopically suspect lesions. The surveillance colonoscopy should be performed in a clean intestine with sufficient withdrawal time. Evidence grade 1, recommendation grade $B$, consensus

\section{RECOMMENDATION 2.35}

Ideally, surveillance colonoscopy should be performed as chromoendoscopy with targeted biopsies, without additional random biopsies. Alternatively, high-definition white light endoscopy (HDWLE) with targeted biopsies of any visible lesion, without additional random biopsies, ensuring a high degree of meticulousness and appropriate withdrawal time, can be performed.

Evidence grade 1 , recommendation grade $B$, consensus

If HDWLE is not available, untargeted stepwise biopsies should additionally be collected.

Evidence grade 2, recommendation grade A, consensus

\section{Background}

For many years, there has been much discussion regarding the necessity for additional random biopsies and/or the use of chromoendoscopy in surveillance colonoscopy. Chromoendoscopy has become the established surveillance technique, achieving good results even without additional random sampling. Chromoendoscopy is therefore recommended as the preferred monitoring technique, with targeted biopsies but without the collection of random biopsies. Numerous studies are available [200-206]. However, only in recent investigations has the performance of chromoendoscopy in the detection of dysplasia been compared with that of high-definition endoscopes without chromoendoscopy [206-210]. The 
development of "high resolution" techniques over the past few years has greatly increased the detection rate for dysplasia [211].

A recently-published study from Japan [209] demonstrated that, in high-resolution colonoscopy, targeted biopsies alone are noninferior to targeted plus additional random biopsies. Additional studies in dysplasia diagnostics show that random samples in the context of a high-resolution colonoscopy system show no benefit compared to an examination performed with targeted biopsies only. In a systematic review from Italy pooling data from three randomised studies including a total of 190 patients [207], no difference in dysplasia detection rates was found between chromoendoscopy and high-definition white light endoscopy (HDWLE). However, in the comparison between chromoendoscopy and non-high-definition white light endoscopy (WLE), a significant difference was observed. Similar findings were reported from a unicentric trial from Canada [208] evaluating dysplasia detection in 454 IBD patients from a surveillance programme. The results indicated that detection of dysplasia using both HDWLE and chromoendoscopy was more effective using targeted biopsies than random biopsies. However, this result was not found in standard white light endoscopy (WLE). A randomised, multicentre study from Japan in 246 UC patients compared rates of dysplasia detection using random biopsies versus targeted biopsies, both predominantly taken during high resolution endoscopy (HDWLE). Intraepithelial neoplasia (IEN)/dysplasia was detected with equal frequency in the random biopsy and targeted biopsy groups. These new findings are also the basis for the recommendation for HDWLE with targeted biopsies, but without random biopsies, as a possible option for surveillance colonoscopy [206 - 210, 212].

\section{RECOMMENDATION 2.36}

The value of high-resolution virtual chromoendoscopy (NBI, FICE, iScan) in combination with targeted biopsies without random biopsies is not sufficiently defined and should therefore not be pursued as the sole strategy.

Evidence grade 1 , recommendation grade $B$, strong consensus

\section{Background}

Since the value of virtual chromoendoscopy (NBI, FICE, iScan) as an additional tool to increase the effectiveness of screening colonoscopy in ulcerative colitis is currently not clearly defined on the basis of the available studies, it should not be employed as a sole strategy [213-220].

\section{RECOMMENDATION 2.37}

In patients with suspected IEN/dysplasia, endoscopic monitoring should be carried out - if appropriate, after intensification of the anti-inflammatory therapy - within 3-6 months. Evidence grade 2, recommendation grade $B$, consensus

\section{RECOMMENDATION 2.38}

If there is evidence of an endoscopically nonresectable lesion with IEN/dysplasia or an adenocarcinoma, in view of the strong association with metachronous or synchronous carcinoma, a proctocolectomy should be performed.

Evidence grade 2, recommendation grade A, strong consensus

\section{RECOMMENDATION 2.39}

If polypoid lesions with dysplasia/IEN have been completely endoscopically resected and there is no evidence of dysplasia in the rest of the colon, colonoscopy at annual intervals can be recommended as a surveillance strategy.

Expert consensus, recommendation open, consensus

\section{RECOMMENDATION 2.40}

Following complete endoscopic resection of non-polypoid lesions with dysplasia/IEN without evidence of dysplasia in the rest of the colon, endoscopic surveillance should be performed annually.

Expert consensus, recommendation, consensus

\section{RECOMMENDATION 2.41}

If histologically confirmed IEN/dysplasia is detected by secondary diagnostic examination from endoscopically unremarkable areas, a new endoscopic examination should be performed by an examiner experienced in surveillance colonoscopy, if possible using chromoendoscopy with high resolution white light endoscopy (HDWLE).

Expert consensus, recommendation, consensus

\section{RECOMMENDATION 2.42}

If IEN/dysplasia is detected in endoscopically unremarkable areas, depending on the degree of dysplasia, endoscopic and bioptic surveillance should be carried out. If low-grade IEN is confirmed, endoscopic/bioptic surveillance should be repeated within 3-6 months.

Expert consensus, recommendation, consensus

Alternatively, proctocolectomy can be discussed with the patient. If high-grade IEN is confirmed, a recommendation for proctocolectomy should be given.

Expert consensus, open recommendation, consensus 
RECOMMENDATION 2.43

Polyps with dysplasia located proximal to the colon segments with anamnestically maximal macroscopic or histologic UC activity are regarded as sporadic adenomas and should be endoscopically resected if possible.

Expert consensus, recommendation, consensus

\section{Background}

The detection of intraepithelial neoplasia (IEN) and its classification grade are of critical importance when assessing the colon carcinoma risk in patients with ulcerative colitis. In untargeted quadrant biopsies, IEN was postoperatively identified in $74 \%$ of colectomy specimens with proven carcinoma, but also in $26 \%$ of colectomy specimens without proven carcinoma [221]. Results of a meta-analysis demonstrated that the carcinoma risk even in low-grade IEN is increased 9-fold [197].

In patients with ulcerative colitis and evidence of IEN, the prevalence of colon carcinoma is increased. A distinction is to be made between flat and polypoid lesions. If the colon is not in a predominantly inflammation-free state, it may be difficult to differentiate between inflammatory changes and IEN, since their appearance can be similar. Such lesions are generally classified as suspected IEN. In this case, the intensification of anti-inflammatory therapy with short-term endoscopic monitoring seems appropriate. On the other hand, evidence of flat, high-grade IEN found in endoscopically unremarkable areas bears a high risk of coincident carcinoma of between $42-45 \%$, and proctocolectomy should therefore be performed $[180,193]$.

While flat, low-grade IEN may be a precursor to high-grade IEN or carcinomas, low-grade IEN may equally be an indicator of synchronous carcinoma. A meta-analysis showed that patients in whom low-grade IEN is detected have a 9-fold increased risk of carcinoma development. However, there are a number of single studies which report that the risk for colitis-associated CRC development in patients with low-grade IEN is not, or is not significantly, increased [194, 197, 198]. A population-based study also found no increase in carcinoma development [196].

The presence of IEN, regardless of its grade, has been found to correlate with CRC development with a sensitivity and specificity of $74 \%$, while in the same study, it was shown that high-grade IEN is a less sensitive (34\%) but considerably more specific ( $98 \%$ ) indicator of CRC [221]. In a meta-analysis, low-grade IEN correlated with a 9-fold increased risk of CRC and a 12-fold increased risk of advanced neoplasia [197]. A recently-published systematic metaanalysis of cohort studies found low-grade IEN to be associated with an annual risk of $0.8 \%$ for incidence of CRC and $1.8 \%$ for incidence of advanced neoplasia. Factors significantly associated with progression of dysplasia were concomitant PSC (OR 3.4), invisible dysplasia (OR 1.9), distal localisation (OR 2.0) and multifocal dysplasia (OR 3.5). Furthermore, synchronous CRC was detected in $17 \%$ of patients who had undergone colectomy due to low-grade IEN [222]. Individual studies which found no increased risk of malignancy in patients with low-grade IEN should be considered with the meta-analyses in mind [196]. The diagno- sis of low-grade IEN is therefore associated with a substantial risk of carcinoma and has considerable prognostic implications [194, 198, 223].

Hence, the evidence concerning low-grade IEN is controversial and regular 3-6 monthly surveillance endoscopy with bioptic monitoring is considered a justifiable alternative to colectomy.

Elevated lesions with IEN were originally classified as dysplasiaassociated lesions or masses (DALM) [160]. The risk of CRC in the presence of DALM was estimated to be very high [193]. However, since the classification of DALM can present difficulties and showed inconsistencies, the term "DALM" was abandoned in the 2012 ECCO guidelines and replaced by the expression "raised lesions with dysplasia (RLD)" [189].

Such lesions may resemble sporadic adenomas, and can be endoscopically resected. If resection is histologically complete, and no IEN is detected either in the immediate vicinity or in the remainder of the colon, colectomy may not be necessary. Nevertheless, these patients have a tendency to develop raised lesions (RLD) and should therefore be closely monitored at short intervals [224-226]. A meta-analysis of 10 studies including 376 patients indicated there to be a low risk of developing colorectal carcinoma following polyp resection. However, since the risk of renewed dysplasia is increased approximately 10 -fold in these patients, frequent monitoring is required even after endoscopic resection [227].

Proctocolectomy is indicated in patients with non-resectable IEN/dysplasia or adenocarcinoma, since both are strongly associated with metachronous or synchronous carcinoma. In patients with completely resectable polypoid lesions with dysplasia/IEN, but without additional dysplasia in the remainder of the colon, screening colonoscopy at yearly intervals is recommended for surveillance [157]. The same approach is advisable in patients with completely resected non-polypoid lesions with dysplasia/IEN. If IEN/dysplasia is coincidentally detected in an endoscopically unremarkable area and confirmed by histological second opinion, a further monitoring endoscopy should be carried out, ideally by means of chromoendoscopy with high definition white light endoscopy (HDWLE), by an examiner experienced in surveillance endoscopy.

If low-grade intraepithelial neoplasia is confirmed in an endoscopically unremarkable area, endoscopic-bioptic monitoring should be performed within three to six months. Alternatively, the possibility of proctocolectomy may be considered. On the other hand, in patients with confirmed high-grade intraepithelial neoplasia found in endoscopically unremarkable areas, a recommendation for proctocolectomy should be given [157].

Polyps with dysplasia which occur proximal to the segments affected by colitis are regarded as sporadic adenomas and should be endoscopically resected if possible.

\section{RECOMMENDATION 2.44}

In patients additionally diagnosed with PSC, ursodeoxycholic acid can be given as a prophylaxis against colitis-associated carcinoma.

Evidence grade 2, recommendation grade 0 , consensus 


\section{Background}

Long-term therapy with 5-ASA should be offered to patients with ulcerative colitis as a prophylactic therapy for prevention of carcinoma (see also 3.18). In patients additionally diagnosed with PSC, ursodeoxycholic acid has been shown in a prospective, placebo-controlled follow-up study (PSC-UDCA study) to reduce the risk of colon carcinoma by $74 \%$ [228]. A cross-sectional analysis from a prospective surveillance study had previously indicated a considerable risk reduction [229]. On the other hand, in a prospective randomised study in 2009, a slightly higher overall mortality rate was found in patients with PSC who received a highdose ursodeoxycholic acid therapy $(28-30 \mathrm{mg} / \mathrm{kg}$ BW per day) [230]. On the basis of these data, treatment with ursodeoxycholic acid at a dose of $13-20 \mathrm{mg} / \mathrm{kg}$ BW per day is recommended.

\section{Treatment of active disease and remission maintenance therapy}

\section{General therapeutic goals}

In patients with ulcerative colitis, active disease flares are characterised by typical symptoms (bloody diarrhoea, tenesmus, imperative defaecation urgency). The therapeutic approach is determined by a combination of factors including not only the intensity of symptoms, but also the endoscopic localisation (proctitis, left-sided colitis, pancolitis), disease progression, response to previous therapy, disease duration, extraintestinal manifestations, concomitant illnesses and patient preferences. Individual treatment options should be discussed and agreed with the patient. Especially when weighing up outpatient vs. inpatient treatment, due consideration should be given to its practicability for both doctor and patient.

\section{STATEMENT 3.0}

The primary therapeutic aim for ulcerative colitis is to rapidly induce clinical remission and to maintain long-term steroidfree clinical and endoscopic remission.

Expert consensus, strong consensus

\section{RECOMMENDATION 3.1}

Anti-inflammatory therapy should be initiated only after objective evidence of inflammatory activity has been obtained. Expert consensus, recommendation, strong consensus

\section{Background}

All available anti-inflammatory therapies are aimed at influencing inflammatory activity, which thus represents the conditio sine qua non in the pharmacological treatment of ulcerative colitis. The severity of symptoms as described by the patient is often poorly correlated with the extent of objectively detectable inflammation as assessed by biomarkers, endoscopy or sonography, especially in disease of longer duration, where symptoms have been described to overlap with those of irritable bowel syndrome. Therefore, an increase in clinical activity is not necessarily caused by inflammation.

\section{RECOMMENDATION 3.2}

Following successful treatment of an acute disease flare, all patients should receive long-term remission maintenance therapy. Expert consensus, recommendation, strong consensus

\section{Background}

On the evidence of remission rates seen in the placebo groups of extensive studies, which reflect the spontaneous course of disease in patients with ulcerative colitis, most patients will relapse within 12 months of the previous flare. Therefore, the remission rate after 12 months is frequently $<50 \%$. Long-term therapy with mesalazine, thiopurines or biologics increases the likelihood of sustained remission [231 - 233]. The probability of prolonged remission is higher in patients who have attained deep clinical and endoscopic remission, as shown by retrospective analyses of data from the ACT-1 and ACT-2 trials in patients with ulcerative colitis receiving infliximab therapy [78, 234]. A positive correlation has been shown between deep remission as indicated by mucosal healing and the clinical course of disease $[78,79]$. Nevertheless, it has yet to be determined whether drug-induced mucosal healing in the sense of a "treat to target" strategy actually influences disease progression, or whether patients who achieve mucosal healing simply represent a subgroup of patients with a milder disease course.

\section{STATEMENT 3.3}

The choice and duration of the appropriate acute and remission maintenance therapy depends on the extent of disease, disease course (frequency and severity of flares), response to and side-effects of previous therapies, severity of the previous flare, the drug used to induce remission, the safety of the remission maintenance therapy, and the potential success of dysplasia and cancer surveillance.

Expert consensus, strong consensus

\section{RECOMMENDATION 3.4}

The risks and benefits of drug therapies should be weighed up against surgical options.

Expert consensus, recommendation, strong consensus 


\section{Therapy of uncomplicated ulcerative colitis}

Proctitis

\section{RECOMMENDATION 3.5}

Mild to moderately active proctitis should initially be treated with mesalazine suppositories at a dose of $\geq 1000 \mathrm{mg} / \mathrm{d}$ once daily.

Evidence grade 1, recommendation grade A, strong consensus

Mesalazine foam and mesalazine enema are considered equivalent therapeutic alternatives.

Evidence grade 2, strong consensus

\section{Background}

If ulcerative colitis activity is limited to the rectum, topical therapy with mesalazine is indicated. The effectiveness of topical mesalazine with regard to symptomatic, endoscopic and histologic response has been confirmed in numerous studies [235-237]. A dose of $1 \mathrm{~g} / \mathrm{d}$, at a minimum, should be administered. Higher doses have not been proven to bring additional benefit [238]. Suppositories have shown advantages in terms of drug release and patient tolerability, and should therefore be preferred [239]. While the additional administration of oral mesalazine can be considered [240], there are no specific data available on patients with proctitis. In a single study of patients with proctitis, rectal administration of mesalazine was found to be superior to oral therapy [241].

\section{RECOMMENDATION 3.6}

If monotherapy fails, rectal application of mesalazine should be combined with topical steroids or oral mesalazine-releasing preparations.

Evidence grade 1, recommendation grade $B$, strong consensus

\section{Background}

If response is lacking, rectal mesalazine should be combined with topical steroids. However, two meta-analyses demonstrated rectal mesalazine application to be more effective than topical steroids [242, 243]. A combination of beclomethasone dipropionate $(3 \mathrm{mg})$ and $2 \mathrm{~g}$ mesalazine enemas was found to be superior to either preparation alone [244]. Furthermore, results of a randomised trial demonstrated that $2 \mathrm{~g}$ budesonide rectal foam was more effective than placebo in patients with mild to moderate proctosigmoiditis [245], both alone and in combination with a mesalazine preparation [246]. A direct comparison with mesalazine is, however, lacking. For this disease localisation, intake of mesalazine granules appears beneficial [247].

Should the above outlined therapies remain unsuccessful, therapy adherence should be verified, the endoscopic severity confirmed and, if required, the therapeutic principles of more extensive severe ulcerative colitis applied. An additional therapeutic possibility is the rectal application of tacrolimus, which small trials have found to be efficacious [248, 249]. However, this therapy should be administered in centres with appropriate experience.

\section{Left-sided colitis}

\section{RECOMMENDATION 3.7}

Mild to moderate left-sided ulcerative colitis should initially be treated with rectal mesalazine in the form of enemas or foam $(\geq 1 \mathrm{~g} / \mathrm{d})$ in combination with oral mesalazine-releasing preparations ( $\geq 3 \mathrm{~g} / \mathrm{d}$ ).

Evidence grade 1 , recommendation grade $B$, strong consensus

\section{RECOMMENDATION 3.8}

Rectal application of mesalazine enemas or foams ( $\geq 1 \mathrm{~g} / \mathrm{d}$ ) should be preferred to topical steroid therapy. Evidence grade 2, recommendation grade A, strong consensus

\section{RECOMMENDATION 3.9}

Oral mesalazine-releasing preparations should preferably be administered in a single daily dose.

Evidence grade 2, recommendation grade $B$, strong consensus

\section{Background}

First-line therapy of mild to moderate left-sided ulcerative colitis should consist of a combination of oral and rectal mesalazine preparations, since combined therapy has been demonstrated not only to be more effective than oral therapy alone, but also to take effect more rapidly [250]. Both oral and rectal mesalazine applications have been shown to be more effective than placebo [235, $237,250]$. Therefore, if rectal application is not tolerated, oral therapy alone may be considered, the effectiveness of which has been confirmed in various studies. Topical application achieves a higher rectal active drug concentration [251]. Response rates for enemas or foams have not been found to differ significantly [252]. Oral mesalazine is not more effective than oral sulphasalazine, but it is associated with fewer side-effects [253]. There appears to be no significant difference in the effects of the various mesalazine preparations $[253,254]$. While the optimal daily dose may depend on the choice of preparation, it is important to ensure that the chosen dosage is sufficiently high ( $\geq 3 \mathrm{~g} / \mathrm{d}$ ) [253]. In left-sided colitis, the rectal application of beclomethasone dipropionate seems to be as effective as topical mesalazine, as a meta-analysis confirmed [255]. Rectally-administered budesonide foam has also been shown to be effective for the induction of remission [245]. Therapy adherence is an important consideration, especially with respect to mesalazine therapies, since a significant proportion of patients who fail to respond do so as a result of inadequate intake [256]. When prescribing, it should be kept in mind that mesalazine products are available in a range of different dosing options and with differing galenic characteristics (once daily dosing; tablets or pellets/granules). High-dose mesalazine once daily is not inferior to multiple daily doses $[257,258]$. The importance of topical applications should be discussed with the patient. 


\section{RECOMMENDATION 3.10}

If the symptoms of ulcerative colitis do not respond to the therapies discussed under 3.5-3.9, systemic steroid therapy should be initiated $(0.5-1 \mathrm{mg} / \mathrm{kg} B W / \mathrm{d}$ prednisolone equivalent). Evidence grade 1, recommendation grade A, strong consensus Budesonide MMX $9 \mathrm{mg} / \mathrm{d}$ should be administered to patients with mild to moderately active left-sided ulcerative colitis who show inadequate response, or are intolerant, to mesalazinereleasing preparations.

Evidence grade 1 , recommendation grade $B$, strong consensus

\section{Background}

The necessity for oral steroid therapy in patients with mild to moderately active ulcerative colitis depends not only on the clinical response to, and tolerance of, mesalazine therapies, but also on the wishes of the patient and the physician's judgment; there is no clear defining line. The median time until cessation of rectal bleeding at a dosage of $4.8 \mathrm{~g}$ mesalazine/d has been demonstrated to be 9 days, whereby stable remission was achieved only after a therapy duration of 37 - 45 days [259]. Therefore, oral steroid therapy should be initiated if clinical symptoms worsen during therapy or if bleeding persists for more than 14 days. While studies have shown budesonide MMX to be effective, oral budesonide without MMX galenic is ineffective in patients with ulcerative colitis [260]. In the Core I trial, the effectiveness of budesonide MMX was found to be equivalent to that of $2.4 \mathrm{~g}$ Asacol [261], while the Core II trial showed $9 \mathrm{mg}$ budesonide MMX to be significantly more effective than placebo [262]. A pooled analysis of the Core I and Core II studies concluded that intake of $9 \mathrm{mg}$ budesonide MMX was particularly beneficial for patients with mild or moderately active disease, and also for patients with left-sided colitis [263]. A further study showed the effectiveness of the additional administration of budesonide MMX in patients with inadequate response to mesalazine. In this situation, therefore, budesonide MMX may be considered as an additional therapy. A direct comparison with conventional steroids has not been performed. In the pivotal trials prior to drug approval, budesonide was found to have a good safety profile, with longterm safety data similar to those seen in the placebo groups.

\section{Extensive disease}

\section{RECOMMENDATION 3.11}

In patients with extensive disease, mild to moderate ulcerative colitis should initially be treated with an oral-release mesalazine preparation at a dose of $\geq 3 \mathrm{~g} / \mathrm{d}$ in combination with mesalazine enemas or foams.

Evidence grade 1, recommendation grade A, strong consensus

\section{RECOMMENDATION 3.12}

Systemic steroid therapy $(0.5-1 \mathrm{mg} / \mathrm{kg}$ BW/d prednisolone equivalent) should be initiated if symptoms of ulcerative colitis fail to respond to the therapies described in 3.5-3.9 and 3.11 or if a severe form of colitis is present at diagnosis. Evidence grade 1 , recommendation grade $A$, strong consensus

\section{Background}

The therapeutic principles in extensive, mild to moderately active ulcerative colitis essentially correspond to those applied in left-sided colitis; most studies include both patterns of involvement.

The superior effectiveness of a combination of oral and topical mesalazine therapy compared to oral monotherapy has been demonstrated in a randomised study [1]. As with left-sided colitis, sulphasalazine is equally efficacious, but associated with more side-effects [253]. Once-daily dosing has been found to be equally effective, independent of the formulation [254, 258]. It should be ensured that dosage is sufficiently high ( $\geq 3 \mathrm{~g} / \mathrm{d}$ ) [253].

In patients who show insufficient response to mesalazine maintenance therapy, steroid therapy should be initiated. There are no specific criteria with regard to disease severity, extent, or time to response. If the patient is already receiving immunosuppressive therapy, it is necessary to adapt steroid therapy depending on the medication history. The results of two studies indicate that in extensive colitis (unlike left-sided colitis), additional therapy with $9 \mathrm{mg}$ budesonide MMX was not superior to placebo [263].

\section{Remission maintenance in primarily uncomplicated ulcerative colitis}

\section{RECOMMENDATION 3.13}

Mesalazine should be applied as first-line maintenance therapy if the patient shows a response to either mesalazine or steroids. Evidence grade 1, recommendation grade A, strong consensus

\section{Background}

There is strong evidence supporting the use of mesalazine for maintenance of remission in ulcerative colitis. According to a recent meta-analysis of 42 studies and a total of 8928 patients, oral mesalazine is significantly more effective than placebo for maintenance of remission in terms of both clinical and endoscopic criteria [253]. Similar findings were demonstrated for rectal application of mesalazine in an additional meta-analysis, the remission rate after 12 months being significantly higher than in the placebo group [265]. Rectal mesalazine was shown to be particularly effective in distal ulcerative colitis. However, adherence to rectal therapy is very variable [266].

To date, there is no scientific evidence for a better therapy than mesalazine for maintenance of remission after response to either steroids or mesalazine [267]. Trials comparing mesalazine to thiopurines or biologics for maintenance of remission are lacking. Therefore, due to its more favourable side-effect profile, we recommend the administration of mesalazine as primary therapy in the situation described above. 
- Table 7 Mesalazine dosages proven to be effective in maintenance therapy.

\begin{tabular}{|l|l|l|}
\hline application & dose & comment \\
\hline oral monotherapy & mesalazine $\geq 2 \mathrm{~g} /$ day $[232]$ & $\begin{array}{l}\text { No clear dose-response relationship could be found in several studies; } \\
\text { once-daily intake preferable [258, 274, 276, 277, 279, 283] }\end{array}$ \\
\hline rectal monotherapy & No data available concerning the dose-response relationship \\
\hline oral-rectal combination therapy & $\begin{array}{l}\text { intermitt. rectal mesalazine of } 1-4 \mathrm{~g} \text { twice } \\
\text { weekly; } \\
\text { oral mesalazine daily } 1.6-3 \mathrm{~g} / \text { day }[284]\end{array}$ \\
\hline
\end{tabular}

Although its effectiveness has now been proven in three controlled studies, there are far fewer data for E. coli Nissle in comparison to mesalazine, as a result of which there is no consensus for its use as primary therapy. A meta-analysis of 4 randomised, controlled, blinded studies revealed no difference between mesalazine and E. coli Nissle for maintenance of remission [268]. Further studies are required to increase the pool of evidence and better define the position of E. coli Nissle in the therapeutic algorithm, especially in comparison to mesalazine [269].

\section{RECOMMENDATION 3.14}

The application route of mesalazine should be chosen according to the disease pattern. Proctitis and left-sided colitis should primarily be treated using rectal preparations.

Evidence grade 1, recommendation grade A, strong consensus

\section{Background}

Oral intake has been evaluated for all preparations. Conversely, the different rectal applications (suppositories, rectal foam, enema) have only been studied for mesalazine. In distal UC, rectal application of mesalazine has been shown to be superior not only to placebo [236, 265] but also to topical steroids [242]. The combination of oral and rectal therapy is more effective than oral monotherapy [270, 271]. In this combination, topical therapy can also be used intermittently [272]. Mesalazine formulations of the newer generation (e.g. multi-matrix formulations, granules), have a good release profile in the left-sided colon and are thus more effective in this situation than older-generation formulations with ileal release [247, 273, 274]. Hence, these new formulations represent an alternative in patients with distal colitis and poor therapy adherence.

\section{RECOMMENDATION 3.15}

A combination of oral and rectal mesalazine should be used as second-line maintenance therapy.

Evidence grade 1, recommendation grade A, strong consensus

\section{Background}

Two controlled studies have demonstrated the superiority of a combination therapy (oral and rectal mesalazine) compared with oral mesalazine alone in maintenance therapy [270, 271]. In patients who relapse in spite of oral or rectal mesalazine monotherapy, and whose acute episode is successfully treated with an oral-rectal combination therapy, the combination therapy should be continued for the maintenance of remission thereafter. In the two studies cited above, however, rectal therapy was applied only twice a week at a dose of $8 \mathrm{~g} \mathrm{[270]} \mathrm{and} 2 \mathrm{~g}$ [271], respectively. Thus, both dosage and application interval differ from the dosages currently recommended for induction of remission.

\section{RECOMMENDATION 3.16}

For maintenance therapy, mesalazine should be administered in dosages which have been demonstrated to be clinically effective ( $\vee$ Table 7$)$.

Evidence grade 1, recommendation grade A, strong consensus The medication should preferably be administered in a single daily dose.

Evidence grade 2, Recommendation grade A, strong consensus In view of its more favourable side-effect profile and comparable effectiveness, mesalazine should be preferred over sulphasalazine.

Evidence grade 1, recommendation grade $B$, strong consensus

\section{Background}

As yet, no clear dose-efficacy relationship has been established for maintenance therapy with mesalazine. In a recent meta-analysis, daily intake of $1.2 \mathrm{~g}$ mesalazine was statistically no less effective in sustaining remission after 12 months than a daily dose of $2.4 \mathrm{~g}$ [253]. However, patients taking the higher dose remained in remission for longer (median 175 days) than those receiving $1.2 \mathrm{~g}$ (median 129 days). This disparity was even more pronounced in the subanalysis of patients with extensive disease (median remission 143 days for $2.4 \mathrm{~g}$ vs. 47 days for $1.2 \mathrm{~g}$ ). However, after 12 months, the difference was no longer significant. While a 2011 meta-analysis showed better results for maintenance therapy with a daily dose of $\geq 2 \mathrm{~g}$ mesalazine compared with $<2 \mathrm{~g}$, the analysis included studies with a range of different mesalazine preparations (mesalazine, olsalazine, sulphasalazine 
and balsalazide) [232]. Likewise, a Cochrane analysis from 2016 identified a trend to better maintenance of remission under a daily dose of $\geq 2 \mathrm{~g} /$ day [253]. In conclusion, a mesalazine dose of $\geq 2 \mathrm{~g} /$ day should be administered for maintenance of remission. However, it remains unclear whether patients who required a higher dose to achieve remission, or who relapsed more frequently in the past, also require a higher dose to preserve remission. On the other hand, higher doses of mesalazine do not seem to be associated with increased side effects [275].

Once daily dosing has been shown in several studies to be as effective as multiple daily dosing for maintenance therapy [258, $274,276-279$ ] and should therefore be preferred in view of the improved therapy adherence. Regarding the rectal administration of mesalazine, there are no data to suggest a dose-response relationship. In most trials of rectal mesalazine (suppositories, foam) as monotherapy in adult patients, doses of $1 \mathrm{~g} /$ day were applied [280 - 282] and found to be superior to placebo. In the context of oral-rectal mesalazine combination therapy, rectal mesalazine has been demonstrated to be effective when administered twice weekly at a dose of both $4 \mathrm{~g}$ [270] and $1 \mathrm{~g}$, respectively [271].

A Cochrane analysis showed sulphasalazine to be more effective than other mesalazine preparations in maintenance therapy [253], whereby the difference was marginal (odds ratio 1.1; $95 \% \mathrm{Cl} 1.03$ 1.27). Moreover, no significant difference was found in the sideeffect profile of sulphasalazine compared with the other mesalazine preparations, which stands in contrast to clinical routine. Since most studies included patients already receiving sulphasalazine therapy, however, a selection bias cannot be excluded.

\section{RECOMMENDATION 3.17}

If effective, maintenance therapy with mesalazine should be continued for at least 2 years.

Evidence grade 2, recommendation grade $B$, strong consensus

\section{Background}

Few data exist concerning the value of long-term maintenance therapy in comparison to a simple surveillance approach. A controlled study of 112 patients revealed that in patients who have been in remission for one to two years, continued mesalazine therapy over 12 months can reduce the likelihood of relapse compared with placebo [285]. Patients in remission for longer than 2 years did not benefit from an additional 12 months' mesalazine therapy. However, for methodical and statistical reasons (necessary group sizes for full statistical power), the results of this analysis cannot be taken as a definitive indication of the value of mesalazine therapy for maintenance of remission in patients who have been in remission for over 2 years. Furthermore, the dosage used $(1.2 \mathrm{~g}$ mesalazine) is not in accordance with current guidelines.

In rare cases, mesalazine can lead to nephrotoxicity. Upon enquiry, 118 English and 45 international IBD centres reported 151 cases of diagnosed kidney damage, which occurred at an average of 3 years after therapy initiation. A genetic disposition was identified [286]. Although there is no evidence-based recommendation concerning the timepoint or subsequent intervals at which kidney parameters should be determined during longterm therapy, monitoring at 6- to 12-monthly intervals has been suggested [287].

It should be kept in mind that therapy adherence in patients receiving long-term mesalazine therapy is especially poor, at only around $50 \%$ [288].

\section{RECOMMENDATION 3.18}

Patients with ulcerative colitis should be offered long-term therapy with mesalazine from the perspective of carcinoma prevention.

Evidence grade 2, recommendation grade $B$, consensus

\section{Background}

A number of cohort and case-control studies have confirmed that both sulphasalazine and mesalazine are associated with a reduced risk of colorectal cancer in patients with ulcerative colitis [181, 289]. An analysis of risk factors for CRC in ulcerative colitis by Velayos [181] showed a statistically significant chemopreventive effect of mesalazine against the development of colorectal carcinoma. The chemopreventive effect of mesalazine in ulcerative colitis is not limited to high-risk patients [158, 192, 290]. The recommendation for long-term chemoprevention therefore applies to all ulcerative colitis patients except those with isolated proctitis [101, 158, 178, 190, 192, 290 - 298].

By suppressing mucosal inflammation, immunosuppressive drugs such as azathioprine can theoretically also exert an anticarcinogenic effect. The same applies to MTX and anti-TNF. However, the evidence is not conclusive $[178,181,190,229,291,299-$ 302], even though an observational cohort study of the CESAME group provides evidence that patients on long-term azathioprine therapy presented with a tendency towards a lower risk of colorectal carcinoma [303]. In conclusion, there is insufficient evidence to support a recommendation for thiopurines for chemoprevention in patients with ulcerative colitis.

\section{RECOMMENDATION 3.19}

In case of relapse, the therapy for maintenance of remission should be escalated.

Evidence grade 2, recommendation grade A, strong consensus

\section{RECOMMENDATION 3.20}

Options for a stepwise escalation of therapy for maintenance of remission are a dose escalation of oral/rectal combination therapy with mesalazine (Evidence grade 1, recommendation grade A), anti-TNF therapy (Evidence grade 1, recommendation grade A), therapy with vedolizumab (Evidence grade 1 , recommendation grade A) or therapy with thiopurines (Evidence grade 2 , recommendation grade $\mathrm{B}$ ).

Strong consensus 


\section{Background}

If relapse occurs while the patient is on maintenance therapy, options for therapy escalation should be considered. Although the range of drug therapies available to treat patients with ulcerative colitis has increased, and although certain drugs offer possibilities for combination therapies, there are still only a limited number of effective therapies. Therefore, the available therapies should be applied using the optimal dosage and at optimal dosing intervals. Alongside the dose escalation of existing therapy with mesalazine, therapies with thiopurines, TNF antibodies and vedolizumab including dose/interval adjustments offer additional options for escalation. Prior therapies, as well as any intolerances and comorbidities, should be considered when choosing the appropriate escalation strategy. Studies comparing immunosuppression with defined (optimised) basic therapies, or comparing the individual substance groups, are lacking, neither are there any formal escalation studies. In the absence of direct comparative studies, the most effective therapeutic strategy remains unclear. Further information concerning the use of thiopurines, TNF-antibodies and vedolizumab can be found in the chapter on maintenance of remission in ulcerative colitis with complicated disease progression (recommendations $3.33-3.34$ ).

\section{RECOMMENDATION 3.21}

Corticosteroids should not be used for maintenance therapy.

Evidence grade 1 , recommendation grade $A$, strong consensus

\section{Background}

There is no evidence to support the efficacy of either topical [304] or systemic corticosteroids [305] in maintenance therapy. Due to the hormonal character of steroids, severe adverse effects such as osteoporosis and cataract occur frequently during longterm therapy. In addition, corticosteroids as monotherapy - and in particular as part of a combination therapy - have been associated with a risk of severe infectious complications [306].

\section{Complicated forms of disease progression in ulcerative colitis/severe ulcerative colitis}

\section{RECOMMENDATION 3.22}

Patients with a severe acute flare of ulcerative colitis should be hospitalised for treatment.

Evidence grade 1, recommendation grade $B$, strong consensus Treatment should be administered in close cooperation with an interdisciplinary team including an experienced abdominal surgeon.

Evidence grade 3, recommendation grade B, strong consensus

\section{Background}

Severe ulcerative colitis can be defined using the well-established criteria of Truelove and Witts [83, 307]. The criteria include:
- Severe diarrhoea with 6 or more macroscopically bloody stools per day,

- Fever (with an average evening temperature of over $37.5^{\circ} \mathrm{C}$ or a temperature $>37.8^{\circ} \mathrm{C}$ on at least 2 out of 4 days),

- Tachycardia with a pulse $>90 / \mathrm{min}$, anaemia with a haemoglobin $<75 \%$ of the normal value and

- ESR $>30 \mathrm{~mm} / \mathrm{h}$.

These parameters define, in principle, systemic disease activity, which is usually also directly reflected in the patient's clinical condition. Such patients are severely ill. The use of scoring systems plays a mostly confirmatory role in clinical practice and is not mandatory. Generally, however, patients should be closely monitored for signs of systemic disease manifestation. Paediatric patients should be assessed using the PUCAl score [85]. Components of the PUCAI score are abdominal pain, rectal bleeding, stool consistency, stool frequency, nocturnal defaecation, and limitation of activity (total score between $0-85$ ). A PUCAI of $>65$ points indicates a severe colitis flare.

It is vitally important to make an accurate diagnosis at an early stage and to rule out intestinal infections [308]. Severe ulcerative colitis remains to this day a life-threatening condition, although the mortality rate lies by $<1 \%$ in specialised centres [84]. These patients therefore require hospitalisation. A meta-analysis investigating the response rate of patients with severe ulcerative colitis to corticosteroid therapy found a mean colectomy rate of $27 \%$ and a mortality rate of $1 \%$ [309]. In patients aged $>60$ years, hospitalisation is particularly important, since their mortality in this situation has been shown to be increased [310].

\section{Conventional therapy}

\section{RECOMMENDATION 3.23}

Patients with a severe flare of ulcerative colitis should be treated with systemic steroids (e. g. $1 \mathrm{mg} / \mathrm{kg}$ BW prednisolone equivalent per day).

Evidence grade 2, recommendation grade A, strong consensus

\section{RECOMMENDATION 3.24}

Patients with a severe acute flare should receive prophylactic treatment to prevent thrombosis.

Evidence grade 3, recommendation grade $B$, strong consensus

\section{RECOMMENDATION 3.25}

The response to systemic steroid therapy should be evaluated on the basis of clinical symptoms and objective parameters. Evidence grade 4, recommendation grade $B$, strong consensus 


\section{Background}

Since the middle of the last century, steroids have been the standard therapy in this situation [83, $311-315]$. Based on a number of studies, a dose of $1 \mathrm{mg}$ prednisolone/kg BW/day is recommended, whereas higher doses have not been shown to increase effectiveness [316]. A treatment duration of less than three weeks has been associated with an increased relapse rate. Furthermore, dosages below $15 \mathrm{mg}$ prednisolone/day are ineffective [311]. While, in principle, either oral or intravenous therapy can be administered, the latter should be favoured in patients with substantially disrupted motility. Response to steroid therapy should be assessed according to objective parameters including stool frequency, blood in the stool, haemoglobin level, ultrasound findings, endoscopic findings, CRP, blood count and faecal neutrophil markers.

In case of contraindications or intolerance to steroids, therapy with infliximab, ciclosporin or tacrolimus may be considered. Since the majority of studies of these substances have been carried out in steroid-refractory patients, these data will be more closely examined in that section.

In patients who do not respond to steroids, the possibility of therapy escalation or a switch of therapeutic strategy needs prompt consideration (after approximately three days). This is vitally important, since studies have demonstrated that treatment with non-effective drugs including steroids not only results in increased morbidity, but also in delayed surgery [317 - 320].

The required treatment goes far beyond pharmacological therapy and can only be guaranteed if the patient is hospitalised, since in addition to drug therapy, prior general and specific measures may be necessary. These include close monitoring of laboratory parameters, microbiological examinations (question regarding travel history), physio- and/or psychotherapeutic care and blood transfusions [321].

In addition, the following measures may be required:

1. parenteral fluid and electrolyte replacement. In particular, hypokalaemia and hypomagnesaemia should be avoided, since both conditions promote intestinal hypomotility and thus increase the risk of toxic megacolon [322].

2. discontinuation of all motility-inhibiting and otherwise potentially mucosa-damaging drugs, since these also increase the risk of toxic megacolon [6, 323-325].

3. sigmoidoscopy for confirmation of diagnosis and, at the same time, extraction of biopsies to rule out intestinal CMV-reactivation (together with the systemic CMV virus load). This is particularly important in patients with steroid-refractory disease; relevant CMV replication should be treated in this situation [326-331].

4. exclusion of Clostridium (C.) difficile infection, which occurs frequently in patients with severe ulcerative colitis and has been associated with increased mortality and morbidity [52, 310, 332 337]. Patients in whom Clostridium difficile is detected should be treated according to the DGVS guideline (see Chapter 4) [338]. It is not clear whether immunosuppressive therapy should be stopped in this case, since the data are conflicting [339, 340].

5. prophylactic administration of low molecular heparin to prevent thrombosis, since the risk of thrombosis is distinctly increased during an acute flare, regardless of other risk factors [337, $341-344]$.
6. initiation of nutrition therapy in patients with malnutrition. The enteral route should be favoured, as it is associated with fewer complications (9 versus 35\%) [345] and the parenteral route is not associated with better outcomes [346].

In children up to 16 years of age, the risk of hospitalisation due to a severe colitis flare is $30-40 \%$, and thus higher than in adults. Children and adolescents should be treated with methylprednisolone once daily at a dose of $1-2 \mathrm{mg} / \mathrm{kg}$ BW/d i. v. (maximum 40 $60 \mathrm{mg} / \mathrm{d}$ ) [309]. Approximately $30-40 \%$ of paediatric patients with severe ulcerative colitis fail to respond to the initial therapy and require therapy escalation with infliximab or ciclosporin. In patients with a PUCAI score of over 45 points on day 3 of i. v. steroid therapy, further diagnostic examinations (including sigmoidoscopy with exclusion of CMV colitis) should be performed and a therapy escalation prepared. If the PUCAI is $>65$ points on day 5 , therapy should be escalated, since these patients are unlikely to respond. As an alternative, colectomy should always be discussed with the parents and the patient. For the treatment of severe acute episodes in children and adolescents, separate guidelines have been issued by ECCO (the European Crohn's and Colitis Organization) and ESPGHAN (the European Society of Paediatric Gastroenterology, Hepatology and Nutrition) [347].

\section{Ulcerative colitis refractory to systemic steroid therapy}

\section{RECOMMENDATION 3.26}

Patients with moderate to severe ulcerative colitis who fail to respond adequately to systemic steroids, or who have contraindications or intolerances, should be treated with TNF antibodies (Evidence grade 2, recommendation grade B) or with ciclosporin $\mathrm{A}$ (Evidence grade 1 , recommendation grade $\mathrm{B}$ ) or tacrolimus (Evidence grade 2, recommendation grade B). Infliximab, if used, should preferably be given as combination therapy with a thiopurine (Evidence grade 2, recommendation grade B). Proctocolectomy should also be considered in therapeutic decisionmaking (Evidence grade 5, recommendation grade B).

Strong consensus

RECOMMENDATION 3.27

Patients with ulcerative colitis who have fulminant disease activity refractory to therapy with intravenous steroids should be treated with infliximab (preferably in combination with a thiopurine) (Evidence grade 2, recommendation grade B) or with ciclosporin A (Evidence grade 1, Recommendation grade B) or tacrolimus (Evidence grade 2, recommendation grade B). Proctocolectomy should also be considered (Evidence grade 5 , recommendation grade $B$ ).

Strong consensus 


\section{RECOMMENDATION 3.28}

In patients whose clinical condition deteriorates under the above-outlined (3.27) therapies, proctocolectomy should be performed (Evidence grade 4, recommendation grade B). Proctocolectomy may also be indicated if there is no clinical improvement after $4-7$ days.

Strong consensus

\section{Background}

If ulcerative colitis takes a steroid-refractory course, infectious causes such as $\mathrm{C}$. difficile or cytomegalovirus should be excluded (see Chapter 4). Intravenous steroid therapy has been shown to be more effective in confirmed ulcerative colitis refractory to oral steroids, e. g., in a retrospective study of 110 patients [348, 349]. However, almost half of the patients in this study went on to develop steroid-dependent disease. TNF antibodies have been clearly shown to be effective in the induction of steroid-free remission in patients with steroid-dependent disease who were taking steroids at study baseline (see comment on recommendation 3.29) [350 - 353]. In the same studies, the effectiveness of TNF antibodies was also shown in patients with steroid-refractory disease in relation to various endpoints. Although there were limits on steroid intake at study begin and the proportion of patients on steroid therapy was documented, some patients may not have been receiving optimally dosed steroids at baseline. Furthermore, it is not always possible to differentiate steroid-refractory from steroid-dependent disease and to analyse data from these groups separately. Various TNF antibodies known to be effective in UC can therefore be used in patients with a steroid-refractory disease course. In clinical practice, the choice of TNF antibodies in steroid-refractory disease largely depends on disease activity. In patients with less active disease, and therefore less need for fast improvement, a range of TNF antibodies including infliximab, adalimumab und golimumab can be applied, whereas only infliximab and calcineurin inhibitors have undergone controlled trials in patients with a fulminant disease manifestation. Since there are no uniform criteria for the differentiation of disease activity, this is ultimately subject to individual clinical estimation. In the last few years, patients with fulminant steroid-refractory disease have been the focus of numerous controlled studies, which will be discussed in detail below. However, assessing the response or non-response to therapy, and making a timely decision for or against a surgical approach, remains challenging. A number of factors can assist in clinical appraisal, the importance of which may be described as follows: In the clinical evaluation, a stool frequency of $>12$ /day after 2 days' therapy with intravenous steroids has been found to correlate with a proctocolectomy rate of $55 \%$ [354]. The Oxford Criteria, that have to be evaluated on day three, associate a stool frequency of $>8 /$ day, or $3-8 /$ day together with a CRP $>45 \mathrm{mg} / \mathrm{L}$, with a proctocolectomy rate of $85 \%$ during the hospital stay [355]. With regard to laboratory parameters, a prospective study of 67 patients showed that an ESR $>75 \mathrm{~mm} / \mathrm{h}$ or a body temperature of $>38^{\circ} \mathrm{C}$ at presentation is associated with a 5- to 9-fold increased risk of proctocolectomy [356]. Radiological appraisal should focus on data which are still relevant in contemporary clinical practice. These include a colon dilatation $>5.5 \mathrm{~cm}$ as measured in an abdominal scan, which is associated with a proctocolectomy rate of $75 \%$ [354]. In accordance with this, a retrospective study showed that evidence of ileus is associated with a proctocolectomy rate of $74 \%$ [357]. Data pertaining to endoscopic evaluation as a predictive marker for disease progression are limited to small case series [71, 358]. Since the most pronounced lesions in ulcerative colitis are located in the distal colon, evaluation by means of sigmoidoscopy is sufficient [359]. In everyday practice, it is important to assess the overall picture. Retrospective data show that deep ulceration, steroid refractory disease, colon dilatation and hypoalbuminaemia $(<30 \mathrm{~g} / \mathrm{dL})$ are major predictors (85\%) of the necessity for proctocolectomy [360].

Options for drug therapy include the following:

Antibiotics Two studies have addressed the question of whether additional antibiotic therapy should be given. In one of these studies, in which antibiotic therapy in 30 patients with ulcerative colitis was investigated in an open-label design, a twoweek therapy with amoxicillin, tetracycline and metronidazole appeared to be effective in steroid-refractory ulcerative colitis [361]. In contrast, an earlier randomised, placebo-controlled study of 39 patients with severe ulcerative colitis found no effect for metronidazole and tobramycin [362].

Ciclosporin The effectiveness of ciclosporin in this situation has been demonstrated in several studies. Historically, the first randomised and placebo-controlled trial, still using a dose of $4 \mathrm{mg} / \mathrm{kg}$ BW/day i. v., reported a rapid effect of ciclosporin A in the treatment of steroid-refractory ulcerative colitis [363]. While the small population size of 20 patients is a considerable limitation, 9 of 11 patients responded to ciclosporin therapy compared with 0 of 9 in the placebo group [363]. Later, the randomised, double-blinded study of d'Haens and colleagues sought to discover whether ciclosporin A can present an alternative to corticosteroid therapy. 30 patients with severe ulcerative colitis were treated with either $40 \mathrm{mg}$ methylprednisolone/day or $4 \mathrm{mg} / \mathrm{kg}$ BW/day ciclosporin A i. v.. After 8 days, treatment response was recorded in $8 / 15$ patients of the methylprednisolone group and in 9/14 patients of the ciclosporin A group [364]. A subsequent study compared $4 \mathrm{mg} / \mathrm{kg}$ BW/day with $2 \mathrm{mg} / \mathrm{kg}$ BW/day ciclosporin and found no difference in response on day 8 [365]. Although not all patients showed a steroid-refractory disease course at baseline, $2 \mathrm{mg} / \mathrm{kg}$ BW has now been established as the standard dose. In the study, serum trough levels were measured and the ciclosporin concentrations adjusted as appropriate. While optimal therapeutic serum trough levels have not been defined, levels between 250 and $400 \mathrm{ng} / \mathrm{mL}$ should be targeted, with dose adjustment based on trough level measurements. Both of the largest and most recent studies confirm these results. In the French study, 115 patients with steroid-refractory ulcerative colitis were randomised to receive ciclosporin ( $2 \mathrm{mg} / \mathrm{kg}$ BW) or infliximab [366]. Aim of the study was to demonstrate that infliximab is not inferior to ciclosporin, the primary endpoint being therapy failure at day seven. The data indicate that ciclosporin is not more effective than infliximab. Likewise, the CONSTRUCT study compared ciclosporin (2 mg/kg BW) with infliximab in 135 steroid-refractory 
patients and found no evidence of inferiority for infliximab [367]. The proctocolectomy rate in this study was $25 \%$ during hospitalisation, $30 \%$ within 3 months and $45 \%$ within the first year [367]. These current data are not included in the metaanalysis from 2005, making its conclusion that ciclosporin is not superior to the standard therapy, corticosteroids, practically untenable [368]. Nevertheless, the side effects associated with ciclosporin limit its use in everyday clinical practice.

The sum of the evidence from the various controlled and uncontrolled studies indicates that ciclosporin therapy can, at least in the short term, prevent proctocolectomy in $76-85 \%$ of patients [363 - 365, 369, 370]. In two studies including 76 and 142 patients treated with ciclosporin, 7-year proctocolectomy rates were $58 \%$ and $88 \%$, respectively $[371,372]$. Several analyses show that a transition from ciclosporin to thiopurine therapy significantly reduces the risk of subsequent proctocolectomy [370-373].

Tacrolimus Literature offering data on the use of tacrolimus is limited. A randomised, placebo-controlled trial compared two serum concentrations, $5-10 \mathrm{ng} / \mathrm{mL}$ and $10-15 \mathrm{ng} / \mathrm{mL}$, in steroid-refractory disease. While a dose-dependent efficacy was demonstrated, the study did not have the power to detect significant differences in patients with severe ulcerative colitis. Adverse events were particularly frequent in the high-dose group [374]. The second study, similarly, is a randomised, placebo-controlled trial over two weeks, which was able to demonstrate that oral tacrolimus is significantly more effective than placebo in patients with steroid-refractory ulcerative colitis [375]. A meta-analysis including both of these studies came to the same conclusion [376]. A long-term prognosis regarding surgery is difficult to deduce, owing to the relatively small patient numbers. The proctocolectomy-free rates after 1, 3, 6 and 12 months were $86 \%$, $84 \%, 78 \%$ and $69 \%$, respectively [376]. After 44 months, $57 \%$ of patients had not undergone proctocolectomy. There are a number of retrospective studies [377]. In a meta-analysis, significantly higher rates of clinical response were found for tacrolimus compared to placebo $(R R=4.61,95 \% \mathrm{Cl} 2.09-10.17, p=0.00015)$. Similar efficacy and safety data were reported from an open study of 100 patients with moderate to severe ulcerative colitis in which tacrolimus therapy was compared with an anti-TNF therapy [378]. On the basis of these data and experience from clinical practice, it can be concluded that tacrolimus presents a additional therapeutic alternative to steroid therapy.

Infliximab Two randomised, double-blinded, placebo-controlled studies (ACT 1 and 2) initially demonstrated the overall efficacy of infliximab for the treatment of ulcerative colitis [350]. In the ACT I trial, 364 patients with moderately active ulcerative colitis, who had previously not responded to corticosteroids and/ or immunomodulators, were treated. Sixty-nine per cent of patients treated with a dose of $5 \mathrm{mg} / \mathrm{kg}$ BW showed a clinical response in week 8 , compared to $61 \%$ of patients who received $10 \mathrm{mg} / \mathrm{kg}$ BW and $37 \%$ of patients in the placebo group. Rates of remission were $38.8 \%$ (5 mg/kg BW infliximab), $32 \%$ $(10 \mathrm{mg} / \mathrm{kg}$ BW infliximab) and $14.9 \%$ (placebo). Infliximab can thus be administered at a dose of $5 \mathrm{mg} / \mathrm{kg}$ BW at weeks 0,2 , and 6 . If effective, treatment should be continued at 8-weekly intervals thereafter as a maintenance therapy [350].
For the treatment of steroid-refractory disease, infliximab was evaluated in a double-blind, randomised pilot study by Järnerot and colleagues in 45 patients with severe ulcerative colitis who had previously failed to respond to conventional steroid therapy [379]. In seven patients in the infliximab group and 14 patients in the placebo group, proctocolectomy was necessary within 3 months after randomisation (statistically significant difference). The proctocolectomy rate in this cohort after three years was 12/ $24(50 \%)$ in the infliximab group and $16 / 21$ (76\%) in the placebo group [380]. In a retrospective multicentre study of 211 patients with steroid-refractory colitis treated with infliximab, the colectomy rates were $36 \%, 41 \%$ and $46 \%$ after 1,3 and 5 years, respectively [381]. The two largest controlled studies comparing infliximab with ciclosporin, the French trial and the CONSTRUCT study already cited above, indicated that infliximab is not inferior to ciclosporin [366, 367]. Colectomy rates reported in the CONSTRUCT study for patients treated with infliximab were $21 \%$ during the initial hospitalisation period, $29 \%$ after 3 months and $35 \%$ after 12 months [367].

So which factors can serve as predictors? Patients with steroidrefractory disease who present with markedly increased CRP, reduced serum albumin levels, seropositivity for perinuclear anti-neutrophil cytoplasmic antibodies (ANCAs) and severe endoscopic lesions have a high risk of imminent relapse or proctocolectomy [382, 383]. Maintenance of proctocolectomy-free remission is likely if there is a rapid response, mucosal healing and an infliximab serum trough level $>2.5 \mu \mathrm{g} / \mathrm{mL}$ in week 14 . Consistent with this, a low serum trough level in week $6(<2.5 \mu \mathrm{g} / \mathrm{mL})$ is an indicator of primary non-response [384]. What is the reason for low serum trough levels in patients with severe ulcerative colitis? It has been shown that infliximab is lost via the stool [385]. Although the induction schedule used in the CONSTRUCT study with dosing in weeks 0,2 and 6 has been adopted as standard, a small retrospective study of 50 patients provided evidence that accelerated induction may be associated with a reduced rate of early proctocolectomy [386]. In addition, thiopurine-naïve patients had better outcomes with regard to surgery [387].

\section{Weighing up infliximab versus calcineurin inhibitors}

The CYSIF study randomised 111 thiopurine-naïve patients with severe ulcerative colitis (Lichtiger Score $>10$ ) in spite of a prior five-day intravenous steroid therapy [366]. The patients were treated either with ciclosporin $(2 \mathrm{mg} / \mathrm{kg}$ BW for 8 days, target trough level $150-250 \mu \mathrm{g} / \mathrm{mL}$; followed by $4 \mathrm{mg} / \mathrm{kg}$ BW ciclosporin p. o.) or infliximab (5 mg/kg BW at weeks 0, 2 and 6) [366]. All patients showing a response on day 8 received oral azathioprine and began steroid tapering. The aim of the study was to demonstrate that ciclosporin therapy was associated with a lower rate of nonresponse. However, approximately $85 \%$ of the patients in both groups showed a response to therapy on day 7 . The treatment failure rate at day 98 was $60 \%$ in the ciclosporin group and $54 \%$ in the infliximab group (not significant). The proctocolectomy rate at day 98 was $18 \%$ in the ciclosporin group and $21 \%$ in the infliximab group (not significant). In addition, no differences were found regarding severe side-effects. Similarly, the aforementioned CONSTRUCT study, whose primary goal was to show that infliximab is 
not inferior to ciclosporin, failed to reveal any significant differences between the two therapies in terms of quality of life or rates of proctocolectomy, mortality or severe infections [367]. Consistent with this, a meta-analysis including six retrospective studies shows comparable remission rates for patients with severe, steroid-refractory ulcerative colitis treated with either ciclosporin or infliximab [388]. From an economic point of view, although infliximab patients had a significantly shorter hospital stay and incurred lower in-hospital costs, the total costs of therapy were higher [389].

Thus, there is no clear answer as to which of the substances should be used. This must be decided on an individual basis. In patients with low cholesterol and magnesium levels, ciclosporin is generally to be avoided due to the increased risk of neurological complications. In practice, the transition to maintenance therapy is easier in patients who respond to infliximab therapy. In the past, it has been argued that ciclosporin should be preferred because there are likely to be fewer complications in the event of surgery [390]. However, the evidence is based on a small case series and since, as yet, there are insufficient data on infliximab, no certain conclusion can be drawn. Much more important as regards the operational risk is the long-term use of steroids [391].

The next question is how to treat patients with steroid-refractory disease who fail to respond, or lose response to either infliximab or a calcineurin inhibitor. Is it then reasonable to try the other option? Most importantly, this is a situation in which surgical options should be discussed in detail and offered for consideration. Controlled studies for this situation are lacking, and the published case series are not helpful [392]. Therefore, third-line therapies should be administered, if at all, only in specialised centres, and in close consultation with the surgical partner.

\section{Complications}

Thromboembolic events occur more frequently during active disease, as already mentioned. Therefore, prophylactic anticoagulation is to be regarded as standard [341, 343]. Perforation during an acute flare, whether it occurs spontaneously or during endoscopic examination, is associated with a mortality rate of up to $50 \%$ [323]. A further complication is massive bleeding. When treating these seriously ill patients, it is critical not to miss the timepoint when conventional/drug therapy fails and surgical therapy (proctocolectomy) becomes necessary. Given the increasing number of therapeutic alternatives, the danger of delaying necessary surgical intervention is rather on the increase. Due to this dilemma, gastroenterologists/paediatric gastroenterologists and surgeons must cooperate closely, continually assessing together the patient's condition and response to therapy. Close patient monitoring is mandatory.

\section{Special situation: refractory proctitis}

Refractory proctitis is often clinically challenging. As a first step, differential diagnoses must be ruled out (HIV or chlamydia infection, irritable bowel syndrome, anatomic anomaly of the pelvic floor (prolapse), Crohn's disease, carcinoma). Secondly, the adherence of the patient to oral and topical drug therapies must be reaffirmed, and reduced bowel motility should be ruled out [393]. Therapy is then managed as described above for the classic refractory situation, even though hardly any studies have focused exclusively on proctitis. A few small studies support the topical application of calcineurin inhibitors (as enema or suppositories) [377, 394 -397]. Otherwise, the literature includes a number of uncontrolled studies which allow no general conclusions to be drawn. Critically, it should be noted that up to $10 \%$ of patients who undergo coloproctectomy due to refractory colitis suffer from distal colitis only.

\section{RECOMMENDATION 3.29}

In patients who respond to therapy with calcineurin inhibitors, azathioprine/mercaptopurine (Evidence grade 3, recommendation grade 0 ) or vedolizumab (Evidence grade 4, recommendation grade 0 ) therapy can be initiated. Patients who respond to therapy with TNF antibodies should continue to receive this therapy for maintenance of remission (Evidence grade 1 , recommendation grade $\mathrm{A}$ ).

Consensus

\section{Background}

In view of the high colectomy rate in patients receiving ciclosporin or tacrolimus due to steroid-refractory disease progression, all patients should receive remission maintenance therapy, including those who are mesalazine-naïve [363, 370, 398, 399]. Since, due to their frequent side-effects, calcineurin inhibitors should be discontinued after 6 months at the latest, other substances, such as thiopurines, should be used for maintenance of remission. Retrospective analyses have shown thiopurines to reduce the risk of colectomy after induction of remission with ciclosporin [372, 399]. An overlap of therapies is advisable, especially in thiopurine-naïve patients, such that thiopurines are introduced as soon as a response or remission has been achieved with the calcineurin inhibitor. After intravenous remission induction with ciclosporin, a bridging therapy with oral CsA can be administered until azathioprine takes therapeutic effect.

Lately, maintenance therapy with vedolizumab has been suggested as an alternative in patients who achieve remission with calcineurin inhibitors but have azathioprine-refractory disease [400]. Therapy for maintenance of remission with TNF antibodies following appropriate induction of remission is discussed at length under recommendation 3.34.

\section{Steroid-dependent ulcerative colitis}

\section{RECOMMENDATION 3.30}

Patients with steroid-dependent ulcerative colitis should be treated with a thiopurine (Evidence grade 2) or TNF antibodies (Evidence grade 1) (in the case of infliximab, possibly combined with a thiopurine (Evidence grade 2)), or with vedolizumab (Evidence grade 2).

Recommendation grade $B$, strong consensus 


\section{Background}

The efficacy of thiopurines, TNF antibodies and vedolizumab in steroid-dependent ulcerative colitis has been assessed in various studies. A general recommendation for the choice of an appropriate therapy for steroid-dependent disease cannot be given, due to the lack of comparative studies. The choice of drug is contingent on a number of factors including disease activity, the age and comorbidities of the patient, the potential side-effect profile, the clinical urgency for remission, the financial costs and the wishes of the patient. In steroid-dependent ulcerative colitis, azathioprine is significantly more effective than mesalazine for maintaining clinical and endoscopic remission. In an open-label study, 72 patients receiving steroid therapy with $40 \mathrm{mg} / \mathrm{d}$ prednisolone were randomised to receive additional therapy with azathioprine $2 \mathrm{mg} / \mathrm{kg} / \mathrm{d}$ or oral mesalazine $3.2 \mathrm{~g} / \mathrm{d}$ [401]. Of the patients in the azathioprine group, $53 \%$ attained steroid-free clinical and endoscopic remission after 6 months, compared with $21 \%$ of patients treated with mesalazine. In an open-label cohort study including 42 patients receiving azathioprine therapy, steroid-free remission rates of $55 \%, 52 \%$ and $45 \%$ were reported after 12, 23 and 36 months, respectively [402]. The evidence provided by these studies thus confirms the effectiveness of thiopurines in patients with steroiddependent ulcerative colitis.

Steroid-free remission was a secondary endpoint of the large clinical studies of anti-TNF. In none of these studies, however, was a clear differentiation made between steroid-dependent and steroid-refractory disease. The available data only show the proportion of patients treated with steroids during the therapy. Concerning infliximab, the ACT- 1 and ACT-2 trials investigated 364 patients with endoscopically-confirmed ulcerative colitis and moderate to severe disease activity despite treatment with corticosteroids and/or thiopurines (ACT-1) or with corticosteroids and/or thiopurines and/or mesalazine (ACT-2). All patients were anti-TNF-naïve and were randomised to receive either placebo or infliximab. At baseline, $56 \%$ of patients were being treated with corticosteroids, $38 \%$ at a dose of at least $20 \mathrm{mg} / \mathrm{d}$ prednisolone equivalent. Of those patients who took steroids at baseline, $21.5 \%$ achieved steroid-free remission under infliximab at week 30 , compared with $7.2 \%$ under placebo $(p=0.007)$ [350].

The ULTRA-2 study examined the effect of adalimumab in 494 patients with endoscopically-confirmed ulcerative colitis and moderate to severe disease activity despite therapy with corticosteroids and/or thiopurines. Patients were randomised to receive placebo or therapy with $160 \mathrm{mg}$ adalimumab at week $0,80 \mathrm{mg}$ at week 2, and $40 \mathrm{mg}$ at 2-weekly intervals thereafter [351]. Fiftynine per cent of patients were taking steroids at baseline, while $40 \%$ had previously failed anti-TNF-therapy. Thirty-one per cent of patients were steroid-free at week 16 , but only $16 \%$ of the placebo group $(p<0.05)$. At week $52,13.3 \%$ of patients receiving adalimumab therapy who had been taking steroids at baseline were in steroid-free remission, whereas this was true for only $5.7 \%$ under placebo $(p=0.035)$.

The effectiveness of golimumab in patients with moderate to severely active ulcerative colitis despite prior therapy with steroid or mesalazine or thiopurines was investigated in the PURSUIT trials [352, 353]. All study participants were anti-TNF-naïve. In these studies, the induction phase was separated from the main- tenance of remission phase. The clinical response rate at week 6 was $51.0 \%$ among patients treated with $200 \mathrm{mg}$ and then $100 \mathrm{mg}$ golimumab, compared with $54.9 \%$ in patients who had received $400 \mathrm{mg}$ and then $200 \mathrm{mg}$ [352]. In the PURSUIT-M study, 464 patients who responded to induction therapy were randomised again to receive remission maintenance therapy with golimumab or placebo. In the subgroup of patients taking steroids at baseline, $34.4 \%$ were in steroid-free remission under golimumab at week 54 , compared with $20.7 \%$ in the placebo group $(p=0.024)$ [353].

The above-described TNF antibodies are more effective than placebo in the induction and maintenance of steroid-free remission in patients receiving steroid therapy at baseline.

The usefulness of combination therapy with infliximab and azathioprine compared with infliximab or azathioprine alone was evaluated in the UC-SUCCESS study in biologic-naïve and mostly thiopurine-naïve patients [403]. In this randomised, controlled trial, a combination therapy with infliximab and azathioprine was found to be more effective than the monotherapies. At week 16, steroid-free remission was achieved by $39.7 \%$ of patients receiving combination therapy, compared with $22.1 \%$ in the infliximab group and $23.7 \%$ under azathioprine $(p=0.032)$.

Corresponding data on the effectiveness of adalimumab and golimumab as a combination therapy are not available. For these substances, the evidence indicates only that the production of autoantibodies is inhibited under co-immunosuppression. However, it remains to be shown whether this leads to improved treatment efficacy.

Steroid-free remission was also a secondary endpoint of the GEMINI-1 trial. In this study, which evaluated the effect of vedolizumab in patients with moderate to severe ulcerative colitis, 374 patients were enrolled in a randomised induction phase for treatment with vedolizumab or placebo. In an additional induction arm, 521 patients were enrolled in an open-label cohort. Responders at week 6 from both cohorts were then (re-)randomised to maintenance therapy with vedolizumab or placebo [404]. All study participants were refractory to steroids and/or to thiopurines or an anti-TNF therapy.

$53.7 \%$ of the patients in the GEMINI- 1 study were receiving steroid therapy at baseline, while $48 \%$ had failed anti-TNF therapy. Of those patients who took steroids at baseline and responded to induction therapy, $38.5 \%$ of patients randomised to receive vedolizumab maintenance therapy were in steroid-free remission at week 52 , compared with $13.9 \%$ of those receiving placebo $(p<0.001)$. The efficacy of vedolizumab therapy for both induction and maintenance was independent of concomitant therapy with steroids or immunosuppressive medications, and also independent of prior anti-TNF therapy.

Methotrexate (MTX) was recently the subject of a multicentre study of 111 patients with steroid-dependent ulcerative colitis [405]. The primary endpoint at week 16 was not reached, with $31.7 \%$ of patients in the MTX group compared with $19.6 \%$ of patients in the placebo group reaching steroid-free remission $(p=0.15)$. The secondary endpoint, the proportion of patients with steroid-free clinical remission at week 16 , was $41.7 \%$ in the MTX group and $23.5 \%$ in the placebo group $(p=0.04)$. Although the results showed a clear trend towards a therapeutic response, cur- 
rent data are not sufficient to issue a general recommendation concerning the use of methotrexate in patients with ulcerative colitis.

\section{Therapy of patients with ulcerative colitis and inadequate response to thiopurine therapy}

\section{RECOMMENDATION 3.31}

Patients with mild to moderate ulcerative colitis who fail to respond adequately to thiopurine therapy should be treated with TNF antibodies (Evidence grade 1) (in the case of infliximab, possibly in combination with thiopurines (Evidence grade 2)) or with vedolizumab (Evidence grade 2).

Recommendation grade $B$, strong consensus

\section{Background}

After infectious complications have been excluded, patients who fail immunosuppressive therapy should be treated with biologics, provided there are no relevant contraindications. In principle, a therapy with integrin antibodies can be administered even before TNF antibodies have been used. Infliximab, adalimumab, golimumab und vedolizumab have all been evaluated for the therapy of ulcerative colitis in patients refractory to thiopurines.

Three hundred and thirty-four/728 (46\%) of patients enrolled in the ACT-1 and ACT-2 studies had active disease in spite of immunosuppressive therapy [350]. Under infliximab, regardless of the dosage ( 5 or $10 \mathrm{mg}$ ), significantly more patients achieved clinical remission after 8 weeks compared to placebo. However, the response rate was not reported for the subgroup of patients who were refractory to immunosuppressive therapy. A Cochrane database review analysed 7 studies of infliximab therapy in patients with moderate to severe ulcerative colitis who were refractory to therapy with steroids or immunosuppression [406]. Three infusions (weeks 0,2 and 6) were more effective than placebo in inducing clinical remission at week 8 (RR 3.22, $95 \% \mathrm{Cl}$ $2.18-4.76)$. Again, no subgroup analysis was performed for patients refractory to immunosuppressive therapy.

In the ULTRA-1 study, adalimumab was reported to be superior to placebo in the induction of remission in patients with ulcerative colitis. One hundred and fifty-nine of 390 patients (39\%) were receiving immunosuppressive therapy at baseline $[407,408]$. At week 8 , in the subgroup of patients with concomitant immunosuppression, adalimumab induced remission in $15.1 \%(8 / 53)$, compared with $0 \%$ (0/15) under placebo. In patients receiving immunosuppression and steroid therapies at baseline, the rate of remission at week 8 was $12.2 \%$ (6/49) under adalimumab and $9.5 \%$ (2/34) under placebo. In the ULTRA-2 study, 173 of 494 patients were under immunosuppressive therapy [351]. Adalimumab $160 \mathrm{mg} / 80 \mathrm{mg} / 40 \mathrm{mg}$ every other week induced clinical remission in $8 / 53(15.1 \%)$ patients at week 8 , compared with $2 / 52$ (3.8\%) under placebo.

A prospective cohort study analysed 53 patients with moderate ulcerative colitis under infliximab or adalimumab therapy. The clinical response rate was $88.7 \%$, with no significant difference between the therapy groups [409]. All the enrolled patients were intolerant or refractory to an immunosuppressive therapy.
However, only 5/25 patients under adalimumab and 15/28 under infliximab were receiving treatment with immunosuppressants at baseline.

In the PURSUIT study with golimumab, $31.2 \%$ of patients with moderate to severe disease activity were treated with thiopurines. The concomitant immunosuppression had no influence on the outcome.

In patients who are refractory to immunosuppressive therapy, the question frequently arises as to whether immunosuppression should be continued during initiation of anti-TNF therapy. In the UC-SUCCESS trial, only patients with steroid refractory disease were enrolled. The patients either had to be naïve to immunosuppressants $(90 \%)$ or to have discontinued immunosuppressive therapy at least 3 months before baseline. Due to the high proportion of immunosuppressant-naïve patients, the data probably cannot be directly extrapolated to immunosuppressant-refractory patients. The recommendation for adjuvant immunosuppression in patients treated with infliximab is therefore based on indirect data indicating that concomitant immunosuppression can inhibit the production of autoantibodies and/or increase the effectiveness of therapy by raising trough levels [403, 410]. Neither subgroup analyses from clinical trials nor retrospective analyses of pharmacological samples have produced similar results for adalimumab or golimumab $[353,411]$. To date, there is no evidence that co-immunosuppression with adalimumab or golimumab enhances clinical efficacy.

In the GEMINI- 1 study, $17.8 \%$ of patients were enrolled while under immunosuppression and $16.6 \%$ under immunosuppression and steroids [404]. The subgroup analysis of response to induction therapy did not include all patients who had failed immunosuppressive therapy, but only those who had not also previously failed anti-TNF therapy. In this group, although a trend was observed towards an effect of vedolizumab in comparison to placebo, it did not reach significance ( $49 \%$ vs. $34.5 \%, p=0.08$ ). The same subgroup analysis in the maintenance phase, one year after re-randomisation of patients who responded to induction therapy, showed a significantly higher rate of remission under vedolizumab than under placebo $(44.6 \%$ under vedolizumab every 8 weeks, $50 \%$ under vedolizumab every 4 weeks and $18 \%$ under placebo). Concomitant immunosuppressive therapy did not significantly affect these results. The decision to treat with vedolizumab in this situation should be made according to UC disease activity, since a therapeutic response is not to be expected during the first few weeks of therapy.

Few data are available concerning the effectiveness of a second anti-TNF substance after failure of an anti-TNF therapy. Fourtyeight per cent of patients enrolled in the GEMINI-1 study of vedolizumab had previously failed anti-TNF therapy. Subanalyses revealed no significant differences between results from this subgroup and data from the total study population, suggesting that for vedolizumab, outcomes in patients with prior anti-TNF therapy failure are similar to those of anti-TNF-naïve patients.

In the OCTAVE-1 and OCTAVE-2 trials, which investigated the efficacy of tofacitinib in ulcerative colitis, patients with prior azathioprine therapy failure were included. Thus, tofacitinib could represent an option in patients with moderate to severe ulcerative colitis who are refractory to therapy with thiopurines. Since, at the 
time of going to press with the German version of the guideline, tofacitinib had not yet gained approval for ulcerative colitis therapy in Germany, this substance has not been included in the recommendations of these guidelines. Recommendations on the use of tofacitinib will follow in a forthcoming update of this guideline.

The use of tacrolimus in patients with ulcerative colitis is supported by several case series [248, 412]. However, there are no controlled clinical trials of this substance in patients with UC who have failed immunosuppressive therapy.

The benefits and risks of an immunosuppressive combination therapy, especially vis-à-vis the option of surgical therapy, should be critically discussed with the patient.

Ulcerative colitis with primary or secondary therapy failure under therapy with TNF antibodies

\section{RECOMMENDATION 3.32}

Patients with primary lack of response to therapy with TNF antibodies should be treated with vedolizumab (Evidence grade 2 , recommendation grade $B$ ) or calcineurin inhibitors (Evidence grade 3, recommendation grade B). Patients with moderate ulcerative colitis with secondary lack of response to therapy with TNF antibodies should be treated with alternative TNF antibodies (Evidence grade 4, recommendation grade B) or vedolizumab (Evidence grade 2, Recommendation grade $B$ ) or calcineurin inhibitors (Evidence grade 3, Recommendation grade B). Proctocolectomy should be considered. (Expert consensus, recommendation).

Strong consensus

\section{Background}

When considering treatment options available for patients who fail to respond to TNF antibody therapy, the differentiation between primary non-response and secondary treatment failure is clinically important. In principle, even in the case of primary treatment failure of a TNF antibody, the possibility of therapy intensification by dose increase should be considered. Especially in patients with endoscopically severe inflammation, the administered antibodies can be lost via the bowel ("faecal loss"), making a dose increase necessary [385]. In the case of primary lack of response to therapy, the choice of a substitute therapy depends to a large degree on the urgency of need for remission. Therefore, in patients with severe disease activity, calcineurin inhibitors are the therapy of choice, being most likely to induce rapid remission. Patients with mild inflammatory activity and less urgency for fast clinical improvement may alternatively be treated with vedolizumab. Switching from one TNF antibody to another TNF antibody is likely to be successful in only approximately $30 \%$ of patients with primary lack of response [413], and therefore cannot be recommended.

In patients with secondary treatment failure, the situation is different. In the ULTRA-2 study, the co-primary endpoint of clinical remission at week 16 in the subgroup of patients with prior anti-TNF treatment failure was not reached. The other clinical co-primary endpoint, clinical remission at week 52, was reached (10.2\% under adalimumab vs. $3.0 \%$ under placebo, $p=0.039$ ). The difference in this and other endpoints was smaller than in anti-TNF-naïve patients. The secondary endpoint of steroid-free remission at week 52 in patients receiving steroids at baseline was not reached in the subgroup of patients with prior anti-TNF failure.

In a meta-analysis, 8 studies focussing on the response to second-line TNF antibody therapy after secondary failure of a primary TNF antibody therapy were evaluated. All of the studies involved a switch from infliximab to adalimumab, with response rates varying between $23 \%$ and $92 \%$ and rates of remission varying between $0 \%$ and $50 \%$ [413]. Although the studies are very heterogeneous and do not allow a pooled data analysis, the current evidence base justifies a tentative switch from one antibody to another.

In the GEMINI-1 study, in which $48 \%$ of the patients had previously failed anti-TNF, the remission rate under vedolizumab was not influenced by prior therapy with TNF antibodies [404]. In a German cohort study of patients receiving vedolizumab, $25 \%$ of patients with ulcerative colitis or Crohn's disease were in clinical remission at week 14 [414]. As yet, no data have been published concerning the effectiveness of TNF antibodies after primary therapy failure with vedolizumab.

Two phase 3 studies investigated the efficacy of the JAK inhibitor tofacitinib (10 mg twice daily) as induction therapy in a total of 1139 patients with moderate to severe UC [415]. Patients enrolled in the studies had prior therapy failure with $>1$ of steroids, thiopurines or anti-TNF therapy (53-58\% of patients had previously received TNF antibody therapy). Remission at week 8 was reached by $18.5 \%$ and $16.6 \%$ under tofacitinib and $8.2 \%$ and $3.6 \%$ under placebo. Both differences were statistically significant. Under tofacitinib, increases in cholesterol and creatinine levels were observed, and in the $10 \mathrm{mg}$ group, an increased frequency of infections, especially herpes infections. At the time of going to press, tofacitinib did not yet gain approval in Germany for the treatment of patients with ulcerative colitis, and in view of the lack of clinical evidence, its placement within the therapy algorithm for ulcerative colitis cannot be conclusively evaluated.

Ozanimod, a modulator of the sphingosine-1-phosphate (S1P) receptor subtypes 1 and 5, has been investigated in a randomised phase 2 trial, in which $16 \%$ of the patients reached clinical remission at week 8 , compared with $6 \%$ under placebo $(p=0.048)$ [416]. Further studies are required to further assess the efficacy and safety of ozanimod.

Since patients who have experienced primary or secondary failure to TNF antibody therapy are generally very ill and frequently "beyond treatment" (i. e. drug therapy options have been exhausted), the option of proctocolectomy should always be considered and discussed with the patient.

\section{The use of biosimilars in patients with ulcerative colitis}

At the time of guideline preparation (in German language), only the infliximab biosimilar had been approved for the treatment of patients with ulcerative colitis. However, additional biosimilars will follow in the coming years. A general recommendation for the use of biosimilars will not be included in this guideline, since biosimilars are simply a different variation of a monoclonal anti- 
body (at the present time, infliximab), the use of which has already been discussed. However, since the use of biosimilars is currently the subject of considerable, and sometimes heated, debate, a few general comments concerning the use of biosimilars are included below.

The molecular structure of the currently available infliximab biosimilars is very similar to that of the reference product. All of the substances have shown similar physical and chemical characteristics, biological activity, pharmacokinetics and toxicity in animal studies and in healthy human subjects. Two phase 3 studies demonstrated a comparable efficacy, toxicity, and immunogenicity for biosimilar and reference product in patients with rheumatoid arthritis and ankylosing spondylitis [417, 418]. Several open-label studies indicate that the infliximab biosimilar is effective in patients with ulcerative colitis. On the basis of preclinical and clinical data, the use of infliximab biosimilars in rheumatoid arthritis, spondyloarthritis, ulcerative colitis and Crohn's disease has been approved by the EMA. It is to be expected that biosimilar substances will be increasingly introduced in the coming years, significantly expanding the spectrum of use of biosimilars in ulcerative colitis. The availability of biosimilars promotes competition and will contribute to cost reduction in financially overburdened health services. In addition, these important medications will be made available to a wider patient population. Thus, biosimilars seem to represent a real alternative to the original products. In spite of the lack of randomised studies in patients with UC, existing study data and clinical experience suggest that the efficacy of biosimilars is comparable to that of the original product, without any significant difference in the spectrum of side-effects. Negative data which would argue against the use of biosimilars in inflammatory bowel disease have not yet been published from studies, nor are they known from clinical practice. Biosimilars have been in use in a number of other indications for over 10 years, underlining that the introduction of new biosimilars can be considered safe, provided they have undergone testing in clinical studies.

Currently available data on patients switching from the originator to an infliximab biosimilar (e. g. from the NorSwitch trial [419]) do not reveal any relevant evidence of efficacy loss, an increased rate of side effects or problems concerning immunogenicity. However, since the evidence base is considerably less robust compared to the originators, further study data should be collected to dispel any possible concerns in this respect. Gastroenterologists should familiarise themselves with the biosimilar concept in the therapy of inflammatory bowel disease and address unfounded concerns relating to the safety and efficacy of these substances. Regardless of the relative safety of infliximab biosimilar use to date as a primary therapy and presumably also in the case of a switch, the production process of biosimilars must be subject to the highest quality control requirements. Furthermore, the greatest possible transparency must be demanded with regard to the manufacturing process and the prescription of biologic substances. In principle, the use of biologics or biosimilars should be bound by a requirement that the attending physician can clearly distinguish the products he or she prescribes, and that he or she retains the decision-making authority, at least with regard to switching therapy between the original product and a biosimilar during ongoing therapy. Repeated switching from biosimilar to originator, or between different biosimilars ("multiswitch") cannot be recommended, since no data are available.

\section{Maintenance therapy of remission in patients with complicated disease progression in ulcerative colitis}

\section{RECOMMENDATION 3.33}

After achieving remission, maintenance therapy with thiopurines should be used in patients with mild to moderate disease activity if early or frequent relapses occur under optimallydosed regimens with mesalazine or if the patient is intolerant to mesalazines (Evidence grade 1), or if there is steroiddependent disease progression (Evidence grade 2 ) or if the patient has responded to induction therapy with ciclosporin or tacrolimus (Evidence grade 3 ).

Recommendation grade $B$, strong consensus

\section{Background}

Several randomised, controlled studies have investigated the effectiveness of thiopurines (azathioprine and mercaptopurine) for maintenance of remission in patients with ulcerative colitis [401, 420-425]. A Cochrane meta-analysis of 7 of these studies, including a total of 302 patients, concludes that the overall effectiveness of thiopurines is moderate and that evidence supporting the use of thiopurines in ulcerative colitis is significantly weaker than in Crohn's disease [233]. The quality of older trials is generally not comparable to the results of contemporary studies. It is also unclear on the basis of these studies whether concomitant medication with mesalazine enables therapy optimisation. Notable is the high rate of azathioprine-induced side effects, especially the onset of acute pancreatitis, hepatopathy and bone marrow suppression. Additional evidence for the use of thiopurines in ulcerative colitis can be found in retrospective studies [426431]. A study from Oxford with 346 patients showed a remission rate of $58 \%$ under azathioprine, increasing to $87 \%$ in patients treated with azathioprine for more than 6 months. Assuming a strict definition of disease relapse, the remission rate after 5 years was $62 \%$, and $81 \%$ if mild flares could be treated with short-term steroid therapy. The median time to relapse after discontinuation of azathioprine treatment was 18 months [427]. In a more recent retrospective study, approximately one-third of patients relapsed within 3 years of stopping thiopurine therapy, whereby patients with extensive ulcerative colitis, in particular, showed biological signs of increased inflammatory activity at treatment discontinuation, or only endured thiopurine therapy for a short period of time [432].

Calcineurin inhibitors (ciclosporin A, tacrolimus) are used as rescue therapies in patients with steroid-refractory ulcerative colitis. Because of their side-effect profile, they should, if possible, be discontinued within 6 months. Therefore, calcineurin inhibitors play an important role as bridging therapy until IBD therapies with delayed-onset efficacy can take effect. In this case, thiopurine treatment should be given as an overlap therapy as soon as the patient shows a clear response to calcineurin inhibitors. At the same time, steroids should be tapered. By combining thiopur- 
- Table 8 Overview of remission rates under TNF-antibody therapies.

\begin{tabular}{|c|c|c|c|}
\hline TNF-antibody & study & timepoint & remission rate \\
\hline \multirow[t]{2}{*}{ infliximab } & ACT-1 [350] & week 54 & $\begin{array}{l}35 \% \text { IFX } 5 \mathrm{mg} / \mathrm{kg} \\
34 \% \text { IFX } 10 \mathrm{mg} / \mathrm{kg} \\
17 \% \text { placebo }\end{array}$ \\
\hline & ACT-1 [350] & week 30 & $\begin{array}{l}26 \% \text { IFX } 5 \mathrm{mg} / \mathrm{kg} \\
36 \% \text { IFX } 10 \mathrm{mg} / \mathrm{kg} \\
11 \% \text { placebo }\end{array}$ \\
\hline \multirow[t]{3}{*}{ adalimumab } & ULTRA-2 [351] & week 8 & $\begin{array}{l}16.5 \% \text { adalimumab } \\
9.3 \% \text { placebo }\end{array}$ \\
\hline & & week 52 & $\begin{array}{l}17.3 \% \text { adalimumab } \\
8.5 \% \text { placebo }\end{array}$ \\
\hline & Pooled data: ULTRA-1,-2,-3 [436] & week 208 & $24.7 \%$ adalimumab \\
\hline golimumab & PURSUIT-M [353] & week 54 & $\begin{array}{l}27.8 \% \text { (clinical remission) and } 42.4 \% \text { (mucosal healing) under } \\
\text { golimumab } 100 \mathrm{mg} \text { s. c. every } 4 \text { weeks; } \\
\text { placebo: } 15.6 \% \text { clinical remission and } 26.6 \% \text { mucosal healing }\end{array}$ \\
\hline
\end{tabular}

IFX: infliximab.

ines with calcineurin inhibitors, the high rate of colectomy associated with calcineurin inhibitor monotherapy, which lies between $36 \%$ and $69 \%$ at 12 months, can be significantly reduced [369, $370,398,399]$. Retrospective case series have shown that by using thiopurines, the risk of colectomy after therapy with calcineurin inhibitors can be reduced [369, 370, 433]. In view of the delayed-onset effect of thiopurines, calcineurin inhibitors should be discontinued 3-6 months after beginning overlap therapy. The optimal dose of azathioprine in patients with ulcerative colitis has not yet been identified in studies. Therefore, in analogy to Crohn's disease, the usual dosage for azathioprine in ulcerative colitis is $1.5-2.5 \mathrm{mg} / \mathrm{kg} \mathrm{BW} /$ day or half of this dose for 6-mercaptopurine [242]. Due to the pharmacodynamics of the substance (onset of therapeutic effect only after $6-12$ weeks), longer-term therapy planning is required.

\section{RECOMMENDATION 3.34}

In patients who respond to induction therapy with TNF antibodies, maintenance therapy with TNF antibodies with or without thiopurines is recommended.

Evidence grade 1, recommendation grade A, strong consensus In patients who respond to induction therapy with vedolizumab, maintenance therapy with vedolizumab is recommended. Evidence grade 1, recommendation grade A, strong consensus

\section{Background}

Maintenance of remission with TNF antibodies

In the ACT studies, it was demonstrated that patients receiving infliximab therapy were significantly more likely to remain in remission at weeks 30 and 54 (ACT-1 only) than patients under placebo (see Table 8). Notably, however, the proportion of patients with steroid-free remission, although statistically signifi- cant, was small. In ACT-1, rates of steroid-free remission at week 54 were $24 \%$ (infliximab $5 \mathrm{mg} / \mathrm{kg}$ ), $19 \%$ (infliximab $10 \mathrm{mg} / \mathrm{kg}$ ) and $10 \%$ (placebo). In ACT-2 the rates at week 30 were $18 \%$ (infliximab $5 \mathrm{mg} / \mathrm{kg}$ ), $27 \%$ (infliximab $10 \mathrm{mg} / \mathrm{kg}$ ) and $3 \%$ (placebo) [350].

One study reported the long-term outcomes of 121 patients with refractory ulcerative colitis who were treated with infliximab [434]. Of the $67 \%$ of patients who responded to infliximab therapy, $68 \%$ showed a lasting response (median observation period 33.0 months; $17.0-49.8$ months). A total of $17 \%$ underwent colectomy.

Adalimumab was also shown to be superior to placebo for maintenance therapy. Remission rates in the ULTRA-2 study at week 8 were $16.5 \%$ (adalimumab) and 9.3\% (placebo, p=0.019) and at week 52, $17.3 \%$ (adalimumab) vs. $8.5 \%$ (placebo, $p=0.004)$ [351]. Since infliximab was already approved at the time of the ULTRA-2 study, a relevant proportion (40\%) of patients had previously been treated with infliximab. For these patients, in terms of remission at week 8 , there was no difference to placebo; at week 52 , only $10.2 \%$ of these patients were in remission (placebo $3 \%, p=0.039$ ). In 2015, a review of published case series reported remission rates of $0-50 \%$ in patients previously treated with infliximab $[413,435]$. In addition, longterm adalimumab therapy over a duration of 52 weeks led to a reduced hospitalisation rate [435]. Data on the use of adalimumab beyond week 52 are provided by the pooled analysis of ULTRA-1, ULTRA-2 and ULTRA-3 [436]. For 199 patients, four years of therapy data are available; the remission rate at week 208 was $24.7 \%$, and approximately $60 \%$ of patients who were in remission after one year remained in remission at week 156.

In the PURSUIT-M trial, the efficacy of subcutaneous (s.c.) golimumab was demonstrated in patients with ulcerative colitis who had received no prior anti-TNF therapy [353]. In patients who responded to golimumab at week 6 , this response was sus- 
tained at week 54 in $47 \%$ (50 mg golimumab s. c. every 4 weeks) and $49.7 \%$ (100 mg golimumab s. c. every 4 weeks) of these patients, compared to $31.2 \%$ on placebo. In patients receiving $100 \mathrm{mg}$ golimumab s. c. every 4 weeks, clinical remission in $27.8 \%$ and mucosal healing in $42.4 \%$ at week 54 were reported (placebo $15.6 \%$ and $26.6 \%$, respectively).

Maintenance of remission with vedolizumab

Data concerning the effectiveness of long-term vedolizumab therapy is provided by the GEMINI-I study [404]. At week 52, a remission rate of $41.8 \%$ was reported for 8 -weekly treatment with $300 \mathrm{mg}$ vedolizumab, and $44.8 \%$ for 4 -weekly treatment (placebo $15.9 \%$ ). However, the difference between response to 4- and 8-weekly dosing was not significant [437]. In a subgroup analysis of the GEMINI-1 trial data, vedolizumab was found to be effective in both anti-TNF-naïve and anti-TNF-exposed patients, albeit with lower remission rates among the patients with prior anti-TNF therapy failure (46.9\% versus $36.1 \%$ in week 52) [438]. In a recently published follow-up study, even three years after successful induction therapy, $50 \%$ of patients who had initially responded to vedolizumab therapy were reported to have mucosal healing [439]. Real-world data from a German cohort document steroid-free clinical remission after 54 weeks in $22 \%$ of patients initially treated with vedolizumab [440]. In $56 \%$ of these patients, vedolizumab was discontinued after a median of 18 weeks. In a French cohort, $40.5 \%$ of patients were in steroidfree clinical remission under vedolizumab after one year. The majority of patients who were in remission at week 22 maintained this remission until week 54 [441]. A meta-analysis confirmed the usefulness of vedolizumab in maintenance therapy, demonstrating its effect in terms of relapse prevention [442].

Combination therapies in maintenance therapy

In the UC-SUCCESS trial, the efficacy of azathioprine $(2.5 \mathrm{mg} /$ $\mathrm{kg}$ daily dose) and infliximab (5 mg/kg at weeks $0,2,6,14)$ as combination therapy was examined with regard to remission at week 16 [403]. The combination therapy, with a steroid-free remission rate of $39.7 \%$ in week 16 , was found to be superior to either of the substances as monotherapy (infliximab monotherapy $22.1 \%$; azathioprine monotherapy $23.7 \%$ ). Mucosal healing occurred in $62.8 \%$ of patients receiving combination therapy, compared to $54.6 \%$ under IFX monotherapy and $36.8 \%$ under azathioprine monotherapy. It should be noted, however, that the remission rate was examined only at the week 16 timepoint and not over a longer period. Furthermore, only patients naïve to therapy with thiopurines and TNF antibodies were enrolled.

Robust data on the use of a combination therapy consisting of azathioprine together with adalimumab or golimumab in the therapy of patients with ulcerative colitis are not available. In a retrospective analysis of 23 patients treated with immunomodulators (thiopurines $n=14$; MTX $n=9$ ) after losing response to adalimumab therapy, 11 of these patients ( $48 \%$ ) showed a reduction in adalimumab antibodies, a recovery of adalimumab trough levels, and a clinical response [443]. However, since only two of the 23 patients had ulcerative colitis, the results do not allow for a general recommendation on combination therapy with adalimumab in ulcerative colitis. Prospective data in patients with Crohn's disease indicate that outcomes are no better than under adalimumab monotherapy, although the combination therapy showed advantages with respect to the secondary endpoint of mucosal healing [444].

Studies offering head-to-head comparisons of the individual substances regarding their efficacy in remission maintenance are lacking. Therefore, it is not possible to give a recommendation as to which substance should be preferred. Usually, however, the substance used to induce remission is also administered for maintenance therapy.

\section{RECOMMENDATION 3.35}

Methotrexate, ciclosporin and tacrolimus should be used for maintenance therapy only in exceptional cases.

Evidence grade 4, recommendation grade $B$, strong consensus

\section{Background}

To date, there is insufficient evidence in support of the use of methotrexate or tacrolimus for remission maintenance. A randomised, placebo-controlled trial of methotrexate in 67 patients showed that oral treatment with $12.5 \mathrm{mg}$ methotrexate per week failed to maintain remission over a duration of 9 months [445]. Various retrospective studies have examined the efficacy of methotrexate for maintenance therapy in patients with ulcerative colitis. Most patients had previously failed therapy with, or shown intolerance to, azathioprine. The response/remission rates reported in these studies were between $30 \%$ and $80 \%[424,446]$. Overall, the data on maintenance therapy in patients with ulcerative colitis are very heterogeneous. A systematic Cochrane metaanalysis concluded that methotrexate cannot currently be recommended for maintenance therapy [447].

\section{RECOMMENDATION 3.36}

Due to the lack of evidence, no recommendation can be made on the duration of maintenance therapy with thiopurines, TNF antibodies and vedolizumab. However, long-term continuation of maintenance therapy will frequently be necessary. Expert consensus, strong consensus

\section{Background}

For all the above-named substances, the existing data are insufficient to assess either the optimal duration of therapy or the optimal premises for therapy discontinuation.

The effectiveness of azathioprine was investigated in a retrospective study in 346 patients with ulcerative colitis. The remission rates after one, two and five years were $95 \%, 69 \%$ and $55 \%$, respectively [427]. However, the retrospective character of the study must be considered a limitation. Moreover, remission and relapse of ulcerative colitis were defined only by the absence or use of steroid medication.

Likewise, the optimal therapy duration for biologics (TNF antibodies, vedolizumab) is unknown. In the respective pivotal studies, during which patients received maintenance therapy for approximately one year, superiority in maintenance therapy com- 
pared to placebo was documented for infliximab [350, 351, 353] and vedolizumab [404]. Anti-TNF therapy should therefore be continued for at least one year. The extent to which patients who are in remission after one year benefit from continuation of therapy, and whether remission should be defined purely clinically or endoscopically, has yet to be determined.

A considerable proportion of patients will experience a relapse within 12 months of discontinuing anti-TNF therapy. In a meta-analysis from 2016, a relapse rate of $28 \%$ was found in patients treated with infliximab or adalimumab 12 months after therapy discontinuation; $80 \%$ responded to the same therapy upon reintroduction [80]. A further meta-analysis including patients with both Crohn's disease and ulcerative colitis determined a relapse rate of approximately $50 \%$, two years after discontinuation of anti-TNF therapy [448].

\section{RECOMMENDATION 3.37}

The apathogenic Escherichia coli strain Nissle 1917 can be used in justified cases as an alternative to mesalazine.

Evidence grade 2, recommendation grade 0 , strong consensus

\section{Background}

A 2015 meta-analysis evaluated a total of six studies investigating the use of E. coli Nissle (ECN) 1917 in ulcerative colitis. It concluded that EcN was as effective for maintenance therapy as mesalazine [268]. It should be noted, however, that in some of the included studies, EcN was tested only against placebo, and it showed no significant advantage over placebo during induction therapy. Furthermore, all studies taking mesalazine as comparator took a daily dose of $500-800 \mathrm{mg}$, a dosage well below that regarded as effective (see recommendation 3.13). A large proportion of consensus participants considered the studies to be insufficiently valid.

\section{RECOMMENDATION 3.38}

Therapeutic drug monitoring can be carried out during therapy with thiopurines, TNF antibodies and vedolizumab, if required to support clinical decision-making.

Expert consensus, recommendation open, strong consensus

Under therapy with calcineurin inhibitors, trough levels should be monitored regularly.

Expert consensus, strong recommendation, strong consensus

\section{Background}

Despite the increasing number of drug therapies available for ulcerative colitis and the possibility of combination therapies with certain drugs, the number of effective therapies remains limited. Moreover, since the response and remission rates under second and third-line TNF antibody therapies are very poor, the available therapies should be administered at an optimal dose and at optimal dose intervals.

Drug monitoring of TNF antibodies
The serum trough levels of infliximab, adalimumab and golimumab correlate with their clinical effectiveness, and high trough levels are more often found in patients with clinical and endoscopic remission in the form of mucosal healing [352, 449-453]. In a recent meta-analysis of 22 studies including a total of 3483 patients with Crohn's disease or ulcerative colitis, infliximab trough levels $>2 \mu \mathrm{g} / \mathrm{ml}$ correlated with a good clinical response and lower CRP levels [454]. For adalimumab, trough levels of $>6 \mu \mathrm{g} / \mathrm{L}$, and for golimumab, trough levels of $>2.5 \mu \mathrm{g} / \mathrm{mL}$ at week 6 and $>1.4 \mu \mathrm{g} / \mathrm{mL}$ in maintenance therapy, have been reported to be associated with a high likelihood of clinical remission [456]. In patients who developed detectable antibodies directed against biologics and, at the same time, had low trough levels, treatment continuation was successful in only $17 \%$ of IBD patients, whereas switching to a different TNF antibody achieved a response in approximately $92 \%$ [457]. These figures, however, originate from a retrospective data analysis [457]. Furthermore, it must be taken into account that a considerable proportion of patients who switch to adalimumab after failure of infliximab therapy will develop anti-adalimumab antibodies, which lead to a loss of response [458]. If, despite low trough levels and detectable antibodies to an anti-TNF agent, the dose is increased or the application interval shortened, an increased rate of allergic reactions is to be expected; a meta-analysis from 2014 showed patients with anti-infliximab antibodies to have a 2.4-fold increased risk of allergic infusion reaction and a 5.8-fold risk of severe allergic reaction [459]. If, however, autoantibodies to TNF antibodies are detected, but at the same time, trough levels are high, it should be noted that such antibodies are frequently transient [460] and disappear in two out of three patients during the course of treatment [461]. A recent study of 247 patients with IBD showed that therapeutic drug monitoring, i. e. measurement of trough levels and corresponding autoantibodies, resulted in therapeutic consequences in over $70 \%$ of patients [462]. Nevertheless, therapeutic drug monitoring cannot answer all clinical questions and, due to its numerous limitations, can currently be recommended only as one of many possible tools for therapy management in patients with ulcerative colitis.

Drug monitoring of vedolizumab

In the pivotal study GEMINI-I, it was reported that a higher trough level at week 6 was associated with higher response and remission rates [404]. Although clinical experience of trough level determination for vedolizumab is still very limited, there are indications that drug monitoring can play a useful role in optimising vedolizumab therapy. A clear recommendation in favour of vedolizumab drug monitoring cannot be given at the present time [463 - 465].

Drug monitoring of calcineurin inhibitors

The effective trough levels of calcineurin inhibitors are subject to considerable interindividual variation. Therapy management therefore necessitates regular trough level determination and dose adjustment. The effectiveness of ciclosporin and tacrolimus in patients with ulcerative colitis has already been addressed in detail (see background to recommendations 3.26-3.28). In summary, an initial dose of $2 \mathrm{mg} / \mathrm{kg}$ BW ciclosporin A is currently recommended, with subsequent dose adjustment according to trough levels. While the optimal level remains unclear, the target trough level should be $250-400 \mathrm{ng} / \mathrm{mL}$. Tacrolimus is initially administered at a dose of $0.05 \mathrm{mg} / \mathrm{kg}$ BW orally twice daily and the dose thereafter modified according to trough levels. Here 
again, the optimal trough levels are not yet known. Trough levels of $4-8 \mathrm{ng} / \mathrm{mL}$ [466], $5-10 \mathrm{ng} / \mathrm{mL}$ [375] or $10-15 \mathrm{ng} / \mathrm{mL}$ [374] have been recommended. Evidence-based recommendations concerning the frequency of trough level determination are not available. In the initial treatment phase, more frequent trough level measurement will be required, e. g., weekly. If trough levels and dosage are stable, the frequency of trough level determination can be reduced. The correlation between trough levels and side-effect rates is often a limiting factor and not all patients will tolerate a high trough level $(10-15 \mathrm{ng} / \mathrm{mL})$ in the long term.

\section{Infectious problems}

The understanding of the pathogenesis of ulcerative colitis has been significantly broadened over the last few decades. Clinical and experimental findings suggest that a gastrointestinal barrier malfunction and the dysregulation of the immune system play a causal role. There is, however, no evidence that patients with ulcerative colitis themselves develop an immune defect increasing their susceptibility to bacterial or viral infections. Rather, the therapy with immunosuppressive drugs causes partial deficits in the innate and acquired immune system, which can often lead to typical and opportunistic infections. In patients with a severe course of disease, the resulting malnutrition can intensify drug-induced immune deficiency.

\section{STATEMENT 4.1}

If several immunosuppressive drugs are given in combination, the risk of infections increases markedly with the addition of each additional drug. Elderly patients, patients with comorbidities, patients with a history of severe infectious disease and malnourished patients are particularly at risk from infections.

Evidence grade 3, strong consensus

\section{Background}

Each immunomodulatory drug bears an increased risk of infection for patients with ulcerative colitis. The risk is, however, not individually quantifiable [306]. A case control study analysing a total of 300 patients with IBD showed that the risk of opportunistic infection under monotherapy with steroids, azathioprine/6mercaptopurine or infliximab was three times higher compared to patients not receiving immunosuppressive therapy. However, combination therapy with two or three of the above drugs was associated with a drastic increase in the risk of infection (OR 14.5) [11]. Anti-integrin antibody therapy probably does not cause an increased risk of infection [467].

In estimating the risk of therapy-induced infections, it is often overlooked that systemic steroids carry a considerable risk of infection [468]. Rheumatological data show that the risk of infection is increased in patients taking a dose of more than $10 \mathrm{mg}$ prednisone or a cumulative dose of more than $700 \mathrm{mg}$, and in those with a therapy duration longer than two weeks [469, 470]. An analysis of about 500 patients with new-onset tuberculosis in- dicates that the risk of becoming infected with tuberculosis is increased 5-fold in patients under systemic steroid therapy compared to patients not receiving steroids. Moreover, the risk increase was dose-dependent, patients with a maximum daily steroid dose of less than $15 \mathrm{mg} /$ day having an approximately 3 -fold increase, whereas in those receiving more than $15 \mathrm{mg} /$ day, the risk was increased almost 8-fold [471].

IBD patients over 50 years of age are reported to have a three times greater risk of infection than patients under 25 years [ 11 , $472,473]$. Possible reasons for the heightened risk of infection in older individuals are changes in both the innate and adaptive immune systems [474]. Data from patients with rheumatoid arthritis show that comorbidities (e. g. kidney malfunction, diabetes mellitus) and prior severe infectious disease in the medical history represent additional risk factors for the occurrence of infections [475]. Diabetes has been described as a manifest risk factor for infection in patients with IBD [476]. Particularly in elderly patients, therefore, the risk of drug-induced infections must be carefully weighed up against the possibly lower surgery-associated risks of proctocolectomy.

\section{RECOMMENDATION 4.2}

At the time of first diagnosis or, at the latest, before starting immunosuppressive therapy, all patients should be screened for hepatitis B, tuberculosis and EBV infection. Tuberculosis screening is to be repeated prior to the introduction of biologic therapy.

Evidence grade 3, recommendation grade B, consensus

\section{RECOMMENDATION 4.3}

Before initiating immunosuppressive or immunomodulatory therapy for ulcerative colitis, a targeted past medical history, an X-ray examination of the lungs and an interferon gamma release assay (IGRA) should be performed to rule out active or latent tuberculosis infection.

Evidence grade 3, recommendation grade $B$, strong consensus

\section{Background}

Immunosuppressive therapy, including therapy with TNF antibodies, or chemotherapy can significantly increase the risk of hepatitis B (JBV) reactivation in HBV carriers. In HBsAg carriers, the incidence of $\mathrm{HBV}$ reactivation during or after chemotherapy is $15-50 \%$ [477, 478]. Although HBV reactivation occurs considerably less frequently in $\mathrm{HBsAg-negative} \mathrm{patients,} \mathrm{its} \mathrm{reactivation}$ has been described in $14-20 \%$ of anti-HBc- and anti-HBs-positive patients with lymphoma under chemotherapy [479]. A Spanish study reported chronic HBV infection in 3 of 80 patients with Crohn's disease. Two of the patients developed severe hepatitis after discontinuation of infliximab therapy, while the third died due to complications related to liver cirrhosis [480]. These examples show that the problem of reactivation not only has implications for long-term consequences (e. g. cirrhosis or hepatocellular 
carcinoma) but that the risk of acute exacerbation of hepatitis $B$ with a fulminant course is also increased [481]. Therefore, screening for HBsAg and anti-HBc antibodies is recommended in all patients prior to commencement of immunosuppressive therapy. In seronegative patients, vaccination is recommended. Preventive drug therapy with nucleoside/nucleotide analogues counteracts $\mathrm{HBV}$ reactivation and is therefore indicated in $\mathrm{HBsAg}$-positive patients. In HbsAg-negative and anti-HBc-antibody-positive patients, close monitoring of ALT and HBV DNA is recommended. For details on preventive drug therapies and the management of HbsAg-negative and anti-HBc-antibody-positive patients, current guidelines should be consulted [482].

Before initiating immunosuppressive therapy - which includes, strictly speaking, high-dose steroid therapy - the patient should be screened for tuberculosis. If possible, screening should be performed as soon as ulcerative colitis is diagnosed. In principle, either the Tuberculin Skin Test (TST) or Interferon-Gamma Release Assay (IGRA) can be used for immunodiagnostic testing, possibly in combination with a chest $X$-ray examination. All of the available testing methods have weaknesses. Although the risk of false-negative results using IGRA is low, this cannot be excluded, especially in patients with severe lymphopoenia and immunosuppression [483]. TST can produce false-negative results in patients with reduced immunocompetence; vaccinated patients have false-positive results [484]. Alongside the IGRA, X-ray examinations of the lungs may reveal signs of past, untreated tuberculosis with no evidence of activity (calcified nodules, peak fibrosis, pleural weals), which are considered an indication for chemoprevention (see recommendation 4.19).

The risk of tuberculosis is probably greater under therapy with TNF antibodies compared to other immunomodulatory/-suppressive drugs. Accordingly, it is recommended that tuberculosis diagnostics be repeated prior to the initiation of TNF antibody therapy, to rule out the possibility that tuberculosis infection may have occurred since initial IBD diagnosis and tuberculosis screening. A number of professional societies recommend annual tuberculosis screening in patients under continual anti-TNF therapy, given the conversion rate of tuberculosis-screening-negative to tuberculosis-screening-positive patients of up to $30 \%$ [485]. Due to the low prevalence of tuberculosis in Germany, this recommendation cannot be followed. However, individuals with an increased risk of exposure (e.g. airport personnel, employees of pulmonological clinics, long-distance travellers to tuberculosis-endemic regions, etc.) should undergo screening on an annual basis.

Even though epidemiological evidence of an increased risk of tuberculosis under therapy with anti-integrin or JAK-inhibitor therapy is lacking (in all of the pivotal trials, patients with latent tuberculosis were excluded), tuberculosis should be ruled out prior to therapy initiation, not least on account of the drug approval stipulations.

Infection with the Epstein-Barr virus (EBV) usually occurs during childhood and often takes an asymptomatic course. In adolescents and adults, the primary infection manifests as infectious mononucleosis in $30-60 \%$ of cases. At the age of forty, approximately $95 \%$ of the population are infected with EBV. Following a symptomatic or asymptomatic infection, EBV persists lifelong in B-cells in the circulation under the control of EBV-specific cytotoxic cells [486]. If the immunosurveillance of the T-cells is disrupted, e. g. in a post-trans- plantation setting, the proliferation of EBV-infected B-cells is enhanced, resulting in an increased risk of developing post-transplantation B-cell lymphoma or lymphoproliferative disease (PTLD) [487]. Primary EBV infection in the first few years after transplantation is associated with a considerably increased risk of PTLD [488, 489].

\section{RECOMMENDATION 4.4}

Upon initial diagnosis, or, at the latest, before immunosuppressive therapy is initiated, vaccination status should be reviewed and updated as appropriate. Non-live vaccines are considered safe under immunosuppressive therapy, whereas live vaccines are contraindicated. In view of the above, vaccinations against pneumococcal infection, hepatitis B, influenza and pandemic flu should be administered analogous to the recommendations of the Robert Koch Institute (RKI) ("Vaccinations under immunosuppression").

Evidence grade 2, recommendation grade B, strong consensus

\section{Background}

Patients with ulcerative colitis have an increased risk of contracting vaccine-preventable infectious disease and also of being hospitalised as a consequence [490]. Several healthcare research projects found that many patients with IBD have only inadequate vaccination coverage. The main caveat against vaccination is an often unfounded fear of side effects. Some patients are also afraid that vaccinations may cause exacerbation of the underlying disease; however, in principle, the majority of patients are prepared to undergo vaccination as recommended [491].

In all patients with ulcerative colitis, compliance with the general vaccination recommendations of the RKI should be reviewed at diagnosis and subsequently at regular (e. g., annual) intervals. At the time of diagnosis, it is frequently still possible to catch up on live vaccinations (especially measles, rubella, varicella) which have previously been missed. After initiation of immunosuppressive therapy, live vaccinations are formally contraindicated, although probably nevertheless safely practicable [492]. It should be kept in mind that patients under ongoing immunosuppression, particularly under anti-TNF or combined immunosuppressive therapy, have reduced response rates to vaccination and therefore require appropriate monitoring [493]. Depending on the severity of disease and the therapy, patients should be annually vaccinated against influenza A/B. Inoculation against pneumococcal infection is indicated in all patients who require, or may potentially require, immunosuppressive therapy. The initial vaccination should be performed with PCV13 and the booster with PPV23. Vaccinations against meningococci and Haemophilus influenzae may be appropriate in individual cases, as well as specific travel vaccinations.

\section{RECOMMENDATION 4.5}

Patients treated with triple immunosuppressive therapy should receive a PJP (Pneumocystis jiroveci pneumonia) prophylaxis. Evidence grade 4, recommendation grade $B$, consensus 


\section{Background}

The onset of PJP has been observed during therapy with steroids, thioguanines, MTX, calcineurin inhibitors or biologics, with calcineurin inhibitor therapy and combination therapies appearing to pose the highest risk [494]. The available retrospective studies indicate that the absolute incidence is relatively low: in the Olmsted County cohort, only 3 cases of PJP were observed in 937 patients (6066 patient years' follow-up), despite irregular prophylactic therapy [495]. In an analysis of data from health insurance companies, a risk increase from 3 to 10.6/100 000 person years was found for IBD patients in comparison to the general population, and a further increase up to 32/100000 person years in IBD patients under immunosuppressive therapy [496]. Systematic data describing the risk of PJP under specific immunosuppressive regimes are lacking. Therefore, the recommendation is also based on experience from patients with other underlying diseases (see also: [330]).

$\mathrm{TMP} / \mathrm{SMX}$ prophylaxis is highly effective for PJP prevention in children and adults with haematological disease, bone marrow transplantation or organ transplantation [497]. In the 2014 ECCO guidelines “Opportunistic Infections”, PJP prophylaxis is recommended under triple immunosuppression incorporating a calcineurin inhibitor or TNF antibody, whereas under double immunosuppressive therapy with a calcineurin inhibitor, a prophylaxis should be considered [330]. While a CD4 cell count $<200 / \mu \mathrm{L}$ represents an important risk factor for PJP in numerous HIV-independent diseases [498], there is insufficient evidence to support a firm recommendation for CD4 monitoring in IBD patients. The recommended prophylactic regimen, sulphamethoxazole/trimethoprim $(800 / 160 \mathrm{mg}) 3$ times weekly, has been shown to be well tolerated [497, 499]. In cases of sulphonamide allergy, it is advisable to consult a specialist in infectiology.

\section{RECOMMENDATION 4.6}

In patients with known ulcerative colitis with a severe acute episode, atypical symptoms, a refractory disease course, or prior to the intensification of immunosuppressive therapy, microbiological diagnostics including examination for Clostridium difficile should be carried out.

Evidence grade 3, recommendation grade B, strong consensus

\section{Background}

Routine diagnostics for $\mathrm{C}$. difficile are not recommended in patients with mild disease flares, since $C$. difficile infection (CDI) is uncommon in this situation [42, 500]. However, hospitalised patients and patients with prior antibiotic therapy have a higher rate of CDI. IBD patients with CDI have longer hospital stays and a four-fold increased mortality rate in comparison to IBD patients without CDI. Screening for $\mathrm{C}$. difficile is therefore to be recommended [52, 337, 501].

Glucocorticoids (OR 2.5), immunomodulators (OR 1.6) and TNF antibodies (OR 2.7) are risk factors for severe CDI [502 504]. Empirical data from IBD patients and from the field of transplantation medicine show that immunosuppressive therapy is associated with a higher incidence (OR 2.5 for AZA/6-MP or MTX) and increased severity of the infection $[52,505]$. Steroid therapy increased the risk of CDI threefold in comparison to AZA/6-MP and MTX in 10662 patients with IBD (RR 3.4; $95 \% \mathrm{Cl} 1.9-6.1$ ) [503]. Additional risk factors include (prior) antibiotic therapy or nasogastric tube feeding/PEG [506]. IBD patients with CDI and concomitant proton pump inhibitor therapy have an almost four times higher risk of recurrence of $C$. difficile colitis [339].

\section{RECOMMENDATION 4.7}

Diagnostic procedures to detect infection with Clostridium difficile should be carried out promptly using a sensitive testing method.

Evidence grade 3, recommendation grade $B$, strong consensus

\section{Background}

C. difficile diagnostics should be performed promptly using a sensitive detection method [338]. As a rule, one soft-to-liquid stool sample is sufficient; formed stool diagnostics are not indicated. In special situations, e. g., in patients with ileus, a rectal swab can be used. There is no generally accepted algorithm for biochemical diagnostics, as confirmed by the current guidelines of the European Society of Clinical Microbiology and Infectious Diseases (ESCMID) [507]. In principle, test procedures can be differentiated into two distinct types, one-step or multistep. Time-saving diagnostic methods such as direct antigen detection in stool (glutamate dehydrogenase $[G D H]$ ), the direct detection of toxins $A / B$ (so-called rapid tests) or biomolecular test methods are suitable for toxin detection [338]. Detection by GDH is unspecific and must be confirmed by a second, specific test. In patients with severe, notifiable infection, patients with recurring infection, and in the context of nosocomial outbreaks, additional cultural verification should be sought. This enables the detection of epidemiologically particularly important strains (e. g., ribotype 027) and antibiotic resistance testing [508]. It is also important to note that a stool test negative for $C$. difficile toxins is not sufficient to rule out pseudomembranous colitis.

In patients with ulcerative colitis and other patients under immunosuppression, smaller quantities of toxins (which are undetectable in the diagnostic tests) are apparently sufficient to trigger pseudomembranous colitis. Repeated stool studies for toxin detection or the performance of $\mathrm{C}$. difficile culture followed by toxin detection are therefore recommended [509]. Sigmoidoscopy can also be useful, especially in patients with refractory disease and negative stool cultures [508]. However, negative findings do not rule out infection, especially since the typical endoscopic findings with formation of pseudomembranes are often lacking.

\section{RECOMMENDATION 4.8}

Depending on its clinical severity, recommendations for specific primary therapy of CDI are as follows:

- Patients with mild to moderate disease and without risk factors for a severe disease course can be treated with metronidazole $3 \times 400 \mathrm{mg} /$ day p. $\mathrm{o}$. for a minimum of 10 days. 
- Patients with severe disease or with predictors of a severe disease course should be treated with vancomycin $4 \times 125-250 \mathrm{mg} /$ day p. 0 . for a minimum of 10 days.

- In patients with recurrent disease or additional risk factors for complications (immunosuppression, comorbidity, necessity for additional antibiotic therapy), treatment with fidaxomicin $2 \times 200 \mathrm{mg} /$ day p. o. can be considered.

- In patients with recurrent or therapy-refractive Clostridium difficile infection, faecal microbiota transplantation can be performed.

Expert consensus, recommendation open, strong consensus

\section{Background}

When treating CDI, it is important to maintain contact isolation and coat and glove care, and to ensure that hands are cleaned with soap and sporicidal disinfectants [510]. While there are a variety of recommendations concerning options for drug therapy of patients with CDI, almost all include antibiotic therapy with metronidazole or vancomycin, varying only in the drug dose, duration of therapy, and type of antibiotic to be given as the primary therapy. Controlled studies investigating drug therapies of $C$. difficile in patients with IBD are lacking. The recommendations given above are in line with the German S2k guideline, "Gastrointestinal Infections and Whipple's disease" [338].

Depending on the severity of disease, primary therapy with metronidazole or vancomycin is recommended. If the clinical course permits, any ongoing antibiotic treatments should be discontinued before beginning CDI therapy. In patients with symptoms of mild to moderate disease, a ten-day therapy with $400-500 \mathrm{mg}$ metronidazole p. o. q 8 hours is recommended. If no improvement is seen after $3-5$ days, therapy must be presumed to have failed, in which case a switch to e. g. vancomycin p. o. should be considered. In patients with severe CDI (fever, chills, haemodynamic instability, peritonitis, ileus, leucocytosis $>15 \times 10^{9} / \mathrm{L}$, left shift, increased lactate, toxic megacolon), an initial antibiotic therapy with $125 \mathrm{mg}$ vancomycin q 6 hours p. o. for 10 days is recommended. An increased dose of vancomycin offers no additional benefit [511]. In patients who cannot be treated with oral antibiotics, vancomycin can be administered into the colon (500 mg in $100 \mathrm{~mL}$ saline solution q $4-12$ hours) and/or via a nasal tube (500 mg q 6 hours).

Ongoing immunosuppressive therapy in patients with CDI should be paused or stopped if possible, since IBD patients under immunosuppression have been shown to have an increased morbidity (e. g., colectomy, toxic megacolon, bowel perforation) and mortality in comparison to a control group without immunosuppression [339]. However, it remains unclear whether immunosuppressive therapy per se denotes a severe disease course with a greater potential for complications.

In patients with $C$. difficile infection whose IBD is not in remission, persisting diarrhoea can hinder clinical assessment of the response to antibiotic therapy. Renewed microbiological stool diagnosis cannot be used to measure the success of antibiotic therapy, since both $C$. difficile and toxins $A$ and $B$ can be detected in stool even weeks after successful treatment [512]. However, if a recur- rence of the infection is suspected, stool diagnostics should be repeated. Resistance to metronidazole has been reported and, although rare, must be considered in the case of therapy failure [513].

Therapeutic success rates of metronidazole and vancomycin were compared in a retrospective observational study of 62 patients with ulcerative colitis. Patients with mild infections had fewer readmissions to hospital ( $0 \%$ versus $31 \%$ ) and shorter hospital stays ( 6 versus 14 days) when treated with vancomycin $(n=13)$ compared to metronidazole therapy $(n=29)$. Patients with severe infection also had fewer readmissions to hospital ( $0 \%$ versus $70 \%$ ), but tended to have longer hospital stays (19 versus 11 days) under vancomycin therapy $(n=9)$ compared to metronidazole therapy $(n=10)$. This study provided the first evidence to support the administration of vancomycin as primary therapy for $\mathrm{CDI}$ in patients with ulcerative colitis [514].

Compared to vancomycin, in patients without IBD, fidaxomicin has a comparable overall primary response rate, but also a lower recurrence rate $[515,516]$. Its major disadvantage is its high cost. Furthermore, fidaxomicin has not yet been approved for therapy of $C D I$ in patients with IBD. A pharmacokinetic study published as a short communication showed no differences in the resorption of fidaxomicin in CDI patients with and without IBD [517]. At the present time, other drug therapies for CDI, such as rifaximin, fusidic acid or nitazoxanide, cannot be recommended [518]. Whether the additional administration of probiotics during antibiotic therapy is protective against $C D I$ in patients with ulcerative colitis, as has been described for patients without IBD, is unclear [519].

Fulminant CDI has a serious prognosis. Therefore, in patients with severe clinical symptoms, the possibility of surgical intervention should be considered early in the context of interdisciplinary care.

Treatment for relapse of CDI is the same as that used in the primary episode. A further relapse should be treated directly with vancomycin $125 \mathrm{mg} \mathrm{q} 6$ hours for 10 days [338]. The procedure in patients with more than two episodes remains unclear. The risk of recurrence in these patients is especially high. $40-60 \%$ of patients who relapse a second time go on to experience further $\mathrm{CDI}$ episodes. Based on case series, patients with multiple CDI recurrences have been successfully treated with vancomycin in various tapering and/ or pulse regimens [520]. Theoretical considerations suggest that by administering vancomycin on alternate days, the clostridial spores remaining in the intestine can be dispersed on the "vancomycinfree days" and be killed off subsequently in their vegetative form.

Successful therapeutic transfer of stool from a healthy donor to a patient with CDI was first described as early as 1958 [521]. Numerous studies have confirmed the effectiveness of stool transplantation, now known as faecal microbiota transplantation (FMT). The first randomised, controlled study showed FMT to be significantly superior to a conventional relapse therapy with vancomycin in patients with multiple CDI relapse [522]. In this indication, FMT is explicitly recommended in current European guidelines as a therapeutic alternative. A retrospective multicentre study including 31 patients with UC and 35 patients with Crohn's disease demonstrated FMT to be efficacious in the treatment of CDI, with success rates of $79 \%$ after the first FMT, $88 \%$ after the second FMT und $90 \%$ after the third FMT [523]. For recurring CDI, FMT is probably less effective in IBD patients than in patients without IBD. Moreover, approximately $15-20 \%$ of IBD patients experi- 
ence a disease flare after FMT [524]. Extraintestinal manifestations of IBD can also occur for the first time in this context [525].

There are still some remaining safety concerns, which it has not been possible to entirely eliminate, despite (cost-intensive) donor screening tests (e. g., for HIV, hepatitis, enteropathogens, stool parasites, multiresistant pathogens). A national, internet-based registry has been established in Germany to collect additional information on the clinical efficacy and long-term safety of FMT, including patient characteristics, procedural details of FMT and long-term patient follow-up data (for details, see: www.kim4.uniklinikum-jena.de).

\section{RECOMMENDATION 4.9}

If possible, EBV-seronegative adult patients should not be treated with thiopurines.

Expert consensus, recommendation, consensus

In children, the therapeutic decision represents a special situation and requires careful risk assessment.

Expert consensus, open recommendation, majority approval

\section{Background}

It is generally accepted that the risk of developing lymphoma is increased in patients with IBD, especially those treated with thiopurines. Findings of the CESAME cohort study, with a population of 20000 patients, indicate the risk of lymphoma to be increased five-fold under thiopurine therapy [526]. However, the absolute risk was low, with one additional lymphoma occurring every 300 1400 patient years. Nevertheless, the importance of EBV infection is not to be underestimated. In the CESAME cohort of patients treated with thiopurines, 12 of 15 lymphomas were PTLD-like and, as a rule, associated with EBV. In this situation, primary EBV infection is especially challenging: Of 6 patients under 50 years of age, 2 males developed fatal infectious mononucleosis with lymphoproliferative sequelae [526]. Similar serious complications have previously been reported in casuistic studies [527 - 529].

\section{RECOMMENDATION 4.10}

In patients with EBV (re-)infection, immunosuppressive/immunomodulatory therapy should be suspended.

Expert consensus, recommendation, consensus

\section{RECOMMENDATION 4.11}

In patients with EBV-associated lymphoproliferative disease, immunomodulatory therapy with thiopurines should be discontinued.

Expert consensus, recommendation, strong consensus

\section{Background}

In patients who develop a primary EBV infection under immunosuppression, it is recommended to interrupt immunosuppressive therapy and, if appropriate, to administer antiviral therapy, in consultation with a specialist for infectious diseases. If atypical infiltrates are detected in the intestinal mucosa of patients who are EBV-positive, a reduction in immunosuppression may contribute to the control of virus replication and the disappearance of infiltrates [530]. Haemophagocytic lymphohistiocytosis (HLH), also known as macrophage activation syndrome (MAS), has been reported as a severe and potentially lethal complication of acute EBV infection under thiopurines. A contemporary review summarises 50 cases with a mortality rate of $30 \%$ [531]. In addition to the interruption of immunosuppressive therapy, patients with HLH may require treatment with anti-lymphoproliferative drugs [532 - 534].

\section{RECOMMENDATION 4.12}

In patients who inadequately respond to drug therapy, especially systemic steroid therapy, diagnostic tests for new onset or reactivation of CMV infection should be carried out.

Expert consensus, recommendation, strong consensus

\section{Background}

The seroprevalence of CMV in the general population is 70 $100 \%$, rising with age. Since this figure is the same for patients with ulcerative colitis, it is not useful to perform screening independent of the clinical situation. Several studies have shown increased evidence of CMV in patients under systemic steroid therapy [326, 535, 536]. In many cases, it is difficult to assess whether steroids facilitate CMV replication or whether pre-existing increased CMV replication leads to the supposed clinical need for steroid therapy and possible refractoriness. For other immunosuppressants, such as thiopurines, IL2 inhibitors or TNF antibodies, the evidence is even more inconsistent and/or sparse. In patients treated with these drugs who show inadequate clinical improvement of ulcerative colitis activity and/or signs of systemic CMV infection (particularly fever and leucopoenia), CMV diagnostics should likewise be performed.

Due to the currently inconsistent evidence, CMV diagnostics should only be carried out if antiviral therapy is considered useful upon detection of CMV. This decision depends largely on the severity of clinical symptoms [331, 537].

\section{RECOMMENDATION 4.13}

Diagnostic tests should include immunohistochemical CMV detection from endoscopically obtained samples and/or biomolecular detection from tissue samples, or biomolecular detection from whole blood.

Evidence grade 3, recommendation grade $B$, consensus

\section{Background}

CMV diagnosis can be based on whole blood, serum or bioptic sample detection methods. In recent years, direct detection in the inflamed mucosa has become increasingly important (tissue CMV-PCR, immunohistochemical CMV testing), while serological 
methods (serum CMV-IgM) or detection in whole blood (CMVPCR, pp65) have been less thoroughly researched. Samples for immunohistochemical or biomolecular analysis should, if possible, be taken from ulcerated tissue - ideally from the base or edge of the ulcer [538-541].

\section{RECOMMENDATION 4.14}

CMV detection alone should not be considered an indication for therapy. The indication for therapy can be justified by the clinical context. Acute therapy should be administered for a minimum of 14 days.

Evidence grade 2, recommendation grade $B$, consensus

\section{Background}

None of the diagnostic procedures has a defined cut-off point after which sufficient diagnostic precision and the necessity for antiviral therapy can be presumed. In addition, tissue CMV detection may not influence the course of ulcerative colitis in patients with mild subclinical CMV reactivation or continual CMV replication. Consequently, evidence of CMV in patients with active ulcerative colitis justifies the necessity for antiviral therapy only in the context of the overall clinical picture and in consideration of additional individual factors (e. g. clinical severity of UC activity, duration and intensity of steroid therapy) [56, 57, 542, 543]. Since, as yet, there is no accepted cut-off limit for virus load in the tissue PCR for CMV, the possibility of false-positive results must be considered, especially when titres are low.

There is no approved drug therapy for CMV colitis or CMV reactivation in patients with ulcerative colitis. Common off-label applications are $5 \mathrm{mg} / \mathrm{kg}$ ganciclovir i. v. q 12 hours, or a primary (or secondary, after successful primary response to ganciclovir) oral therapy with $900 \mathrm{mg}$ valganciclovir q 12 hours for 2 (to 3) weeks. In case of insufficient response, a 2 - 3 week therapy with foscarnet (e. g. $60 \mathrm{mg}$ q 8 hours i. v. over at least one hour) can be considered, whereby its inferior side-effect profile must be kept in mind [544]. The extent to which a "prophylactic" daily dose of e. g. $450 \mathrm{mg}$ valganciclovir adjuvant to immunosuppressive therapy may be useful, and for how long, have not yet been investigated.

\section{RECOMMENDATION 4.15}

In patients with severe manifestations of CMV disease, in particular severe CMV colitis, meningoencephalitis, pneumonitis or hepatitis, concurrent immunosuppression should be interrupted at least until the virus is no longer detectable. If symptoms of ulcerative colitis relapse with CMV detection are limited to the intestine, immunosuppressive therapy can be continued or modified.

Evidence grade 2, recommendation grade B, strong consensus

\section{Background}

Even after detailed clinical differentiation, it is often impossible to define CMV as the sole cause of an acute and possibly steroid re- fractory flare of ulcerative colitis on the basis of positive CMV detection in the inflamed tissue. Therefore, an individualised multimodal therapy with e. g. thiopurines or TNF antibodies should be administered concomitant or subsequent to antiviral therapy [542]. Current data indicate the risk of exacerbating a CMV infection or requiring colectomy under concurrent immunosuppression to be relatively low [545]. If results of plasma CMV PCR under antiviral therapy turn negative in the context of inadequate clinical improvement of ulcerative colitis, it may possibly be taken as a signal for the intensification of immunosuppressive therapy [544]. Intensification of therapy with a TNF antibody is - in comparison to azathioprine associated with a markedly lower increase in the viral load of CMV. In the light of the increased risk of later colectomy in UC patients with clinically relevant CMV reactivation, it may be appropriate to consider proctocolectomy, even at this early stage [546].

Patients with severe intestinal and extraintestinal manifestations of CMV infection - both of which are frequently accompanied by fever - should be admitted to a clinic with interdisciplinary expertise. In line with the guidelines of the "Transplantation Society International CMV Consensus Group”, we would suggest reducing or interrupting immunosuppressive therapy in this situation [547].

\section{RECOMMENDATION 4.16}

Upon reintroduction, continuation or intensification of immunosuppressive therapy, patients with confirmed CMV disease should receive a prophylactic therapy to prevent disease recurrence. This therapy should be administered for 4-8 weeks subsequent to completion of the acute therapy.

Expert consensus, recommendation grade $B$, strong consensus

\section{Background}

There is no prospective randomised study evaluating the usefulness of antiviral prophylaxis for relapse prevention following successful elimination of the virus of clinically relevant CMV infection in patients with UC and subsequent reintroduction or modification of immunosuppressive therapy. Based on recommendations from transplantation medicine, a daily dose of 450 $900 \mathrm{mg}$ valganciclovir may be administered as a prophylaxis against disease recurrence [547].

\section{RECOMMENDATION 4.17}

Immunosuppressive therapy should not be initiated in patients with an active VZV infection.

Expert consensus, recommendation, strong consensus

\section{RECOMMENDATION 4.18}

If active VZV infection arises during immunosuppressive therapy, it should be treated immediately. If possible, immunosuppressive therapy should be interrupted.

Expert consensus, recommendation, strong consensus 


\section{Background}

At initial diagnosis, all patients should be questioned with regard to their past medical history for VZV, and their vaccination pass should be checked for immunisation against varicella. Under immunosuppression, acute chicken pox infection has a pronounced risk of complications (e. g., varicella pneumonia) with increased mortality. Thus, patients without a history of chicken pox and without VZV titre should, if possible, be vaccinated before beginning immunosuppressive therapy. Much more common in adult patients is the reactivated form of VZV, which carries a risk of long-term neurological complications (neuralgia) [548].

The duration and application route (oral or i. v.) of therapy with antiviral substances (e. g., aciclovir $5 \times 800 \mathrm{mg}$, valaciclovir $3 \times 1000 \mathrm{mg}$, famciclovir $3 \times 250 \mathrm{mg}$, brivudine $1 \times 125 \mathrm{mg}$ ) depends on the severity of disease. As a rule, a therapy duration of 5 - 10 days, or until skin lesions have formed a crust, is sufficient [549]. Immunosuppression can then be recommenced if no new efflorescence arises, in other words, if all lesions have crusted over. There are, as yet, no data to support the continuation of antiviral therapy in a "prophylactic" dose.

Passive immunisation with VZV immunoglobulin should be considered within 3 days of exposition in immunosuppressed patients (e. g. a mother under thiopurine/TNF antibody therapy) with a high risk of infection and complications (combined immunosuppression, close contact with infected individuals, advanced age).

\section{RECOMMENDATION 4.19}

If a latent tuberculosis infection (LTBI) is detected by interferon gamma release assay (IGRA), chemopreventive therapy with isoniazid should be carried out according to the recommendations of the RKI.

Expert consensus, strong recommendation, strong consensus Immunosuppressive therapy should not be initiated until at least 4 weeks after chemopreventive therapy is started.

Expert consensus, recommendation, strong consensus

\section{Background}

Recommended is a therapy with $5 \mathrm{mg} / \mathrm{kg}$ (maximum $300 \mathrm{mg}$ ) isoniazid (INH) daily for 9 months. In rare instances, patients receiving this therapy can develop INH hepatitis $(0.15 \%)$. Patients intolerant to isoniazid can alternatively be administered a chemopreventive therapy with rifampicin over a duration of 4 months, whereby this regimen has been inadequately evaluated in patients under TNF inhibition. If the patient has immigrated from a country where INH resistance is known to be high (e.g., Russia), chemopreventive therapy with rifampicin and pyrazinamide over 3 months is recommended.

\section{RECOMMENDATION 4.20}

If active tuberculosis is confirmed, combination therapy is to be initiated in accordance with RKI guidelines.

Expert consensus, strong recommendation
Immunosuppressive therapy, especially anti-TNF therapy, should ideally only be initiated after the completion of tuberculosis therapy, and if so, always on the basis of restrictive indication criteria.

Expert consensus, recommendation, strong consensus

\section{Background}

Concerning the treatment of active tuberculosis, we recommend referring to guidelines issued by the RKI German Central Committee for the Control of Tuberculosis, or the current American guideline [550, 551]. Whether it is possible to initiate TNF antibody therapy during ongoing tuberculostatic therapy is not known. Anti-TNF therapy should ideally not be introduced until after the completion of tuberculosis therapy. Especially in this situation, a critical discussion with the patient and the application of restrictive indication criteria are advised.

\section{RECOMMENDATION 4.21}

New-born babies of mothers treated with TNF antibodies during pregnancy should not be immunised with live vaccines (rotavirus) for at least 9 months.

Expert consensus, strong recommendation, strong consensus

\section{Background}

TNF antibodies (with the exception of Certolizumab) cross through the placenta and thus reach therapeutic levels in the foetus. Trough levels in new-borns at birth depend upon the timepoint of the last application during pregnancy [552]. The majority of immunisations recommended by the STIKO (Constant Committee on Vaccination, Robert Koch Institute) during the first few months of life are based on non-live vaccines. These are considered to be safe. For the past few years, however, the recommendations have included a live oral immunisation against rotavirus during the first few weeks. This must be avoided. Mothers need to be made aware of this during pregnancy. Live vaccinations for measles, mumps, rubella and varicella are not recommended until the age of 11 - 14 months, and are thus generally unproblematic, since by this time there is total clearance of the drug in the baby.

\section{Surgery}

\subsection{Surgical Techniques}

\section{RECOMMENDATION 5.1.1}

Restorative proctocolectomy should be performed as the standard surgical procedure.

Evidence grade 3, recommendation grade B, strong consensus 


\section{Background}

Over the past 35 years, restorative proctocolectomy has become established as the standard surgical procedure in patients with therapy-refractory ulcerative colitis or development of ulcerative colitis-associated colorectal cancer. Ileoanal pouch surgery provides the best possible quality of life for the patient, with an average of 5-6 stools per day, and maintains continence in over $90 \%$ of patients $[553,554]$.

\section{RECOMMENDATION 5.1.2}

As a rule, restorative proctocolectomy should be performed using a protective ileostoma. A single-step procedure should only be carried out in selected cases.

Evidence grade 2, recommendation grade B, strong consensus

\section{Background}

Anastomotic insufficiency occurs in $10 \%$ of patients who have undergone ileopouch-anal anastomosis. Both the absolute prevalence rate and the clinical implications of such leaks can potentially be reduced by a protective stoma. While leaks are presumed to compromise the eventual pouch function, the evidence is conflicting. RCTs with a sufficiently large population are not available. A large contemporary registry study from Denmark with a 33-year time horizon demonstrated a significant association between surgery without protective stoma and subsequent pouch failure [555].

In order to identify selection criteria to define cases where a protective stoma may be unnecessary (single-step procedure), two "high-volume" centres jointly analysed their pouch databanks. Just under $15 \%$ of the 3733 patients had received no protective stoma. Forgoing stoma construction was significantly associated with stapler anastomosis, lack of steroids in the preoperative medication, familial adenomatous polyposis (FAP) or carcinoma as the indication for surgery, female gender and age below 26 years. Postoperative morbidity, including the rate of anastomotic insufficiency, did not differ between patients with and without stoma [556].

In conclusion, in patients undergoing restorative proctocolectomy due to therapy refractory disease, a stoma should generally be used, since the potential disadvantages of a stoma are more than compensated for by their advantages in these patients who are, as a rule, seriously ill [557].

\section{RECOMMENDATION 5.1.3}

The J-pouch should be the pouch construction of choice, since it is the simplest to create and offers a function similar to other constructs in the long-term.

Evidence grade 1 , recommendation grade $B$, strong consensus

\section{Background}

The available studies show no clear functional advantage for the J-design. In a meta-analysis of 18 non-randomised studies (NRS) with a total of 1519 patients, there was no difference in the rate of early post-operative complications. With respect to stool frequency, the $\mathrm{W}$ - and the S-pouch were superior to the J-pouch, whereas the $S$-pouch was found to have disadvantages in terms of pouch emptying disturbances [558]. In a more recent randomised study (W-versus J-pouch), the median 24-hour stool frequency after one year was significantly higher for the J-pouch than the W-pouch (7 vs. 5). However, after 9 years, there was no significant difference. All other parameters which were evaluated, including incontinence, use of incontinence pads and quality of life, were similar [559]. The K-pouch was compared with the $\mathrm{J}$-pouch in a small randomised study from Norway, whereby no significant functional differences were found [560].

Although the studies mentioned above suggest that other pouch configurations tend to have advantages, especially in the short term, the J-pouch remains the standard pouch due to its simple design. Furthermore, pouch emptying problems, which are clinically very agonising and occur in a considerable proportion of patients with S- or W-pouch, appear to have been largely neglected in the available studies.

\section{RECOMMENDATION 5.1.4}

Free or contained perforation should be considered an emergency indication for surgery.

Evidence grade 2, recommendation grade B, strong consensus

\section{Background}

Free or contained perforation is the most severe complication of ulcerative colitis. Evaluation of clinical symptoms is frequently hampered by concurrent immunosuppressive or antibiotic therapy. Therefore, operative therapy should be performed before the onset of septic complications. In half of affected patients, perforation is not preceded by a megacolon. Despite surgical therapy, perforation currently bears a mortality rate of up to $27 \%$ [561, 562]. The most crucial factor in reducing the mortality of this extremely severe complication is timely surgery [319, 392, 563]. In emergency surgery, colectomy with blind closure of the rectum and permanent ileostoma is the primary standard procedure (see also 5.1.5. and 5.1.6.) [564, 565].

\section{RECOMMENDATION 5.1.5}

Patients with a persistent need for transfusion due to therapy-refractory bleeding should undergo surgery as a matter of urgency. Evidence grade 2, recommendation grade B, strong consensus

\section{Background}

Severe bleeding occurs with an incidence of up to $4.5 \%$ in patients with UC [566] and accounts for up to $5 \%$ of emergency colectomies. Indications for surgery are massive initial bleeding with circulatory instability requiring treatment with catecholamine, or a persisting transfusion requirement of more than 4 erythrocyte concentrates per 24 hours. In children, the need for 45- $60 \mathrm{~mL}$ erythrocyte concentrate (EC)/ kg BW in 24 hours, or in case of continued bleeding, $30 \mathrm{~mL}$ EC/ kg BW over 2 - 3 days, must 
be considered life-threatening. If possible, children with a severe acute flare of ulcerative colitis should be referred to a paediatric unit with gastroenterological and surgical expertise. Should one of the aforementioned constellations arise, the patient should undergo urgent colectomy (within 24 hours), usually as a subtotal colectomy with resection in the proximal rectum [392].

\section{RECOMMENDATION 5.1.6}

Patients with a fulminant flare refractory to drug therapy should undergo urgent surgery.

Evidence grade 3, recommendation grade B, strong consensus

\section{Background}

Diagnosis of the refractory fulminant flare is interdisciplinary and based on the criteria of severe colitis according to Truelove and Witts. However, there is no uniform definition. Interpretation of the literature is therefore difficult. Radiologically, colon dilatation of $6 \mathrm{~cm}$ indicates a toxic megacolon [323, 567]. A therapy-refractive fulminant flare is, at minimum, a relative indication for surgery if no substantial improvement in disease intensity can be achieved, in spite of conservative intensive care and drug therapy with high-dose steroids over a duration of 72 hours. Surgical therapy is a useful alternative to the further intensification of drug therapy with calcineurin inhibitors or TNF antibodies. It should be taken into consideration that an additional intensification of drug therapy with calcineurin inhibitors or TNF antibodies can delay the need for surgery for at least 1 year only in up to $42-65 \%$ of cases [399, 568]. In a multivariate analysis, it has also been shown that later surgery ( 8 versus 5 days) leads to a significant increase in major complications in severe, acute colitis [319].

In conclusion, in patients with fulminant colitis, intensified drug therapy can be applied for a maximum of $5-7$ days, as long as the patient's condition does not deteriorate. Patients who deteriorate under therapy require urgent surgery within 24 hours, in order to minimise mortality and morbidity. The time frame for conservative therapy in patients with toxic megacolon is considerably more limited, and should not exceed 48 to 72 hours, at a maximum. Here again, patients who deteriorate or fail to improve under therapy should undergo surgery as a matter of urgency [562].

Children with a fulminant flare of ulcerative colitis should be referred to a specialist paediatric unit with gastroenterological and surgical expertise. Fulminant colitis is rare in children, and the clinical symptoms differ considerably from those commonly seen in adults. Calculation of the paediatric ulcerative colitis activity index (PUCAI) can be useful [569]. A PUCAI score of 45 points or more on day 3 , or 70 or more on day 5 , indicates "non-response to steroids" with high sensitivity and specificity [570].

\section{RECOMMENDATION 5.1.7}

Patients whose disease is refractory to immunosuppressants or biologics should undergo surgery.

Evidence grade 2, recommendation grade B, strong consensus

\section{Background}

Failure of intensified conservative therapy (immunosuppressants and/or biologics) is an indication for surgical removal of the colon. The operation can be a two- or three-step procedure, depending on the duration and intensity of premedication and the clinical symptoms. Patients with therapy refractory disease should receive interdisciplinary gastroenterological and surgical care from an early stage. Overall, the definition of therapy-refractory disease varies in the real clinical world, as a result of which patients often do not receive a recommendation for surgery as a serious alternative to intensification of drug therapy until it is too late. However, protracted therapy leads to increased morbidity in patients with severe disease [571]. For this reason, intensified conservative therapy should only be pursued for a limited period of time. This is confirmed by a study from Münster, in which, in retrospect, over $50 \%$ of patients would have preferred an earlier operation [572]. A more recent meta-analysis investigating the usefulness of third-line therapies in patients with severe chronic colitis showed that, although a short-term improvement may occur, in general, the necessity for colectomy is only delayed and the rate of side-effects increased [573].

\section{RECOMMENDATION 5.1.8}

Elective surgery can be performed at the request of the patient. In this instance, the risks of conservative therapeutic strategies are to be weighed up against the risks of surgery.

Evidence grade 4, recommendation strength 0 , strong consensus

\section{Background}

Surgery represents a good alternative to long years of conservative drug therapy. In the long term, despite all of its possible complications, restorative proctocolectomy with ileoanal pouch allows over $90 \%$ of patients to achieve a good quality of life. Patients who benefit especially from colectomy include those who continually feel ill and those who have poor adherence to drug therapy or fear of carcinoma [553, 554, 566].

A current case-control study comparing patients after pouch surgery versus patients under anti-TNF therapy detected no difference in quality of life (IBDQ). However, pouch patients had higher "QUALIs" and the overall costs for the health services were lower [574].

Intensive consultation with a visceral surgeon experienced in pouch surgery is a prerequisite for elective operation. Functional symptoms should be ruled out before making the decision in favour of surgery.

\section{RECOMMENDATION 5.1.9}

In children and adolescents with active colitis and growth impairment despite adequate therapy, following consultation with a paediatric gastroenterologist and after other causes have been ruled out, proctocolectomy with pouch-anal anastomosis should be performed.

Evidence grade 4, recommendation grade B, consensus 


\section{Background}

In children and adolescents with ulcerative colitis, growth impairment is much less frequent than in patients with Crohn's disease, and generally arises as a consequence of continual inflammatory activity or steroid therapy given for too long and at unnecessarily high doses, which must be avoided at all costs. Other causes of growth impairment (e.g. coeliac disease, growth hormone deficiency, constitutional growth retardation) must be ruled out prior to surgery. The drug therapy and indication for operation should be co-assessed by a paediatric gastroenterologist. Growth recovery after surgery is only to be expected in pre-pubertal children, or children in the early stages of puberty. Thus, although data are sparse, a strong recommendation appears justified [575].

\section{RECOMMENDATION 5.1.10}

In patients with an increased perioperative risk profile, depending on perioperative medication, proctocolectomy should be performed as a three-stage procedure.

Evidence grade 3, recommendation grade $B$, strong consensus

\section{Background}

The three-stage operative procedure involves 1) subtotal colectomy with end ileostoma, 2) rest-proctomucosectomy with ileoanal pouch creation und temporal loop ileostoma and 3) closure of the ileostoma. In patients with an increased perioperative risk profile, the three-stage operation is associated with a lower overall rate of complications than one- or two-stage procedures [576578]. Perioperative risk can be increased as a result of long-term steroid therapy, biologic therapy, immunosuppression, malnutrition (see recommendations 6.1.2-6.1.9), or concomitant illness [391, 579]. The intake of more than $20 \mathrm{mg}$ prednisolone over a duration of more than 6 weeks is associated with an increased risk of surgery-related complications. If possible, the steroid dose should be reduced prior to surgery, taking care to avoid the development of a steroid withdrawal syndrome. In children, a dose of $15 \mathrm{mg} / \mathrm{m}^{2}$ body surface (or $0.5 \mathrm{mg} / \mathrm{kg}$ ) is to be applied in analogy. A few studies have shown patients under anti-TNF therapy to have an increased risk of postoperative complications. Furthermore, three-stage procedures are more often performed in patients receiving TNF antibodies than in patients not under biologic therapy. Therefore, a potentially negative effect of these drugs on the risk of perioperative complications may be underestimated due to the choice of the least risky surgical procedure (i. e., colectomy with ileostomy) [580]. In the absence of prospective randomised studies, it remains unclear whether the increased rate of complications may rather be due to the presence of severe disease in patients then treated with biologics. In several case-control studies, an increased number of operative complications (e.g. pouch or anastomotic insufficiency) and septic complications was observed [391, 577, 581]. Two meta-analyses have been carried out on the subject, both of which concluded that, if all operations were included, TNF antibodies had no detectable effect on the perioperative complication risk $[582,583]$. In the later of these meta-analyses, however, a subanalysis of patients who had undergone pouch surgery revealed anti-TNF therapy to have a significant influence on the rates of both early and late pouch-specific complications. This correlation was confirmed in a contemporary analysis of a population of 2000 patients drawn from the databanks of US health insurance companies. In this collective, perioperative complications were significantly associated with the intake of infliximab up until 90 days prior to pouch surgery [584]. This is in line with the results of the two largest, statistically most valid case-control studies from two high-volume centres, both of which reported a significant association between perioperative complications of restorative proctocolectomy and anti-TNF therapy up to 3 months prior to surgery [577, 581].

The available data do not allow a conclusion to be drawn concerning the length of time that can be considered a safe interval between the last anti-TNF administration and planned surgery. From a pragmatic point of view, an interval of at least 4 weeks seems appropriate. A possible explanation for the contradictory results of the available analyses may be variations in the serum levels of anti-TNF antibodies in the respective study patients [585]. Further investigations are needed to determine whether preoperative trough level measurements may be a useful aid in determining when surgery can safely be performed.

Ongoing therapy with azathioprine is not associated with an increased risk of postoperative complications. A case series in paediatric patients detected no difference in the rate of postoperative complications with versus without preoperative therapy with calcineurin inhibitors [586].

\section{RECOMMENDATION 5.1.12}

In three-stage proctocolectomy, colectomy should be performed as far as the rectosigmoid junction.

Expert consensus, recommendation, strong consensus

\section{Background}

The high rate of complications under intensified drug therapy necessitates three-stage proctocolectomy, a procedure associated with fewer complications. Since removal of the rectum is the operative procedure which carries the highest risk and represents the greatest burden for the patient, in a first step, subtotal colectomy with end ileostoma should be performed. Care should be taken to remove as much inflammation-bearing colon as possible. As a rule, resection as far as the rectosigmoid junction fulfils these criteria and makes rest-proctectomy relatively simple from a technical point of view. A lower resection should be avoided, since it bears a distinctly increased risk of nerve injury in the subsequent operative stages. Closure of the rectal stump is performed either as a blind closure (Hartmann procedure), or through the creation of a mucous fistula by exteriorising the rectosigmoid remnant in the left lower abdomen. Utilisation of the latter variant avoids a possible insufficiency of the Hartmann stump and offers in addition the possibility of topical drug application (e.g. corticoids, mesalazine) via the sigmoid opening between the second and third stages of the operation. However, the partial retention of the sigma also 
results in more of the diseased bowel being left intact and the mucous fistula exteriorisation as a second stoma causes further weakening of the abdominal wall. In addition, the risk of stoma complications (e. g. stomal dehiscence) in these patients, whose sigma has been altered by severe inflammation, is considerable. This approach should therefore only be applied in exceptional cases [587-589].

\section{RECOMMENDATION 5.1.13}

In patients with a benign indication for surgery, resection should preserve the mesorectum, if technically possible, in order to keep the rate of complications, including nerve injuries, to a minimum.

Evidence grade 2, recommendation grade $B$, strong consensus

\section{Background}

The available data from an RCT, a case-control study and cohort studies indicate that preservation of the mesorectum leads to a better quality of life, an improved sphincter function and a lower rate of complications [590 - 592]. Since close rectal resection usually avoids the risk of nerve lesions, it would seem advisable to perform close rectal resection, as a rule, in patients with a benign indication for surgery, even though access can be more difficult, especially in the narrower male pelvis.

\section{RECOMMENDATION 5.1.14}

The rectal mucosa retained during ileoanal pouch surgery should be no longer than $2 \mathrm{~cm}$ in length.

Expert consensus, recommendation, strong consensus

\section{Background}

The last $2 \mathrm{~cm}$ oral of the linea dentata are functionally important and therefore of particular importance for patient quality of life. The anal transitional zone, which plays a major role in nocturnal continence, is situated within the last $2 \mathrm{~cm}$ oral of the linea dentata. When choosing the surgical approach, however, the postoperative risk of recurring/persistent proctitis must kept in mind.

Since the severity of disease and symptoms and the risk of relapse and/or persistent inflammation correlate exponentially with the length of the remnant rectal mucosa ("cuffitis"), the length of the spared rectal mucosa should not be more than $2 \mathrm{~cm}$. If inflammation arises within the remaining rectal mucosa, a topical therapy can be applied (see recommendation 5.2.6).

Although prospective randomised studies of adequate size comparing the techniques are lacking, the stapler anastomosis with retention of the anal transitional zone seems to have functional advantages over manual suturing. The evidence to date is inconclusive, a fact reflected in the two available, qualitatively mediocre meta-analyses [593, 594]. The smaller of these analyses, which concentrated explicitly on postoperative function and included 4 RCTs, found no advantage for either of the two methods. The larger analysis focused not only on complications, but also on func- tional and long-term outcomes, including data from 4183 patients from 21 studies, the majority of which were not randomised. The complications did not differ significantly, whereas functional parameters indicated significant disadvantages in the hand-sutured group in terms of nocturnal continence and the necessity to wear incontinence pads. However, these disadvantages were put into perspective when only the high-quality studies were evaluated. Regarding the development of dysplasia in the anal transition zone, a statistical trend was observed to the disadvantage of the stapler group. These findings appear logical, since, if stapled sutures are applied, more residual rectum mucosa remains, thus there is more potential for malignant degeneration. On the other hand, it must be kept in mind that even after mucosectomy with manual suturing, rectal mucosa remains in the form of islands of mucous membrane [595].

Every surgeon who carries out restorative proctocolectomy must be capable of manual transanal suturing, in case the stapler anastomosis should fail for technical reasons.

\section{RECOMMENDATION 5.1.15}

If the indication for surgery is intraepithelial neoplasia or manifest rectal carcinoma, complete mucosectomy with anastomosis at the linea dentata should be performed.

Evidence grade 3, recommendation grade B, strong consensus

\section{Background}

More than 50 cases of pouch carcinoma have been described in the literature. In the majority of these published cases, pouch carcinoma developed within the residual rectal mucosa [596, 597]. For pragmatic reasons, therefore, in patients with existing risks such as intraepithelial neoplasia or manifest rectal carcinoma, the entire rectal mucosa should be radically removed. Controversy remains as to whether mucosectomy should also be generally performed in patients with neoplasia of the colon. A retrospective cohort study from Canada evaluated data from 81 patients who had undergone restorative proctocolectomy with ileoanal pouch due to ulcerative colitis-associated dysplasia $(n=52)$ or carcinoma $(n=29)$. In this cohort, the incidence of pouch carcinoma or pouch dysplasia was found to be no higher in patients with stapled sutures $(n=59)$ than in patients with manual sutures [598]. Two patients with manual suturing developed a carcinoma in the rectal mucosa remnant or in the pouch, whereas in the stapler group, not a single patient developed carcinoma of the pouch or anastomosis. The authors conclude that mucosectomy with manual suturing has no prognostic advantage in patients undergoing surgery due to neoplasia who could equally be operated using stapled sutures, assuming that - as in this study - an average of only a little over $1 \mathrm{~cm}$ of remnant mucosa is spared. Finally, however, there are no data that clearly demonstrate oncological long-term benefits for mucosectomy with manual suturing. For this reason, the ECCO consensus guideline on surgery in ulcerative colitis also no longer contains an explicit general recommendation for mucosectomy in patients with colorectal neoplasia, unless neoplasia is located in the lower rectum 
[599]. Nevertheless, the largest available meta-analysis to investigate the occurrence of dysplasia in the anal transition zone shows a statistical trend toward a higher incidence in the stapler group. Thus, from a pragmatic perspective, the recommendation for mucosectomy still appears justified, at least in patients with rectal neoplasia [593].

\section{RECOMMENDATION 5.1.16}

Pouch surgery should only be carried out in specialised centres. Evidence grade 2, recommendation grade $B$, strong consensus

\section{Background}

Restorative proctocolectomy with ileum J-pouch/anal reconstruction is a complex and technically demanding procedure which requires long years of experience and a high degree of expertise, in both the pre- and postoperative phases [600]. Data from several studies provide evidence that mortality, morbidity and long-term pouch retention correlate significantly with the number of pouch operations carried out at the respective hospital [601 -603]. Minimum volume regulations have been in place for several years for procedures of comparable complexity (pancreas, oesophagus), stipulating, as a rule, a minimum of ten procedures annually. For ileoanal pouch surgery, the rate of pouch failure has been shown to drop significantly in centres performing more than 8 (versus $<8$ ), and more than 20 (versus $\leq 5$ ) operations per centre per year [555, 602]. For pragmatic reasons, therefore, the requirement for a minimum of 10 pouch procedures per year per centre would seem judicious.

\section{RECOMMENDATION 5.1.17}

Patients with chronic pouchitis or previous ulcerative colitisassociated carcinoma or intraepithelial neoplasia should undergo annual endoscopic surveillance.

Evidence grade 4, recommendation grade $B$, strong consensus

\section{Background}

Even after macroscopically complete mucosectomy, tiniest remnants of mucosa nevertheless pose a continuing risk [595]. These mucosal islands can be the source of intraepithelial neoplasia or carcinoma [597, 604]. For this reason, patients operated due to intraepithelial neoplasia or carcinoma require annual follow-up. Although there is no certain evidence that, in the long run, chronic pouchitis leads to malign degradation, annual postoperative endoscopic surveillance nonetheless seems sensible in this situation. In addition, this special group of patients requires close contact with the treating physician in order to improve quality of life with an existing pouch.

\section{RECOMMENDATION 5.1.18}

Colectomy with ileorectal anastomosis should only be recommended in special constellations, e. g., in patients wishing to have children.

Evidence grade 3, recommendation grade B, strong consensus

\section{Background}

Proctocolectomy with ileoanal pouch construction is associated with an increased rate of infertility in both women and men. It is unclear whether the fertility rate is reduced in men with benign indications for surgery who have undergone close rectal dissection. In fact, a large current register study from Denmark with 27379 patients shows that restorative proctocolectomy leads to a reduction in birth rates in females and an increase in birth rates in males [605]. In patients wishing to have children who have an indication for surgery, and in women additionally as a temporary solution until completion of family planning, subtotal colectomy with end ileostoma, or ileorectostomy, should be discussed as alternatives. The basic prerequisite for ileorectostomy, however, is that the residual rectum is by and large inflammation-free and thus suitable for anastomosis. Patients should be made fully aware that in approximately $50 \%$ of cases, restcolectomy is required, and that quality of life after ileorectostomy is not better than after pouch surgery. A large, recent study from France also showed that in $80 \%$ of patients who received therapy with immunosuppressants and biologics prior to operation (which is nowadays almost always the case if the surgical indication is a "therapy-refractory situation"), the ileorectostomy had to be abandoned within 10 years [606]. Moreover, patients who have undergone ileorectostomy often continue to suffer symptoms of urgency, one of the most distressing symptoms experienced by colitis patients even with an intact colon [607, 608]. Secondary rectum resection with proctectomy and ileal pouchanal anastomosis (IPAA) can be performed at a later time with results as good as in patients with primary pouch construction [609].

Due to the rarity of ileorectostomy in children, surgery in paediatric patients should be carried out at a centre specialised in visceral surgery, preferably in cooperation with paediatric surgeons. Postoperative care should always be provided by, or in close cooperation with, paediatric gastroenterologists.

\section{RECOMMENDATION 5.1.19}

In special cases, the continent "Kock" ileostoma can be offered as a possible alternative.

Evidence grade 4, recommendation grade 0 , strong consensus It should be carried out in centres specialised in this technique. Expert consensus, recommendation, strong consensus

\section{Background}

The Kock ileostoma, a continent ileostoma, involves a technically complicated operative procedure with high rates of both functional complications and surgical revision. The Kock ileostoma offers a better patient quality of life in comparison to the 
common, non-continent ileostoma, as well as improved physical comfort [610-612]. In patients who experience pouch failure and express a strong desire for the operation, conversion to the continent Kock ileostoma can be performed [613].

\section{RECOMMENDATION 5.1.20}

In patients who have undergone ileorectal anastomosis with sparing of the rectum, and patients with end ileostoma and blind rectal closure (Hartmann), the frequency of endoscopic monitoring should be chosen according to the initial indication for surgery. Monitoring colonoscopy should be consistent with the recommendation for general colonoscopy screening in ulcerative colitis.

Evidence grade 3, recommendation grade B, consensus

\section{Background}

In principle, as long as part of the rectum is spared, the risk of malignancy remains, and regular endoscopic monitoring with biopsies is indicated [614]. The ideal frequency of monitoring has not yet been adequately defined in studies. From a pragmatic perspective, the frequency recommended for ulcerative colitis monitoring seems appropriate (if there are risk factors for neoplastic lesions, see statement 2.31 and/or $>$ Table 6, otherwise according to the patient's symptoms and individual disease characteristics). A secondary restorative proctocolectomy with IPAA should generally be discussed with the patient, or possibly stump removal, if pouch creation is contraindicated or rejected by the patient. In any event, if there are classic risk factors for neoplastic changes (dysplasia or neoplasm at the time of the primary operation, PSC), these surgical options should be discussed in detail with the patient. Some published data indicate that, even in the presence of the above-mentioned risk factors, the incidence of new neoplasms in the longterm is still very low [615]. Nevertheless, the conflicting evidence in the literature suggests that regular monitoring after ileorectostomy or Hartmann stump construction is justified, not least because in comparison to the examination itself, which is unproblematic and associated only with a minimal risk, an overlooked neoplasm, albeit rare, has negative consequences on a much greater scale.

\section{RECOMMENDATION 5.1.21}

In elective surgery, laparoscopic restorative proctocolectomy is at least equal to the open surgical procedure, and in some respects, superior. In female patients wishing to have children, minimally invasive surgery should be favoured.

Evidence grade 3 , recommendation grade $B$, strong consensus

\section{Background}

Laparoscopic restorative proctocolectomy with IPAA is a procedure which can be safely performed in an adequately experienced centre. Studies to date show that, while both procedures have comparable rates of complications, the laparoscopic procedure has predominantly cosmetic advantages over open restorative proctocolectomy with IPAA [616, 617]. However, the laparoscopic operation takes longer to perform and therefore potentially entails higher procedural costs. A Cochrane review and two newer systematic reviews show that the minimally invasive procedure has several further advantages in the short term (wound infection rate, more rapid resumption of bowel function) [618-620].

A number of case-control and cohort studies suggest that female fertility is less impaired by laparoscopic surgery, presumably because adhesions occur less frequently [621-624]. Whether this reduction in postoperative adhesions also leads to fewer episodes of ileus remains to be determined [625].

Since, in principle, the laparoscopic operation is at least as good as open surgery, has clear cosmetic advantages, and also better preserves female fertility, it should be the method of choice in women of childbearing age.

Several case-control studies have shown that the advantages associated with the laparoscopic technique in elective surgery apply similarly when surgery is performed as a matter of urgency or emergency [626-628]. However, since all of these studies, without exception, were carried out in specialised centres, whereas the majority of emergency operations are not performed in such centres, a general recommendation in favour of laparoscopic access cannot be given at this time.

\section{RECOMMENDATION 5.1.22}

Patients with indeterminate colitis without anorectal fistula formation, and therefore without the associated indication for surgery, can be offered restorative proctocolectomy after being given a detailed explanation of the procedure and its associated risks.

Evidence grade 3, recommendation grade 0 , strong consensus

\section{Background}

In approximately $7 \%$ of patients with colitis, it is not possible to make an exact differentiation between ulcerative colitis and Crohn's disease [629]. There are reports in the literature describing poorer long-term results after proctocolectomy with IPAA in patients with indeterminate colitis. However, these data are in contrast to other accounts in which the outcomes of patients with indeterminate colitis have been found to be no worse than those of patients with ulcerative colitis $[630,631]$. A secondary diagnosis of Crohn's disease after restorative proctocolectomy with IPAA is frequently associated with complications, and in particular with an increased rate of pouch failure. However, this is put into perspective in the subgroup of patients who had colitis without fistulae and without small bowel involvement prior to surgery [632]. Despite these contradictory data, after thorough discussion with the patient, restorative proctocolectomy with IPAA can be performed.

The proportion of children and adolescents with indeterminate colitis lies by up to $22 \%$ [633]. For the most part, the diagnostic classification of the disease as ulcerative colitis or Crohn's disease will succeed during the course of the disease. Therefore, in children requiring ulcerative colitis surgery, it is advisable to carry out a three-stage procedure. 


\subsection{Pouchitis}

\section{RECOMMENDATION 5.2.1}

The diagnosis of pouchitis should be made on the basis of clinical, endoscopic and histologic parameters.

Evidence grade 5, recommendation grade B, strong consensus

\section{Background}

Pouchitis is defined as inflammation of the pouch after operative complications or other secondary causes have been excluded. Different forms of pouchitis should be differentiated according to the temporal progression of disease, as acute, acute-relapsing or chronic pouchitis. Further classification can be made according to the clinical course: antibiotic-sensitive or -refractory acute pouchitis, relapsing or chronic pouchitis (e. g., >3 months) [634, 635].

The diagnosis of acute pouchitis is made on the basis of clinical symptoms (stool frequency, bleeding, fever, pain), taking into account endoscopic (redness, oedema, erosion, ulceration, spontaneous haemorrhage, fibrin deposits) and histological findings (ulceration, crypt abscesses, inflammatory cell infiltration), and the clinical, in particular rectal-digital, examination [636]. Acute-relapsing pouchitis is characterised by repeated flares of inflammation. The diagnosis of chronic pouchitis describes an inflammatory reaction in the pouch, the symptoms and endoscopic/histologic findings of which persist for more than 3 months. The risk of acute pouchitis after restorative proctocolectomy with IPAA in patients with ulcerative colitis lies at approximately $30 \%$ in the first two years after surgery. This risk increases to approximately $50 \%$ during the course of long-term follow-up [637-643]. In around $5 \%$ of patients with pouchitis, the initial acute disease becomes chronic over time. Existing extraintestinal manifestations of ulcerative colitis are risk factors for pouchitis, especially PSC [643-647]. Also associated with an increased rate of pouchitis are persistently high inflammatory activity and preoperative backwash ileitis. Parameters for the diagnosis of acute pouchitis are combined in the Pouchitis Disease Activity Index (PDAI) [636]. If there are symptoms of pouchitis, but endoscopy, imaging tests and histologic examination fail to identify their cause, assuming other diseases have been ruled out, the diagnosis of irritable pouch syndrome can be made [648].

\section{RECOMMENDATION 5.2.2}

In patients with chronic pouchitis, Crohn's disease, surgical complications and infection should be ruled out.

Evidence grade 5, recommendation grade B, strong consensus

\section{Background}

When diagnosing pouchitis, it is recommended to perform examinations to rule out secondary forms of pouchitis. To rule out surgical causes, computer tomography, magnetic resonance imaging, contrast enema and endosonography are necessary and suitable [649-652]. These procedures may need to be repeated in the course of follow-up. The presence of Crohn's disease, fistula or abscess development, anastomotic insufficiency, ischaemia or opportunistic infection should be ruled out. In addition to histologic evidence, involvement of the pre-pouch ileum segment (the afferent limb at the pouch inlet) suggests a diagnosis of Crohn's disease.

\section{RECOMMENDATION 5.2.3}

Ciprofloxacin or metronidazole should be administered as primary therapy for acute pouchitis.

Evidence grade 1, recommendation grade A, strong consensus If monotherapy fails, combination therapy can be used.

Evidence grade 3, recommendation grade 0 , consensus Antibiotic-refractory disease forms should be treated with oral or topical budesonide.

Evidence grade 3, recommendation grade B, strong consensus Further options for therapy can include infliximab, adalimumab, vedolizumab, ustekinumab or calcineurin inhibitors, amongst others.

Expert consensus, recommendation open, consensus

\section{Background}

A number of randomised controlled studies have focused on drug therapies in pouchitis [653-656]. The recommendation for therapy of acute pouchitis with antibiotics (metronidazole, ciprofloxacin) is backed up by several studies, albeit small [657-660]. There is a suggestion that ciprofloxacin may be superior to metronidazole therapy [656]. A combination therapy is also possible $[661,662]$

Since there is insufficient evidence on which to base a recommendation for the dosage and duration of antibiotic therapy, these must be tested on an individual basis. The usual dose is $2 \times 250 \mathrm{mg}$ up to $2 \times 500 \mathrm{mg} / \mathrm{d}$ ciprofloxacin p. o. for 2 weeks or $2-3 \times 400 \mathrm{mg}$ metronidazole p. o. In patients intolerant to oral metronidazole, topical metronidazole therapy (suppositories) may be used as an alternative [663]. No recommendation can be given regarding rifaximin therapy [664].

Patients with antibiotic-refractory pouchitis may benefit from topical budesonide therapy [665]. While topical budesonide is as effective as a topical metronidazole therapy, it seems to be somewhat better tolerated [666].

Therapy with TNF antagonists and/or immunosuppressants is possible. In a recently-published review, 19 publications with a total of 192 patients were analysed in this respect [667]. Indications for anti-TNF therapy were antibiotic-refractory, fistulising disease and inflammatory, stenosing forms of pouchitis. Based on the 3 largest studies included in the analysis $(n=87)$, the combined rate of partial and complete response to infliximab therapy was $84 \%-88 \%$ after $6-10$ weeks and $45 \%-58 \%$ after 52 weeks [667].

In a retrospective Canadian study, 152 patients with therapyresistant pouchitis were analysed, of whom 42 were treated with infliximab. Post-induction response was achieved in $74 \%$ and 
long-term response in 62.6\%. Mean PDAI and CRP values showed a statistically significant decrease under therapy [668].

The effect of adalimumab was demonstrated in a case series including 48 patients. The combined rate of partial and complete response was $71 \%$ and $54 \%$ after median observation times of 8 and 25 weeks, respectively [669]. Among patients with therapy-refractive pouchitis and infliximab therapy failure, it was possible to avert the necessity for permanent ileostoma at week 52 in $50 \%$ of cases through second-line therapy with adalimumab [670].

Further therapeutic options include vedolizumab, ustekinumab or calcineurin inhibitors. However, the pertinent studies were carried out in patients with acute, antibiotic-refractory pouchitis and consist of retrospective data and case series with heterogeneous patient collectives [653, 671 -673].

\section{RECOMMENDATION 5.2.4}

In patients with frequently relapsing or chronic pouchitis, intermittent monotherapy or a combined antibiotic therapy with ciprofloxacin and/or metronidazole should be given. Evidence grade 3, recommendation grade $B$, strong consensus Antibiotic-refractory pouchitis should be treated with oral or local budesonide.

Evidence grade 3, recommendation grade B, consensus

Further therapeutic options include infliximab, adalimumab, vedolizumab, ustekinumab, rifaximin, calcineurin inhibitors or alicaforsen.

Evidence grade 4, recommendation grade 0, consensus

\section{Background}

Patients who fail to respond to metronidazole or ciprofloxacin monotherapy can be treated with an oral combination therapy of ciprofloxacin and metronidazole, or an oral combination therapy of ciprofloxacin and rifaximin [657, 662]. However, the supporting evidence is weak.

In a recent review, the effectiveness of different drug therapies in chronic pouchitis after IPAA in UC patients was evaluated [653]. The review included 21 manuscripts. The results showed that antibiotics led to remission in $74 \%$ of cases ( $95 \% \mathrm{Cl}: 56-93 \%$ ), $(\mathrm{P}<0.001)$, whereas TNF antibodies achieved remission rates of $53 \%$ (95\% Cl:30 - $76 \%)$, ( $<<0.001)$. Therapies with steroids, bismuth, elemental diet or tacrolimus also achieved remission, although non-significantly. Local therapy with tacrolimus is described in single case reports. Evidence concerning the use of FMT in pouchitis is currently insufficient to warrant recommendation, and failed to show induction of remission [674, 675]. Further therapeutic options include infliximab, adalimumab, vedolizumab, ustekinumab, rifaximin, calcineurin inhibitors or alicaforsen [653, 667, 671-673, 676-679].

With regard to the bacterial formulation VSL\#3, there are a number of older studies that have shown efficacy in the treatment of pouchitis. A pooled analysis of two studies (76 participants) suggests that VSL\#3 is more effective than placebo in remission maintenance [680 -682]. Eighty-five per cent (34/40) of patients under VSL\#3 were able to maintain remission for a period of 9 12 months, in contrast to the placebo group, in which only $3 \%$ (1/36) remained in remission (RR $20.2495 \% \mathrm{Cl} 4.28$ to 95.81 ). However, a GRADE analysis indicated that the evidence was of low quality low due to the small number of cases [656].

Perianal inflammation and irritation present considerable problems for many patients who have undergone pouch surgery. Diagnosis can often be made on the basis of the past medical history, examination and if necessary, a smear test. Measures which can contribute to wound healing include rinsing after defaecation or the application of moist tissues free from alcohol, perfume or colourings, optimal skin care, e.g., with panthenol or zinc, nutritional changes, and possibly the use of cholestyramine.

\section{RECOMMENDATION 5.2.5}

If risk factors are present, an endoscopic surveillance examination should be carried out annually. Otherwise, pouchoscopy should be performed if symptoms occur, or every 2 years at a minimum.

Expert consensus, recommendation, consensus

\section{Background}

This recommendation is not backed up by specific studies in the literature. Based on experience of surveillance strategies prior to restorative proctocolectomy with IPAA, annual endoscopic surveillance would seem appropriate, even though the pouch carcinoma is a rare entity [683].

Whether monitoring can be performed less frequently in patients who undergo pouch surgery for reasons other than malignancy is unclear. As a rule, patients who develop a pouch carcinoma are those in whom colon or rectum carcinoma was the indication for restorative proctocolectomy with IPAA. Pouch carcinomas usually occur in the remnant mucosa of the anorectal transition. Thus, from a pragmatic perspective, in patients with a carcinoma of the lower rectum, restorative proctocolectomy should preferably be performed with a complete mucosectomy (see also statement 5.1.15) [604, 684].

\section{RECOMMENDATION 5.2.6}

In cuffitis, topical mesalazine therapy should initially be attempted.

Evidence grade 3, recommendation grade B, strong consensus In patients with therapy-refractory inflammation, endoscopic mucosectomy or rest-proctectomy can be considered.

Expert consensus, recommendation, consensus

\section{Background}

Cuffitis can cause pouch dysfunction with symptoms which can imitate pouchitis or irritable pouch syndrome (IPS), especially in patients who have undergone double-stapled IPAA.

In contrast to IPS (which may occur coincidentally), perianal bleeding is a characteristic sign of cuffitis. Diagnosis can be 
made by endoscopy at the hands of an experienced examiner and histological evaluation of the epithelial cuff between the linea dentata and the pouch-anal anastomosis [685].

In an open-label study including 14 consecutive patients with cuffitis, the effect of mesalazine $500 \mathrm{mg}$ suppositories was examined in a blinded manner. Compared to placebo, mesalazine led to a significant reduction in the total cuffitis index, as well as in the symptom, endoscopy and histology subscores. Ninety-two per cent of patients with bloody stools and $70 \%$ of patients with arthralgia (a characteristic clinical symptom of cuffitis) responded to topical mesalazine therapy. There were no reports of systemic or topical side-effects [686].

\section{RECOMMENDATION 5.2.7}

Patients with irritable pouch syndrome can be treated in the same way as patients with irritable bowel syndrome.

Expert consensus, recommendation open, consensus

\section{Background}

Unlike the therapeutic approach in irritable bowel syndrome (IBS), IPS management has not been defined in a treatment algorithm. Analogous to patients with irritable bowel syndrome, patients with IPS can be treated with symptomatic therapies, e. g., dietary modifications, dietary fibres, loperamide, anticonvulsant drugs and antidepressants [648,687].

\section{Nutrition and complementary therapies}

\subsection{Nutrition in the aetiology and prevention of ulcerative colitis}

\section{RECOMMENDATION 6.1.1}

With the exception of breastfeeding, there are no nutritional factors which could be recommended to reduce the risk of ulcerative colitis. Breastfeeding should be continued for at least six months.

Evidence grade 3, recommendation grade $B$, consensus

\section{Background}

The observation that ulcerative colitis was extremely rare until the end of the $1950 \mathrm{~s}$, and has since then substantially increased in all western industrial nations, led to the presumption that nutritional changes represent a possible cause for the increased incidence of the disease. Retrospective studies have suggested a possible complicity of various factors, including the post-war increase in consumption of refined carbohydrates, chemically processed fats, reduced intake of dietary fibres, and an allergic reaction to baker's yeast. More recent prospective studies showed a possible association between ulcerative colitis development and an increased consumption of animal protein [688] as well as saturated fatty acids and trans fats $[682,689]$. On the other hand, a significant inverse relationship with ulcerative colitis occurrence has been demonstrated with respect to the daily intake of vegetables, omega-3 fatty acids [690] and vitamin D [691].

On the evidence of two systematic reviews dating from 2004 and 2009 , only breastfeeding has been proven to result in a significant reduction ( $23 \%$ ) in the risk of later ulcerative colitis development [692, 693]. This was corroborated by two later cohort studies from New Zealand [694] and Denmark [695]. Breastfeeding should be continued for at least 6 months, and if possible, longer [696].

\section{Malnutrition}

\section{RECOMMENDATION 6.1.2}

Patients with ulcerative colitis have an increased risk for malnutrition. Accordingly, they should be assessed with regard to malnutrition at first diagnosis and during follow-up. Evidence grade 2, recommendation grade B, strong consensus

\section{Background}

In many patients with ulcerative colitis, complications due to malnutrition can be a greater source of weakness than the underlying inflammatory process itself. Therefore, the prevention and treatment of malnutrition are essential elements of IBD therapy.

The prevalence of malnutrition in adults with active ulcerative colitis is $7.2 \%$ compared with $1.8 \%$ in healthy controls, representing a five-fold increased risk (OR 5.57; $95 \%-K I$ : 5.29-5.86). The coherent risk of complications (severe disease, longer hospitalisation) and the treatment costs are also increased [697].

Up to $85 \%$ of children with IBD show signs of malnutrition (Crohn's disease > ulcerative colitis), of whom 15-40\% have retarded growth [696]. In approximately $25-80 \%$, albumin levels are diminished. Depending on the activity and duration of disease, $20-85 \%$ of patients show a negative nitrogen balance. The main cause of total body protein depletion appears to be the excessive loss of protein through the inflamed bowel mucosa, determinable by measurement of faecal $\alpha_{1}$-antitrypsin. Protein malabsorption could be an additional contributor. Another possible cause of the negative nitrogen balance is the catabolic effects of drugs such as antibiotics and steroids [698].

\section{RECOMMENDATION 6.1.3}

Patients with ulcerative colitis have an increased risk of micronutrient deficiency. They should therefore be assessed for micronutrient deficiencies initially and during follow-up whenever there are clinical symptoms or an increased risk of deficiency.

Evidence grade 2, recommendation grade B, strong consensus

\section{Background}

Depending on the patient collective, disease activity and the choice of biomarker and cut-off point, iron deficiency occurs in $30-70 \%$, 25-OH-vitamin D deficiency in $40-60 \%$, folic acid 
deficiency in 5-10\%, zinc deficiency in $20-30 \%$ and selenium deficiency in 20-30\% of patients with ulcerative colitis. In contrast, vitamin $B_{12}$ deficiency has been described only after IAP creation [698 - 702]. While the aetiology of vitamin $B_{12}$ deficiency in patients with IAP is multifactorial, a diminished resorptive capacity, as well as bacterial overgrowth, which occurs almost universally as a result of IAP, are suspected to play a role [703].

\section{Nutritional and supplementation therapies}

\section{RECOMMENDATION 6.1.4}

Special enteral nutrition therapy and/or total parenteral nutrition should not be administered as a primary therapy for remission induction in patients with ulcerative colitis. Expert consensus, recommendation, strong consensus

\section{Background}

In contrast to Crohn's disease, there is little or no evidence demonstrating an influence of nutritional therapy (sip feeds, enteral and parenteral feeding) on disease activity in acute or chronically active ulcerative colitis [704]. In IBD, as in other diseases, the principles of a stepwise approach to nutritional therapy are applicable including nutritional consultation, complementary feeding in the form of sip feeds, tube feeding, and parenteral nutrition [704]. When treating children, it is important to bear in mind that nutritional/dietary counselling alone is not an effective treatment for malnutrition and/ or specific deficiencies, or for growth retardation [704].

Enteral nutrition (total or partial) should be given as supplementary therapy, in order to ensure an adequate supply of nutrients during acute phases of inflammation, especially in patients with signs of existing malnutrition or at high risk for nutritional deficiencies [345].

Nevertheless, especially in patients with fulminant ulcerative colitis, there may be an indication for total parenteral or enteral nutrition with a standard regimen to secure an adequate supply of nutrients. An influence of specific substrates such as omega-3 fatty acids, glutamine or butyrate on disease activity in patients with ulcerative colitis has not been demonstrated [705-709].

Assuming that no weight loss occurs, energy requirements are between 25 and $30 \mathrm{kcal} / \mathrm{kg}$ BW/day [710]. As a result of the catabolic metabolic state resulting from inflammation, and due also to intestinal protein loss, protein requirements are increased to 1 $1.5 \mathrm{~g} / \mathrm{kg} \mathrm{BW} /$ day, and in patients with sepsis or very severe malnutrition, up to $2 \mathrm{~g} / \mathrm{kg}$ BW/day [710]. Total parenteral nutrition (TPN) alone should only be administered to correct severe malnutrition, especially prior to surgery, or if enteral feeding is either impracticable or unable to provide sufficient calories in patients with complicated disease [711, 712].

\section{RECOMMENDATION 6.1.5}

A special diet or nutritional therapy should not be recommended for maintenance of remission.

Expert consensus, recommendation, strong consensus

\section{Background}

While small, uncontrolled studies of nutritional supplements enriched with omega-3 fatty acids have shown a positive effect on remission maintenance in patients with Crohn's disease, a general therapeutic recommendation for patients with ulcerative colitis can currently not be derived from these findings [713]. The same is true for the low-sulphite diet suggested by Roediger in the mid-90 s for patients with ulcerative colitis, which prescribes the avoidance of foods rich in sulphurous amino-acids [709, 714].

\section{RECOMMENDATION 6.1.6}

An isolated micronutrient deficiency should be treated by appropriate oral or parenteral supplementation. Routine substitution of vitamins or minerals in patients with ulcerative colitis is not appropriate.

Expert consensus, recommendation, strong consensus

\section{Background}

In patients with confirmed micronutrient deficits, specific vitamins and/or minerals should be substituted according to the appropriate recommendations $[702,715,716]$. During the remission phase, nutritional substitution can primarily be administered orally. In the case of intolerance to oral preparations (e. g., iron) or insufficient resorption (iron, vitamin $B_{12}$ ), especially in the presence of substantial inflammatory activity, parenteral substitution is required.

Iron should always be supplemented if there is manifest anaemia. Iron deficiency without manifest anaemia requires an individualised approach. The choice of timing and type of therapy depends on symptoms, aetiology, severity, dynamics of haemoglobin depletion, comorbidities and risks of therapy [717].

The therapeutic goals of iron substitution in patients with iron deficiency anaemia are to increase the haemoglobin level by more than $2 \mathrm{mg} / \mathrm{dL}$ or up to normal levels within 4 weeks, and to increase serum ferritin levels to $>100 \mu \mathrm{g} / \mathrm{L}$. The universally recommended calculation of iron requirements using the Ganzoni formula (total iron deficit $(\mathrm{mg})=[$ target $\mathrm{Hb}-$ actual $\mathrm{Hb}(\mathrm{g} / \mathrm{dL})] \times$ body weight $(\mathrm{kg}) \times 2.4+$ iron stores $(\mathrm{mg})$ ) is inconvenient in routine practice and underestimates iron requirements, at least in IBD patients [718]. The recently-published "FERGIcor" tool has proved to be simpler and more effective [719].

In principle, iron can be substituted in either of two ways; by oral administration of suitable iron(II, III) compounds or intravenous application of iron(III) complexes [718].

Mild iron deficiency anaemia ( $\mathrm{Hb} 11-13 \mathrm{~g} / \mathrm{dL}$ ) can initially be treated with oral iron therapy of approx. $100 \mathrm{mg} /$ day for 4 weeks. Patients who show intolerance to oral therapy or an inadequate haemoglobin response, i. e., an increase of less than $2 \mathrm{~g} / \mathrm{dL}$, should be switched to intravenous iron therapy in good time; since resorption of oral iron is limited, patients with severe anaemia should always receive intravenous iron substitution, with the aim of normalising haemoglobin values and replenishing iron stores. Absolute indications for intravenous iron therapy are $[717,718]$ : 
- Severe anaemia $(\mathrm{Hb}<10 \mathrm{~g} / \mathrm{dL})$

- Intolerance of oral iron or insufficient haemoglobin increase during the first 2 weeks

- Severe disease activity

- Adjuvant therapy with erythropoietin-stimulating agents

- Patient preference

\section{Therapy of zinc deficiency}

Large quantities of zinc are lost in diarrhoea (stool volume $>300 \mathrm{~g} /$ d) or stoma output, whereby some $12 \mathrm{mg}$ elemental zinc may be lost per litre of stoma output. This is considerably more than the normal daily requirement for zinc and more than regular mineral supplements contain [720,721]. Patients with zinc deficiency should receive 30 to $45 \mathrm{mg}$ oral zinc histidine or zinc gluconate (taken approximately 1 hour before breakfast) [722]. As zinc interferes with the intestinal absorption of iron and especially copper, oral supplementation should not be continued for longer than 2 3 weeks [723]. If longer supplementation is necessary, $1 \mathrm{mg}$ elementary copper should be substituted along with every 8 $15 \mathrm{mg}$ elementary zinc. If there is insufficient response, as is frequently the case, zinc should be substituted parenterally (e. g. up to $5 \mathrm{mg} /$ day zinc aspartate as mono-injection or infusion additive) $[721,724]$.

\section{Therapy of vitamin $\mathrm{B}_{12}$ deficiency}

Since only $1-3 \%$ of cyanocobalamin is resorbed, parenteral application is generally preferred. Unfortunately, therapy recommendations concerning the dosing and application of $\mathrm{B}_{12}$ substitution are still inconsistent and the dosages mostly underestimated. Two medicinal vitamin $B_{12}$ preparations are currently available: cyanocobalamin and hydroxocobalamin. When calculating the dosage, it should be kept in mind that clinical symptoms occur only after the body's vitamin $B_{12}$ stores $(4-5 \mathrm{mg}$ ) are reduced to 5 to $10 \%$ of normal levels. Therefore, the goal of therapy must be to compensate this deficit. In order to refill the empty body stores as rapidly as possible, the following treatment is recommended: In the first week, on 5 days, $1000 \mu \mathrm{g}$ hydroxocobalamin i. m./s.c. (of which approx. $45 \%$, i. e. $450 \mu \mathrm{g}$, will be resorbed - compared with only $16 \%$ of cyanocobalamin). Over the following month, this is followed by weekly injections of $1000 \mu \mathrm{g}$ (alternatively, $500 \mu \mathrm{g}$ hydroxocobalamin daily on 5 days per week for one month). The measurement of homocystein or MMA levels has proved useful in the monitoring of vitamin therapy [718].

\section{Therapy of vitamin $\mathrm{D}$ deficiency}

Recommendations for target levels of $25-\mathrm{OH}$-vitamin $\mathrm{D}$ and for supplementation dosage vary [725]. The Endocrine Society recommends a daily vitamin D intake of 1500 - 2000 IE per day in adults, targeting a vitamin D level of $>75 \mathrm{nmol} / \mathrm{L}$ (>30 ng/mL). In certain situations, such as obesity or concomitant intake of anticonvulsive or glucocorticoid therapy, this dose can be doubled or even trebled. Especially under glucocorticoid therapy, vitamin D levels can fall, and should therefore either be monitored, or 1000 IE $25-0 H$-vitamin D should be supplemented daily [726, 727]. Evidence-based recommendations concerning target levels, dosage and duration of vitamin D supplementation in patients with IBD are lacking. Garg et al. recommend $1000-5000 \mathrm{IU} /$ day depending on serum levels, with a multiplying factor of 1.5-20 in patients with Crohn's disease of the small bowel and/or obesity. Levels of $>30 \mathrm{ng} / \mathrm{mL}$ (75 mmol/L) 25-OHvitamin $\mathrm{D}$ should be sustained. In order to normalise $25-\mathrm{OH}$ levels as quickly as possible (within 2 weeks), Van Groningen et al. suggest an initial repletion dose $(\mathrm{IU})=40 \times\left(75\right.$-serum-25- $\left.\mathrm{OHD}_{3}\right) \times \mathrm{BW}$ [728]. The calculated dose is to be administered on 3 weekdays (Mon./Wed./ Fri.) in portions of $20000 \mathrm{IU}$ each, followed by a weekly maintenance dose of $20000 \mathrm{IU}$. In general, cholecalciferol (vitamin D3) should be preferred over ergocalciferol (vitamin D2) due to its better bioavailability [729].

An intake of up to 4000 IE vitamin D3 per day does not necessitate monitoring of vitamin $\mathrm{D}$ levels, as a rule. Vitamin $\mathrm{D}$ toxicity only begins to occur if levels $>150 \mathrm{ng} / \mathrm{mL}$ (> $375 \mathrm{nmol} / \mathrm{L}$ ) are sustained $[730,731]$.

\section{Surgical aspects of nutrition in UC}

\section{RECOMMENDATION 6.1.7}

Before elective surgery, patients with severe malnutrition (BMI $<19$, weight loss $>10 \%$ in the last 6 months prior to surgery and/or serum albumin $<30 \mathrm{~g} / \mathrm{L}$, marked cachexia) should be treated with targeted nutrition therapy for at least 7 days. Expert consensus, recommendation, strong consensus

\section{Background}

Patients with severe malnutrition have a significantly higher risk for postoperative complications [712, 732, 733]. Prior parenteral nutrition was shown to exert a positive effect only if begun at least 5 days prior to surgery [734]. Targeted preoperative nutrition therapy before elective surgery should therefore be administered over at least 7 days. Details on the administration of nutritional therapy are given in paragraph 6.1.4. Administration of TPN as a perioperative measure can improve nutrition status and thus reduce postoperative complications [735]. Severe malnutrition is present when one or more of the following criteria are met:

- Weight loss $>10-15 \%$ within 6 months

- $\mathrm{BMI}<18.5 \mathrm{~kg} / \mathrm{m}^{2}$

- Serum albumin $<30 \mathrm{~g} / \mathrm{L}$ (without impairment of liver or kidney function)

If preoperative nutrition therapy is indicated, enteral intake via sip feeds or tube feeding should be preferred. If possible, in order to avoid nosocomial infections, enteral nutrition should be administered prior to hospitalisation. Parenteral nutrition therapy is generally reserved for patients with severe colon inflammation and intestinal intolerance [736-738].

RECOMMENDATION 6.1.8

Ulcerative colitis patients with high-output jejuno- or ileostomy should be closely monitored with regard to water and electrolyte levels.

Expert consensus, Recommendation, strong consensus 


\section{Background}

The goal of nutrition therapy after ileostomy (depending on the remnant bowel) is to prevent the loss of water and electrolytes and to avoid irritation of the stoma by chemicals from ingested foods. Only after approximately eight (and sometimes even up to twelve) weeks does the consistency of the stool stabilise. However, it remains liquid or soft. In the adaptation phase (see above), clogging foods such as potatoes, rice, oatmeal or bananas can have a positive effect. Foods which swell by binding liquid, and preparations such as pectin, which increase the viscosity of the stool, may also be useful.

The basis of nutrition therapy in patients with ileostomy, during the gradual reintroduction of liquid and solid foods and the adaption phase, is the adequate intake of fluid and salt. It is important for the patient with ileostomy to know that every intake of food will lead to the emptying of the stoma.

The fluid requirement is approximately 3 litres per day. A gauge of sufficient fluid intake is a urine volume of at least 1 litre per day (urine volume should be regularly monitored). The recommended intake of sodium chloride is $6-9 \mathrm{~g}$ per day (e. g., salted meat and vegetable broths). Dietary fibre intake should be approximately 25 - $30 \mathrm{~g}$ per day [739].

\section{RECOMMENDATION 6.1.9}

Patients with a pouch should regularly be monitored for possible deficiencies of vitamin D, vitamin B12 and iron.

Expert consensus, recommendation, strong consensus

\section{Background}

Anaemia due to iron and/or vitamin $B_{12}$ deficiency is one of the most frequent extraintestinal manifestations after IAP [740, 741]. Depending on definition and methods, iron deficiency has been reported in $20-56 \%$ [703, 742] (pouchitis 77\%) [700] of patients, $B_{12}$ deficiency in $25 \%$, vitamin D deficiency $(<21 \mathrm{ng} / \mathrm{mL})$ in $22 \%$ and vitamin D insufficiency $(<31 \mathrm{ng} / \mathrm{mL})$ in $70 \%$ [743]. Deficiencies of vitamin $E$ and/or A occur in up to $5 \%$ of patients in the context of fat malabsorption [744]. In patients with longer-term steatorrhoea, vitamin $\mathrm{A}$ and $\mathrm{E}$ levels should therefore be determined at least once. Data on folic acid status are incongruent and do not allow a recommendation to be made [740].

\subsection{Complementary therapies}

Preamble

There is no generally accepted definition of complementary and alternative therapies. The concept of complementary therapy is that the treatment methods applied are ancillary, i. e., supplementary to standard methods. Therapies which preclude the use of standard methods are described as alternative therapies. Unconventional therapies are all treatment methods which are not considered to be approved, accepted or scientifically evaluated.

These guidelines will apply the classification of Complementary and Alternative Medicine (CAM) according to Wieland et al. [745] for the Cochrane Collaboration. This classification differentiates five categories: (1) mind-body methods, (2) holistic (integra- ted) therapies, (3) herbal therapies, (4) manual and body-related therapies and (5) energy medicine.

As a rule, the terminology "alternative therapies" does not reflect how these methods are commonly used, since complementary/naturopathic therapies are not intended to be substitutes for conventional therapies. Rather than differentiating between alternative and complementary medicine, the AngloAmerican literature uses the collective term "complementary and alternative medicine" (CAM), which has now found wide acceptance internationally. To describe the combined application of conventional and complementary methods, more recent literature has adopted the term "integrative medicine"

\section{RECOMMENDATION 6.2.1}

The use of alternative therapies instead of a clinically proven therapy should be rejected.

Expert consensus, strong recommendation, strong consensus

\section{Background}

Since alternative therapies are intended to replace a clinically proven therapy, these are to be rejected, due to the lack of evidence to support an equipotential effect (thus, phytotherapeutics and other immunomodulatory substances with confirmed equipotency to a standard therapy are not alternative therapies (see below)). Complementary therapies, which are applied adjunctively to standard therapies, should be carried out in cooperation with the treating physicians and can reinforce the standard therapy. The wide public interest in alternative and complementary approaches justifies the additional evaluation of these methods, which include: traditional Chinese medicine (TCM) including acupuncture, anthroposophic medicine, aromatherapy, Ayurveda, homeopathy, "immunomodulative" therapies, manual therapies (osteopathy, massage, etc.), mind/body techniques, nutritional supplements, naturopathy/herbalism, Qi Gong, and reiki.

\section{RECOMMENDATION 6.2.2}

Naturopathic and complementary methods should be assessed according to the criteria of evidence-based medicine. Expert consensus, strong recommendation, strong consensus

\section{Background}

According to the CONSORT Consensus, the evaluation of an evidence-based medicine (EBM)- hierarchisation is determined by the method and the question posed. This should be considered when evaluating the literature on complementary medicine. Several complementary therapies (so-called complex individual therapeutic interventions) depend on the salutogenic potential of the patient and require a non-blinded physician-patient relationship, since corrective interactions are impossible if doctor and patient are blinded. Salutogenic therapies are usually characterised by learning and regulative processes analogue to those which take place between teacher and pupil, which are dialogic in nature. The 
randomised controlled trial (RCT) study design is therefore not always practicable. This must be borne in mind when hierarchising evidence-based medicine. Moreover, complementary and alternative therapies are usually complex techniques which cannot be substantiated by proof of the effect of any one factor, but require systematic data collection. In this case, outcome studies comparing cohorts are the appropriate method of evaluation and for the most part, their results are on a par with those of an RCT.

\section{RECOMMENDATION 6.2.3}

Patients should be questioned regarding the use of complementary therapies. Treating physicians should discuss with them their reasons for adopting complementary treatment methods. Expert consensus, strong recommendation, strong consensus

\section{Background}

Numerous studies have reported that at least half (31\% to $68 \%$ ) of patients with IBD use complementary therapeutic techniques [746 - 752]. In children with IBD, the use of CAM is no less frequent than in adults.

For the German population, a representative study indicated a $52.9 \%$ rate of uptake among patients with IBD [752]. In IBD patients, the most commonly used complementary methods are homeopathy, phytotherapy, traditional Chinese medicine including acupuncture, diet therapies, vitamins and nutritional supplements. Predictors for the adoption of complementary methods are a higher degree of education, a whole-food diet and a total cumulative oral corticoid intake of more than $10 \mathrm{mg}$. A higher body mass index (BMI) was negatively associated with the use of CAM.

Three in four patients indicated having had experience of more than one complementary technique. Only $25 \%$ of patients felt that they had been adequately informed about complementary techniques. Up to $80 \%$ expressed interest in the use of complementary therapies in future.

Thirty- $70 \%$ of patients did not inform their treating physician that they were using complementary methods. Physicians who administer conventional therapies underestimate both the use of complementary therapies and the frequency with which this use is not disclosed by the patient. In the studies, reasons given for the use of complementary medicinal techniques include seeking the optimal therapy, the desire to do without steroids, side-effects of conventional therapies, the desire to strengthen personal activity and individual responsibility, the holistic therapeutic approach, dissatisfaction with conventional therapies and (relative) therapy failure. In children with Crohn's disease, the use of CAM correlated to the increase in time absent from school, use of internet and more severe course of disease.

\section{RECOMMENDATION 6.2.4}

Due to the high proportion of patients using complementary techniques, physicians should keep informed about these therapies.

Expert consensus, recommendation, consensus

\section{Background}

One of the reasons for patients using CAM is dissatisfaction with conventional therapies (see above). Many feel their complex multidimensional personality to be inadequately addressed by natural science-orientated conventional medicine. The views of the physician and the patient are often very different, not only in terms of understanding and dealing with the disease, but also as regards self-image and outlook on life in general. Thus, consultation and assistance from CAM therapists is sought from sources outside the standard healthcare services, e. g., alternative practitioners. Not infrequently, patients pursue a two-pronged strategy, seeking additional therapeutic options while being treated with conventional therapies by a consultant practitioner. Whereas the latter is not informed that parallel CAM therapies are being used, the CAM therapist lacks expertise in ulcerative colitis. Fifteen-50\% of patients with IBD fail to inform their treating physician that they are using complementary therapies for fear of derogatory remarks on the part of the physician. On the other hand, less than $20 \%$ of IBD consultants question their patients regarding the use of CAM. In order to minimise risks, the arbitrary parallel use of CAM and conventional therapies by the patient should be avoided and therapies should be closely coordinated. Such coordination, and the avoidance of patient-instigated two-pronged treatment strategies, can only succeed if the primary physician has adequate knowledge of the complementary therapy and can offer competent and objective in-depth advice regarding CAM techniques. Ideally, an integrative approach should be sought, in which conventional and complementary therapies merge into a "best practice" (WHO definition of integrative medicine).

\section{Mind-body techniques}

\section{RECOMMENDATION 6.2.5}

Attention-based techniques for stress reduction can be applied complementarily as a means of improving quality of life. Evidence grade 2, recommendation grade 0 , consensus

\section{Background}

A systematic review of studies on complementary and alternative IBD therapies [753] identified an RCT evaluating lifestyle modification over a one-year follow-up period [754, 755]. In a prospective, randomised controlled trial in patients with remission or mild disease activity using the waiting-list design, 10-week lifestyle modification programmes (activity, nutrition programme, self-help strategies, stress management) improved IBDQ scores by a mean of 20 points in the intervention group (an individual improvement of 16 points is considered clinically significant). The SF-36 mental health subscore showed a significant improvement in comparison to the waiting list control group. In the catamnesis after 12 months, a significant difference was no longer detectable between the groups.

A systematic review of complementary and alternative methods for the treatment of IBD identified two RCTs evaluating the effect of "mindfulness"-based therapy in patients with ulcerative colitis [756, 757]. One of these studies included 66 patients with 
ulcerative colitis or Crohn's disease in remission and compared multi-convergent therapy (mindfulness meditation with aspects of cognitive behavioural therapy) in combination with standard therapy with standard therapy alone [756]. A further study included 55 patients with ulcerative colitis in remission and compared mindfulness-based stress reduction (MBSR) with a "time/attention" control group [757]. Neither disease activity nor psychological variables were found to be significantly different after the interventions. An effect on quality of life was found only in patients with irritable bowel syndrome, while patients who suffered a disease flare during the study period showed reduced levels of CRP and stress.

Evidence of effectiveness is available not only for mindfulness, but also for certain other mind-body techniques. A systematic review of complementary and alternative treatment methods in IBD [753] identified an RCT evaluating the effect of relaxation training [758]. Fifty-six patients were randomised to receive relaxation training or standard treatment. Significant improvements were recorded for levels of pain, anxiety, mood, stress and quality of life in the relaxation group, but not in the standard treatment group. The positive effects on pain are backed up by another study which assessed the effect of a relaxation technique on 40 patients with ulcerative colitis and chronic pain [759]. However, the quality of this study is hard to assess, and therefore the results should be interpreted with caution.

In an RCT, the effects of a programme of breathing, movement and meditation (Breath-Body-Mind Workshop, BBMW) on physiological and psychological symptoms in 29 patients with IBD were evaluated and compared with an education group [760]. The study concluded that BBMW can be carried out, since it leads to positive pre- and post-effects. However, an educational seminar achieves similar results. Furthermore, the study methods are questionable and only 9 patients with ulcerative colitis were included. Again, therefore, the results should be interpreted with caution.

Physical activity could have a positive effect on ulcerative colitis, but evidence is sparse. In a prospective RCT, the effect of activity on IBD was evaluated. The 30 participating patients were either in remission or had mild disease activity [761]. After moderate jogging three times a week for ten weeks, there was a significant difference between the groups in terms of social wellbeing. No other inter-group differences were found. This result should be interpreted with caution, since it was a pilot study which did not differentiate between patients with ulcerative colitis and patients with Crohn's disease, had a small patient collective and employed questionable methods. There were no undesired side-effects. A systematic review concludes that physical activity has a positive effect, but importantly, the analysis includes no RCTs [762].

\section{RECOMMENDATION 6.2.6}

Yoga can be used as a complementary technique to improve quality of life.

Evidence grade 2, recommendation grade 0 , consensus

\section{Background}

A randomised, controlled pilot study evaluated the effect of yoga in comparison to standard therapy in 60 patients with ulcerative colitis in remission [763]. At follow-up after 2 months, a significant difference was found with regard to anxiety and colicky abdominal pain in favour of the yoga group. A further randomised, controlled study included 77 patients with ulcerative colitis in remission and a diminished quality of life. Yoga was found to have a positive effect on quality of life at weeks 12 and 24, and additionally on disease activity at week 24 [764].

\section{Holistic therapies}

\section{RECOMMENDATION 6.2.7}

Acupuncture can be applied as a complementary therapy in patients with mild to moderate disease flares.

Evidence grade 3, recommendation grade 0 , consensus

\section{Background}

Two systematic reviews of CAM techniques and acupuncture in the treatment of patients with IBD identified an RCT which had compared acupuncture and moxibustion (10 treatment sessions in 5 weeks) to superficial needling at non-acupuncture points in patients with mild to moderately active ulcerative colitis [753, 765]. The CAI fell significantly as a result of acupuncture therapy in comparison to the control group. For the secondary endpoints, improvement in quality of life and improvement in general condition, no intergroup difference was found at therapy completion [766].

Two meta-analyses which included studies from China concluded that the methodological quality of the available studies is not good enough to allow conclusions to be drawn [767, 768].

\section{Herbal therapies}

\section{RECOMMENDATION 6.2.8}

Plantago ovata can be used as a complementary approach in maintenance therapy.

Evidence grade 2, recommendation grade 0 , consensus

\section{Background}

One hundred and two patients with ulcerative colitis in remission were allocated into 3 groups and received either plantago ovata, plantago ovata in combination with mesalazine, or mesalazine alone. After 12 months, no difference was found between the groups, except that stool concentrations of butyrate were better in the stool of patients who had received plantago ovata. There were no serious adverse events [769]. In Germany, medications containing plantago ovata have been approved. 


\section{STATEMENT 6.2.9}

Studies have shown positive results for the application of curcumin complementary to mesalazine in both the induction and maintenance of remission. Curcumin is not available as a medication in Germany.

Evidence grade 2, recommendation grade 0, majority approval

\section{Background}

A systematic review of CAM therapies in the treatment of IBD identified two RCTs evaluating the effects of curcumin in patients with ulcerative colitis. In a prospective, randomised, double-blind, placebo-controlled multicentre study, $2 \times 1 \mathrm{~g} / \mathrm{d}$ curcumin complementary to sulphasalazine or mesalazine was evaluated for remission maintenance in ulcerative colitis over a period of 6 months (22 patients in the verum group) [770]. After therapy, a significant intergroup difference was observed with regard to relapse frequency, CAl and endoscopic index in favour of the verum group. These positive findings are supported by those of two further high-quality RCTs [771, 772]: curcumin was found to be the superior therapy in ulcerative colitis. In one of the studies, however, these effects were seen only in the per-protocol and not in the intention-to-treat analysis [771]. No difference was detected concerning side-effects. In Germany, curcumin is sold only as a nutritional supplement and is not available as a medication.

\section{RECOMMENDATION 6.2.10}

A combination of myrrh, camomile blossom extract and coffeae carbo can be given as complementary treatment in maintenance therapy.

Evidence grade 2, Recommendation grade 0, Consensus

\section{Background}

A systematic review of CAM therapies for IBD identified a highquality clinical study examining the effectiveness and safety of myrrh, camomile blossom extract and coffeae carbo (Myrrhinil intest $\left.{ }^{\circledR}\right)$ in remission maintenance therapy in a collective of 96 patients with UC [773]. Results of the study suggested the myrrh, camomile blossom extract and coffeae carbo therapy to be very well tolerated and no less effective than a standard mesalazine therapy in remission maintenance therapy. A follow-up questionnaire-based survey and a large cohort study corroborated these findings [774, 775].

In Germany, there is an approved traditional medication containing the combination of myrrh, camomile blossom extract and coffeae carbo.

\section{RECOMMENDATION 6.2.11}

Other unconventional remedies cannot be recommended due to insufficient data.

Expert consensus, recommendation open, consensus

\section{Background}

The effect of pomegranate (punica granatum peel) extract in combination with standard therapy in comparison to placebo with standard therapy was evaluated in 78 patients with moderately active ulcerative colitis. The study concluded that pomegranate extract can be administered, as it had positive effects on disease activity and certain symptoms. However, these effects were not superior to placebo. Side-effects were mild to moderate and did not differ between the groups [776].

A systematic review [753] identified two non-randomised studies which compared the effectiveness of Boswellia serrata (Indian frankincense) with that of sulphasalazine. Positive effects with respect to histology and stool parameters were detected in both groups, without significant intergroup differences. Sideeffects included indigestion, nausea, loss of appetite and upper abdominal pain [777, 778]. Boswellia serrata is marketed in Germany only as a nutrition supplement and is not available as a medication.

A systematic review [753] identified two RCTs of high quality that evaluated the effects of HMPL-004, the main ingredient of which is Andrographis paniculata (an Ayurveda plant also known as kalmegh or kalmegha) in a total of 344 patients with ulcerative colitis. In one of these studies, the patients were treated for eight weeks with either HMPL-004 or mesalazine [779]. While both preparations had a positive effect on disease activity, there were more side-effects in the mesalazine group. In the second study, two doses of HMPL-004 in combination with mesalazine $(1200 \mathrm{mg}$ and $1800 \mathrm{mg}$ ) or placebo in combination with mesalazine were given for a period of eight weeks and compared with one another [780]. After eight weeks, the higher dose of HMPL004 was found to be superior to placebo in terms of clinical response to therapy and mucosal healing, but not in terms of clinical remission. HMPL-004 is not available as a medication in Germany.

An RCT testing the effect of wheat grass juice compared to placebo juice in 24 patients with ulcerative colitis showed that after four weeks, the patients in the experimental group had a significant reduction in disease activity, rectal bleeding and abdominal pain [781]. No serious side-effects occurred.

A systematic review of CAM therapies in the treatment of inflammatory bowel disease [753] identified an RCT that studied the effect of evening primrose oil in 43 patients with ulcerative colitis in comparison to olive oil and omega 3 oil [782]. Evening primrose showed superiority only in terms of stool consistency. Adverse effects were not documented.

In a prospective, randomised, double blind, placebo-controlled study, $100 \mathrm{ml}$ aloe vera gel was tested over a period of 4 weeks (30 patients with verum: 14 patients with placebo) in patients with mild to moderate ulcerative colitis [783]. Due to statistical flaws, a therapy recommendation cannot be given on the basis of the study's results.

An RCT evaluated the effect of silymarin (milk thistle extract) compared with placebo in 80 patients [784]. No significant intergroup differences were observed.

A further RCT compared the effectiveness of sophora (extract of the Japanese pagoda tree in capsule form) to that of mesalazine in 126 patients with UC [785]. The findings suggest that the treat- 
ment with sophora may not be inferior to the standard therapy with mesalazine. There were no relevant side-effects.

An open pilot study showed that an oral bilberry preparation consisting of dried fruits and juice concentrate may have a positive effect on disease activity [786]. However, well-designed RCTs are necessary in order to verify these results.

Two randomised, placebo-controlled clinical studies including 141 patients with ulcerative colitis show that transdermal nicotine has additional positive effects when combined with standard therapy in patients with ulcerative colitis $[787,788]$. On the other hand, it appears to be ineffective in remission maintenance and as a monotherapy in active colitis [789, 790]. However, the administration of transdermal nicotine, especially to lifelong non-smokers, frequently causes side-effects, some of which are severe.

A randomised, controlled pilot study compared an oxygen therapy plus standard medication to standard therapy alone in patients with active ulcerative colitis [791]. While the oxygen therapy showed no significant effects, significance could not have been shown in any case due to the inadequate sample size.

A review included an RCT studying trichuris-suis eggs in ulcerative colitis [792]. In this prospective, randomised, double-blind, placebo-controlled monocentric trial, a therapy with 2500 trichuris-suis eggs every 2 weeks over 12 weeks was evaluated in patients with active ulcerative colitis (CAI >4) [793]. At the end of the therapy, there was a significant intergroup difference in CAI scores in favour of the verum group. No serious side-effects were evident. The preparation is not approved for medicinal purposes in Germany. Since a large, randomised-controlled study of its efficacy for remission induction in Crohn's disease failed to show a positive effect [794], this therapeutic approach is currently no longer being pursued. It is not possible to issue a recommendation for therapy with trichuris-suis ovata (TSO).

$\begin{array}{ll}\text { ABBREVIATIONS } \\ \text { 5-ASA } & \text { 5-Aminosalicylates } \\ \text { ACT } & \begin{array}{l}\text { Austrian Cholesterol Screening and Treatment } \\ \text { Program }\end{array} \\ \text { ALGK } & \begin{array}{l}\text { Arbeitsgemeinschaft gastroenterologischer } \\ \text { Klinikärzte e. V.. }\end{array} \\ \text { ALM } & \text { Adenoma-associated lesion or mass } \\ \text { ASCA } & \text { Anti-saccharomyces cerevisiae antibodies } \\ \text { AWMF } & \text { Arbeitsgemeinschaft der Wissenschaftlichen } \\ & \text { Medizinischen Fachgesellschaften e. V. } \\ \text { AZA } & \text { Azathioprine } \\ \text { BMI } & \text { Body mass index } \\ \text { BW } & \text { Body weight } \\ \text { C. difficile } & \text { Clostridium difficile } \\ \text { CAM } & \text { Complementary and alternative medicine } \\ \text { CC } & \text { Consensus conference } \\ \text { CDI } & \text { Clostridium difficile infection } \\ \text { CEBM } & \text { Centre for Evidence-Based Medicine } \\ \text { CEUS } & \text { Contrast-enhanced ultrasound } \\ \text { CGS } & \text { Clinical guideline services } \\ \text { CMV } & \text { Cytomegalovirus } \\ \text { CRP } & \text { C-reactive protein, inflammatory marker } \\ \text { CSA } & \text { Ciclosporin A }\end{array}$

\begin{tabular}{|c|c|}
\hline CT & Computed tomography \\
\hline DALM & Dysplasia-associated lesion or mass \\
\hline DCCV & $\begin{array}{l}\text { Deutsche Morbus Crohn/Colitis ulcerosa } \\
\text { Vereinigung }\end{array}$ \\
\hline DEGAM & $\begin{array}{l}\text { Deutsche Gesellschaft für Allgemeinmedizin } \\
\text { und Familienmedizin }\end{array}$ \\
\hline DEGIM & Deutsche Gesellschaft für Innere Medizin \\
\hline DELBI & $\begin{array}{l}\text { Deutsches Instrument zur methodischen } \\
\text { Leitlinien-Bewertung }\end{array}$ \\
\hline DGAV & $\begin{array}{l}\text { Deutsche Gesellschaft für Allgemein- und } \\
\text { Viszeralchirurgie }\end{array}$ \\
\hline DGCH & Deutsche Gesellschaft für Chirurgie \\
\hline DGEM & Deutsche Gesellschaft für Ernährungsmedizin \\
\hline DGK & Deutsche Gesellschaft für Koloproktologie \\
\hline DGP & Deutsche Gesellschaft für Pathologie \\
\hline DGVS & $\begin{array}{l}\text { Deutsche Gesellschaft für Gastroenterologie, } \\
\text { Verdauungs- und Stoffwechselkrankheiten }\end{array}$ \\
\hline DPGE & $\begin{array}{l}\text { Gesellschaft für pädiatrische Gastroenterologie } \\
\text { und Ernährungsmedizin }\end{array}$ \\
\hline E. coli & Escherichia coli \\
\hline EBM & Evidence-based medicine \\
\hline EBV & Epstein Barr virus \\
\hline ECCO & European Crohn's and Colitis Organization \\
\hline EcN & Escherichia coli Nissle \\
\hline EMA & European Medicines Agency \\
\hline ESCMID & $\begin{array}{l}\text { European Society of Clinical Microbiology and } \\
\text { Infectious Diseases }\end{array}$ \\
\hline ESPEN & $\begin{array}{l}\text { European Society for Parenteral and Enteral } \\
\text { Nutrition }\end{array}$ \\
\hline ESPGHAN & $\begin{array}{l}\text { European Society of Paediatric Gastroenterology, } \\
\text { Hepatology and Nutrition }\end{array}$ \\
\hline ESR & Erythrocyte sedimentation rate \\
\hline FACED & $\begin{array}{l}\text { Fachangestellte für chronisch entzündliche } \\
\text { Darmerkrankungen }\end{array}$ \\
\hline FICE & Flexible intelligent colour enhancement \\
\hline FMT & Faecal microbiota transplantation \\
\hline GDH & Glutamate dehydrogenase \\
\hline $\mathrm{Hb}$ & Haemoglobin \\
\hline HBV & Hepatitis B virus \\
\hline HDWLE & High definition white light endoscopy \\
\hline HGIEN & High grade intraepithelial neoplasia \\
\hline HIV & Human immunodeficiency virus \\
\hline $\mathrm{HLH}$ & Haemophagocytic Lymphohistiocytosis \\
\hline i. m. & Intramuscular \\
\hline IAP & Ileoanal pouch \\
\hline IBD & Inflammatory bowel disease \\
\hline IBDQ & Inflammatory Bowel Disease Questionnaire \\
\hline IBS & Irritable bowel syndrome \\
\hline IEN & Intraepithelial neoplasia \\
\hline IfSG & Infektionsschutzgesetz \\
\hline IFX & Infliximab \\
\hline IGRA & Interferon gamma release assay \\
\hline INH & Isonicotinic acid hydrazide \\
\hline IPAA & Ileoanal pouch-anal anastomosis \\
\hline IPS & Irritable pouch syndrome \\
\hline KRK & Colorectal carcinoma \\
\hline
\end{tabular}




\begin{tabular}{|c|c|}
\hline LBTI & Latent tuberculosis infection \\
\hline LGIEN & Low-grade intraepithelial neoplasia \\
\hline MAS & Macrophage activation syndrome \\
\hline MBSR & Mindfulness-Based Stress Reduction \\
\hline MMA & Methylmalonic acid \\
\hline MMX & Multi matrix system \\
\hline MRI & Magnetic resonance imaging \\
\hline MR & Magnetic resonance \\
\hline MTX & Methotrexate \\
\hline $\mathrm{n}$ & Number \\
\hline $\mathrm{NBI}$ & Narrow band imaging \\
\hline NRS & Non-randomised studies \\
\hline NSAR & Non-steroidal antirheumatic drug \\
\hline PANCA & $\begin{array}{l}\text { Perinuclear anti-neutrophil cytoplasmic } \\
\text { antibodies }\end{array}$ \\
\hline PCR & Polymerase chain reaction \\
\hline PDAI & Perianal Disease Activity Index \\
\hline PEG & Percutaneous endoscopic gastrostomy \\
\hline PJP & Pneumocystis jirovecii pneumoniae \\
\hline PMN & Polymorphonuclear \\
\hline PSC & Primary sclerosing cholangitis \\
\hline PTLD & Post-transplantation lymphoproliferative disease \\
\hline PUCAI & Paediatric Ulcerative Colitis Activity Index \\
\hline RLD & Raised lesions with dysplasia \\
\hline s. C. & Subcutaneous \\
\hline SMX & Sulphamethoxazole \\
\hline TB & Tuberculosis \\
\hline TMP/SMX & $\begin{array}{l}\text { Trimethoprim/sulphamethoxazole } \\
\text { (co-trimoxazole) }\end{array}$ \\
\hline TNF & Tumour necrosis factor \\
\hline TPN & Total parenteral nutrition \\
\hline TSO & Trichuris-suis ovata \\
\hline TST & Tuberculin skin test \\
\hline UC & Ulcerative colitis \\
\hline UCEIS & Ulcerative Colitis Endoscopic Index of Severity \\
\hline VZV & Varicella zoster virus \\
\hline WHO & World Health Organisation \\
\hline WLE & White light endoscopy \\
\hline
\end{tabular}

\section{Acknowledgements}

We are particularly grateful to Janet Collins, Interdisciplinary Crohn Colitis Centre Rhein-Main, Frankfurt am Main, Germany for the translation of the German manuscript and to PD Dr. Petra Lynen-Jansen, DGVS, Berlin, for substantial contribution to coordinating the Consensus.

\section{References}

[1] Marteau P, Probert CS, Lindgren S et al. Combined oral and enema treatment with Pentasa (mesalazine) is superior to oral therapy alone in patients with extensive mild/moderate active ulcerative colitis: a randomised, double blind, placebo controlled study. Gut 2005; 54: 960 - 965

[2] Silverberg MS, Satsangi J, Ahmad T et al. Toward an integrated clinical, molecular and serological classification of inflammatory bowel disease: Report of a Working Party of the 2005 Montreal World Congress of Gastroenterology. Can J Gastroenterol 2005; 19: 5-36
[3] Ekbom A, Helmick C, Zack M et al. Ulcerative colitis and colorectal cancer. A population-based study. N Engl J Med 1990; 323: 1228-1233

[4] Soetikno RM, Lin OS, Heidenreich PA et al. Increased risk of colorectal neoplasia in patients with primary sclerosing cholangitis and ulcerative colitis: a meta-analysis. Gastrointest Endosc 2002; 56: 48 - 54

[5] Forrest K, Symmons D, Foster P. Systematic review: is ingestion of paracetamol or non-steroidal anti-inflammatory drugs associated with exacerbations of inflammatory bowel disease? Aliment Pharmacol Ther 2004; 20: 1035 - 1043

[6] Takeuchi K, Smale S, Premchand P et al. Prevalence and mechanism of nonsteroidal anti-inflammatory drug-induced clinical relapse in patients with inflammatory bowel disease. Clin Gastroenterol Hepatol 2006; 4: $196-202$

[7] Sandborn WJ, Stenson WF, Brynskov J et al. Safety of celecoxib in patients with ulcerative colitis in remission: a randomized, placebocontrolled, pilot study. Clin Gastroenterol Hepatol 2006; 4: 203 -211

[8] Korzenik JR, Podolsky DK. Selective use of selective nonsteroidal antiinflammatory drugs in inflammatory bowel disease. Clin Gastroenterol Hepatol 2006; 4: 157 - 159

[9] Ho GT, Chiam P, Drummond $\mathrm{H}$ et al. The efficacy of corticosteroid therapy in inflammatory bowel disease: analysis of a 5-year UK inception cohort. Aliment Pharmacol Ther 2006; 24: 319-330

[10] Faubion WA Jr, Loftus EV Jr, Harmsen WS et al. The natural history of corticosteroid therapy for inflammatory bowel disease: a populationbased study. Gastroenterology 2001; 121: 255-260

[11] Toruner M, Loftus EV Jr, Harmsen WS et al. Risk factors for opportunistic infections in patients with inflammatory bowel disease. Gastroenterology 2008; 134: 929-936

[12] Mahid SS, Minor KS, Soto RE et al. Smoking and inflammatory bowel disease: a meta-analysis. Mayo Clin Proc 2006; 81: 1462 - 1471

[13] Hoie O, Wolters F, Riis L et al. Ulcerative colitis: patient characteristics may predict 10-yr disease recurrence in a European-wide populationbased cohort. Am J Gastroenterol 2007; 102: 1692-1701

[14] Beaugerie L, Massot N, Carbonnel F et al. Impact of cessation of smoking on the course of ulcerative colitis. Am J Gastroenterol 2001; 96: 2113 2116

[15] Birrenbach T, Bocker U. Inflammatory bowel disease and smoking: a review of epidemiology, pathophysiology, and therapeutic implications. Inflamm Bowel Dis 2004; 10: 848-859

[16] Florin TH, Pandeya N, Radford-Smith GL. Epidemiology of appendicectomy in primary sclerosing cholangitis and ulcerative colitis: its influence on the clinical behaviour of these diseases. Gut 2004; 53: $973-979$

[17] Vermeire S. Review article: genetic susceptibility and application of genetic testing in clinical management of inflammatory bowel disease. Aliment Pharmacol Ther 2006; 24 (Suppl. 3): 2-10

[18] Merrett MN, Mortensen N, Kettlewell M et al. Smoking may prevent pouchitis in patients with restorative proctocolectomy for ulcerative colitis. Gut 1996; 38: $362-364$

[19] Joelsson M, Benoni C, Oresland T. Does smoking influence the risk of pouchitis following ileal pouch anal anastomosis for ulcerative colitis? Scand J Gastroenterol 2006; 41: 929-933

[20] Loftus EV Jr. Clinical epidemiology of inflammatory bowel disease: Incidence, prevalence, and environmental influences. Gastroenterology 2004; 126: $1504-1517$

[21] Rutgeerts P, D'Haens G, Hiele M et al. Appendectomy protects against ulcerative colitis. Gastroenterology 1994; 106: 1251 - 1253

[22] Frisch M, Johansen C, Mellemkjaer L et al. Appendectomy and subsequent risk of inflammatory bowel diseases. Surgery 2001; 130: 36 - 43

[23] Koutroubakis IE, Vlachonikolis IG, Kouroumalis EA. Role of appendicitis and appendectomy in the pathogenesis of ulcerative colitis: a critical review. Inflamm Bowel Dis 2002; 8: 277 - 286 
[24] Cosnes ], Carbonnel F, Beaugerie L et al. Effects of appendicectomy on the course of ulcerative colitis. Gut 2002; 51: 803-807

[25] Radford-Smith GL, Edwards JE, Purdie DM et al. Protective role of appendicectomy on onset and severity of ulcerative colitis and Crohn's disease. Gut 2002; 51: 808-813

[26] Childers RE, Eluri S, Vazquez C et al. Family history of inflammatory bowel disease among patients with ulcerative colitis: a systematic review and meta-analysis. J Crohns Colitis 2014; 8: 1480-1497

[27] Henriksen M, Jahnsen J, Lygren I et al. Are there any differences in phenotype or disease course between familial and sporadic cases of inflammatory bowel disease? Results of a population-based follow-up study. Am J Gastroenterol 2007; 102: 1955-1963

[28] IBD Working Group of the European Society for Paediatric Gastroenterology, Hepatology and Nutrition. Inflammatory bowel disease in children and adolescents: recommendations for diagnosis-the Porto criteria. J Pediatr Gastroenterol Nutr 2005; 41: 1 - 7

[29] Langholz E, Munkholm P, Davidsen M et al. Course of ulcerative colitis: analysis of changes in disease activity over years. Gastroenterology 1994; 107: 3-11

[30] Henriksen M, Jahnsen J, Lygren I et al. Change of diagnosis during the first five years after onset of inflammatory bowel disease: results of a prospective follow-up study (the IBSEN Study). Scand J Gastroenterol 2006; 41: 1037 - 1043

[31] Price AB. Overlap in the spectrum of non-specific inflammatory bowel disease-'colitis indeterminate'. J Clin Pathol 1978; 31: 567 - 577

[32] Rodgers AD, Cummins AG. CRP correlates with clinical score in ulcerative colitis but not in Crohn's disease. Dig Dis Sci 2007; 52: 2063 - 2068

[33] Vermeire S, Van Assche G, Rutgeerts P. C-reactive protein as a marker for inflammatory bowel disease. Inflamm Bowel Dis 2004; 10: 661 - 665

[34] Prantera C, Davoli M, Lorenzetti R et al. Clinical and laboratory indicators of extent of ulcerative colitis. Serum C-reactive protein helps the most. J Clin Gastroenterol 1988; 10: 41 - 45

[35] Roseth AG, Aadland E, Grzyb K. Normalization of faecal calprotectin: a predictor of mucosal healing in patients with inflammatory bowel disease. Scand J Gastroenterol 2004; 39: 1017-1020

[36] Schoepfer AM, Beglinger C, Straumann A et al. Ulcerative colitis: Correlation of the Rachmilewitz endoscopic activity index with fecal calprotectin, clinical activity, C-reactive protein, and blood leukocytes. Inflamm Bowel Dis 2009; 15: 1851 - 1858

[37] Mao R, Xiao YL, Gao X et al. Fecal calprotectin in predicting relapse of inflammatory bowel diseases: a meta-analysis of prospective studies. Inflamm Bowel Dis 2012; 18: $1894-1899$

[38] Lin JF, Chen JM, Zuo JH et al. Meta-analysis: fecal calprotectin for assessment of inflammatory bowel disease activity. Inflamm Bowel Dis 2014; 20: $1407-1415$

[39] Korczowski B, Szybist W. Serum procalcitonin and C-reactive protein in children with diarrhoea of various aetiologies. Acta Paediatr 2004; 93 : $169-173$

[40] Herrlinger KR, Dittmann R, Weitz G et al. Serum procalcitonin differentiates inflammatory bowel disease and self-limited colitis. Inflamm Bowel Dis 2004; 10: 229-233

[41] Mylonaki M, Langmead L, Pantes A et al. Enteric infection in relapse of inflammatory bowel disease: importance of microbiological examination of stool. Eur J Gastroenterol Hepatol 2004; 16: 775-778

[42] Brown W], Hudson MJ, Patrick S et al. Search for enteric microbial pathogens in patients with ulcerative colitis. Digestion 1992; 53: $121-128$

[43] Bou-Fakhredin R, Halawi R, Roumi J et al. Insights into the diagnosis and management of iron deficiency in inflammatory bowel disease. Expert Rev Hematol 2017; 10: $801-808$

[44] Stein J, Dignass AU. Management of iron deficiency anemia in inflammatory bowel disease - a practical approach. Ann Gastroenterol 2013; 26: $104-113$
[45] Cappellini MD, Comin-Colet J, de Francisco A et al. Iron deficiency across chronic inflammatory conditions: International expert opinion on definition, diagnosis, and management. Am J Hematol 2017; 92: 1068 1078

[46] Stein J, Hartmann F, Dignass AU. Diagnosis and management of iron deficiency anemia in patients with IBD. Nat Rev Gastroenterol Hepatol 2010; 7: 599-610

[47] Plevy S. Do serological markers and cytokines determine the indeterminate? J Clin Gastroenterol 2004; 38: S51 - S56

[48] Riis L, Vind I, Vermeire $S$ et al. The prevalence of genetic and serologic markers in an unselected European population-based cohort of IBD patients. Inflamm Bowel Dis 2007; 13: 24-32

[49] Joossens S, Daperno M, Shums Z et al. Interassay and interobserver variability in the detection of anti-neutrophil cytoplasmic antibodies in patients with ulcerative colitis. Clin Chem 2004; 50: 1422-1425

[50] Reese GE, Constantinides VA, Simillis C et al. Diagnostic precision of antiSaccharomyces cerevisiae antibodies and perinuclear antineutrophil cytoplasmic antibodies in inflammatory bowel disease. Am J Gastroenterol 2006; 101: 2410-2422

[51] Weber P, Koch M, Heizmann WR et al. Microbic superinfection in relapse of inflammatory bowel disease. J Clin Gastroenterol 1992; 14: 302 - 308

[52] Issa M, Vijayapal A, Graham MB et al. Impact of Clostridium difficile on inflammatory bowel disease. Clin Gastroenterol Hepatol 2007; 5 : $345-351$

[53] Rodemann JF, Dubberke ER, Reske KA et al. Incidence of Clostridium difficile infection in inflammatory bowel disease. Clin Gastroenterol Hepatol 2007; 5: $339-344$

[54] Johal SS, Hammond J, Solomon K et al. Clostridium difficile associated diarrhoea in hospitalised patients: onset in the community and hospital and role of flexible sigmoidoscopy. Gut 2004; 53: 673-677

[55] Minami M, Ohta M, Ohkura T et al. Cytomegalovirus infection in severe ulcerative colitis patients undergoing continuous intravenous cyclosporine treatment in Japan. World J Gastroenterol 2007; 13: 754-760

[56] Matsuoka K, Iwao Y, Mori T et al. Cytomegalovirus is frequently reactivated and disappears without antiviral agents in ulcerative colitis patients. Am J Gastroenterol 2007; 102: 331 - 337

[57] Dimitroulia E, Spanakis N, Konstantinidou AE et al. Frequent detection of cytomegalovirus in the intestine of patients with inflammatory bowel disease. Inflamm Bowel Dis 2006; 12: 879-884

[58] Hommes DW, Sterringa G, van Deventer S] et al. The pathogenicity of cytomegalovirus in inflammatory bowel disease: a systematic review and evidence-based recommendations for future research. Inflamm Bowel Dis 2004; 10: $245-250$

[59] Poullis A, Foster R, Northfield TC et al. Review article: faecal markers in the assessment of activity in inflammatory bowel disease. Aliment Pharmacol Ther 2002; 16: 675-681

[60] Langhorst ], Elsenbruch S, Mueller T et al. Comparison of 4 neutrophilderived proteins in feces as indicators of disease activity in ulcerative colitis. Inflamm Bowel Dis 2005; 11: 1085 - 1091

[61] Menees SB, Powell C, Kurlander ] et al. A meta-analysis of the utility of $C$-reactive protein, erythrocyte sedimentation rate, fecal calprotectin, and fecal lactoferrin to exclude inflammatory bowel disease in adults with IBS. Am J Gastroenterol 2015; 110: 444-454

[62] Mosli MH, Zou G, Garg SK et al. C-Reactive Protein, Fecal Calprotectin, and Stool Lactoferrin for Detection of Endoscopic Activity in Symptomatic Inflammatory Bowel Disease Patients: A Systematic Review and Meta-Analysis. Am J Gastroenterol 2015; 110: 802 - 819; quiz 820

[63] D'Inca R, Dal Pont E, Di Leo V et al. Can calprotectin predict relapse risk in inflammatory bowel disease? Am J Gastroenterol 2008; 103: 2007 2014 
[64] Costa F, Mumolo MG, Bellini M et al. Role of faecal calprotectin as noninvasive marker of intestinal inflammation. Dig Liver Dis 2003; 35: 642 647

[65] van Rheenen PF, Van de Vijver E, Fidler V. Faecal calprotectin for screening of patients with suspected inflammatory bowel disease: diagnostic meta-analysis. BMJ 2010; 341: c3369

[66] Van de Vijver E, Schreuder AB, Cnossen WR et al. Safely ruling out inflammatory bowel disease in children and teenagers without referral for endoscopy. Arch Dis Child 2012; 97: 1014-1018

[67] Walker TR, Land ML, Kartashov A et al. Fecal lactoferrin is a sensitive and specific marker of disease activity in children and young adults with inflammatory bowel disease. J Pediatr Gastroenterol Nutr 2007; 44: 414 422

[68] Joishy M, Davies I, Ahmed M et al. Fecal calprotectin and lactoferrin as noninvasive markers of pediatric inflammatory bowel disease. J Pediatr Gastroenterol Nutr 2009; 48: 48 - 54

[69] Costa F, Mumolo MG, Ceccarelli L et al. Calprotectin is a stronger predictive marker of relapse in ulcerative colitis than in Crohn's disease. Gut 2005; 54: $364-368$

[70] Walkiewicz D, Werlin SL, Fish D et al. Fecal calprotectin is useful in predicting disease relapse in pediatric inflammatory bowel disease. Inflamm Bowel Dis 2008; 14: 669-673

[71] Carbonnel F, Lavergne A, Lemann M et al. Colonoscopy of acute colitis. A safe and reliable tool for assessment of severity. Dig Dis Sci 1994; 39 $1550-1557$

[72] Alemayehu G, Jarnerot G. Colonoscopy during an attack of severe ulcerative colitis is a safe procedure and of great value in clinical decision making. Am J Gastroenterol 1991; 86: 187-190

[73] Fefferman DS, Farrell RJ. Endoscopy in inflammatory bowel disease: indications, surveillance, and use in clinical practice. Clin Gastroenterol Hepatol 2005; 3: $11-24$

[74] Deutsch DE, Olson AD. Colonoscopy or sigmoidoscopy as the initial evaluation of pediatric patients with colitis: a survey of physician behavior and a cost analysis. J Pediatr Gastroenterol Nutr 1997; 25: 26 - 31

[75] Melmed GY, Elashoff R, Chen GC et al. Predicting a change in diagnosis from ulcerative colitis to Crohn's disease: a nested, case-control study. Clin Gastroenterol Hepatol 2007; 5: 602-608; quiz 525

[76] Bryant RV, Burger DC, Delo J et al. Beyond endoscopic mucosal healing in UC: histological remission better predicts corticosteroid use and hospitalisation over 6 years of follow-up. Gut 2016; 65: 408 -414

[77] Neurath MF, Travis SP. Mucosal healing in inflammatory bowel diseases: a systematic review. Gut 2012; 61: 1619-1635

[78] Shah SC, Colombel JF, Sands BE et al. Mucosal Healing Is Associated With Improved Long-term Outcomes of Patients With Ulcerative Colitis: A Systematic Review and Meta-analysis. Clin Gastroenterol Hepatol 2016; 14: $1245-1255$ e8

[79] Reinink AR, Lee TC, Higgins PD. Endoscopic Mucosal Healing Predicts Favorable Clinical Outcomes in Inflammatory Bowel Disease: A Meta-analysis. Inflamm Bowel Dis 2016; 22: 1859-1869

[80] Gisbert JP, Marin AC, Chaparro M. The Risk of Relapse after Anti-TNF Discontinuation in Inflammatory Bowel Disease: Systematic Review and Meta-Analysis. Am J Gastroenterol 2016; 111: 632 -647

[81] Schroeder KW, Tremaine WJ, Ilstrup DM. Coated oral 5-aminosalicylic acid therapy for mildly to moderately active ulcerative colitis. A randomized study. N Engl J Med 1987; 317: 1625 - 1629

[82] Travis SP, Schnell D, Krzeski P et al. Developing an instrument to assess the endoscopic severity of ulcerative colitis: the Ulcerative Colitis Endoscopic Index of Severity (UCEIS). Gut 2012; 61: 535 - 542

[83] Truelove SC, Witts LJ. Cortisone in ulcerative colitis; final report on a therapeutic trial. Br Med J 1955; 2: 1041 - 1048

[84] Jakobovits SL, Travis SP. Management of acute severe colitis. Br Med Bull 2005; 75-76: 131 - 144
[85] Turner D, Otley AR, Mack D et al. Development, validation, and evaluation of a pediatric ulcerative colitis activity index: a prospective multicenter study. Gastroenterology 2007; 133: 423-432

[86] Preiss JC, Bokemeyer B, Buhr HJ et al. Updated German clinical practice guideline on "Diagnosis and treatment of Crohn's disease" 2014. Z Gastroenterol 2014; 52: 1431 - 1484

[87] Parente F, Greco S, Molteni M et al. Role of early ultrasound in detecting inflammatory intestinal disorders and identifying their anatomical location within the bowel. Aliment Pharmacol Ther 2003; 18: 1009 1016

[88] Hollerbach S, Geissler A, Schiegl $\mathrm{H}$ et al. The accuracy of abdominal ultrasound in the assessment of bowel disorders. Scand J Gastroenterol 1998; 33: $1201-1208$

[89] Maconi G, Ardizzone S, Parente F et al. Ultrasonography in the evaluation of extension, activity, and follow-up of ulcerative colitis. Scand J Gastroenterol 1999; 34: 1103-1107

[90] Worlicek H, Lutz H, Heyder N et al. Ultrasound findings in Crohn's disease and ulcerative colitis: a prospective study. J Clin Ultrasound 1987; 15: $153-163$

[91] Bozkurt T, Richter F, Lux G. Ultrasonography as a primary diagnostic tool in patients with inflammatory disease and tumors of the small intestine and large bowel. J Clin Ultrasound 1994; 22: 85-91

[92] Arienti V, Campieri M, Boriani L et al. Management of severe ulcerative colitis with the help of high resolution ultrasonography. Am J Gastroenterol 1996; 91: 2163-2169

[93] Faure C, Belarbi N, Mougenot JF et al. Ultrasonographic assessment of inflammatory bowel disease in children: comparison with ileocolonoscopy. J Pediatr 1997; 130: 147-151

[94] Pradel JA, David XR, Taourel P et al. Sonographic assessment of the normal and abnormal bowel wall in nondiverticular ileitis and colitis. Abdom Imaging 1997; 22: $167-172$

[95] Haber HP, Busch A, Ziebach R et al. Ultrasonographic findings correspond to clinical, endoscopic, and histologic findings in inflammatory bowel disease and other enterocolitides. J Ultrasound Med 2002; 21: $375-382$

[96] Pascu M, Roznowski AB, Muller HP et al. Clinical relevance of transabdominal ultrasonography and magnetic resonance imaging in patients with inflammatory bowel disease of the terminal ileum and large bowel. Inflamm Bowel Dis 2004; 10: 373 - 382

[97] Parente F, Molteni M, Marino B et al. Are Colonoscopy and Bowel Ultrasound Useful for Assessing Response to Short-Term Therapy and Predicting Disease Outcome of Moderate-to-Severe Forms of Ulcerative Colitis?: A Prospective Study. Am J Gastroenterol 2010; 105: $1150-1157$

[98] Ludwig D, Wiener S, Bruning A et al. Mesenteric blood flow is related to disease activity and risk of relapse in ulcerative colitis: a prospective follow up study. Gut 1999; 45: 546-552

[99] Homann N, Klarmann U, Fellermann K et al. Mesenteric pulsatility index analysis predicts response to azathioprine in patients with Crohn's disease. Inflamm Bowel Dis 2005; 11: 126 -132

[100] Girlich C, Schacherer D, Jung EM et al. Comparison between quantitative assessment of bowel wall vascularization by contrast-enhanced ultrasound and results of histopathological scoring in ulcerative colitis. Int | Colorectal Dis 2012; 27: $193-198$

[101] Rutter MD, Saunders BP, Wilkinson KH et al. Cancer surveillance in longstanding ulcerative colitis: endoscopic appearances help predict cancer risk. Gut 2004; 53: 1813-1816

[102] Gumaste V, Sachar DB, Greenstein AJ. Benign and malignant colorectal strictures in ulcerative colitis. Gut 1992; 33: 938 - 941

[103] Reiser JR, Waye JD, Janowitz HD et al. Adenocarcinoma in strictures of ulcerative colitis without antecedent dysplasia by colonoscopy. Am J Gastroenterol 1994; 89: 119-122 
[104] Andersen K, Vogt C, Blondin D et al. Multi-detector CT-colonography in inflammatory bowel disease: prospective analysis of CT-findings to high-resolution video colonoscopy. Eur J Radiol 2006; 58: 140-146

[105] Horsthuis K, Bipat S, Bennink RJ et al. Inflammatory bowel disease diagnosed with US, MR, scintigraphy, and CT: meta-analysis of prospective studies. Radiology 2008; 247: 64-79

[106] Fumery M, Pineton de Chambrun G, Stefanescu C et al. Detection of Dysplasia or Cancer in 3.5\% of Patients With Inflammatory Bowel Disease and Colonic Strictures. Clin Gastroenterol Hepatol 2015; 13: $1770-1775$

[107] Sawczenko A, Sandhu BK. Presenting features of inflammatory bowel disease in Great Britain and Ireland. Arch Dis Child 2003; 88: 995 1000

[108] Ajaj WM, Lauenstein TC, Pelster G et al. Magnetic resonance colonography for the detection of inflammatory diseases of the large bowel: quantifying the inflammatory activity. Gut 2005; 54: 257-263

[109] Levine A, Koletzko S, Turner D et al. ESPGHAN revised porto criteria for the diagnosis of inflammatory bowel disease in children and adolescents. J Pediatr Gastroenterol Nutr 2014; 58: 795-806

[110] Allison MC, Hamilton-Dutoit S], Dhillon AP et al. The value of rectal biopsy in distinguishing self-limited colitis from early inflammatory bowel disease. Q J Med 1987; 65: 985 - 995

[111] Bentley E, Jenkins D, Campbell F et al. How could pathologists improve the initial diagnosis of colitis? Evidence from an international workshop. J Clin Pathol 2002; 55: 955 - 960

[112] Dejaco C, Oesterreicher C, Angelberger S et al. Diagnosing colitis: a prospective study on essential parameters for reaching a diagnosis. Endoscopy 2003; 35: 1004 - 1008

[113] Dube AK, Cross SS, Lobo AJ. Audit of the histopathological diagnosis of non-neoplastic colorectal biopsies: achievable standards for the diagnosis of inflammatory bowel disease. J Clin Pathol 1998; 51: 378-381

[114] Dundas SA, Dutton J, Skipworth P. Reliability of rectal biopsy in distinguishing between chronic inflammatory bowel disease and acute selflimiting colitis. Histopathology 1997; 31: 60-66

[115] Jenkins D, Balsitis M, Gallivan S et al. Guidelines for the initial biopsy diagnosis of suspected chronic idiopathic inflammatory bowel disease. The British Society of Gastroenterology Initiative. J Clin Pathol 1997; 50: $93-105$

[116] Nostrant TT, Kumar NB, Appelman HD. Histopathology differentiates acute self-limited colitis from ulcerative colitis. Gastroenterology 1987; $92: 318-328$

[117] Schumacher G, Kollberg B, Sandstedt B. A prospective study of first attacks of inflammatory bowel disease and infectious colitis. Histologic course during the 1st year after presentation. Scand J Gastroenterol 1994; 29: $318-332$

[118] Seldenrijk CA, Morson BC, Meuwissen SG et al. Histopathological evaluation of colonic mucosal biopsy specimens in chronic inflammatory bowel disease: diagnostic implications. Gut 1991; 32: 1514-1520

[119] Surawicz CM, Belic L. Rectal biopsy helps to distinguish acute self-limited colitis from idiopathic inflammatory bowel disease. Gastroenterology 1984; 86: $104-113$

[120] Tanaka M, Masuda T, Yao T et al. Observer variation of diagnoses based on simple biopsy criteria differentiating among Crohn's disease, ulcerative colitis, and other forms of colitis. J Gastroenterol Hepatol 2001; 16: $1368-1372$

[121] Tanaka M, Riddell RH, Saito $\mathrm{H}$ et al. Morphologic criteria applicable to biopsy specimens for effective distinction of inflammatory bowel disease from other forms of colitis and of Crohn's disease from ulcerative colitis. Scand J Gastroenterol 1999; 34: 55-67

[122] Tanaka M, Saito H, Fukuda S et al. Simple mucosal biopsy criteria differentiating among Crohn disease, ulcerative colitis, and other forms of colitis: measurement of validity. Scand J Gastroenterol 2000; 35: $281-286$
[123] Theodossi A, Spiegelhalter D], Jass J et al. Observer variation and dis criminatory value of biopsy features in inflammatory bowel disease. Gut 1994; 35: $961-968$

[124] McHugh JB, Appelman HD, McKenna BJ. The diagnostic value of endoscopic terminal ileum biopsies. Am J Gastroenterol 2007; 102: $1084-1089$

[125] Cherian S, Singh P. Is routine ileoscopy useful? An observational study of procedure times, diagnostic yield, and learning curve. Am J Gastroenterol 2004; 99: 2324-2329

[126] Geboes K, Ectors N, D’Haens G et al. Is ileoscopy with biopsy worthwhile in patients presenting with symptoms of inflammatory bowel disease? Am J Gastroenterol 1998; 93: 201 -206

[127] Lemberg DA, Clarkson CM, Bohane TD et al. Role of esophagogastroduodenoscopy in the initial assessment of children with inflammatory bowel disease. J Gastroenterol Hepatol 2005; 20: 1696-1700

[128] Surawicz CM. Serial sectioning of a portion of a rectal biopsy detects more focal abnormalities: a prospective study of patients with inflammatory bowel disease. Dig Dis Sci 1982; 27: 434-436

[129] Surawicz CM, Meisel JL, Ylvisaker T et al. Rectal biopsy in the diagnosis of Crohn's disease: value of multiple biopsies and serial sectioning. Gastroenterology 1981; 80: 66-71

[130] Therkildsen MH, Jensen BN, Teglbjaerg PS et al. The final outcome of patients presenting with their first episode of acute diarrhoea and an inflamed rectal mucosa with preserved crypt architecture. A clinicopathologic study. Scand J Gastroenterol 1989; 24: 158-164

[131] Washington K, Greenson JK, Montgomery E et al. Histopathology of ulcerative colitis in initial rectal biopsy in children. Am J Surg Pathol 2002; 26: 1441 - 1449

[132] Geboes K, Dalle I. Influence of treatment on morphological features of mucosal inflammation. Gut 2002; 50 (Suppl. 3): III37- III42

[133] Odze R, Antonioli D, Peppercorn M et al. Effect of topical 5-aminosalicylic acid (5-ASA) therapy on rectal mucosal biopsy morphology in chronic ulcerative colitis. Am J Surg Pathol 1993; 17: 869-875

[134] Kleer CG, Appelman HD. Ulcerative colitis: patterns of involvement in colorectal biopsies and changes with time. Am J Surg Pathol 1998; 22 983-989

[135] North American Society for Pediatric Gastroenterology H, Nutrition, Colitis Foundation of $A$, et al. Differentiating ulcerative colitis from Crohn disease in children and young adults: report of a working group of the North American Society for Pediatric Gastroenterology, Hepatology, and Nutrition and the Crohn's and Colitis Foundation of America. J Pediatr Gastroenterol Nutr 2007; 44: 653-674

[136] Glickman JN, Bousvaros A, Farraye FA et al. Pediatric patients with untreated ulcerative colitis may present initially with unusual morphologic findings. Am J Surg Pathol 2004; 28: 190-197

[137] Robert ME, Tang L, Hao LM et al. Patterns of inflammation in mucosal biopsies of ulcerative colitis: perceived differences in pediatric populations are limited to children younger than 10 years. Am J Surg Pathol 2004; 28: $183-189$

[138] Markowitz J, Kahn E, Grancher K et al. Atypical rectosigmoid histology in children with newly diagnosed ulcerative colitis. Am J Gastroenterol 1993; 88: 2034-2037

[139] Autschbach F, Hoffmann JCKA, Klump B eds Chronisch entzündliche Darmerkrankungen Das CED-Handbuch für Klinik und Praxis. Stuttgart: Thieme; 2004: 110-122

[140] Borchard F. Differential diagnosis of colitis. Verh Dtsch Ges Pathol 1999; 83: $110-121$

[141] Histopathologische Diagnostik chronisch entzündlicher Darmerkrankungen. Historischer Rückblick und aktuelle Übersicht. Pathologe 1999; 20: $276-287$ 
[142] Gomes P, du Boulay C, Smith CL et al. Relationship between disease activity indices and colonoscopic findings in patients with colonic inflammatory bowel disease. Gut 1986; 27: 92-95

[143] D'Haens G, Van Deventer S, Van Hogezand R et al. Endoscopic and histological healing with infliximab anti-tumor necrosis factor antibodies in Crohn's disease: A European multicenter trial. Gastroenterology 1999; 116: 1029-1034

[144] Riley SA, Mani V, Goodman MJ et al. Microscopic activity in ulcerative colitis: what does it mean? Gut 1991; 32: 174-178

[145] Bitton A, Peppercorn MA, Antonioli DA et al. Clinical, biological, and histologic parameters as predictors of relapse in ulcerative colitis. Gastroenterology 2001; 120: 13-20

[146] Nishio Y, Ando T, Maeda O et al. Pit patterns in rectal mucosa assessed by magnifying colonoscope are predictive of relapse in patients with quiescent ulcerative colitis. Gut 2006; 55: 1768 - 1773

[147] Marchal-Bressenot A, Salleron J, Boulagnon-Rombi C et al. Development and validation of the Nancy histological index for UC. Gut 2017; 66: $43-49$

[148] Mosli MH, Feagan BG, Zou G et al. Development and validation of a histological index for UC. Gut 2017; 66: 50 - 58

[149] Riddell RH, Goldman H, Ransohoff DF et al. Dysplasia in inflammatory bowel disease: standardized classification with provisional clinical applications. Hum Pathol 1983; 14: 931 - 968

[150] Goldman H. Significance and detection of dysplasia in chronic colitis. Cancer 1996; 78: $2261-2263$

[151] Pohl C, Hombach A, Kruis W. Chronic inflammatory bowel disease and cancer. Hepatogastroenterology 2000; 47: 57-70

[152] Melville DM, Jass JR, Morson BC et al. Observer study of the grading of dysplasia in ulcerative colitis: comparison with clinical outcome. Hum Pathol 1989; 20: 1008-1014

[153] Eaden J, Abrams K, McKay H et al. Inter-observer variation between general and specialist gastrointestinal pathologists when grading dysplasia in ulcerative colitis. J Pathol 2001; 194: $152-157$

[154] Odze RD, Goldblum J, Noffsinger A et al. Interobserver variability in the diagnosis of ulcerative colitis-associated dysplasia by telepathology. Mod Pathol 2002; 15: 379-386

[155] Dignass A, Eliakim R, Magro F et al. Second European evidence-based consensus on the diagnosis and management of ulcerative colitis part 1: definitions and diagnosis. J Crohns Colitis 2012; 6: 965 - 990

[156] Magro F, Gionchetti P, Eliakim R et al. Third European Evidence-based Consensus on Diagnosis and Management of Ulcerative Colitis. Part 1: Definitions, Diagnosis, Extra-intestinal Manifestations, Pregnancy, Cancer Surveillance, Surgery, and Ileo-anal Pouch Disorders. J Crohns Colitis 2017; 11: 649-670

[157] Laine L, Kaltenbach T, Barkun A et al. SCENIC international consensus statement on surveillance and management of dysplasia in inflammatory bowel disease. Gastrointest Endosc 2015; 81: 489-501 e26

[158] Farraye FA, Odze RD, Eaden J et al. AGA medical position statement on the diagnosis and management of colorectal neoplasia in inflammatory bowel disease. Gastroenterology 2010; 138: 738 - 745

[159] Rutter MD, Saunders BP, Wilkinson KH et al. Most dysplasia in ulcerative colitis is visible at colonoscopy. Gastrointest Endosc 2004; 60: $334-339$

[160] Blackstone MO, Riddell RH, Rogers BH et al. Dysplasia-associated lesion or mass (DALM) detected by colonoscopy in long-standing ulcerative colitis: an indication for colectomy. Gastroenterology 1981; 80: $366-374$

[161] Lennard-Jones JE, Melville DM, Morson BC et al. Precancer and cancer in extensive ulcerative colitis: findings among 401 patients over 22 years. Gut 1990; 31: $800-806$
[162] Butt JH, Konishi F, Morson BC et al. Macroscopic lesions in dysplasia and carcinoma complicating ulcerative colitis. Dig Dis Sci 1983; 28: $18-26$

[163] Jonsson B, Ahsgren L, Andersson LO et al. Colorectal cancer surveillance in patients with ulcerative colitis. Br J Surg 1994; 81: 689-691

[164] Nugent FW, Haggitt RC, Gilpin PA. Cancer surveillance in ulcerative colitis. Gastroenterology 1991; 100: 1241 - 1248

[165] Lofberg R, Brostrom O, Karlen P et al. Colonoscopic surveillance in long-standing total ulcerative colitis-a 15-year follow-up study. Gastroenterology 1990; 99: 1021 - 1031

[166] Eaden J, Abrams K, Ekbom A et al. Colorectal cancer prevention in ulcerative colitis: a case-control study. Aliment Pharmacol Ther 2000; 14: $145-153$

[167] Rosenstock E, Farmer RG, Petras R et al. Surveillance for colonic carcinoma in ulcerative colitis. Gastroenterology 1985; 89: 1342-1346

[168] Karlen P, Kornfeld D, Brostrom O et al. Is colonoscopic surveillance reducing colorectal cancer mortality in ulcerative colitis? A population based case control study. Gut 1998; 42: 711 -714

[169] Lashner BA, Turner BC, Bostwick DG et al. Dysplasia and cancer complicating strictures in ulcerative colitis. Dig Dis Sci 1990; 35: 349-352

[170] Choi PM, Nugent FW, Schoetz DJ Jr et al. Colonoscopic surveillance reduces mortality from colorectal cancer in ulcerative colitis. Gastroenterology 1993; 105: $418-424$

[171] Bye WA, Nguyen TM, Parker CE et al. Strategies for detecting colon cancer in patients with inflammatory bowel disease (Review). Cochrane Database Syst Rev 2017; 9: CD000279. doi: 10.1002/14651858. CD000279.pub4

[172] Winther KV, Jess T, Langholz E et al. Long-term risk of cancer in ulcerative colitis: a population-based cohort study from Copenhagen County. Clin Gastroenterol Hepatol 2004; 2: 1088-1095

[173] Lutgens MW, Vleggaar FP, Schipper ME et al. High frequency of early colorectal cancer in inflammatory bowel disease. Gut 2008; 57: 1246 125

[174] Lutgens MW, Oldenburg B, Siersema PD et al. Colonoscopic surveillance improves survival after colorectal cancer diagnosis in inflammatory bowel disease. $\mathrm{Br}$ J Cancer 2009; 101: 1671 - 1675

[175] Eaden JA, Abrams KR, Mayberry JF. The risk of colorectal cancer in ulcerative colitis: a meta-analysis. Gut 2001; 48: 526-535

[176] Lakatos L, Mester G, Erdelyi Z et al. Risk factors for ulcerative colitisassociated colorectal cancer in a Hungarian cohort of patients with ulcerative colitis: results of a population-based study. Inflamm Bowel Dis 2006; 12: $205-211$

[177] Soderlund S, Brandt L, Lapidus A et al. Decreasing time-trends of colorectal cancer in a large cohort of patients with inflammatory bowe disease. Gastroenterology 2009; 136: 1561 -1567; quiz 1818-1819

[178] Gupta RB, Harpaz N, Itzkowitz S et al. Histologic inflammation is a risk factor for progression to colorectal neoplasia in ulcerative colitis: a cohort study. Gastroenterology 2007; 133: 1099 - 1105; quiz 13401341

[179] Jess T, Loftus EV Jr, Velayos FS et al. Risk of intestinal cancer in inflammatory bowel disease: a population-based study from olmsted county, Minnesota. Gastroenterology 2006; 130: 1039-1046

[180] Rutter MD, Saunders BP, Wilkinson KH et al. Thirty-year analysis of a colonoscopic surveillance program for neoplasia in ulcerative colitis. Gastroenterology 2006; 130: 1030-1038

[181] Velayos FS, Loftus EV Jr, Jess T et al. Predictive and protective factors associated with colorectal cancer in ulcerative colitis: A case-control study. Gastroenterology 2006; 130: 1941 - 1949

[182] Jess T, Rungoe C, Peyrin-Biroulet L. Risk of colorectal cancer in patients with ulcerative colitis: a meta-analysis of population-based cohort studies. Clin Gastroenterol Hepatol 2012; 10: 639-645 
[183] Jess T, Horvath-Puho E, Fallingborg J et al. Cancer risk in inflammatory bowel disease according to patient phenotype and treatment: a Danish population-based cohort study. Am J Gastroenterol 2013; 108 : 1869- 1876

[184] Choi CH, Rutter MD, Askari A et al. Forty-Year Analysis of Colonoscopic Surveillance Program for Neoplasia in Ulcerative Colitis: An Updated Overview. Am J Gastroenterol 2015; 110: 1022 - 1034

[185] Selinger CP, Andrews JM, Titman A et al. Long-term follow-up reveals low incidence of colorectal cancer, but frequent need for resection, among Australian patients with inflammatory bowel disease. Clin Gastroenterol Hepatol 2014; 12: 644-650

[186] Annese V, Daperno M, Rutter MD et al. European evidence based consensus for endoscopy in inflammatory bowel disease. J Crohns Colitis 2013; 7: $982-1018$

[187] Thomas-Gibson S, Rogers P, Cooper S et al. Judgement of the quality of bowel preparation at screening flexible sigmoidoscopy is associated with variability in adenoma detection rates. Endoscopy 2006; 38 : $456-460$

[188] Toruner M, Harewood GC, Loftus EV Jr et al. Endoscopic factors in the diagnosis of colorectal dysplasia in chronic inflammatory bowel disease. Inflamm Bowel Dis 2005; 11: 428-434

[189] Van Assche G, Dignass A, Bokemeyer B et al. Second European evidence-based consensus on the diagnosis and management of ulcerative colitis part 3: special situations. J Crohns Colitis 2013; 7: 1-33

[190] Rutter M, Saunders B, Wilkinson K et al. Severity of inflammation is a risk factor for colorectal neoplasia in ulcerative colitis. Gastroenterology 2004; 126: $451-459$

[191] Claessen MM, Lutgens MW, van Buuren HR et al. More right-sided IBD-associated colorectal cancer in patients with primary sclerosing cholangitis. Inflamm Bowel Dis 2009; 15: 1331 - 1336

[192] Cairns SR, Scholefield JH, Steele RJ et al. Guidelines for colorectal cancer screening and surveillance in moderate and high risk groups (update from 2002). Gut 2010; 59: 666-689

[193] Bernstein CN, Shanahan F, Weinstein WM. Are we telling patients the truth about surveillance colonoscopy in ulcerative colitis? Lancet 1994; 343: $71-74$

[194] Befrits R, Ljung T, Jaramillo E et al. Low-grade dysplasia in extensive, long-standing inflammatory bowel disease: a follow-up study. Dis Colon Rectum 2002; 45: 615-620

[195] Leidenius M, Kellokumpu I, Husa A et al. Dysplasia and carcinoma in longstanding ulcerative colitis: an endoscopic and histological surveillance programme. Gut 1991; 32: 1521 - 1525

[196] Jess T, Loftus EV Jr, Velayos FS et al. Incidence and prognosis of colorectal dysplasia in inflammatory bowel disease: a population-based study from Olmsted County, Minnesota. Inflamm Bowel Dis 2006; 12: $669-676$

[197] Thomas T, Abrams KA, Robinson RJ et al. Meta-analysis: cancer risk of low-grade dysplasia in chronic ulcerative colitis. Aliment Pharmacol Ther 2007; 25: 657-668

[198] Lim CH, Dixon MF, Vail A et al. Ten year follow up of ulcerative colitis patients with and without low grade dysplasia. Gut 2003; 52: 1127 1132

[199] Heuschen UA, Hinz U, Allemeyer EH et al. Backwash ileitis is strongly associated with colorectal carcinoma in ulcerative colitis. Gastroenterology 2001; 120: $841-847$

[200] Hlavaty T, Huorka M, Koller T et al. Colorectal cancer screening in patients with ulcerative and Crohn's colitis with use of colonoscopy, chromoendoscopy and confocal endomicroscopy. Eur J Gastroenterol Hepatol 2011; 23: $680-689$

[201] Kiesslich R, Fritsch J, Holtmann M et al. Methylene blue-aided chromoendoscopy for the detection of intraepithelial neoplasia and colon cancer in ulcerative colitis. Gastroenterology 2003; 124: 880-888
[202] Hurlstone DP, Sanders DS, Lobo AJ et al. Indigo carmine-assisted highmagnification chromoscopic colonoscopy for the detection and characterisation of intraepithelial neoplasia in ulcerative colitis: a prospective evaluation. Endoscopy 2005; 37: 1186-1192

[203] Kiesslich R, Goetz M, Lammersdorf K et al. Chromoscopy-guided endomicroscopy increases the diagnostic yield of intraepithelial neoplasia in ulcerative colitis. Gastroenterology 2007; 132: 874-882

[204] Subramanian V, Mannath J, Ragunath K et al. Meta-analysis: the diagnostic yield of chromoendoscopy for detecting dysplasia in patients with colonic inflammatory bowel disease. Aliment Pharmacol Ther 2011; 33: 304-312

[205] Wu L, Li P, Wu J et al. The diagnostic accuracy of chromoendoscopy for dysplasia in ulcerative colitis: meta-analysis of six randomized controlled trials. Colorectal Dis 2012; 14: 416-420

[206] Carballal S, Maisterra S, Lopez-Serrano A et al. Real-life chromoendoscopy for neoplasia detection and characterisation in long-standing IBD. Gut 2018; 67: 70-78

[207] lannone A, Ruospo M, Wong G et al. Chromoendoscopy for Surveillance in Ulcerative Colitis and Crohn's Disease: A Systematic Review of Randomized Trials. Clin Gastroenterol Hepatol 2017; 15: 1684 1697

[208] Gasia MF, Ghosh S, Panaccione R et al. Targeted Biopsies Identify Larger Proportions of Patients With Colonic Neoplasia Undergoing High-Definition Colonoscopy, Dye Chromoendoscopy, or Electronic Virtual Chromoendoscopy. Clin Gastroenterol Hepatol 2016; 14: 704 $712 \mathrm{e} 4$

[209] Watanabe T, Ajioka Y, Mitsuyama K et al. Comparison of Targeted vs Random Biopsies for Surveillance of Ulcerative Colitis-Associated Colorectal Cancer. Gastroenterology 2016; 151: 1122 - 1130

[210] Moussata D, Allez M, Cazals-Hatem D et al. Are random biopsies still useful for the detection of neoplasia in patients with IBD undergoing surveillance colonoscopy with chromoendoscopy? Gut 2018; 67: $616-624$

[211] Subramanian V, Ramappa V, Telakis E et al. Comparison of high definition with standard white light endoscopy for detection of dysplastic lesions during surveillance colonoscopy in patients with colonic inflammatory bowel disease. Inflamm Bowel Dis 2013; 19: 350-355

[212] lannone A, Ruospo M, Wong G et al. Chromoendoscopy for Surveillance in Ulcerative Colitis and Crohn's Disease: A Systematic Review of Randomized Trials. Clin Gastroenterol Hepatol 2017; 15: 1684-1697 e11

[213] Dekker E, van den Broek FJ, Reitsma JB et al. Narrow-band imaging compared with conventional colonoscopy for the detection of dysplasia in patients with longstanding ulcerative colitis. Endoscopy 2007; 39: $216-221$

[214] Ignjatovic A, East JE, Subramanian V et al. Narrow band imaging for detection of dysplasia in colitis: a randomized controlled trial. Am J Gastroenterol 2012; 107: 885-890

[215] Pellise M, Lopez-Ceron M, Rodriguez de Miguel C et al. Narrow-band imaging as an alternative to chromoendoscopy for the detection of dysplasia in long-standing inflammatory bowel disease: a prospective, randomized, crossover study. Gastrointest Endosc 2011; 74: 840 - 848

[216] Gunther U, Kusch D, Heller F et al. Surveillance colonoscopy in patients with inflammatory bowel disease: comparison of random biopsy vs. targeted biopsy protocols. Int J Colorectal Dis 2011; 26: 667-672

[217] van den Broek FJ, van Es JA, van Eeden S et al. Pilot study of probebased confocal laser endomicroscopy during colonoscopic surveillance of patients with longstanding ulcerative colitis. Endoscopy 2011; 43: $116-122$

[218] Bisschops R, Bessissow T, Joseph JA et al. Chromoendoscopy versus narrow band imaging in UC: a prospective randomised controlled trial. Gut 2018; 67: 1087-1094 
[219] Leifeld L, Rogler G, Stallmach A et al. White-Light or Narrow-Band Imaging Colonoscopy in Surveillance of Ulcerative Colitis: A Prospective Multicenter Study. Clin Gastroenterol Hepatol 2015; 13: 1776 1781 e1

[220] Omata F, Ohde S, Deshpande GA et al. Image-enhanced, chromo, and cap-assisted colonoscopy for improving adenoma/neoplasia detection rate: a systematic review and meta-analysis. Scand J Gastroenterol 2014; 49: $222-237$

[221] Taylor BA, Pemberton JH, Carpenter HA et al. Dysplasia in chronic ulcerative colitis: implications for colonoscopic surveillance. Dis Colon Rectum 1992; 35: 950 - 956

[222] Fumery M, Dulai PS, Gupta S et al. Incidence, Risk Factors, and Outcomes of Colorectal Cancer in Patients With Ulcerative Colitis With Low-Grade Dysplasia: A Systematic Review and Meta-analysis. Clin Gastroenterol Hepatol 2017; 15: 665 - 674 e5

[223] Ullman T, Croog V, Harpaz N et al. Progression of flat low-grade dysplasia to advanced neoplasia in patients with ulcerative colitis. Gastroenterology 2003; 125: 1311 -1319

[224] Rubin PH, Friedman S, Harpaz N et al. Colonoscopic polypectomy in chronic colitis: conservative management after endoscopic resection of dysplastic polyps. Gastroenterology 1999; 117: 1295-1300

[225] Vieth M, Behrens H, Stolte M. Sporadic adenoma in ulcerative colitis: endoscopic resection is an adequate treatment. Gut 2006; 55: 1151 1155

[226] Odze RD, Farraye FA, Hecht JL et al. Long-term follow-up after polypectomy treatment for adenoma-like dysplastic lesions in ulcerative colitis. Clin Gastroenterol Hepatol 2004; 2: 534- 541

[227] Wanders LK, Dekker E, Pullens B et al. Cancer risk after resection of polypoid dysplasia in patients with longstanding ulcerative colitis: a meta-analysis. Clin Gastroenterol Hepatol 2014; 12: 756 - 764

[228] Pardi DS, Loftus EV Jr, Kremers WK et al. Ursodeoxycholic acid as a chemopreventive agent in patients with ulcerative colitis and primary sclerosing cholangitis. Gastroenterology 2003; 124: 889-893

[229] Tung BY, Emond M], Haggitt RC et al. Ursodiol use is associated with lower prevalence of colonic neoplasia in patients with ulcerative colitis and primary sclerosing cholangitis. Ann Intern Med 2001; 134: 89-95

[230] Lindor KD, Kowdley KV, Luketic VA et al. High-dose ursodeoxycholic acid for the treatment of primary sclerosing cholangitis. Hepatology 2009; 50: $808-814$

[231] LeBlanc K, Mosli MH, Parker CE et al. The impact of biological interventions for ulcerative colitis on health-related quality of life. Cochrane Database Syst Rev 2015; 22: CD008655

[232] Ford AC, Achkar JP, Khan KJ et al. Efficacy of 5-aminosalicylates in ulcerative colitis: systematic review and meta-analysis. Am J Gastroenterol 2011; 106: $601-616$

[233] Timmer A, Patton PH, Chande $\mathrm{N}$ et al. Azathioprine and 6-mercaptopurine for maintenance of remission in ulcerative colitis. Cochrane Database Syst Rev 2016; 18: CD000478

[234] Colombel JF, Rutgeerts P, Reinisch W et al. Early mucosal healing with infliximab is associated with improved long-term clinical outcomes in ulcerative colitis. Gastroenterology 2011; 141: 1194-1201

[235] Marshall JK, Thabane M, Steinhart AH et al. Rectal 5-aminosalicylic acid for induction of remission in ulcerative colitis. Cochrane Database Syst Rev 2010; 20: CD004115

[236] Lie MR, Kanis SL, Hansen BE et al. Drug therapies for ulcerative proctitis: systematic review and meta-analysis. Inflamm Bowel Dis 2014; 20: $2157-2178$

[237] Cohen RD, Dalal SR. Systematic Review: Rectal Therapies for the Treatment of Distal Forms of Ulcerative Colitis. Inflamm Bowel Dis 2015; 21: $1719-1736$
[238] Romkens TE, Kampschreur MT, Drenth JP et al. High mucosal healing rates in 5-ASA-treated ulcerative colitis patients: results of a meta-analysis of clinical trials. Inflamm Bowel Dis 2012; 18: 2190-2198

[239] Andus T, Kocjan A, Muser M et al. Clinical trial: a novel high-dose $1 \mathrm{~g}$ mesalamine suppository (Salofalk) once daily is as efficacious as a 500-mg suppository thrice daily in active ulcerative proctitis. Inflamm Bowel Dis 2010; 16: 1947-1956

[240] Lamet M. A multicenter, randomized study to evaluate the efficacy and safety of mesalamine suppositories $1 \mathrm{~g}$ at bedtime and $500 \mathrm{mg}$ Twice daily in patients with active mild-to-moderate ulcerative proctitis. Dig Dis Sci 2011; 56: 513-522

[241] Gionchetti P, Rizzello F, Venturi A et al. Comparison of oral with rectal mesalazine in the treatment of ulcerative proctitis. Dis Colon Rectum 1998; 41: $93-97$

[242] Marshall JK, Irvine E]. Rectal corticosteroids versus alternative treatments in ulcerative colitis: a meta-analysis. Gut 1997; 40: 775-781

[243] Regueiro M, Loftus EV Jr, Steinhart AH et al. Medical management of left-sided ulcerative colitis and ulcerative proctitis: critical evaluation of therapeutic trials. Inflamm Bowel Dis 2006; 12: 979-994

[244] Mulder C], Fockens P, Meijer JW et al. Beclomethasone dipropionate $(3 \mathrm{mg}$ ) versus 5 -aminosalicylic acid $(2 \mathrm{~g})$ versus the combination of both ( $3 \mathrm{mg} / 2 \mathrm{~g}$ ) as retention enemas in active ulcerative proctitis. Eur J Gastroenterol Hepatol 1996; 8: 549-553

[245] Sandborn W], Bosworth B, Zakko S et al. Budesonide foam induces remission in patients with mild to moderate ulcerative proctitis and ulcerative proctosigmoiditis. Gastroenterology 2015; 148: 740 - 750 e2

[246] Bosworth BP, Sandborn W], Rubin DT et al. Baseline Oral 5-ASA Use and Efficacy and Safety of Budesonide Foam in Patients with Ulcerative Proctitis and Ulcerative Proctosigmoiditis: Analysis of 2 Phase 3 Studies. Inflamm Bowel Dis 2016; 22: 1881 - 1886

[247] Leifeld L, Pfutzer R, Morgenstern J et al. Mesalazine granules are superior to Eudragit-L-coated mesalazine tablets for induction of remission in distal ulcerative colitis - a pooled analysis. Aliment Pharmacol Ther 2011; 34: 1115-1122

[248] Benson A, Barrett T, Sparberg M et al. Efficacy and safety of tacrolimus in refractory ulcerative colitis and Crohn's disease: a single-center experience. Inflamm Bowel Dis 2008; 14: 7-12

[249] Lawrance IC, Baird A, Lightower D et al. Efficacy of Rectal Tacrolimus for Induction Therapy in Patients With Resistant Ulcerative Proctitis. Clin Gastroenterol Hepatol 2017; 15: 1248 - 1255

[250] Ford AC, Khan KJ, Achkar JP et al. Efficacy of oral vs. topical, or combined oral and topical 5-aminosalicylates, in Ulcerative Colitis: systematic review and meta-analysis. Am J Gastroenterol 2012; 107: 167 - 176; author reply 177

[251] Pimpo MT, Galletti B, Palumbo G et al. Mesalazine vanishing time from rectal mucosa following its topical administration. J Crohns Colitis 2010; 4: $102-105$

[252] Cortot A, Maetz D, Degoutte E et al. Mesalamine foam enema versus mesalamine liquid enema in active left-sided ulcerative colitis. Am J Gastroenterol 2008; 103: $3106-3114$

[253] Wang Y, Parker CE, Bhanji T et al. Oral 5-aminosalicylic acid for induction of remission in ulcerative colitis. Cochrane Database Syst Rev 2016; 4: CD000543

[254] Feagan BG, Chande N, MacDonald JK. Are there any differences in the efficacy and safety of different formulations of Oral 5-ASA used for induction and maintenance of remission in ulcerative colitis? evidence from cochrane reviews. Inflamm Bowel Dis 2013; 19: 2031 - 2040

[255] Manguso F, Balzano A. Meta-analysis: the efficacy of rectal beclomethasone dipropionate vs. 5-aminosalicylic acid in mild to moderate distal ulcerative colitis. Aliment Pharmacol Ther 2007; 26: 21 - 29 
[256] Severs M, Mangen MJ, Fidder HH et al. Clinical Predictors of Future Nonadherence in Inflammatory Bowel Disease. Inflamm Bowel Dis 2017; 23: $1568-1576$

[257] Flourie B, Hagege H, Tucat G et al. Randomised clinical trial: once- vs. twice-daily prolonged-release mesalazine for active ulcerative colitis. Aliment Pharmacol Ther 2013; 37: 767-775

[258] Feagan BG, MacDonald JK. Once daily oral mesalamine compared to conventional dosing for induction and maintenance of remission in ulcerative colitis: a systematic review and meta-analysis. Inflamm Bowel Dis 2012; 18: 1785 - 1794

[259] Orchard TR, van der Geest SA, Travis SP. Randomised clinical trial: early assessment after 2 weeks of high-dose mesalazine for moderately active ulcerative colitis - new light on a familiar question. Aliment Pharmacol Ther 2011; 33: 1028-1035

[260] Sherlock ME, Seow CH, Steinhart AH et al. Oral budesonide for induction of remission in ulcerative colitis. Cochrane Database Syst Rev 2010; 6: CD007698

[261] Sandborn W], Travis S, Moro L et al. Once-daily budesonide MMX(R) extended-release tablets induce remission in patients with mild to moderate ulcerative colitis: results from the CORE I study. Gastroenterology 2012; 143: 1218-1226 e1-2

[262] Travis SP, Danese S, Kupcinskas L et al. Once-daily budesonide MMX in active, mild-to-moderate ulcerative colitis: results from the randomised CORE II study. Gut 2014; 63: $433-441$

[263] Sandborn W], Danese S, D’Haens G et al. Induction of clinical and colonoscopic remission of mild-to-moderate ulcerative colitis with budesonide MMX $9 \mathrm{mg}$ : pooled analysis of two phase 3 studies. Aliment Pharmacol Ther 2015; 41: 409-418

[264] Sherlock ME, MacDonald JK, Griffiths AM et al. Oral budesonide for induction of remission in ulcerative colitis. Cochrane Database Syst Rev 2015; 26: CD007698

[265] Marshall JK, Thabane M, Steinhart AH et al. Rectal 5-aminosalicylic acid for maintenance of remission in ulcerative colitis. Cochrane Database Syst Rev 2012; 11: CD004118

[266] Moody GA, Eaden JA, Helyes Z et al. Oral or rectal administration of drugs in IBD? Aliment Pharmacol Ther 1997; 11: 999-1000

[267] Chaparro M, Gisbert JP. Maintenance therapy options for ulcerative colitis. Expert Opin Pharmacother 2016; 17: 1339-1349

[268] Losurdo G, lannone A, Contaldo A et al. Escherichia coli Nissle 1917 in Ulcerative Colitis Treatment: Systematic Review and Meta-analysis. J Gastrointestin Liver Dis 2015; 24: 499-505

[269] Scaldaferri F, Gerardi V, Mangiola F et al. Role and mechanisms of action of Escherichia coli Nissle 1917 in the maintenance of remission in ulcerative colitis patients: An update. World J Gastroenterol 2016; 22: 5505- 5511

[270] d'Albasio G, Pacini F, Camarri E et al. Combined therapy with 5-aminosalicylic acid tablets and enemas for maintaining remission in ulcerative colitis: a randomized double-blind study. Am J Gastroenterol 1997; 92: $1143-1147$

[271] Yokoyama H, Takagi S, Kuriyama S et al. Effect of weekend 5-aminosalicylic acid (mesalazine) enema as maintenance therapy for ulcerative colitis: results from a randomized controlled study. Inflamm Bowel Dis 2007; 13: $1115-1120$

[272] Piodi LP, Ulivieri FM, Cermesoni L et al. Long-term intermittent treatment with low-dose 5-aminosalicylic enemas is efficacious for remission maintenance in ulcerative colitis. Scand J Gastroenterol 2004; 39 : $154-157$

[273] Bokemeyer B, Hommes D, Gill I et al. Mesalazine in left-sided ulcerative colitis: efficacy analyses from the PODIUM trial on maintenance of remission and mucosal healing. J Crohns Colitis 2012; 6: 476-482

[274] Kamm MA, Lichtenstein GR, Sandborn WJ et al. Randomised trial of once- or twice-daily MMX mesalazine for maintenance of remission in ulcerative colitis. Gut 2008; 57: 893-902
[275] Feagan BG, Macdonald JK. Oral 5-aminosalicylic acid for maintenance of remission in ulcerative colitis. Cochrane Database Syst Rev 2012; 10 : CD000544

[276] Dignass AU, Bokemeyer B, Adamek H et al. Mesalamine once daily is more effective than twice daily in patients with quiescent ulcerative colitis. Clin Gastroenterol Hepatol 2009; 7: 762 - 769

[277] Gordon GL, Zakko S, Murthy U et al. Once-daily Mesalamine Formulation for Maintenance of Remission in Ulcerative Colitis: A Randomized, Placebo-controlled Clinical Trial. J Clin Gastroenterol 2016; 50: 318 325

[278] Kruis W, Jonaitis L, Pokrotnieks J et al. Randomised clinical trial: a comparative dose-finding study of three arms of dual release mesalazine for maintaining remission in ulcerative colitis. Aliment Pharmacol Ther 2011; 33: $313-322$

[279] Sandborn W], Korzenik ], Lashner B et al. Once-daily dosing of delayedrelease oral mesalamine (400-mg tablet) is as effective as twice-daily dosing for maintenance of remission of ulcerative colitis. Gastroenterology 2010; 138: 1286-1296, 1296 e1-3

[280] Heyman MB, Kierkus ], Spenard J et al. Efficacy and safety of mesalamine suppositories for treatment of ulcerative proctitis in children and adolescents. Inflamm Bowel Dis 2010; 16: 1931 - 1939

[281] Kobayashi K, Hirai F, Naganuma M et al. A randomized clinical trial of mesalazine suppository: the usefulness and problems of central review of evaluations of colonic mucosal findings. J Crohns Colitis 2014; 8: $1444-1453$

[282] Watanabe M, Nishino H, Sameshima Y et al. Randomised clinical trial: evaluation of the efficacy of mesalazine (mesalamine) suppositories in patients with ulcerative colitis and active rectal inflammation - a placebo-controlled study. Aliment Pharmacol Ther 2013; 38: 264-273

[283] Kruis W, Jonaitis L, Pokrotnieks J et al. Randomised clinical trial: a comparative dose-finding study of three arms of dual release mesalazine for maintaining remission in ulcerative colitis. Aliment Pharmacol Ther 2011; 33: 313-322

[284] Yokoyama H, Takagi S, Kuriyama S et al. Effect of weekend 5-aminosalicylic acid (mesalazine) enema as maintenance therapy for ulcerative colitis: results from a randomized controlled study. Inflamm Bowel Dis 2007; 13: $1115-1120$

[285] Ardizzone S, Porro GB. How long is it advisable to prolong maintenance treatment of patients with ulcerative colitis? Inflamm Bowel Dis 2008; 14 (Suppl. 2): S238-\$239

[286] Heap GA, So K, Weedon M et al. Clinical Features and HLA Association of 5-Aminosalicylate (5-ASA)-induced Nephrotoxicity in Inflammatory Bowel Disease. J Crohns Colitis 2016; 10: 149-158

[287] Zallot C, Billioud V, Frimat L et al. 5-Aminosalicylates and renal function monitoring in inflammatory bowel disease: a nationwide survey. J Crohns Colitis 2013; 7: 551 - 555

[288] Nikolaus S, Schreiber S, Siegmund B et al. Patient Education in a 14-month Randomised Trial Fails to Improve Adherence in Ulcerative Colitis: Influence of Demographic and Clinical Parameters on Non-adherence. J Crohns Colitis 2017; 11: 1052 - 1062

[289] Velayos FS, Terdiman JP, Walsh JM. Effect of 5-aminosalicylate use on colorectal cancer and dysplasia risk: a systematic review and metaanalysis of observational studies. Am J Gastroenterol 2005; 100 : $1345-1353$

[290] Biancone L, Michetti P, Travis S et al. European evidence-based Consensus on the management of ulcerative colitis: Special situations. J Crohns Colitis 2008; 2: 63-92

[291] Rubin DT, Huo D, Kinnucan JA et al. Inflammation is an independent risk factor for colonic neoplasia in patients with ulcerative colitis: a case-control study. Clin Gastroenterol Hepatol 2013; 11: 1601 - 1608 e1-4 
[292] Mathy C, Schneider K, Chen YY et al. Gross versus microscopic pancolitis and the occurrence of neoplasia in ulcerative colitis. Inflamm Bowel Dis 2003; 9: 351 - 355

[293] Choi CH, Ignjatovic-Wilson A, Askari A et al. Low-grade dysplasia in ulcerative colitis: risk factors for developing high-grade dysplasia or colorectal cancer. Am J Gastroenterol 2015; 110: 1461 - 1471; quiz 1472

[294] Lindberg BU, Broome U, Persson B. Proximal colorectal dysplasia or cancer in ulcerative colitis. The impact of primary sclerosing cholangitis and sulfasalazine: results from a 20 -year surveillance study. Dis Colon Rectum 2001; 44: 77 - 85

[295] Itzkowitz SH, Harpaz N. Diagnosis and management of dysplasia in patients with inflammatory bowel diseases. Gastroenterology 2004; 126: $1634-1648$

[296] Lyakhovich A, Gasche C. Systematic review: molecular chemoprevention of colorectal malignancy by mesalazine. Aliment Pharmacol Ther 2010; 31: $202-209$

[297] Ullman T, Croog V, Harpaz N et al. Progression to colorectal neoplasia in ulcerative colitis: effect of mesalamine. Clin Gastroenterol Hepatol 2008; 6: 1225-1230; quiz 1177

[298] Bergeron VNLI, Vienne A, Seksik P et al. Azathioprine [AZA] is associated with less histological inflammation of the colon in inactive IBD. Gastroenterology 2010; 138: 693

[299] Lashner BA, Provencher KS, Seidner DL et al. The effect of folic acid supplementation on the risk for cancer or dysplasia in ulcerative colitis. Gastroenterology 1997; 112: 29-32

[300] Terdiman JP, Steinbuch M, Blumentals WA et al. 5-Aminosalicylic acid therapy and the risk of colorectal cancer among patients with inflammatory bowel disease. Inflamm Bowel Dis 2007; 13: 367 - 371

[301] Matula S, Croog V, Itzkowitz S et al. Chemoprevention of colorectal neoplasia in ulcerative colitis: the effect of 6-mercaptopurine. Clin Gastroenterol Hepatol 2005; 3: 1015-1021

[302] van Schaik FD, van Oijen MG, Smeets HM et al. Thiopurines prevent advanced colorectal neoplasia in patients with inflammatory bowel disease. Gut 2012; 61: 235-240

[303] Beaugerie L, Svrcek M, Seksik P et al. Risk of colorectal high-grade dysplasia and cancer in a prospective observational cohort of patients with inflammatory bowel disease. Gastroenterology 2013; 145: 166 175 e8

[304] Lindgren S, Lofberg R, Bergholm L et al. Effect of budesonide enema on remission and relapse rate in distal ulcerative colitis and proctitis. Scand J Gastroenterol 2002; 37: 705-710

[305] Meyers S, Lerer PK, Feuer E] et al. Predicting the outcome of corticoid therapy for acute ulcerative colitis. Results of a prospective, randomized, double-blind clinical trial. J Clin Gastroenterol 1987; 9: 50 - 54

[306] Lichtenstein GR, Feagan BG, Cohen RD et al. Serious infection and mortality in patients with Crohn's disease: more than 5 years of followup in the TREAT registry. Am J Gastroenterol 2012; 107: 1409-1422

[307] Edwards FC, Truelove SC. The Course and Prognosis of Ulcerative Colitis. Gut 1963; 4: 299-315

[308] Van Assche G, Vermeire S, Rutgeerts P. Management of acute severe ulcerative colitis. Gut 2011; 60: 130-133

[309] Turner D, Walsh CM, Steinhart AH et al. Response to corticosteroids in severe ulcerative colitis: a systematic review of the literature and a meta-regression. Clin Gastroenterol Hepatol 2007; 5: $103-110$

[310] Lynch RW, Lowe D, Protheroe A et al. Outcomes of rescue therapy in acute severe ulcerative colitis: data from the United Kingdom inflammatory bowel disease audit. Aliment Pharmacol Ther 2013; 38: 935 945

[311] Baron JH, Connell AM, Kanaghinis TG et al. Out-patient treatment of ulcerative colitis. Comparison between three doses of oral prednisone. Br Med J 1962; 2: $441-443$
[312] Lennard-Jones JE, Longmore AJ, Newell AC et al. An assessment of prednisone, salazopyrin, and topical hydrocortisone hemisuccinate used as out-patient treatment for ulcerative colitis. Gut 1960; 1: 217 222

[313] Truelove SC, Jewell DP. Intensive intravenous regimen for severe attacks of ulcerative colitis. Lancet 1974; 1: 1067-1070

[314] Truelove SC, Witts L]. Cortisone in ulcerative colitis; preliminary report on a therapeutic trial. Br Med J 1954; 2: 375 - 378

[315] Truelove SC, Witts L]. Cortisone and corticotrophin in ulcerative colitis Br Med J 1959; 1: $387-394$

[316] Rosenberg W, Ireland A, Jewell DP. High-dose methylprednisolone in the treatment of active ulcerative colitis. J Clin Gastroenterol 1990; 12: $40-41$

[317] Bartels SA, Gardenbroek T], Bos L et al. Prolonged preoperative hospital stay is a risk factor for complications after emergency colectomy for severe colitis. Colorectal Dis 2013; 15: $1392-1398$

[318] Bernstein CN, Ng SC, Lakatos PL et al. A review of mortality and surgery in ulcerative colitis: milestones of the seriousness of the disease. Inflamm Bowel Dis 2013; 19: $2001-2010$

[319] Randall ], Singh B, Warren BF et al. Delayed surgery for acute severe colitis is associated with increased risk of postoperative complications. $\mathrm{Br}$ J Surg 2010; 97: 404-409

[320] Roberts SE, Williams JG, Yeates D et al. Mortality in patients with and without colectomy admitted to hospital for ulcerative colitis and Crohn's disease: record linkage studies. BMJ 2007; 335: 1033

[321] Dignass AU, Gasche C, Bettenworth D et al. European consensus on the diagnosis and management of iron deficiency and anaemia in inflammatory bowel diseases. J Crohns Colitis 2015; 9: 211 - 222

[322] Autenrieth DM, Baumgart DC. Toxic megacolon. Inflamm Bowel Dis 2012; 18: 584-591

[323] Gan SI, Beck PL. A new look at toxic megacolon: an update and review of incidence, etiology, pathogenesis, and management. Am J Gastroenterol 2003; 98: 2363-2371

[324] Kvasnovsky CL, Aujla U, Bjarnason I. Nonsteroidal anti-inflammatory drugs and exacerbations of inflammatory bowel disease. Scand J Gastroenterol 2015; 50: 255-263

[325] Long MD, Barnes EL, Herfarth HH et al. Narcotic use for inflammatory bowel disease and risk factors during hospitalization. Inflamm Bowel Dis 2012; 18: 869-876

[326] Domenech E, Vega R, Ojanguren I et al. Cytomegalovirus infection in ulcerative colitis: a prospective, comparative study on prevalence and diagnostic strategy. Inflamm Bowel Dis 2008; 14: 1373-1379

[327] Kishore J, Ghoshal U, Ghoshal UC et al. Infection with cytomegalovirus in patients with inflammatory bowel disease: prevalence, clinical significance and outcome. J Med Microbiol 2004; 53: 1155-1160

[328] Lee HS, Park SH, Kim SH et al. Risk Factors and Clinical Outcomes Associated with Cytomegalovirus Colitis in Patients with Acute Severe Ulcerative Colitis. Inflamm Bowel Dis 2016; 22: 912-918

[329] Papadakis KA, Tung JK, Binder SW et al. Outcome of cytomegalovirus infections in patients with inflammatory bowel disease. Am J Gastroenterol 2001; 96: 2137-2142

[330] Rahier JF, Magro F, Abreu C et al. Second European evidence-based consensus on the prevention, diagnosis and management of opportunistic infections in inflammatory bowel disease. J Crohns Colitis 2014; 8: $443-468$

[331] Siegmund B. Cytomegalovirus infection associated with inflammatory bowel disease. Lancet Gastroenterol Hepatol 2017; 2: 369 - 376

[332] Berg AM, Kelly CP, Farraye FA. Clostridium difficile infection in the inflammatory bowel disease patient. Inflamm Bowel Dis 2013; 19: 194 204 
[333] Issa M, Ananthakrishnan AN, Binion DG. Clostridium difficile and inflammatory bowel disease. Inflamm Bowel Dis 2008; 14: 1432 - 1442

[334] Jen MH, Saxena S, Bottle A et al. Increased health burden associated with Clostridium difficile diarrhoea in patients with inflammatory bowel disease. Aliment Pharmacol Ther 2011; 33: 1322-1331

[335] Murthy SK, Steinhart AH, Tinmouth J et al. Impact of Clostridium difficile colitis on 5-year health outcomes in patients with ulcerative colitis. Aliment Pharmacol Ther 2012; 36: 1032 - 1039

[336] Negron ME, Barkema HW, Rioux K et al. Clostridium difficile infection worsens the prognosis of ulcerative colitis. Can J Gastroenterol Hepatol 2014; 28 : $373-380$

[337] Nguyen GC, Kaplan GG, Harris ML et al. A national survey of the prevalence and impact of Clostridium difficile infection among hospitalized inflammatory bowel disease patients. Am J Gastroenterol 2008 103: $1443-1450$

[338] Hagel S, Epple HJ, Feurle GE et al. S2k-guideline gastrointestinal infectious diseases and Whipple's disease. Z Gastroenterol 2015; 53: 418 459

[339] Ben-Horin S, Margalit M, Bossuyt P et al. Combination immunomodulator and antibiotic treatment in patients with inflammatory bowel disease and clostridium difficile infection. Clin Gastroenterol Hepatol 2009; 7: $981-987$

[340] Rahier JF, Ben-Horin S, Chowers Y et al. European evidence-based Consensus on the prevention, diagnosis and management of opportunistic infections in inflammatory bowel disease. J Crohns Colitis 2009; 3: $47-91$

[341] Grainge M], West J, Card TR. Venous thromboembolism during active disease and remission in inflammatory bowel disease: a cohort study. Lancet 2010; 375: 657-663

[342] Harbord M, Annese V, Vavricka SR et al. The First European Evidencebased Consensus on Extra-intestinal Manifestations in Inflammatory Bowel Disease. J Crohns Colitis 2016; 10: 239-254

[343] Kappelman MD, Horvath-Puho E, Sandler RS et al. Thromboembolic risk among Danish children and adults with inflammatory bowel diseases: a population-based nationwide study. Gut 2011; 60: 937 - 943

[344] Nguyen GC, Bernstein CN, Bitton A et al. Consensus statements on the risk, prevention, and treatment of venous thromboembolism in inflammatory bowel disease: Canadian Association of Gastroenterology. Gastroenterology 2014; 146: 835 - 848 e6

[345] Gonzalez-Huix F, Fernandez-Banares F, Esteve-Comas M et al. Enteral versus parenteral nutrition as adjunct therapy in acute ulcerative colitis. Am J Gastroenterol 1993; 88: 227 -232

[346] Mclntyre PB, Powell-Tuck J, Wood SR et al. Controlled trial of bowel rest in the treatment of severe acute colitis. Gut 1986; 27: 481 - 485

[347] Turner D, Travis SP, Griffiths AM et al. Consensus for managing acute severe ulcerative colitis in children: a systematic review and joint statement from ECCO, ESPGHAN, and the Porto IBD Working Group of ESPGHAN. Am J Gastroenterol 2011; 106: 574-588

[348] Lindgren SC, Flood LM, Kilander AF et al. Early predictors of glucocorticosteroid treatment failure in severe and moderately severe attacks of ulcerative colitis. Eur J Gastroenterol Hepatol 1998; 10: 831 - 835

[349] Llao J, Naves JE, Ruiz-Cerulla A et al. Intravenous corticosteroids in moderately active ulcerative colitis refractory to oral corticosteroids. J Crohns Colitis 2014; 8: 1523-1528

[350] Rutgeerts P, Sandborn W], Feagan BG et al. Infliximab for induction and maintenance therapy for ulcerative colitis. N Engl J Med 2005; 353 : $2462-2476$

[351] Sandborn W], van Assche G, Reinisch W et al. Adalimumab induces and maintains clinical remission in patients with moderate-to-severe ulcerative colitis. Gastroenterology 2012; 142: 257 - 265 e1-3
[352] Sandborn W], Feagan BG, Marano C et al. Subcutaneous golimumab induces clinical response and remission in patients with moderate-tosevere ulcerative colitis. Gastroenterology 2014; 146: 85 - 95; quiz e14-15

[353] Sandborn W], Feagan BG, Marano C et al. Subcutaneous golimumab maintains clinical response in patients with moderate-to-severe ulcerative colitis. Gastroenterology 2014; 146: 96 - 109 e1

[354] Lennard-Jones JE, Ritchie JK, Hilder W et al. Assessment of severity in colitis: a preliminary study. Gut 1975; 16: 579 - 584

[355] Travis SP, Farrant JM, Ricketts C et al. Predicting outcome in severe ulcerative colitis. Gut 1996; 38: $905-910$

[356] Benazzato L, D’Inca R, Grigoletto F et al. Prognosis of severe attacks in ulcerative colitis: effect of intensive medical treatment. Dig Liver Dis 2004; 36: $461-466$

[357] Chew CN, Nolan DJ, Jewell DP. Small bowel gas in severe ulcerative colitis. Gut 1991; 32: $1535-1537$

[358] Carbonnel F, Gargouri D, Lemann M et al. Predictive factors of outcome of intensive intravenous treatment for attacks of ulcerative colitis. Aliment Pharmacol Ther 2000; 14: 273-279

[359] Corte C, Fernandopulle N, Catuneanu AM et al. Association between the ulcerative colitis endoscopic index of severity (UCEIS) and outcomes in acute severe ulcerative colitis. J Crohns Colitis 2015; 9: 376 381

[360] Ho GT, Mowat C, Goddard C] et al. Predicting the outcome of severe ulcerative colitis: development of a novel risk score to aid early selection of patients for second-line medical therapy or surgery. Aliment Pharmacol Ther 2004; 19: 1079-1087

[361] Kato K, Ohkusa T, Terao S et al. Adjunct antibiotic combination therapy for steroid-refractory or -dependent ulcerative colitis: an open-label multicentre study. Aliment Pharmacol Ther 2014; 39: 949-956

[362] Mantzaris G], Hatzis A, Kontogiannis P et al. Intravenous tobramycin and metronidazole as an adjunct to corticosteroids in acute, severe ulcerative colitis. Am J Gastroenterol 1994; 89: 43-46

[363] Lichtiger S, Present DH, Kornbluth A et al. Cyclosporine in severe ulcerative colitis refractory to steroid therapy. N Engl J Med 1994; 330: $1841-1845$

[364] D’Haens G, Lemmens L, Geboes K et al. Intravenous cyclosporine versus intravenous corticosteroids as single therapy for severe attacks of ulcerative colitis. Gastroenterology 2001; 120: 1323-1329

[365] Van Assche G, D’Haens G, Noman M et al. Randomized, double-blind comparison of $4 \mathrm{mg} / \mathrm{kg}$ versus $2 \mathrm{mg} / \mathrm{kg}$ intravenous cyclosporine in severe ulcerative colitis. Gastroenterology 2003; 125: 1025-1031

[366] Laharie D, Bourreille A, Branche J et al. Ciclosporin versus infliximab in patients with severe ulcerative colitis refractory to intravenous steroids: a parallel, open-label randomised controlled trial. Lancet 2012; 380: $1909-1915$

[367] Williams JG, Alam MF, Alrubaiy L et al. Infliximab versus ciclosporin for steroid-resistant acute severe ulcerative colitis (CONSTRUCT): a mixed methods, open-label, pragmatic randomised trial. Lancet Gastroenterol Hepatol 2016; 1: 15-24

[368] Shibolet O, Regushevskaya E, Brezis M et al. Cyclosporine A for induction of remission in severe ulcerative colitis. Cochrane Database Syst Rev 2005; 25: CD004277

[369] Cohen RD, Stein R, Hanauer SB. Intravenous cyclosporin in ulcerative colitis: a five-year experience. Am J Gastroenterol 1999; 94: 1587 1592

[370] Moskovitz DN, Van Assche G, Maenhout B et al. Incidence of colectomy during long-term follow-up after cyclosporine-induced remission of severe ulcerative colitis. Clin Gastroenterol Hepatol 2006; 4: 760 - 765

[371] Bamba S, Tsujikawa T, Inatomi O et al. Factors affecting the efficacy of cyclosporin A therapy for refractory ulcerative colitis. J Gastroenterol Hepatol 2010; 25: $494-498$ 
[372] Walch A, Meshkat M, Vogelsang $\mathrm{H}$ et al. Long-term outcome in patients with ulcerative colitis treated with intravenous cyclosporine $A$ is determined by previous exposure to thiopurines. J Crohns Colitis 2010; 4: $398-404$

[373] Cheifetz AS, Stern J, Garud S et al. Cyclosporine is safe and effective in patients with severe ulcerative colitis. J Clin Gastroenterol 2011; 45: $107-112$

[374] Ogata H, Matsui T, Nakamura M et al. A randomised dose finding study of oral tacrolimus (FK506) therapy in refractory ulcerative colitis. Gut 2006; 55: $1255-1262$

[375] Ogata H, Kato J, Hirai F et al. Double-blind, placebo-controlled trial of oral tacrolimus (FK506) in the management of hospitalized patients with steroid-refractory ulcerative colitis. Inflamm Bowel Dis 2012; 18: $803-808$

[376] Komaki Y, Komaki F, Ido A et al. Efficacy and Safety of Tacrolimus Therapy for Active Ulcerative Colitis; A Systematic Review and Meta-analysis. J Crohns Colitis 2016; 10: 484-494

[377] Schmidt KJ, Herrlinger KR, Emmrich J et al. Short-term efficacy of tacrolimus in steroid-refractory ulcerative colitis - experience in 130 patients. Aliment Pharmacol Ther 2013; 37: 129-136

[378] Yamamoto T, Shimoyama T, Umegae S et al. Tacrolimus vs. anti-tumour necrosis factor agents for moderately to severely active ulcerative colitis: a retrospective observational study. Aliment Pharmacol Ther 2016; 43: 705-716

[379] Jarnerot G, Hertervig E, Friis-Liby I et al. Infliximab as rescue therapy in severe to moderately severe ulcerative colitis: a randomized, placebocontrolled study. Gastroenterology 2005; 128: 1805-1811

[380] Gustavsson A, Jarnerot G, Hertervig E et al. Clinical trial: colectomy after rescue therapy in ulcerative colitis - 3-year follow-up of the Swedish-Danish controlled infliximab study. Aliment Pharmacol Ther 2010; 32: 984-989

[381] Sjoberg M, Magnuson A, Bjork J et al. Infliximab as rescue therapy in hospitalised patients with steroid-refractory acute ulcerative colitis: a long-term follow-up of 211 Swedish patients. Aliment Pharmacol Ther 2013; 38: $377-387$

[382] Monterubbianesi R, Aratari A, Armuzzi A et al. Infliximab three-dose induction regimen in severe corticosteroid-refractory ulcerative colitis: early and late outcome and predictors of colectomy. J Crohns Colitis 2014; 8: $852-858$

[383] Mortensen C, Caspersen S, Christensen NL et al. Treatment of acute ulcerative colitis with infliximab, a retrospective study from three Danish hospitals. J Crohns Colitis 2011; 5: 28-33

[384] Brandse JF, Mathot RA, van der Kleij D et al. Pharmacokinetic Features and Presence of Antidrug Antibodies Associate With Response to Infliximab Induction Therapy in Patients With Moderate to Severe UIcerative Colitis. Clin Gastroenterol Hepatol 2016; 14: 251 - 258 e1-2

[385] Brandse JF, van den Brink GR, Wildenberg ME et al. Loss of Infliximab Into Feces Is Associated With Lack of Response to Therapy in Patients With Severe Ulcerative Colitis. Gastroenterology 2015; 149: 350 - 355 e2

[386] Gibson DJ, Heetun ZS, Redmond CE et al. An accelerated infliximab induction regimen reduces the need for early colectomy in patients with acute severe ulcerative colitis. Clin Gastroenterol Hepatol 2015; 13: $330-335$ e1

[387] Armuzzi A, Pugliese D, Danese S et al. Long-term combination therapy with infliximab plus azathioprine predicts sustained steroid-free clinical benefit in steroid-dependent ulcerative colitis. Inflamm Bowel Dis 2014; 20: $1368-1374$

[388] Chang KH, Burke JP, Coffey JC. Infliximab versus cyclosporine as rescue therapy in acute severe steroid-refractory ulcerative colitis: a systematic review and meta-analysis. Int J Colorectal Dis 2013; 28: 287 293
[389] Lowenberg M, Duijvis NW, Ponsioen C et al. Length of hospital stay and associated hospital costs with infliximab versus cyclosporine in severe ulcerative colitis. Eur J Gastroenterol Hepatol 2014; 26: 1240 - 1246

[390] Hyde GM, Jewell DP, Kettlewell MG et al. Cyclosporin for severe ulcerative colitis does not increase the rate of perioperative complications. Dis Colon Rectum 2001; 44: 1436 - 1440

[391] Ferrante M, D’Hoore A, Vermeire $S$ et al. Corticosteroids but not infliximab increase short-term postoperative infectious complications in patients with ulcerative colitis. Inflamm Bowel Dis 2009; 15: 1062 1070

[392] Narula N, Fine M, Colombel JF et al. Systematic Review: Sequential Rescue Therapy in Severe Ulcerative Colitis: Do the Benefits Outweigh the Risks? Inflamm Bowel Dis 2015; 21: 1683 - 1694

[393] Hebden JM, Blackshaw PE, Perkins AC et al. Limited exposure of the healthy distal colon to orally-dosed formulation is further exaggerated in active left-sided ulcerative colitis. Aliment Pharmacol Ther 2000; 14 : $155-161$

[394] Nel JA. Management of patients with AMS 800 urinary artificial sphincter. Nurs RSA 1991; 6: 28-32

[395] Sandborn W], Tremaine WJ, Schroeder KW et al. A placebo-controlled trial of cyclosporine enemas for mildly to moderately active left-sided ulcerative colitis. Gastroenterology 1994; 106: 1429-1435

[396] Scheppach W. Treatment of distal ulcerative colitis with short-chain fatty acid enemas. A placebo-controlled trial. German-Austrian SCFA Study Group. Dig Dis Sci 1996; 41: 2254-2259

[397] van Dieren JM, van Bodegraven AA, Kuipers EJ et al. Local application of tacrolimus in distal colitis: feasible and safe. Inflamm Bowel Dis 2009; 15: $193-198$

[398] Carbonnel F, Boruchowicz A, Duclos B et al. Intravenous cyclosporine in attacks of ulcerative colitis: short-term and long-term responses. Dig Dis Sci 1996; 41: 2471 - 2476

[399] Actis GC, Fadda M, David E et al. Colectomy rate in steroid-refractory colitis initially responsive to cyclosporin: a long-term retrospective cohort study. BMC Gastroenterol 2007; 7: 13

[400] Szanto K, Molnar T, Farkas K. New promising combo therapy in inflammatory bowel diseases refractory to anti-TNF agents: cyclosporine plus vedolizumab. J Crohns Colitis 2018; 12: 629

[401] Ardizzone S, Maconi G, Russo A et al. Randomised controlled trial of azathioprine and 5-aminosalicylic acid for treatment of steroid dependent ulcerative colitis. Gut 2006; 55: 47-53

[402] Chebli LA, Chaves LD, Pimentel FF et al. Azathioprine maintains longterm steroid-free remission through 3 years in patients with steroiddependent ulcerative colitis. Inflamm Bowel Dis 2010; 16: 613-619

[403] Panaccione R, Ghosh S, Middleton S et al. Combination therapy with infliximab and azathioprine is superior to monotherapy with either agent in ulcerative colitis. Gastroenterology 2014; 146: $392-400$ e3

[404] Feagan BG, Rutgeerts P, Sands BE et al. Vedolizumab as induction and maintenance therapy for ulcerative colitis. N Engl J Med 2013; 369: $699-710$

[405] Carbonnel F, Colombel JF, Filippi ] et al. Methotrexate Is Not Superior to Placebo for Inducing Steroid-Free Remission, but Induces Steroid-Free Clinical Remission in a Larger Proportion of Patients With Ulcerative Colitis. Gastroenterology 2016; 150: 380 - 388 e4

[406] Lawson MM, Thomas AG, Akobeng AK. Tumour necrosis factor alpha blocking agents for induction of remission in ulcerative colitis. Cochrane Database Syst Rev 2006; 19: CD005112

[407] Reinisch W, Sandborn WJ, Hommes DW et al. Adalimumab for induction of clinical remission in moderately to severely active ulcerative colitis: results of a randomised controlled trial. Gut 2011; 60: 780-787 
[408] Reinisch W, Sandborn W], Panaccione R et al. 52-week efficacy of adalimumab in patients with moderately to severely active ulcerative colitis who failed corticosteroids and/or immunosuppressants. Inflamm Bowel Dis 2013; 19: 1700 - 1709

[409] Gies N, Kroeker KI, Wong K et al. Treatment of ulcerative colitis with adalimumab or infliximab: long-term follow-up of a single-centre cohort. Aliment Pharmacol Ther 2010; 32: 522-528

[410] Colombel JF, Sandborn W], Reinisch W et al. Infliximab, azathioprine, or combination therapy for Crohn's disease. N Engl J Med 2010; 362: $1383-1395$

[411] van Schaik T, Maljaars JP, Roopram RK et al. Influence of combination therapy with immune modulators on anti-TNF trough levels and antibodies in patients with IBD. Inflamm Bowel Dis 2014; 20: $2292-2298$

[412] Yamamoto S, Nakase H, Mikami S et al. Long-term effect of tacrolimus therapy in patients with refractory ulcerative colitis. Aliment Pharmacol Ther 2008; 28: 589-597

[413] Gisbert JP, Marin AC, McNicholl AG et al. Systematic review with metaanalysis: the efficacy of a second anti-TNF in patients with inflammatory bowel disease whose previous anti-TNF treatment has failed. Aliment Pharmacol Ther 2015; 41: 613-623

[414] Baumgart DC, Bokemeyer B, Drabik A et al. Vedolizumab induction therapy for inflammatory bowel disease in clinical practice-a nationwide consecutive German cohort study. Aliment Pharmacol Ther 2016; 43: $1090-1102$

[415] Sandborn WS, D'Haens G. Efficacy and safety of oral tofacitinib as induction therapy in patients with moderate to severe ulcerative colitis: results from two phase 3 randomized controlled trials. Gastroenterology 2016: S157

[416] Sandborn W], Feagan BG, Wolf DC et al. Ozanimod Induction and Maintenance Treatment for Ulcerative Colitis. N Engl J Med 2016; 374: $1754-1762$

[417] Yoo DH, Hrycaj P, Miranda P et al. A randomised, double-blind, parallel-group study to demonstrate equivalence in efficacy and safety of CT-P13 compared with innovator infliximab when coadministered with methotrexate in patients with active rheumatoid arthritis: the PLANETRA study. Ann Rheum Dis 2013; 72: 1613-1620

[418] Park W, Hrycaj P, Jeka S et al. A randomised, double-blind, multicentre, parallel-group, prospective study comparing the pharmacokinetics, safety, and efficacy of CT-P13 and innovator infliximab in patients with ankylosing spondylitis: the PLANETAS study. Ann Rheum Dis 2013; 72: $1605-1612$

[419] Jorgensen KK, Olsen IC, Goll GL et al. Switching from originator infliximab to biosimilar CT-P13 compared with maintained treatment with originator infliximab (NOR-SWITCH): a 52-week, randomised, doubleblind, non-inferiority trial. Lancet 2017; 389: 2304-2316

[420] Hawthorne AB, Logan RF, Hawkey C] et al. Randomised controlled trial of azathioprine withdrawal in ulcerative colitis. BMJ 1992; 305: 20-22

[421] Jewell DP, Truelove SC. Azathioprine in ulcerative colitis: final report on controlled therapeutic trial. Br Med J 1974; 4: 627-630

[422] Sood A, Kaushal V, Midha V et al. The beneficial effect of azathioprine on maintenance of remission in severe ulcerative colitis. J Gastroenterol 2002; 37: $270-274$

[423] Sood A, Midha V, Sood N et al. Azathioprine versus sulfasalazine in maintenance of remission in severe ulcerative colitis. Indian J Gastroenterol 2003; 22: $79-81$

[424] Mate-jimenez J, Hermida C, Cantero-Perona J et al. 6-mercaptopurine or methotrexate added to prednisone induces and maintains remission in steroid-dependent inflammatory bowel disease. Eur J Gastroenterol Hepatol 2000; 12: 1227-1233

[425] Cassinotti A, Actis GC, Duca P et al. Maintenance treatment with azathioprine in ulcerative colitis: outcome and predictive factors after drug withdrawal. Am J Gastroenterol 2009; 104: 2760 - 2767
[426] George J, Present DH, Pou R et al. The long-term outcome of ulcerative colitis treated with 6-mercaptopurine. Am J Gastroenterol 1996; 91 : $1711-1714$

[427] Fraser AG, Orchard TR, Jewell DP. The efficacy of azathioprine for the treatment of inflammatory bowel disease: a 30 year review. Gut 2002; 50: $485-489$

[428] Ardizzone S, Molteni P, Imbesi V et al. Azathioprine in steroid-resistant and steroid-dependent ulcerative colitis. J Clin Gastroenterol 1997; 25 $330-333$

[429] Lobo AJ, Foster PN, Burke DA et al. The role of azathioprine in the management of ulcerative colitis. Dis Colon Rectum 1990; 33: 374 377

[430] Khan ZH, Mayberry JF, Spiers N et al. Retrospective case series analysis of patients with inflammatory bowel disease on azathioprine. A district general hospital experience. Digestion 2000; 62: 249-254

[431] Adler DJ, Korelitz BI. The therapeutic efficacy of 6-mercaptopurine in refractory ulcerative colitis. Am J Gastroenterol 1990; 85: 717-722

[432] Moreno-Rincon E, Benitez JM, Serrano-Ruiz F] et al. Prognosis of Patients with Ulcerative Colitis in Sustained Remission After Thiopurines Withdrawal. Inflamm Bowel Dis 2015; 21: 1564 - 1571

[433] Fernandez-Banares F, Bertran X, Esteve-Comas M et al. Azathioprine is useful in maintaining long-term remission induced by intravenous cyclosporine in steroid-refractory severe ulcerative colitis. Am J Gastroenterol 1996; 91: 2498-2499

[434] Ferrante $M$, Vermeire $S$, Fidder $\mathrm{H}$ et al. Long-term outcome after infliximab for refractory ulcerative colitis. J Crohns Colitis 2008; 2: 219 225

[435] Feagan BG, Sandborn WJ, Lazar A et al. Adalimumab therapy is associated with reduced risk of hospitalization in patients with ulcerative colitis. Gastroenterology 2014; 146: 110 - 118 e3

[436] Colombel JF, Sandborn W], Ghosh S et al. Four-year maintenance treatment with adalimumab in patients with moderately to severely active ulcerative colitis: Data from ULTRA 1, 2, and 3. Am J Gastroenterol 2014; 109: 1771 - 1780

[437] Loftus EV Jr, Colombel JF, Feagan BG et al. Long-term Efficacy of Vedolizumab for Ulcerative Colitis. J Crohns Colitis 2017; 11: 400-411

[438] Feagan BG, Rubin DT, Danese S et al. Efficacy of Vedolizumab Induction and Maintenance Therapy in Patients With Ulcerative Colitis, Regardless of Prior Exposure to Tumor Necrosis Factor Antagonists. Clin Gastroenterol Hepatol 2017; 15: 229-239 e5

[439] Noman M, Ferrante M, Bisschops R et al. Vedolizumab Induces Longterm Mucosal Healing in Patients With Crohn's Disease and Ulcerative Colitis. J Crohns Colitis 2017; 11: 1085-1089

[440] Stallmach A, Langbein C, Atreya R et al. Vedolizumab provides clinical benefit over 1 year in patients with active inflammatory bowel disease - a prospective multicenter observational study. Aliment Pharmacol Ther 2016; 44: 1199-1212

[441] Amiot A, Serrero M, Peyrin-Biroulet $L$ et al. One-year effectiveness and safety of vedolizumab therapy for inflammatory bowel disease: a prospective multicentre cohort study. Aliment Pharmacol Ther 2017; 46: $310-321$

[442] Bickston S], Behm BW, Tsoulis DJ et al. Vedolizumab for induction and maintenance of remission in ulcerative colitis. Cochrane Database Syst Rev 2014; 8: CD007571

[443] Ungar B, Kopylov U, Engel T et al. Addition of an immunomodulator can reverse antibody formation and loss of response in patients treated with adalimumab. Aliment Pharmacol Ther 2017; 45: 276-282

[444] Matsumoto T, Motoya S, Watanabe K et al. Adalimumab Monotherapy and a Combination with Azathioprine for Crohn's Disease: A Prospective, Randomized Trial. J Crohns Colitis 2016; 10: 1259-1266 
[445] Oren R, Arber N, Odes S et al. Methotrexate in chronic active ulcerative colitis: a double-blind, randomized, Israeli multicenter trial. Gastroenterology 1996; 110: 1416-1421

[446] Wahed M, Louis-Auguste JR, Baxter LM et al. Efficacy of methotrexate in Crohn's disease and ulcerative colitis patients unresponsive or intolerant to azathioprine /mercaptopurine. Aliment Pharmacol Ther 2009; 30: $614-620$

[447] Wang Y, MacDonald JK, Vandermeer B et al. Methotrexate for maintenance of remission in ulcerative colitis. Cochrane Database Syst Rev 2015; 11: CD007560

[448] Torres ], Boyapati RK, Kennedy NA et al. Systematic Review of Effects of Withdrawal of Immunomodulators or Biologic Agents From Patients With Inflammatory Bowel Disease. Gastroenterology 2015; 149: $1716-1730$

[449] Marits P, Landucci L, Sundin U et al. Trough s-infliximab and antibodies towards infliximab in a cohort of 79 IBD patients with maintenance infliximab treatment. J Crohns Colitis 2014; 8: 881-889

[450] Mazor Y, Almog R, Kopylov U et al. Adalimumab drug and antibody levels as predictors of clinical and laboratory response in patients with Crohn's disease. Aliment Pharmacol Ther 2014; 40: 620-628

[451] Cornillie F, Hanauer SB, Diamond RH et al. Postinduction serum infliximab trough level and decrease of $C$-reactive protein level are associated with durable sustained response to infliximab: a retrospective analysis of the ACCENT I trial. Gut 2014; 63: 1721 - 1727

[452] Pouw MF, Krieckaert CL, Nurmohamed MT et al. Key findings towards optimising adalimumab treatment: the concentration-effect curve. Ann Rheum Dis 2015; 74: 513-518

[453] Seow CH, Newman A, Irwin SP et al. Trough serum infliximab: a predictive factor of clinical outcome for infliximab treatment in acute ulcerative colitis. Gut 2010; 59: 49-54

[454] Moore C, Corbett G, Moss AC. Systematic Review and Meta-Analysis: Serum Infliximab Levels During Maintenance Therapy and Outcomes in Inflammatory Bowel Disease. J Crohns Colitis 2016; 10: 619-625

[455] Roblin X, Marotte H, Rinaudo M et al. Association between pharmacokinetics of adalimumab and mucosal healing in patients with inflammatory bowel diseases. Clin Gastroenterol Hepatol 2014; 12: 80 - 84 e2

[456] Harzallah I, Rigaill ], Williet $\mathrm{N}$ et al. Golimumab pharmacokinetics in ulcerative colitis: a literature review. Therap Adv Gastroenterol 2017; 10: $89-100$

[457] Afif W, Loftus EV Jr, Faubion WA et al. Clinical utility of measuring infliximab and human anti-chimeric antibody concentrations in patients with inflammatory bowel disease. Am J Gastroenterol 2010; 105: $1133-1139$

[458] Frederiksen MT, Ainsworth MA, Brynskov J et al. Antibodies against infliximab are associated with de novo development of antibodies to adalimumab and therapeutic failure in infliximab-to-adalimumab switchers with IBD. Inflamm Bowel Dis 2014; 20: 1714-1721

[459] O'Meara S, Nanda KS, Moss AC. Antibodies to infliximab and risk of infusion reactions in patients with inflammatory bowel disease: a systematic review and meta-analysis. Inflamm Bowel Dis 2014; 20: 1-6

[460] Ungar B, Chowers Y, Yavzori M et al. The temporal evolution of antidrug antibodies in patients with inflammatory bowel disease treated with infliximab. Gut 2014; 63: 1258-1264

[461] Steenholdt C, Al-khalaf M, Brynskov J et al. Clinical implications of variations in anti-infliximab antibody levels in patients with inflammatory bowel disease. Inflamm Bowel Dis 2012; 18: 2209-2217

[462] Yanai H, Lichtenstein L, Assa A et al. Levels of drug and antidrug antibodies are associated with outcome of interventions after loss of response to infliximab or adalimumab. Clin Gastroenterol Hepatol 2015; 13: $522-530$ e2
[463] Yacoub W, Williet N, Pouillon L et al. Early vedolizumab trough levels predict mucosal healing in inflammatory bowel disease: a multicentre prospective observational study. Aliment Pharmacol Ther 2018; 47: 906-912

[464] Schulze H, Esters P, Hartmann F et al. A prospective cohort study to assess the relevance of vedolizumab drug level monitoring in IBD patients. Scand J Gastroenterol 2018; 53: 670-676

[465] Dreesen E, Verstockt B, Bian S et al. Evidence to Support Monitoring of Vedolizumab Trough Concentrations in Patients With Inflammatory Bowel Diseases. Clin Gastroenterol Hepatol 2018. S15423565(18)30402-6

[466] Baumgart DC, Pintoffl JP, Sturm A et al. Tacrolimus is safe and effective in patients with severe steroid-refractory or steroid-dependent inflammatory bowel disease-a long-term follow-up. Am J Gastroenterol 2006; 101: $1048-1056$

[467] Luthra P, Peyrin-Biroulet L, Ford AC. Systematic review and meta-analysis: opportunistic infections and malignancies during treatment with anti-integrin antibodies in inflammatory bowel disease. Aliment Pharmacol Ther 2015; 41: 1227 - 1236

[468] Brassard P, Bitton A, Suissa A et al. Oral corticosteroids and the risk of serious infections in patients with elderly-onset inflammatory bowel diseases. Am J Gastroenterol 2014; 109: 1795-1802; quiz 1803

[469] Stuck AE, Minder CE, Frey FJ. Risk of infectious complications in patients taking glucocorticosteroids. Rev Infect Dis 1989; 11: 954-963

[470] Dixon WG, Kezouh A, Bernatsky S et al. The influence of systemic glucocorticoid therapy upon the risk of non-serious infection in older patients with rheumatoid arthritis: a nested case-control study. Ann Rheum Dis 2011; 70: 956-960

[471] Jick SS, Lieberman ES, Rahman MU et al. Glucocorticoid use, other associated factors, and the risk of tuberculosis. Arthritis Rheum 2006; 55: $19-26$

[472] Ananthakrishnan AN, McGinley EL. Infection-related hospitalizations are associated with increased mortality in patients with inflammatory bowel diseases. J Crohns Colitis 2013; 7: 107-112

[473] Ha CY. Risks of Infection among the Older Inflammatory Bowel Disease Patients. Curr Treat Options Gastroenterol 2014; 12: 283 - 291

[474] Castle SC. Clinical relevance of age-related immune dysfunction. Clin Infect Dis 2000; 31: 578-585

[475] Strangfeld A, Eveslage M, Schneider M et al. Treatment benefit or survival of the fittest: what drives the time-dependent decrease in serious infection rates under TNF inhibition and what does this imply for the individual patient? Ann Rheum Dis 2011; 70: 1914-1920

[476] Ananthakrishnan AN, Cagan A, Cai T et al. Diabetes and the risk of infections with immunomodulator therapy in inflammatory bowel diseases. Aliment Pharmacol Ther 2015; 41: 1141-1148

[477] Blum HE, Berg T, Tillmann HL et al. Procedures for problematic situations with hepatitis B. Z Gastroenterol 2004; 42: 692-697

[478] Domm S, Cinatl ], Mrowietz U. The impact of treatment with tumour necrosis factor-alpha antagonists on the course of chronic viral infections: a review of the literature. $\mathrm{Br}$ J Dermatol 2008; 159: 1217-1228

[479] Mindikoglu AL, Regev A, Schiff ER. Hepatitis B virus reactivation after cytotoxic chemotherapy: the disease and its prevention. Clin Gastroenterol Hepatol 2006; 4: 1076-1081

[480] Esteve M, Saro C, Gonzalez-Huix F et al. Chronic hepatitis B reactivation following infliximab therapy in Crohn's disease patients: need for primary prophylaxis. Gut 2004; 53: 1363 - 1365

[481] Lau GK. Hepatitis B reactivation after chemotherapy: two decades of clinical research. Hepatol Int 2008; 2: 152-162

[482] Cornberg M, Protzer U, Petersen J et al. Prophylaxis, diagnosis and therapy of hepatitis B virus infection - the German guideline. Z Gastroenterol 2011; 49: 871 - 930 
[483] Diel R, Forssbohm M, Loytved G et al. Empfehlungen für die Umgebungsuntersuchungen bei Tuberkulose. Pneumologie 2007; 61: 440 455

[484] Schoepfer AM, Flogerzi B, Fallegger S et al. Comparison of interferongamma release assay versus tuberculin skin test for tuberculosis screening in inflammatory bowel disease. Am J Gastroenterol 2008; 103: $2799-2806$

[485] Hatzara C, Hadziyannis E, Kandili A et al. Frequent conversion of tuberculosis screening tests during anti-tumour necrosis factor therapy in patients with rheumatic diseases. Ann Rheum Dis 2015; 74: 1848 1853

[486] Thorley-Lawson DA. EBV the prototypical human tumor virus-just how bad is it? J Allergy Clin Immunol 2005; 116: 251 -261; quiz 262

[487] Thompson MP, Kurzrock R. Epstein-Barr virus and cancer. Clin Cancer Res 2004; 10: $803-821$

[488] Funch DP, Walker AM, Schneider G et al. Ganciclovir and acyclovir reduce the risk of post-transplant lymphoproliferative disorder in renal transplant recipients. Am J Transplant 2005; 5: 2894-2900

[489] Reddy N, Rezvani K, Barrett AJ et al. Strategies to prevent EBV reactivation and posttransplant lymphoproliferative disorders (PTLD) after allogeneic stem cell transplantation in high-risk patients. Biol Blood Marrow Transplant 2011; 17: 591 - 597

[490] Stobaugh DJ, Deepak P, Ehrenpreis ED. Hospitalizations for vaccine preventable pneumonias in patients with inflammatory bowel disease: a 6-year analysis of the Nationwide Inpatient Sample. Clin Exp Gastroenterol 2013; 6: 43-49

[491] Teich N, Klugmann T, Tiedemann A et al. Vaccination coverage in immunosuppressed patients: results of a regional health services research study. Dtsch Arztebl Int 2011; 108: 105-111

[492] Borte S, Liebert UG, Borte M et al. Efficacy of measles, mumps and rubella revaccination in children with juvenile idiopathic arthritis treated with methotrexate and etanercept. Rheumatology (Oxford) 2009; 48: $144-148$

[493] Nguyen DL, Nguyen ET, Bechtold ML. Effect of Immunosuppressive Therapies for the Treatment of Inflammatory Bowel Disease on Response to Routine Vaccinations: A Meta-Analysis. Dig Dis Sci 2015; 60: $2446-2453$

[494] Poppers DM, Scherl EJ. Prophylaxis against Pneumocystis pneumonia in patients with inflammatory bowel disease: toward a standard of care. Inflamm Bowel Dis 2008; 14: 106-113

[495] Cotter TG, Gathaiya N, Catania J et al. Low Risk of Pneumonia From Pneumocystis jirovecii Infection in Patients With Inflammatory Bowel Disease Receiving Immune Suppression. Clin Gastroenterol Hepatol 2017; 15: $850-856$

[496] Long MD, Farraye FA, Okafor PN et al. Increased risk of pneumocystis jiroveci pneumonia among patients with inflammatory bowel disease. Inflamm Bowel Dis 2013; 19: 1018-1024

[497] Stern A, Green H, Paul M et al. Prophylaxis for Pneumocystis pneumonia (PCP) in non-HIV immunocompromised patients. Cochrane Database Syst Rev 2014; 1: CD005590

[498] Messiaen PE, Cuyx S, Dejagere T et al. The role of CD4 cell count as discriminatory measure to guide chemoprophylaxis against Pneumocystis jirovecii pneumonia in human immunodeficiency virus-negative immunocompromised patients: A systematic review. Transpl Infect Dis 2017; 19

[499] Ioannidis JP, Cappelleri JC, Skolnik PR et al. A meta-analysis of the relative efficacy and toxicity of Pneumocystis carinii prophylactic regimens. Arch Intern Med 1996; 156: 177-188

[500] Stallmach A, Carstens O. Role of infections in the manifestation or reactivation of inflammatory bowel diseases. Inflamm Bowel Dis 2002; 8: $213-218$
[501] Ananthakrishnan AN, McGinley EL, Binion DG. Excess hospitalisation burden associated with Clostridium difficile in patients with inflammatory bowel disease. Gut 2008; 57: 205-210

[502] Das R, Feuerstadt P, Brandt L]. Glucocorticoids are associated with increased risk of short-term mortality in hospitalized patients with clostridium difficile-associated disease. Am J Gastroenterol 2010; 105: $2040-2049$

[503] Schneeweiss S, Korzenik J, Solomon DH et al. Infliximab and other immunomodulating drugs in patients with inflammatory bowel disease and the risk of serious bacterial infections. Aliment Pharmacol Ther 2009; 30: $253-264$

[504] Singh H, Nugent Z, Yu BN et al. Higher Incidence of Clostridium difficile Infection Among Individuals With Inflammatory Bowel Disease. Gastroenterology 2017; 153: 430-438 e2

[505] Dallal RM, Harbrecht BG, Boujoukas A] et al. Fulminant Clostridium difficile: an underappreciated and increasing cause of death and complications. Ann Surg 2002; 235: $363-372$

[506] Asha NJ, Tompkins D, Wilcox MH. Comparative analysis of prevalence, risk factors, and molecular epidemiology of antibiotic-associated diarrhea due to Clostridium difficile, Clostridium perfringens, and Staphylococcus aureus. J Clin Microbiol 2006; 44: 2785-2791

[507] Crobach M], Planche T, Eckert C et al. European Society of Clinical Microbiology and Infectious Diseases: update of the diagnostic guidance document for Clostridium difficile infection. Clin Microbiol Infect 2016; 22 (Suppl. 4): S63-S81

[508] von Müller L, Halfmann A, Herrmann M. Current data and trends on the development of antibiotic resistance of Clostridium difficile. Bundesgesundheitsblatt Gesundheitsforschung Gesundheitsschutz 2012; 55 : $1410-1417$

[509] Erb S, Frei R, Stranden AM et al. Low sensitivity of fecal toxin A/B enzyme immunoassay for diagnosis of Clostridium difficile infection in immunocompromised patients. Clin Microbiol Infect 2015; 21: 998 e9-998 e15

[510] Vonberg RP, Kuijper EJ, Wilcox MH et al. Infection control measures to limit the spread of Clostridium difficile. Clin Microbiol Infect 2008; 14 (Suppl. 5): 2-20

[511] Fekety R, Silva J, Kauffman C et al. Treatment of antibiotic-associated Clostridium difficile colitis with oral vancomycin: comparison of two dosage regimens. Am J Med 1989; 86: 15-19

[512] Gerding DN, Muto CA, Owens RC et al. Treatment of Clostridium difficile infection. Clin Infect Dis 2008; 46 (Suppl. 1): S32 - S42

[513] Kuijper EJ, Wilcox MH. Decreased effectiveness of metronidazole for the treatment of Clostridium difficile infection? Clin Infect Dis 2008; 47: $63-65$

[514] Horton HA, Dezfoli S, Berel D et al. Antibiotics for Treatment of Clostridium difficile Infection in Hospitalized Patients with Inflammatory Bowel Disease. Antimicrob Agents Chemother 2014; 58: 5054 - 5059

[515] Louie T], Miller MA, Mullane KM et al. Fidaxomicin versus vancomycin for Clostridium difficile infection. N Engl J Med 2011; 364: 422-431

[516] Crook DW, Walker AS, Kean Y et al. Fidaxomicin versus vancomycin for Clostridium difficile infection: meta-analysis of pivotal randomized controlled trials. Clin Infect Dis 2012; 55 (Suppl. 2): S93 - S103

[517] Hoegenauer C, Mahida Y, Stallmach A. Open-label study to evaluate the pharmacokinetics of fidaxomicin in inflammatory bowel disease patients with Clostridium difficile infection (the PROFILE study): pharmacokinetics analysis. Journal of Crohn's and Colitis 2017 (Suppl. 1): S276

[518] Nelson RL, Suda KJ, Evans CT. Antibiotic treatment for Clostridium difficile-associated diarrhoea in adults. Cochrane Database Syst Rev 2017; 3: CD004610 
[519] Shen NT, Maw A, Tmanova LL et al. Timely Use of Probiotics in Hospitalized Adults Prevents Clostridium difficile Infection: A Systematic Review With Meta-Regression Analysis. Gastroenterology 2017; 152: 1889-1900 e9

[520] McFarland LV, Elmer GW, Surawicz CM. Breaking the cycle: treatment strategies for 163 cases of recurrent Clostridium difficile disease. Am J Gastroenterol 2002; 97: 1769-1775

[521] Eiseman B, Silen W, Bascom GS et al. Fecal enema as an adjunct in the treatment of pseudomembranous enterocolitis. Surgery 1958; 44 : $854-859$

[522] van Nood E, Vrieze A, Nieuwdorp M et al. Duodenal infusion of donor feces for recurrent Clostridium difficile. N Engl J Med 2013; 368: 407 415

[523] Fischer M, Kao D, Kelly C et al. Fecal Microbiota Transplantation is Safe and Efficacious for Recurrent or Refractory Clostridium difficile Infection in Patients with Inflammatory Bowel Disease. Inflamm Bowel Dis 2016; 22: 2402-2409

[524] Khoruts A, Rank KM, Newman KM et al. Inflammatory Bowel Disease Affects the Outcome of Fecal Microbiota Transplantation for Recurrent Clostridium difficile Infection. Clin Gastroenterol Hepatol 2016; 14: $1433-1438$

[525] Teich N, Weber M, Stallmach A. First Occurrence of Severe Extraintestinal Manifestations of Crohn's Disease Following Faecal Microbiota Transplantation. J Crohns Colitis 2016; 10: 1254-1255

[526] Beaugerie L, Brousse N, Bouvier AM et al. Lymphoproliferative disorders in patients receiving thiopurines for inflammatory bowel disease: a prospective observational cohort study. Lancet 2009; 374: 1617 1625

[527] Posthuma EF, Westendorp RG, van der Sluys Veer A et al. Fatal infectious mononucleosis: a severe complication in the treatment of Crohn's disease with azathioprine. Gut 1995; 36: 311-313

[528] Garrido Serrano A, Perez Martin F, Guerrero Igea FJ et al. Fatal infectious mononucleosis during azathioprine treatment in Crohn's disease. Gastroenterol Hepatol 2000; 23: 7-8

[529] N'Guyen Y, Andreoletti L, Patey M et al. Fatal Epstein-Barr virus primo infection in a 25-year-old man treated with azathioprine for Crohn's disease. J Clin Microbiol 2009; 47: 1252-1254

[530] Nissen LH, Nagtegaal ID, de Jong D] et al. Epstein-Barr virus in inflammatory bowel disease: the spectrum of intestinal lymphoproliferative disorders. J Crohns Colitis 2015; 9: 398-403

[531] Fries W, Cottone M, Cascio A. Systematic review: macrophage activation syndrome in inflammatory bowel disease. Aliment Pharmacol Ther 2013; 37: 1033-1045

[532] Virdis F, Tacci S, Messina F et al. Hemophagocytic lymphohistiocytosis caused by primary Epstein-Barr virus in patient with Crohn's disease. World J Gastrointest Surg 2013; 5: 306 -308

[533] Klein S, Schmidt C, La Rosee P et al. Fulminant gastrointestinal bleeding caused by EBV-triggered hemophagocytic lymphohistiocytosis: report of a case. Z Gastroenterol 2014; 52: 354-359

[534] Brito-Zeron P, Bosch X, Perez-de-Lis M et al. Infection is the major trigger of hemophagocytic syndrome in adult patients treated with biological therapies. Semin Arthritis Rheum 2016; 45: 391 - 399

[535] Cottone M, Pietrosi G, Martorana G et al. Prevalence of cytomegalovirus infection in severe refractory ulcerative and Crohn's colitis. Am J Gastroenterol 2001; 96: 773-775

[536] Kambham N, Vij R, Cartwright CA et al. Cytomegalovirus infection in steroid-refractory ulcerative colitis: a case-control study. Am J Surg Pathol 2004; 28: 365 - 373

[537] Tandon P, James P, Cordeiro E et al. Diagnostic Accuracy of BloodBased Tests and Histopathology for Cytomegalovirus Reactivation in Inflammatory Bowel Disease: A Systematic Review and Meta-Analysis. Inflamm Bowel Dis 2017; 23: 551-560
[538] Roblin X, Pillet S, Oussalah A et al. Cytomegalovirus load in inflamed intestinal tissue is predictive of resistance to immunosuppressive therapy in ulcerative colitis. Am J Gastroenterol 2011; 106: 2001 2008

[539] Zidar N, Ferkolj I, Tepes K et al. Diagnosing cytomegalovirus in patients with inflammatory bowel disease-by immunohistochemistry or polymerase chain reaction? Virchows Arch 2015; 466: 533-539

[540] Thorn M, Rorsman F, Ronnblom A et al. Active cytomegalovirus infection diagnosed by real-time PCR in patients with inflammatory bowel disease: a prospective, controlled observational study (.). Scand J Gastroenterol 2016; 51: 1075-1080

[541] Romkens TE, Bulte G], Nissen LH et al. Cytomegalovirus in inflammatory bowel disease: A systematic review. World J Gastroenterol 2016; 22: $1321-1330$

[542] Shukla T, Singh S, Loftus EV Jr et al. Antiviral Therapy in Steroid-refractory Ulcerative Colitis with Cytomegalovirus: Systematic Review and Meta-analysis. Inflamm Bowel Dis 2015; 21: 2718-2725

[543] Kopylov U, Eliakim-Raz N, Szilagy A et al. Antiviral therapy in cytomegalovirus-positive ulcerative colitis: a systematic review and metaanalysis. World J Gastroenterol 2014; 20: 2695-2703

[544] Beswick L, Ye B, van Langenberg DR. Toward an Algorithm for the Diagnosis and Management of CMV in Patients with Colitis. Inflamm Bowel Dis 2016; 22: 2966-2976

[545] Kopylov U, Papamichael K, Katsanos K et al. Impact of Infliximab and Cyclosporine on the Risk of Colectomy in Hospitalized Patients with Ulcerative Colitis Complicated by Cytomegalovirus-A Multicenter Retrospective Study. Inflamm Bowel Dis 2017; 23: 1605-1613

[546] Maconi G, Colombo E, Zerbi P et al. Prevalence, detection rate and outcome of cytomegalovirus infection in ulcerative colitis patients requiring colonic resection. Dig Liver Dis 2005; 37: 418-423

[547] Kotton CN, Kumar D, Caliendo AM et al. Updated international consensus guidelines on the management of cytomegalovirus in solid-organ transplantation. Transplantation 2013; 96: 333-360

[548] Cullen G, Baden RP, Cheifetz AS. Varicella zoster virus infection in inflammatory bowel disease. Inflamm Bowel Dis 2012; 18: 2392 - 2403

[549] Cote-Daigneault J, Peerani F, MacMahon E et al. Management and Prevention of Herpes Zoster in the Immunocompromised Inflammatory Bowel Disease Patient: A Clinical Quandary. Inflamm Bowel Dis 2016; 22: $2538-2547$

[550] Robert Koch-Institut. Tuberkulose. RKI-Ratgeber für Ärzte. 2017 http://www.rki.de/DE/Content/Infekt/EpidBull/Merkblaetter/ Ratgeber_Tuberkulose.htm

[551] Lewinsohn DM, Leonard MK, LoBue PA et al. Official American Thoracic Society/Infectious Diseases Society of America/Centers for Disease Control and Prevention Clinical Practice Guidelines: Diagnosis of Tuberculosis in Adults and Children. Clin Infect Dis 2017; 64: 111 - 115

[552] Julsgaard M, Christensen LA, Gibson PR et al. Concentrations of Adalimumab and Infliximab in Mothers and Newborns, and Effects on Infection. Gastroenterology 2016; 151: 110-119

[553] Lichtenstein GR, Cohen R, Yamashita B et al. Quality of life after proctocolectomy with ileoanal anastomosis for patients with ulcerative colitis. J Clin Gastroenterol 2006; 40: 669-677

[554] Heuschen UA, Heuschen G, Rudek B et al. Long-term quality of life after continence-preserving proctocolectomy for ulcerative colitis and familial adenomatous polyposis. Chirurg 1998; 69: 1052-1058

[555] Mark-Christensen A, Erichsen R, Brandsborg S et al. Pouch Failures Following lleal Pouch-anal Anastomosis for Ulcerative Colitis. Colorectal Dis 2018; 20: 44-52

[556] Lovegrove RE, Tilney HS, Remzi FH et al. To divert or not to divert: A retrospective analysis of variables that influence ileostomy omission in ileal pouch surgery. Arch Surg 2011; 146: $82-88$ 
[557] Mennigen R, Sewald W, Senninger N et al. Morbidity of loop ileostomy closure after restorative proctocolectomy for ulcerative colitis and familial adenomatous polyposis: a systematic review. J Gastrointest Surg 2014; 18: $2192-2200$

[558] Lovegrove RE, Heriot AG, Constantinides V et al. Meta-analysis of short-term and long-term outcomes of J, W and S ileal reservoirs for restorative proctocolectomy. Colorectal Dis 2007; 9: 310-320

[559] McCormick PH, Guest GD, Clark A] et al. The ideal ileal-pouch design: a long-term randomized control trial of J- vs W-pouch construction. Dis Colon Rectum 2012; 55: 1251 - 1257

[560] Oresland T, Fasth S, Nordgren S et al. A prospective randomized comparison of two different pelvic pouch designs. Scand J Gastroenterol 1990; 25: $986-996$

[561] Pal S, Sahni P, Pande GK et al. Outcome following emergency surgery for refractory severe ulcerative colitis in a tertiary care centre in India. BMC Gastroenterol 2005; 5: 39

[562] Heppell ], Farkouh E, Dube S et al. Toxic megacolon. An analysis of 70 cases. Dis Colon Rectum 1986; 29: $789-792$

[563] Fowkes L, Krishna K, Menon A et al. Laparoscopic emergency and elective surgery for ulcerative colitis. Colorectal Dis 2008; 10: 373 378

[564] Ziv Y, Fazio VW, Church JM et al. Safety of urgent restorative proctocolectomy with ileal pouch-anal anastomosis for fulminant colitis. Dis Colon Rectum 1995; 38: 345-349

[565] Heyvaert G, Penninckx F, Filez L et al. Restorative proctocolectomy in elective and emergency cases of ulcerative colitis. Int J Colorectal Dis 1994; $9: 73-76$

[566] Fazio VW, Ziv Y, Church JM et al. Ileal pouch-anal anastomoses complications and function in 1005 patients. Ann Surg 1995; 222: 120 127

[567] Sheth SG, LaMont JT. Toxic megacolon. Lancet 1998; 351: 509-513

[568] Croft A, Walsh A, Doecke J et al. Outcomes of salvage therapy for steroid-refractory acute severe ulcerative colitis: ciclosporin vs. infliximab. Aliment Pharmacol Ther 2013; 38: 294-302

[569] Turner D, Hyams J, Markowitz J et al. Appraisal of the pediatric ulcerative colitis activity index (PUCAI). Inflamm Bowel Dis 2009; 15: 1218 1223

[570] Turner D, Walsh CM, Benchimol El et al. Severe paediatric ulcerative colitis: incidence, outcomes and optimal timing for second-line therapy. Gut 2008; 57: $331-338$

[571] Kimura H, Kunisaki R, Tatsumi K et al. Prolonged Medical Therapy Increases the Risk of Surgical Complications in Patients with Severe Ulcerative Colitis. Dig Surg 2016; 33: 182 - 189

[572] Neumann PA, Mennigen RB, Senninger $N$ et al. Timing of restorative proctocolectomy in patients with medically refractory ulcerative colitis: the patient's point of view. Dis Colon Rectum 2012; 55: 756 - 761

[573] Feuerstein JD, Akbari M, Tapper EB et al. Systematic review and metaanalysis of third-line salvage therapy with infliximab or cyclosporine in severe ulcerative colitis. Ann Gastroenterol 2016; 29: 341 - 347

[574] van der Valk ME, Mangen MJ, Severs M et al. Comparison of Costs and Quality of Life in Ulcerative Colitis Patients with an Ileal Pouch-Anal Anastomosis, lleostomy and Anti-TNFalpha Therapy. J Crohns Colitis 2015; 9: 1016-1023

[575] Heuschkel R, Salvestrini C, Beattie RM et al. Guidelines for the management of growth failure in childhood inflammatory bowel disease. Inflamm Bowel Dis 2008; 14: 839-849

[576] Nicholls RJ, Holt SD, Lubowski DZ. Restorative proctocolectomy with ileal reservoir. Comparison of two-stage vs. three-stage procedures and analysis of factors that might affect outcome. Dis Colon Rectum 1989; 32 : $323-326$
[577] Selvasekar CR, Cima RR, Larson DW et al. Effect of infliximab on shortterm complications in patients undergoing operation for chronic ulcerative colitis. J Am Coll Surg 2007; 204: 956-962; discussion 962963

[578] Stewart D, Chao A, Kodner I et al. Subtotal colectomy for toxic and fulminant colitis in the era of immunosuppressive therapy. Colorectal Dis 2009; 11: $184-190$

[579] Shen B. Impact of preoperative infliximab use on postoperative infectious complications in ulcerative colitis: the price we have to pay? Inflamm Bowel Dis 2008; 14: 1019-1021

[580] Norgard BM, Nielsen J, Qvist N et al. Pre-operative use of antiTNF-alpha agents and the risk of post-operative complications in patients with ulcerative colitis - a nationwide cohort study. Aliment Pharmacol Ther 2012; 35: 1301 - 1309

[581] Mor I], Vogel JD, da Luz Moreira A et al. Infliximab in ulcerative colitis is associated with an increased risk of postoperative complications after restorative proctocolectomy. Dis Colon Rectum 2008; 51: 1202 1207; discussion 1207-1210

[582] Yang Z, Wu Q, Wang F et al. Meta-analysis: effect of preoperative infliximab use on early postoperative complications in patients with ulcerative colitis undergoing abdominal surgery. Aliment Pharmacol Ther 2012; 36: $922-928$

[583] Selvaggi F, Pellino G, Canonico S et al. Effect of preoperative biologic drugs on complications and function after restorative proctocolectomy with primary ileal pouch formation: systematic review and metaanalysis. Inflamm Bowel Dis 2015; 21: 79-92

[584] Kulaylat AS, Kulaylat AN, Schaefer EW et al. Association of Preoperative Anti-Tumor Necrosis Factor Therapy With Adverse Postoperative Outcomes in Patients Undergoing Abdominal Surgery for Ulcerative Colitis. JAMA Surg 2017; 152: e171538

[585] Lau C, Dubinsky M, Melmed G et al. The impact of preoperative serum anti-TNFalpha therapy levels on early postoperative outcomes in inflammatory bowel disease surgery. Ann Surg 2015; 261: 487-496

[586] Hait EJ, Bousvaros A, Schuman M et al. Pouch outcomes among children with ulcerative colitis treated with calcineurin inhibitors before ileal pouch anal anastomosis surgery. J Pediatr Surg 2007; 42: 31 - 34; discussion 34-35

[587] Gu J, Stocchi L, Remzi F et al. Intraperitoneal or subcutaneous: does location of the (colo)rectal stump influence outcomes after laparoscopic total abdominal colectomy for ulcerative colitis? Dis Colon Rectum 2013; 56: 615-621

[588] Trickett JP, Tilney HS, Gudgeon AM et al. Management of the rectal stump after emergency sub-total colectomy: which surgical option is associated with the lowest morbidity? Colorectal Dis 2005; 7: 519522

[589] Karch LA, Bauer J], Gorfine SR et al. Subtotal colectomy with Hartmann's pouch for inflammatory bowel disease. Dis Colon Rectum 1995; 38: $635-639$

[590] Bartels SA, Gardenbroek T], Aarts M et al. Short-term morbidity and quality of life from a randomized clinical trial of close rectal dissection and total mesorectal excision in ileal pouch-anal anastomosis. $\mathrm{Br}$ J Surg 2015; 102: $281-287$

[591] Hicks CW, Hodin RA, Savitt L et al. Does intramesorectal excision for ulcerative colitis impact bowel and sexual function when compared with total mesorectal excision? Am J Surg 2014; 208: 499-504 e4

[592] Rink AD, Radinski I, Vestweber KH. Does mesorectal preservation protect the ileoanal anastomosis after restorative proctocolectomy? J Gastrointest Surg 2009; 13: 120 - 128

[593] Lovegrove RE, Constantinides VA, Heriot AG et al. A comparison of hand-sewn versus stapled ileal pouch anal anastomosis (IPAA) following proctocolectomy: a meta-analysis of 4183 patients. Ann Surg 2006; $244: 18-26$ 
[594] Schluender SJ, Mei L, Yang H et al. Can a meta-analysis answer the question: is mucosectomy and handsewn or double-stapled anastomosis better in ileal pouch-anal anastomosis? Am Surg 2006; 72: 912 916

[595] Ganschow P, Treiber I, Hinz U et al. Residual rectal mucosa after stapled vs. handsewn ileal J-pouch-anal anastomosis in patients with familial adenomatous polyposis coli (FAP) -a critical issue. Langenbecks Arch Surg 2015; 400: 213-219

[596] Ota H, Yamazaki K, Endoh W et al. Adenocarcinoma arising below an ileoanal anastomosis after restorative proctocolectomy for ulcerative colitis: report of a case. Surg Today 2007; 37: 596-599

[597] Alessandroni L, Kohn A, Capaldi M et al. Adenocarcinoma below stapled ileoanal anastomosis after restorative proctocolectomy for ulcerative colitis. Updates Surg 2012; 64: 149-152

[598] Al-Sukhni W, McLeod RS, MacRae H et al. Oncologic outcome in patients with ulcerative colitis associated with dyplasia or cancer who underwent stapled or handsewn ileal pouch-anal anastomosis. Dis Colon Rectum 2010; 53: 1495-1500

[599] Oresland T, Bemelman WA, Sampietro GM et al. European evidence based consensus on surgery for ulcerative colitis. J Crohns Colitis 2015; 9: $4-25$

[600] Tekkis PP, Fazio VW, Lavery IC et al. Evaluation of the learning curve in ileal pouch-anal anastomosis surgery. Ann Surg 2005; 241: $262-268$

[601] Parc Y, Reboul-Marty J, Lefevre JH et al. Restorative Proctocolectomy and Ileal Pouch-anal Anastomosis. Ann Surg 2015; 262: 849-853; discussion 853-854

[602] Burns EM, Bottle A, Aylin P et al. Volume analysis of outcome following restorative proctocolectomy. $\mathrm{Br}$ J Surg 2011; 98: 408-417

[603] Raval M], Schnitzler M, O’Connor Bl et al. Improved outcome due to increased experience and individualized management of leaks after ileal pouch-anal anastomosis. Ann Surg 2007; 246: 763 - 770

[604] Borjesson L, Willen R, Haboubi N et al. The risk of dysplasia and cancer in the ileal pouch mucosa after restorative proctocolectomy for ulcerative proctocolitis is low: a long-term term follow-up study. Colorectal Dis 2004; 6: $494-498$

[605] Pachler FR, Brandsborg SB, Laurberg S. Paradoxical Impact of Ileal Pouch-Anal Anastomosis on Male and Female Fertility in Patients With Ulcerative Colitis. Dis Colon Rectum 2017; 60: 603-607

[606] Uzzan M, Cosnes ], Amiot A et al. Long-term Follow-up After lleorectal Anastomosis for Ulcerative Colitis: A GETAID/GETAID Chirurgie Multicenter Retrospective Cohort of 343 Patients. Ann Surg 2017; 266: $1029-1034$

[607] Borjesson L, Lundstam U, Oresland T et al. The place for colectomy and ileorectal anastomosis: a valid surgical option for ulcerative colitis? Tech Coloproctol 2006; 10: 237-241; discussion 241

[608] da Luz Moreira A, Kiran RP, Lavery I. Clinical outcomes of ileorectal anastomosis for ulcerative colitis. Br J Surg 2010; 97: 65 -69

[609] Soravia C, O’Connor BI, Berk T et al. Functional outcome of conversion of ileorectal anastomosis to ileal pouch-anal anastomosis in patients with familial adenomatous polyposis and ulcerative colitis. Dis Colon Rectum 1999; 42: $903-908$

[610] Nessar G, Fazio VW, Tekkis P et al. Long-term outcome and quality of life after continent ileostomy. Dis Colon Rectum 2006; 49: 336 - 344

[611] Berndtsson I, Lindholm E, Ekman I. Thirty years of experience living with a continent ileostomy: bad restrooms-not my reservoir-decide my life. J Wound Ostomy Continence Nurs 2005; 32: 321 - 326; quiz 327-328

[612] Litle VR, Barbour S, Schrock TR et al. The continent ileostomy: longterm durability and patient satisfaction. J Gastrointest Surg 1999; 3: $625-632$
[613] Lian L, Fazio VW, Remzi FH et al. Outcomes for patients undergoing continent ileostomy after a failed ileal pouch-anal anastomosis. Dis Colon Rectum 2009; 52: 1409-1414; discussion 4414-4416

[614] Uzzan M, Kirchgesner J, Oubaya N et al. Risk of Rectal Neoplasia after Colectomy and Ileorectal Anastomosis for Ulcerative Colitis. J Crohns Colitis 2017; 11: $930-935$

[615] Kuiper T, Vlug MS, van den Broek FJ et al. The prevalence of dysplasia in the ileoanal pouch following restorative proctocolectomy for ulcerative colitis with associated dysplasia. Colorectal Dis 2012; 14: 469 473

[616] Maartense S, Dunker MS, Slors JF et al. Hand-assisted laparoscopic versus open restorative proctocolectomy with ileal pouch anal anastomosis: a randomized trial. Ann Surg 2004; 240: 984-991; discussion 991-992

[617] Tilney HS, Lovegrove RE, Heriot AG et al. Comparison of short-term outcomes of laparoscopic vs open approaches to ileal pouch surgery. Int J Colorectal Dis 2007; 22: 531 - 542

[618] Ahmed AliU, Keus F, Heikens JT et al. Open versus laparoscopic (assisted) ileo pouch anal anastomosis for ulcerative colitis and familial adenomatous polyposis. Cochrane Database Syst Rev 2009; 21: CD006267

[619] Bartels SA, Gardenbroek T], Ubbink DT et al. Systematic review and meta-analysis of laparoscopic versus open colectomy with end ileostomy for non-toxic colitis. Br J Surg 2013; 100: 726-733

[620] Wu XJ, He XS, Zhou XY et al. The role of laparoscopic surgery for ulcerative colitis: systematic review with meta-analysis. Int J Colorectal Dis 2010; 25: $949-957$

[621] Beyer-Berjot L, Maggiori L, Birnbaum D et al. A total laparoscopic approach reduces the infertility rate after ileal pouch-anal anastomosis: a 2-center study. Ann Surg 2013; 258: 275-282

[622] Bartels SA, Vlug MS, Henneman D et al. Less adhesiolysis and hernia repair during completion proctocolectomy after laparoscopic emergency colectomy for ulcerative colitis. Surg Endosc 2012; 26: 368 373

[623] Indar AA, Efron JE, Young-Fadok TM. Laparoscopic ileal pouch-anal anastomosis reduces abdominal and pelvic adhesions. Surg Endosc 2009; 23: 174-177

[624] Bartels SA, D’Hoore A, Cuesta MA et al. Significantly increased pregnancy rates after laparoscopic restorative proctocolectomy: a crosssectional study. Ann Surg 2012; 256: 1045 - 1048

[625] Benlice C, Stocchi L, Costedio M et al. Laparoscopic IPAA is not associated with decreased rates of incisional hernia and small-bowel obstruction when compared with open technique: long-term follow-up of a case-matched study. Dis Colon Rectum 2015; 58: 314-320

[626] Holubar SD, Larson DW, Dozois EJ et al. Minimally invasive subtotal colectomy and ileal pouch-anal anastomosis for fulminant ulcerative colitis: a reasonable approach? Dis Colon Rectum 2009; 52: 187-192

[627] Chung TP, Fleshman JW, Birnbaum EH et al. Laparoscopic vs. open total abdominal colectomy for severe colitis: impact on recovery and subsequent completion restorative proctectomy. Dis Colon Rectum 2009; 52: 4-10

[628] Telem DA, Vine AJ, Swain G et al. Laparoscopic subtotal colectomy for medically refractory ulcerative colitis: the time has come. Surg Endosc 2010; $24: 1616-1620$

[629] Geboes K, Colombel JF, Greenstein A et al. Indeterminate colitis: a review of the concept-what's in a name? Inflamm Bowel Dis 2008; 14 : $850-857$

[630] Pishori T, Dinnewitzer A, Zmora O et al. Outcome of patients with indeterminate colitis undergoing a double-stapled ileal pouch-anal anastomosis. Dis Colon Rectum 2004; 47: 717 - 721

[631] Delaney CP, Remzi FH, Gramlich T et al. Equivalent function, quality of life and pouch survival rates after ileal pouch-anal anastomosis for indeterminate and ulcerative colitis. Ann Surg 2002; 236: 43-48 
[632] Reese GE, Lovegrove RE, Tilney HS et al. The effect of Crohn's disease on outcomes after restorative proctocolectomy. Dis Colon Rectum 2007; 50: $239-250$

[633] Malaty HM, Fan X, Opekun AR et al. Rising incidence of inflammatory bowel disease among children: a 12-year study. J Pediatr Gastroenterol Nutr 2010; 50: $27-31$

[634] Svaninger G, Nordgren S, Oresland T et al. Incidence and characteristics of pouchitis in the Kock continent ileostomy and the pelvic pouch. Scand J Gastroenterol 1993; 28: 695-700

[635] Rauh SM, Schoetz DJ Jr, Roberts PL et al. Pouchitis-is it a wastebasket diagnosis? Dis Colon Rectum 1991; 34: 685-689

[636] Sandborn WJ, Tremaine WJ, Batts KP et al. Pouchitis after ileal pouchanal anastomosis: a Pouchitis Disease Activity Index. Mayo Clin Proc 1994; 69: 409-415

[637] Tiainen J, Matikainen M. Long-term clinical outcome and anemia after restorative proctocolectomy for ulcerative colitis. Scand J Gastroenterol 2000; 35: 1170-1173

[638] Meagher AP, Farouk R, Dozois RR et al. J ileal pouch-anal anastomosis for chronic ulcerative colitis: complications and long-term outcome in 1310 patients. $\mathrm{Br}$ J Surg 1998; 85: $800-803$

[639] Hurst RD, Chung TP, Rubin M et al. The implications of acute pouchitis on the long-term functional results after restorative proctocolectomy. Inflamm Bowel Dis 1998; 4: 280-284

[640] Tiainen J, Matikainen M, Aitola P et al. Histological and macroscopic changes in the pelvic pouch: long-term follow up after restorative proctocolectomy for ulcerative colitis (UC). Colorectal Dis 2001; 3: $28-32$

[641] Tiainen J, Matikainen M. Health-related quality of life after ileal Jpouch-anal anastomosis for ulcerative colitis: long-term results. Scand J Gastroenterol 1999; 34: 601-605

[642] Seidel SA, Peach SE, Newman M et al. Ileoanal pouch procedures: clinical outcomes and quality-of-life assessment. Am Surg 1999; 65: $40-46$

[643] Heuschen UA, Autschbach F, Allemeyer EH et al. Long-term follow-up after ileoanal pouch procedure: algorithm for diagnosis, classification, and management of pouchitis. Dis Colon Rectum 2001; 44: 487 - 499

[644] Stahlberg D, Gullberg K, Liljeqvist L et al. Pouchitis following pelvic pouch operation for ulcerative colitis. Incidence, cumulative risk, and risk factors. Dis Colon Rectum 1996; 39: 1012-1018

[645] Lohmuller JL, Pemberton JH, Dozois RR et al. Pouchitis and extraintestinal manifestations of inflammatory bowel disease after ileal pouch-anal anastomosis. Ann Surg 1990; 211: 622-627; discussion 627-629

[646] Nicholls RJ, Banerjee AK. Pouchitis: risk factors, etiology, and treatment. World J Surg 1998; 22: 347 - 351

[647] Penna C, Dozois R, Tremaine W et al. Pouchitis after ileal pouch-anal anastomosis for ulcerative colitis occurs with increased frequency in patients with associated primary sclerosing cholangitis. Gut 1996; 38 : 234- 239

[648] Shen B, Achkar JP, Lashner BA et al. Irritable pouch syndrome: a new category of diagnosis for symptomatic patients with ileal pouch-anal anastomosis. Am J Gastroenterol 2002; 97: 972 - 977

[649] Thoeni RF, Fell SC, Engelstad B et al. Ileoanal pouches: comparison of $\mathrm{CT}$, scintigraphy, and contrast enemas for diagnosing postsurgical complications. Am J Roentgenol 1990; 154: 73-78

[650] Libicher M, Scharf ], Wunsch A et al. MRI of pouch-related fistulas in ulcerative colitis after restorative proctocolectomy. J Comput Assist Tomogr 1998; 22: 664-668

[651] Hrung JM, Levine MS, Rombeau JL et al. Total proctocolectomy and ileoanal pouch: the role of contrast studies for evaluating postoperative leaks. Abdom Imaging 1998; 23: 375- 379
[652] Solomon M], McLeod RS, O'Connor Bl et al. Assessment of peripouch inflammation after ileoanal anastomosis using endoluminal ultrasonography. Dis Colon Rectum 1995; 38: $182-187$

[653] Segal JP, Ding NS, Worley G et al. Systematic review with meta-analysis: the management of chronic refractory pouchitis with an evidencebased treatment algorithm. Aliment Pharmacol Ther 2017; 45: 581 592

[654] Sandborn W, McLeod R, Jewell D. Pharmacotherapy for inducing and maintaining remission in pouchitis. Cochrane Database Syst Rev 2000; 2: CD001176

[655] Sandborn WJ, McLeod R, Jewell DP. Medical therapy for induction and maintenance of remission in pouchitis: a systematic review. Inflamm Bowel Dis 1999; 5: 33-39

[656] Singh S, Stroud AM, Holubar SD et al. Treatment and prevention of pouchitis after ileal pouch-anal anastomosis for chronic ulcerative colitis. Cochrane Database Syst Rev 2015; 23: CD001176

[657] Mimura T, Rizzello F, Helwig U et al. Four-week open-label trial of metronidazole and ciprofloxacin for the treatment of recurrent or refractory pouchitis. Aliment Pharmacol Ther 2002; 16: 909-917

[658] Madden MV, McIntyre AS, Nicholls RJ. Double-blind crossover trial of metronidazole versus placebo in chronic unremitting pouchitis. Dig Dis Sci 1994; 39: $1193-1196$

[659] Shen B, Achkar JP, Lashner BA et al. A randomized clinical trial of ciprofloxacin and metronidazole to treat acute pouchitis. Inflamm Bowel Dis 2001; 7: $301-305$

[660] Hurst RD, Molinari M, Chung TP et al. Prospective study of the incidence, timing and treatment of pouchitis in 104 consecutive patients after restorative proctocolectomy. Arch Surg 1996; 131: 497 - 500; discussion 501-502

[661] Shen B, Fazio VW, Remzi FH et al. Combined ciprofloxacin and tinidazole therapy in the treatment of chronic refractory pouchitis. Dis Colon Rectum 2007; 50: 498-508

[662] Gionchetti P, Rizzello F, Venturi A et al. Antibiotic combination therapy in patients with chronic, treatment-resistant pouchitis. Aliment Pharmacol Ther 1999; 13: 713-718

[663] Nygaard K, Bergan T, Bjorneklett A et al. Topical metronidazole treatment in pouchitis. Scand J Gastroenterol 1994; 29: 462-467

[664] Abdelrazeq AS, Kelly SM, Lund JN et al. Rifaximin-ciprofloxacin combination therapy is effective in chronic active refractory pouchitis. Colorectal Dis 2005; 7: $182-186$

[665] Gionchetti P, Rizzello F, Poggioli G et al. Oral budesonide in the treatment of chronic refractory pouchitis. Aliment Pharmacol Ther 2007; 25: $1231-1236$

[666] Sambuelli A, Boerr L, Negreira S et al. Budesonide enema in pouchitisa double-blind, double-dummy, controlled trial. Aliment Pharmacol Ther 2002; 16: $27-34$

[667] Herfarth HH, Long MD, Isaacs KL. Use of Biologics in Pouchitis: A Systematic Review. J Clin Gastroenterol 2015; 49: 647 -654

[668] Kelly OB, Rosenberg M, Tyler AD et al. Infliximab to Treat Refractory Inflammation After Pelvic Pouch Surgery for Ulcerative Colitis. J Crohns Colitis 2016; 10: 410-417

[669] Li Y, Lopez R, Queener E et al. Adalimumab therapy in Crohn's disease of the ileal pouch. Inflamm Bowel Dis 2012; 18: 2232-2239

[670] Barreiro-de Acosta M, Garcia-Bosch O, Gordillo J et al. Efficacy of adalimumab rescue therapy in patients with chronic refractory pouchitis previously treated with infliximab: a case series. Eur J Gastroenterol Hepatol 2012; 24: $756-758$

[671] Mir F, Yousef MH, Partyka EK et al. Successful treatment of chronic refractory pouchitis with vedolizumab. Int J Colorectal Dis 2017; 32: $1517-1518$ 
[672] Tran-Minh ML, Allez M, Gornet JM. Successful Treatment With Ustekinumab for Chronic Refractory Pouchitis. J Crohns Colitis 2017; 11: 1156

[673] Winter TA, Dalton HR, Merrett MN et al. Cyclosporin A retention enemas in refractory distal ulcerative colitis and 'pouchitis'. Scand J Gastroenterol 1993; 28: $701-704$

[674] Fang S, Kraft CS, Dhere T et al. Successful treatment of chronic Pouchitis utilizing fecal microbiota transplantation (FMT): a case report. Int J Colorectal Dis 2016; 31: 1093 - 1094

[675] Schmid M, Frick JS, Malek N et al. Successful treatment of pouchitis with Vedolizumab, but not fecal microbiota transfer (FMT), after proctocolectomy in ulcerative colitis. Int J Colorectal Dis 2017; 32: 597 598

[676] Miner P, Wedel M, Bane B et al. An enema formulation of alicaforsen, an antisense inhibitor of intercellular adhesion molecule-1, in the treatment of chronic, unremitting pouchitis. Aliment Pharmacol Ther 2004; 19: 281 - 286

[677] Shen B, Remzi FH, Lopez AR et al. Rifaximin for maintenance therapy in antibiotic-dependent pouchitis. BMC Gastroenterol 2008; 8: 26

[678] Greuter T, Biedermann L, Rogler G et al. Alicaforsen, an antisense inhibitor of ICAM-1, as treatment for chronic refractory pouchitis after proctocolectomy: A case series. United European Gastroenterol J 2016; 4: $97-104$

[679] Shen B, Remzi FH, Lavery IC et al. Administration of adalimumab in the treatment of Crohn's disease of the ileal pouch. Aliment Pharmacol Ther 2009; 29: 519-526

[680] Holubar SD, Cima RR, Sandborn WJ et al. Treatment and prevention of pouchitis after ileal pouch-anal anastomosis for chronic ulcerative colitis. Cochrane Database Syst Rev 2010; 16: CD001176

[681] Mimura T, Rizzello F, Helwig U et al. Once daily high dose probiotic therapy (VSL\#3) for maintaining remission in recurrent or refractory pouchitis. Gut 2004; 53: $108-114$

[682] Gionchetti P, Rizzello F, Venturi A et al. Oral bacteriotherapy as maintenance treatment in patients with chronic pouchitis: a double-blind placebo-controlled trial. Gastroenterology 2000; 119: 305-309

[683] Zhu H, Wu XR, Queener E et al. Clinical value of surveillance pouchoscopy in asymptomatic ileal pouch patients with underlying inflammatory bowel disease. Surg Endosc 2013; 27: 4325-4332

[684] Gullberg K, Lindforss U, Zetterquist $\mathrm{H}$ et al. Cancer risk assessment in long-standing pouchitis. DNA aberrations are rare in transformed neoplastic pelvic pouch mucosa. Int J Colorectal Dis 2002; 17: 92 - 97

[685] Hashimoto T, Itabashi M, Ogawa S et al. Treatment strategy for preventing pouchitis as a postoperative complication of ulcerative colitis: the significance of the management of cuffitis. Surg Today 2014; 44: $1730-1734$

[686] Shen B, Lashner BA, Bennett AE et al. Treatment of rectal cuff inflammation (cuffitis) in patients with ulcerative colitis following restorative proctocolectomy and ileal pouch-anal anastomosis. Am J Gastroenterol 2004; 99: 1527-1531

[687] Shen B, Sanmiguel C, Bennett AE et al. Irritable pouch syndrome is characterized by visceral hypersensitivity. Inflamm Bowel Dis 2011; 17: 994-1002

[688] Hou JK, Abraham B, El-Serag H. Dietary intake and risk of developing inflammatory bowel disease: a systematic review of the literature. Am J Gastroenterol 2011; 106: 563-573

[689] Kruis W, Fric P, Pokrotnieks ] et al. Maintaining remission of ulcerative colitis with the probiotic Escherichia coli Nissle 1917 is as effective as with standard mesalazine. Gut 2004; 53: 1617-1623

[690] Yoshimatsu Y, Yamada A, Furukawa R et al. Effectiveness of probiotic therapy for the prevention of relapse in patients with inactive ulcerative colitis. World J Gastroenterol 2015; 21: 5985 - 5994
[691] Gionchetti P, Rizzello F, Morselli C et al. High-dose probiotics for the treatment of active pouchitis. Dis Colon Rectum 2007; 50: 20752082; discussion 2082-2084

[692] Klement E, Reif S. Breastfeeding and risk of inflammatory bowel disease. Am J Clin Nutr 2005; 82: 486

[693] Halmos EP, Muir JG, Barrett JS et al. Diarrhoea during enteral nutrition is predicted by the poorly absorbed short-chain carbohydrate (FODMAP) content of the formula. Aliment Pharmacol Ther 2010; 32: 925 933

[694] Gearry RB, Richardson AK, Frampton CM et al. Population-based cases control study of inflammatory bowel disease risk factors. J Gastroenterol Hepatol 2010; 25: 325-333

[695] Zocco MA, dal Verme LZ, Cremonini F et al. Efficacy of Lactobacillus GG in maintaining remission of ulcerative colitis. Aliment Pharmacol Ther 2006; 23: $1567-1574$

[696] Kappelman MD, Bousvaros A. Nutritional concerns in pediatric inflammatory bowel disease patients. Mol Nutr Food Res 2008; 52: 867 - 874

[697] Nguyen GC, Munsell M, Harris ML. Nationwide prevalence and prognostic significance of clinically diagnosable protein-calorie malnutrition in hospitalized inflammatory bowel disease patients. Inflamm Bowel Dis 2008; 14: 1105-1111

[698] Massironi S, Rossi RE, Cavalcoli FA et al. Nutritional deficiencies in inflammatory bowel disease: therapeutic approaches. Clin Nutr 2013; 32: $904-910$

[699] Han YM, Yoon H, Lim S et al. Risk Factors for Vitamin D, Zinc, and Selenium Deficiencies in Korean Patients with Inflammatory Bowel Disease. Gut Liver 2017; 11: 363-369

[700] Oikonomou IK, Fazio VW, Remzi FH et al. Risk factors for anemia in patients with ileal pouch-anal anastomosis. Dis Colon Rectum 2007; 50: $69-74$

[701] Pastrana RJ, Torres EA, Arroyo JM et al. Iron-deficiency anemia as presentation of pouchitis. J Clin Gastroenterol 2007; 41: 41 - 44

[702] Prince AC, Myers CE, Joyce T et al. Fermentable Carbohydrate Restriction (Low FODMAP Diet) in Clinical Practice Improves Functional Gastrointestinal Symptoms in Patients with Inflammatory Bowel Disease. Inflamm Bowel Dis 2016; 22: 1129-1136

[703] M'Koma AE. Follow-up results of hematology data before and after restorative proctocolectomy. Clinical outcome. Dis Colon Rectum 1994; 37: $932-937$

[704] Bischoff SC, Koletzko B, Lochs $\mathrm{H}$ et al. Klinische Ernährung in der Gastroenterologie (Teil 4) - Chronisch-entzündliche Darmerkrankungen. Aktuel Ernahrungsmed 2014; 39: e72-e98

[705] Turner D, Zlotkin SH, Shah PS et al. Omega 3 fatty acids (fish oil) for maintenance of remission in Crohn's disease. Cochrane Database Syst Rev 2009; 21: CD006320

[706] Turner D, Shah PS, Steinhart AH et al. Maintenance of remission in inflammatory bowel disease using omega-3 fatty acids (fish oil): a systematic review and meta-analyses. Inflamm Bowel Dis 2011; 17: 336 345

[707] Klement E, Cohen RV, Boxman J et al. Breastfeeding and risk of inflammatory bowel disease: a systematic review with meta-analysis. Am J Clin Nutr 2004; 80: 1342-1352

[708] Akobeng AK, Elawad M, Gordon M. Glutamine for induction of remission in Crohn's disease. Cochrane Database Syst Rev 2016; 2: CD007348

[709] Lewis JD, Abreu MT. Diet as a Trigger or Therapy for Inflammatory Bowel Diseases. Gastroenterology 2017; 152: 398-414 e6

[710] Lochs H, Dejong C, Hammarqvist F et al. ESPEN Guidelines on Enteral Nutrition: Gastroenterology. Clin Nutr 2006; 25: 260-274

[711] Schulz RJ, Bischoff SC, Koletzko B et al. Gastroenterology - Guidelines on Parenteral Nutrition, Chapter 15. Ger Med Sci 2009; 7: Doc13 
[712] Forbes A, Escher J, Hebuterne X et al. ESPEN guideline: Clinical nutrition in inflammatory bowel disease. Clin Nutr 2017; 36: 321 - 347

[713] Song HK, Buzby GP. Nutritional support for Crohn's disease. Surg Clin North Am 2001; 81: $103-115$, viii

[714] Nguyen DL, Parekh NK, Jamal MM. National Trends and Outcomes of Inflammatory Bowel Disease Patients Requiring in-Hospital Total Parenteral Nutrition Support. Gastroenterology 2013; 144: S749-S749

[715] Bischoff SC, Fleig W. Ulcerative colitis. Nutrition. Z Gastroenterol 2004; 42: $998-1002$

[716] Stein J. Leitlinien Morbus Crohn. Z Gastroenterol 2003; 41: 62 -68

[717] Lee D, Albenberg L, Compher C et al. Diet in the pathogenesis and treatment of inflammatory bowel diseases. Gastroenterology 2015; 148: $1087-1106$

[718] Martin J, Radeke HH, Dignass A et al. Current evaluation and management of anemia in patients with inflammatory bowel disease. Expert Rev Gastroenterol Hepatol 2017; 11: 19-32

[719] Evstatiev R, Marteau P, Iqbal T et al. FERGIcor, a randomized controlled trial on ferric carboxymaltose for iron deficiency anemia in inflammatory bowel disease. Gastroenterology 2011; 141: 846-853 e1-2

[720] Wolman SL, Anderson GH, Marliss EB et al. Zinc in total parenteral nutrition: requirements and metabolic effects. Gastroenterology 1979; 76: $458-467$

[721] Jeejeebhoy K. Zinc: an essential trace element for parenteral nutrition. Gastroenterology 2009; 137: S7 - S12

[722] Schölmerich J, Freudemann A, Köttgen E et al. Bioavailability of zinc from zinc-histidine complexes. I. Comparison with zinc sulfate in healthy men. Am J Clin Nutr 1987; 45: 1480-1486

[723] Rossi RE, Whyand T, Murray CD et al. The role of dietary supplements in inflammatory bowel disease: a systematic review. Eur J Gastroenterol Hepatol 2016; 28: 1357 - 1364

[724] Fessler TA. Trace elements in parenteral nutrition: a practical guide for dosage and monitoring for adult patients. Nutr Clin Pract 2013; 28 : $722-729$

[725] Kruis W, Phuong Nguyen G. Iron Deficiency, Zinc, Magnesium, Vitamin Deficiencies in Crohn's Disease: Substitute or Not? Dig Dis 2016; 34: $105-111$

[726] Hlavaty T, Krajcovicova A, Payer J. Vitamin D therapy in inflammatory bowel diseases: who, in what form, and how much? J Crohns Colitis 2015; 9: 198-209

[727] Raftery T, O’Sullivan M. Optimal vitamin D levels in Crohn's disease: a review. Proc Nutr Soc 2015; 74: 56-66

[728] Lee JA, Hwang JS, Hwang IT et al. Low vitamin D levels are associated with both iron deficiency and anemia in children and adolescents. Pediatr Hematol Oncol 2015; 32: 99-108

[729] Laake KO, Bjorneklett A, Aamodt G et al. Outcome of four weeks' intervention with probiotics on symptoms and endoscopic appearance after surgical reconstruction with a J-configurated ileal-pouch-analanastomosis in ulcerative colitis. Scand J Gastroenterol 2005; 40: 43 51

[730] Ng SC, Plamondon S, Kamm MA et al. Immunosuppressive effects via human intestinal dendritic cells of probiotic bacteria and steroids in the treatment of acute ulcerative colitis. Inflamm Bowel Dis 2010; 16: $1286-1298$

[731] Laake KO, Line PD, Aabakken L et al. Assessment of Mucosal Inflammation and Circulation in Response to Probiotics in Patients Operated with Ileal Pouch Anal Anastomosis for Ulcerative Colitis. Scand J Gastroenterol 2003; 38: 409-414

[732] Higgens CS, Keighley MR, Allan RN. Impact of preoperative weight loss and body composition changes on postoperative outcome in surgery for inflammatory bowel disease. Gut 1984; 25: 732-736
[733] Lindor KD, Fleming CR, Ilstrup DM. Preoperative nutritional status and other factors that influence surgical outcome in patients with Crohn's disease. Mayo Clin Proc 1985; 60: 393-396

[734] Rombeau JL, Barot LR, Williamson CE et al. Preoperative total parenteral nutrition and surgical outcome in patients with inflammatory bowel disease. Am J Surg 1982; 143: 139-143

[735] Schwartz E. Perioperative Parenteral Nutrition in Adults With Inflammatory Bowel Disease: A Review of the Literature. Nutr Clin Pract 2016; 31: 159-170

[736] Weimann A, Braga M, Harsanyi L et al. ESPEN Guidelines on Enteral Nutrition: Surgery including organ transplantation. Clin Nutr 2006; 25 : $224-244$

[737] Sorensen J, Kondrup ], Prokopowicz J et al. EuroOOPS: an international, multicentre study to implement nutritional risk screening and evaluate clinical outcome. Clin Nutr 2008; 27: 340 -349

[738] Weimann A, Breitenstein S, Breuer JP et al. Clinical nutrition in surgery. Guidelines of the German Society for Nutritional Medicine. Chirurg 2014; 85: $320-326$

[739] Stein J. Kurzdarmsyndrom - Chronisches Darmversagen. In: E.D. S, ed Entzündliche Darmerkrankungen. Stuttgart: Schattauer; 2015: 361 386

[740] M'Koma AE, Wise PE, Schwartz DA et al. Prevalence and outcome of anemia after restorative proctocolectomy: a clinical literature review. Dis Colon Rectum 2009; 52: 726-739

[741] Stein J, Connor S, Virgin G et al. Anemia and iron deficiency in gastrointestinal and liver conditions. World J Gastroenterol 2016; 22: 7908 7925

[742] M'Koma AE. Serum biochemical evaluation of patients with functional pouches ten to 20 years after restorative proctocolectomy. Int J Colorectal Dis 2006; 21: $711-720$

[743] Khanna R, Wu X, Shen B. Low levels of vitamin D are common in patients with ileal pouches irrespective of pouch inflammation. J Crohns Colitis 2013; 7: $525-533$

[744] Kuisma J, Nuutinen $\mathrm{H}$, Luukkonen P et al. Long term metabolic consequences of ileal pouch-anal anastomosis for ulcerative colitis. Am J Gastroenterol 2001; 96: 3110-3116

[745] Wieland LS, Manheimer E, Berman BM. Development and classification of an operational definition of complementary and alternative medicine for the Cochrane collaboration. Altern Ther Health Med 2011; 17 : $50-59$

[746] Barnes PM, Bloom B, Nahin RL. Complementary and alternative medicine use among adults and children: United States, 2007. Natl Health Stat Report 2008; 10: $1-23$

[747] Bensoussan M, Jovenin N, Garcia B et al. Complementary and alternative medicine use by patients with inflammatory bowel disease: results from a postal survey. Gastroenterol Clin Biol 2006; 30: 14-23

[748] Burgmann T, Rawsthorne P, Bernstein CN. Predictors of alternative and complementary medicine use in inflammatory bowel disease: do measures of conventional health care utilization relate to use? Am J Gastroenterol 2004; 99: 889-893

[749] Hilsden RJ, Meddings JB, Verhoef MJ. Complementary and alternative medicine use by patients with inflammatory bowel disease: An Internet survey. Can J Gastroenterol 1999; 13: 327-332

[750] Hilsden RJ, Verhoef MJ, Rasmussen $\mathrm{H}$ et al. Use of complementary and alternative medicine by patients with inflammatory bowel disease. Inflamm Bowel Dis 2011; 17: 655-662

[751] Kong SC, Hurlstone DP, Pocock CY et al. The Incidence of self-prescribed oral complementary and alternative medicine use by patients with gastrointestinal diseases. J Clin Gastroenterol 2005; 39: 138-141 
[752] Langhorst ], Anthonisen IB, Steder-Neukamm U et al. Amount of systemic steroid medication is a strong predictor for the use of complementary and alternative medicine in patients with inflammatory bowel disease: results from a German national survey. Inflamm Bowel Dis 2005; 11: $287-295$

[753] Langhorst J, Wulfert H, Lauche R et al. Systematic review of complementary and alternative medicine treatments in inflammatory bowel diseases. J Crohns Colitis 2015; 9: 86-106

[754] Elsenbruch S, Langhorst ], Popkirowa K et al. Effects of mind-body therapy on quality of life and neuroendocrine and cellular immune functions in patients with ulcerative colitis. Psychother Psychosom 2005; 74: 277-287

[755] Langhorst ], Mueller T, Luedtke R et al. Effects of a comprehensive lifestyle modification program on quality-of-life in patients with ulcerative colitis: a twelve-month follow-up. Scand J Gastroenterol 2007; 42: $734-745$

[756] Berrill JW, Sadlier M, Hood K et al. Mindfulness-based therapy for inflammatory bowel disease patients with functional abdominal symptoms or high perceived stress levels. J Crohns Colitis 2014; 8: 945-955

[757] Jedel S, Hoffman A, Merriman P et al. A randomized controlled trial of mindfulness-based stress reduction to prevent flare-up in patients with inactive ulcerative colitis. Digestion 2014; 89: 142 - 155

[758] Mizrahi MC, Reicher-Atir R, Levy S et al. Effects of guided imagery with relaxation training on anxiety and quality of life among patients with inflammatory bowel disease. Psychology and Health 2012; 27 : 1463 1479

[759] Shaw L, Ehrlich A. Relaxation training as a treatment for chronic pain caused by ulcerative colitis. Pain 1987; 29: $287-293$

[760] Gerbarg PL, Jacob VE, Stevens L et al. The Effect of Breathing, Movement, and Meditation on Psychological and Physical Symptoms and Inflammatory Biomarkers in Inflammatory Bowel Disease: A Randomized Controlled Trial. Inflammatory Bowel Diseases 2015; 21: 2886 2896

[761] Klare P, Nigg J, Nold J et al. The impact of a ten-week physical exercise program on health-related quality of life in patients with inflammatory bowel disease: A prospective randomized controlled trial. Digestion 2015; 91: 239-247

[762] Packer N, Hoffman-Goetz L, Ward G. Does physical activity affect quality of life, disease symptoms and immune measures in patients with inflammatory bowel disease? A systematic review. Journal of Sports Medicine and Physical Fitness 2010; 50: 1 - 18

[763] Sharma P, Poojary G, Velez DM et al. Effect of Yoga-Based Intervention in Patients with Inflammatory Bowel Disease. Int J Yoga Therap 2015; 25: $101-112$

[764] Cramer H, Schafer M, Schols M et al. Randomised clinical trial: yoga vs written self-care advice for ulcerative colitis. Aliment Pharmacol Ther 2017; 45: 1379-1389

[765] Schneider A, Streitberger K, Joos S. Acupuncture treatment in gastrointestinal diseases: A systematic review. World J Gastroenterol 2007; 13: $3417-3424$

[766] Joos S, Wildau N, Kohnen R et al. Acupuncture and moxibustion in the treatment of ulcerative colitis: a randomized controlled study. Scand J Gastroenterol 2006; 41: 1056-1063

[767] Ji J, Lu Y, Liu H et al. Acupuncture and moxibustion for inflammatory bowel diseases: a systematic review and meta-analysis of randomized controlled trials. Evid Based Complement Alternat Med 2013; 2013: 158352

[768] Lee DH, Kim JI, Lee MS et al. Moxibustion for ulcerative colitis: a systematic review and meta-analysis. BMC Gastroenterol 2010; 10: 36

[769] Fernandez-Banares F, Hinojosa J, Sanchez-Lombrana JL et al. Randomized clinical trial of Plantago ovata seeds (dietary fiber) as compared with mesalamine in maintaining remission in ulcerative colitis. Spanish
Group for the Study of Crohn's Disease and Ulcerative Colitis (GETECCU). Am J Gastroenterol 1999; 94: 427-433

[770] Hanai H, lida T, Takeuchi K et al. Curcumin Maintenance Therapy for Ulcerative Colitis: Randomized, Multicenter, Double-Blind, PlaceboControlled Trial. Clinical Gastroenterology and Hepatology 2006; 4: $1502-1506$

[771] Singla V, Pratap Mouli V, Garg SK et al. Induction with NCB-02 (curcumin) enema for mild-to-moderate distal ulcerative colitis - a randomized, placebo-controlled, pilot study. J Crohns Colitis 2014; 8: 208 214

[772] Lang A, Salomon N, Wu JCY et al. Curcumin in Combination With Mesalamine Induces Remission in Patients With Mild-to-Moderate Ulcerative Colitis in a Randomized Controlled Trial. Clinical Gastroenterology and Hepatology 2015; 13: 1444 -1449.e1

[773] Langhorst J, Varnhagen I, Schneider SB et al. Randomised clinical trial: a herbal preparation of myrrh, chamomile and coffee charcoal compared with mesalazine in maintaining remission in ulcerative colitis a double-blind, double-dummy study. Aliment Pharmacol Ther 2013; 38: $490-500$

[774] Albrecht U, Muller V, Schneider B et al. Efficacy and safety of a herbal medicinal product containing myrrh, chamomile and coffee charcoal for the treatment of gastrointestinal disorders: a non-interventional study. BMJ Open Gastroenterol 2014; 1: e000015

[775] Langhorst], Lauche R, Koch AK. Myrrhe, Kamille und Kaffeekohle in der Therapie von Patienten mit Colitis ulcerosa. Eine retrospektive Kohortenstudie mit 5-Jahres-Follow-up. Zeitschrift für Phytotherapie 2016; 37: $249-253$

[776] Kamali M, Tavakoli H, Khodadoost M et al. Efficacy of the Punica granatum peels aqueous extract for symptom management in ulcerative colitis patients. A randomized, placebo-controlled, clinical trial. Complement Ther Clin Pract 2015; 21: 141 - 146

[777] Gupta I, Parihar A, Malhotra P et al. Effects of gum resin of Boswellia serrata in patients with chronic colitis. Planta Med 2001; 67: 391 - 395

[778] Gupta I, Parihar A, Malhotra P et al. Effects of Boswellia serrata gum resin in patients with ulcerative colitis. Eur J Med Res 1997; 2: 37 - 43

[779] Tang T, Targan SR, Li ZS et al. Randomised clinical trial: herbal extract HMPL-004 in active ulcerative colitis - a double-blind comparison with sustained release mesalazine. Aliment Pharmacol Ther 2011; 33: 194 202

[780] Sandborn W], Targan SR, Byers VS et al. Andrographis paniculata extract (HMPL-004) for active ulcerative colitis. Am J Gastroenterol 2013; 108: $90-98$

[781] Ben-Arye E, Goldin E, Wengrower D et al. Wheat grass juice in the treatment of active distal ulcerative colitis: a randomized double-blind placebo-controlled trial. Scand J Gastroenterol 2002; 37: 444-449

[782] Greenfield SM, Green AT, Teare JP et al. A randomized controlled study of evening primrose oil and fish oil in ulcerative colitis. Aliment Pharmacol Ther 1993; 7: 159-166

[783] Langmead L, Feakins RM, Goldthorpe S et al. Randomized, doubleblind, placebo-controlled trial of oral aloe vera gel for active ulcerative colitis. Aliment Pharmacol Ther 2004; 19: 739-747

[784] Rastegarpanah M, Malekzadeh R, Vahedi $\mathrm{H}$ et al. A randomized, double blinded, placebo-controlled clinical trial of silymarin in ulcerative colitis. Chin J Integr Med 2015; 21: $902-906$

[785] Tong ZQ, Yang B, Chen BY et al. A multi-center, randomized, singleblind, controlled clinical study on the efficacy of composite sophora colon-soluble capsules in treating ulcerative colitis. Chin J Integr Med 2010; 16: 486-492

[786] Biedermann L, Mwinyi J, Scharl M et al. Bilberry ingestion improves disease activity in mild to moderate ulcerative colitis - an open pilot study. J Crohns Colitis 2013; 7: 271 -279 
[787] Pullan RD, Rhodes J, Ganesh S et al. Transdermal nicotine for active ulcerative colitis. N Engl ] Med 1994; 330: 811 -815

[788] Sandborn W], Tremaine W], Offord KP et al. Transdermal nicotine for mildly to moderately active ulcerative colitis. A randomized, doubleblind, placebo-controlled trial. Ann Intern Med 1997; 126: 364-371

[789] Thomas GA, Rhodes J, Mani V et al. Transdermal nicotine as maintenance therapy for ulcerative colitis. N Engl J Med 1995; 332: 988 - 992

[790] Thomas GA, Rhodes J, Ragunath K et al. Transdermal nicotine compared with oral prednisolone therapy for active ulcerative colitis. Eur ] Gastroenterol Hepatol 1996; 8: $769-776$

[791] Pagoldh M, Hultgren E, Arnell P et al. Hyperbaric oxygen therapy does not improve the effects of standardized treatment in a severe attack of ulcerative colitis: A prospective randomized study. Scand J Gastroenterol 2013; 48: 1033 - 1040
[792] Garg SK, Croft AM, Bager P. Helminth therapy (worms) for induction of remission in inflammatory bowel disease. Cochrane Database Syst Rev 2014; 20: CD009400

[793] Summers RW, Elliott DE, Urban JF Jr et al. Trichuris suis therapy for active ulcerative colitis: a randomized controlled trial. Gastroenterology 2005; 128: $825-832$

[794] Scholmerich J, Fellermann K, Seibold FW et al. A Randomised, Doubleblind, Placebo-controlled Trial of Trichuris suis ova in Active Crohn's Disease. J Crohns Colitis 2017; 11: 390 -399 